Prepared for the U.S. Department of Energy

under Contract DE-AC05-76RL01830

\title{
Development of Models to Simulate Tracer Tests for Characterization of Enhanced Geothermal Systems
}
MD Williams
TB Watson
PW Reimus
DL Newell
VR Vermeul
KB Leecaster
PE Rose
EM Brauser
CA Dean

May 2013

\section{Pacific Northwest}

NATIONAL LABORATORY

Proudly Operated by Battelle Since 1965 


\title{
Development of Models to Simulate Tracer Tests for Characterization of Enhanced Geothermal Systems
}

\author{
MD Williams \\ PW Reimus ${ }^{1}$ \\ VR Vermeul \\ PE Rose ${ }^{2}$ \\ CA Dean ${ }^{1}$
}

\author{
TB Watson ${ }^{3}$ \\ DL Newell ${ }^{1}$ \\ KB Leecaster ${ }^{2}$ \\ EM Brauser ${ }^{2}$
}

May 2013

Prepared for

the U.S. Department of Energy

under Contract DE-AC05-76RL01830

Pacific Northwest National Laboratory

Richland, Washington 99352

\footnotetext{
${ }^{1}$ Los Alamos National Laboratory, Los Alamos, New Mexico.

${ }^{2}$ Energy and Geoscience Institute, University of Utah, Salt Lake City, Utah.

${ }^{3}$ Brookhaven National Laboratory, Upton, New York.
} 


\section{Abstract}

A comprehensive 2006 Massachusetts Institute of Technology (MIT) report found that electrical power derived from engineered (or enhanced) geothermal systems (EGSs) "represents a large, indigenous resource that could provide base-load electric power and heat that can have a major impact on the United States, while incurring minimal environmental impacts." EGS resources differ from high-grade hydrothermal resources in that they lack sufficient temperature distribution, permeability/porosity, fluid saturation, or recharge of reservoir fluids. Therefore, quantitative characterization of temperature distributions and the surface area available for heat transfer in EGS is necessary for the design and commercial development of the geothermal energy of a potential EGS site. The goal of this project is to provide integrated tracer and tracer interpretation tools to facilitate this characterization. This project was initially focused on tracer development with the application of perfluorinated tracer (PFT) compounds, non-reactive tracers used in numerous applications from atmospheric transport to underground leak detection, to geothermal systems, and evaluation of encapsulated PFTs that would release tracers at targeted reservoir temperatures. After the 2011 midyear project review and subsequent discussions with the U.S. Department of Energy Geothermal Technology Program, emphasis was shifted to interpretive tool development, testing, and validation. Subsurface modeling capabilities are an important component of this project for both the design of suitable tracers and the interpretation of data from in situ tracer tests, be they single- or multi-well tests.

This project was a collaborative effort between Brookhaven National Laboratory (BNL), Los Alamos National Laboratory (LANL), Pacific Northwest National Laboratory (PNNL), and the Energy and Geoscience Institute (EGI) at the University of Utah. The project covered a wide array of activities including bench-scale testing of tracers, numerical modeling, interpretation of existing tracer tests, and design and conducting field tracer tests at a demonstration EGS site. The initial PFT development was led by BNL along with high temperature column studies by LANL. Various numerical models described in this study were applied by PNNL and LANL to bench-scale batch and column testing results of conservative and reactive tracers with different mineralogy, temperature regimes, and flow rates. These models were also used to 1) analyze an existing field reactive and conservative tracer test data set at a naturally fractured geothermal site at Soda Lake, Nevada, and 2) design the reactive and conservative tracer tests for an EGS demonstration site near Newberry Crater, Oregon, that was led by Alta Rock Energy, Inc. PNNL and EGI led tracer testing operations during this EGS demonstration that was conducted from October to December 2012. The numerical models developed under this project for the Newberry Crater EGS test will provide a useful tool for interpreting tracer test response during the planned flowback operations planned in the spring of 2013. The purpose of this report is to describe the results of the tracer and model development for simulating and conducting tracer tests for characterizing EGS parameters. 


\section{Acronyms and Abbreviations}

\begin{tabular}{|c|c|}
\hline${ }^{\circ} \mathrm{C}$ & degree(s) Celsius \\
\hline $1 \mathrm{D}$ & one-dimensional \\
\hline $2 \mathrm{D}$ & two-dimensional \\
\hline $3 \mathrm{D}$ & three-dimensional \\
\hline $1.5 \mathrm{NS}$ & 1,5 naphthalene disulfonate \\
\hline 1,6-NDS & 1,6-naphthalene disulfonate \\
\hline $\mathrm{Ar}$ & argon \\
\hline BNL & Brookhaven National Laboratory \\
\hline $\mathrm{Ca}$ & calcium \\
\hline $\mathrm{CaCO}_{3}$ & calcite \\
\hline CEC & cation-exchange capacity \\
\hline $\mathrm{cm}$ & centimeter(s) \\
\hline $\mathrm{cm}^{2}$ & square centimeter(s) \\
\hline $\mathrm{cm}^{3}$ & cubic centimeter(s) \\
\hline Cs & cesium \\
\hline $\mathrm{d}$ & $\operatorname{day}(\mathrm{s})$ \\
\hline DOE & U.S. Department of Energy \\
\hline EGI & Energy and Geosciences Institute \\
\hline EGS & engineered (or enhanced) geothermal system \\
\hline $\mathrm{eq} / \mathrm{kg}$ & equivalents per kilogram \\
\hline $\mathrm{g}$ & $\operatorname{gram}(\mathrm{s})$ \\
\hline gal & gallon(s) \\
\hline gpm & gallon(s) per minute \\
\hline GTP & Geothermal Technology Program \\
\hline $\mathrm{H}_{2}$ & hydrogen \\
\hline $\mathrm{hr}$ & hour(s) \\
\hline $\mathrm{J}$ & joule(s) \\
\hline $\mathrm{K}$ & degree(s) Kelvin; or potassium \\
\hline kcal & kilocalories \\
\hline $\mathrm{K}_{d}$ & distribution coefficient \\
\hline $\mathrm{kg}$ & kilogram(s) \\
\hline $\mathrm{km}$ & kilometer(s) \\
\hline LANL & Los Alamos National Laboratory \\
\hline $\mathrm{Li}$ & lithium \\
\hline$\mu \mathrm{m}$ & micron(s) \\
\hline $\mathrm{m}$ & meter(s) \\
\hline
\end{tabular}




\begin{tabular}{ll}
$\mathrm{M}$ & Molar \\
$\mathrm{m}^{2}$ & square meter(s) \\
$\mathrm{meq} / \mathrm{kg}$ & milliequivalents per kilogram \\
$\mathrm{mg}$ & milligram(s) \\
$\mathrm{Mg}$ & magnesium \\
$\mathrm{MINC}$ & multiple interacting continua \\
$\mathrm{ml}$ & milliliter(s) \\
$\mathrm{mM}$ & millimolar \\
$\mathrm{mol}$ & mole(s) \\
MPa & micropascal(s) \\
MULTRAN & Multicomponent Transport \\
Na & sodium \\
NA & not applicable \\
NAPL & non-aqueous phase liquid \\
PFT & perfluorinated tracer \\
ppm & parts per million \\
PNNL & Pacific Northwest National Laboratory \\
RELAP & Reactive Transport Laplace Inversion \\
s & second(s) \\
Saf-T & Safranin-T \\
SA/V & surface area-to-volume (ratio) \\
SSA & specific surface area \\
STOMP & Subsurface Transport Over Multiple Phases \\
TZIM & thermally degrading zonal isolation material \\
& \\
\hline &
\end{tabular}




\section{Contents}

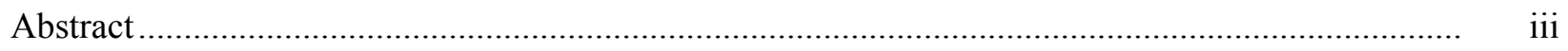

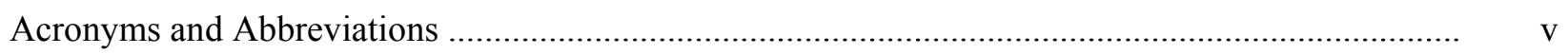

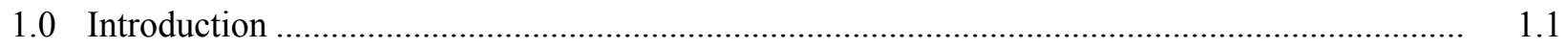

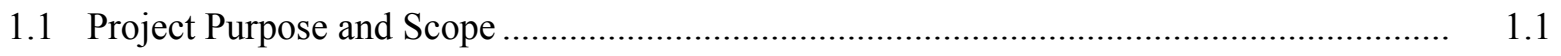

1.2 Report Contents and Organization ........................................................................... 1.2

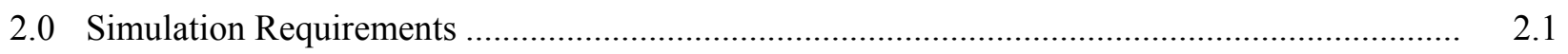

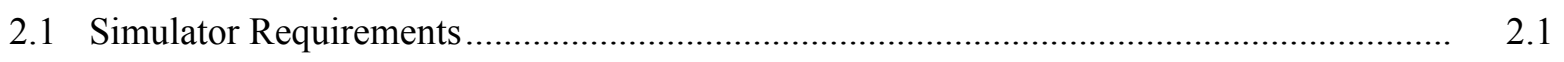

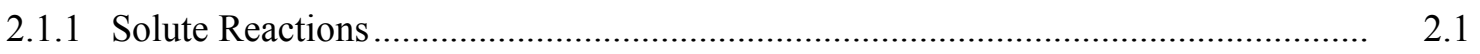

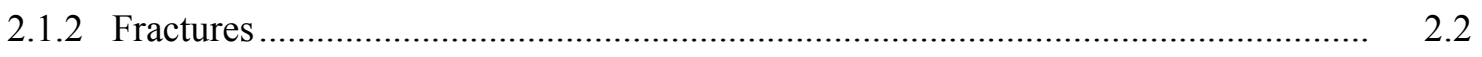

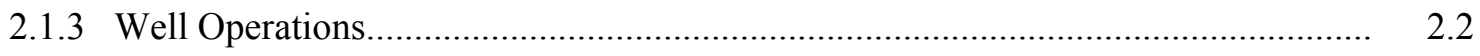

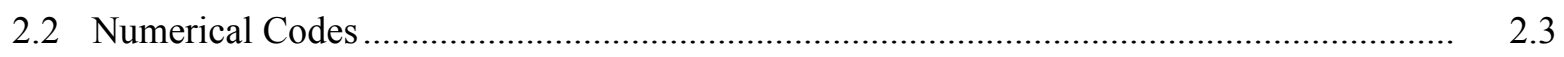

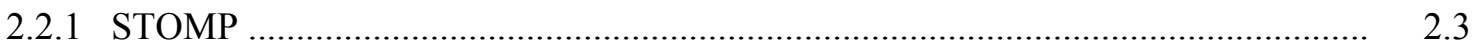

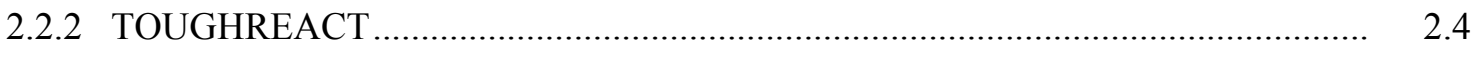

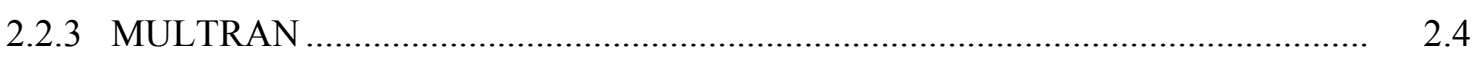

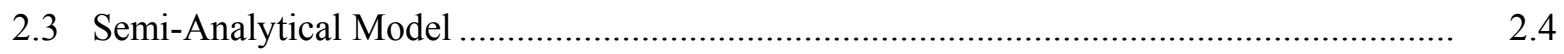

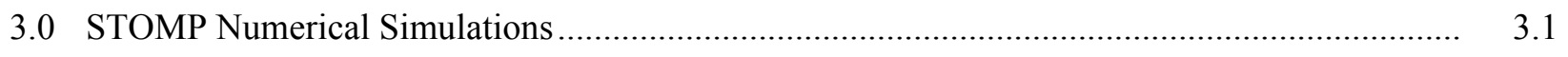

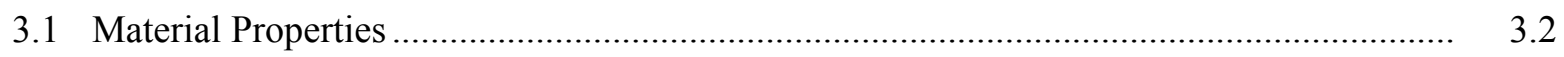

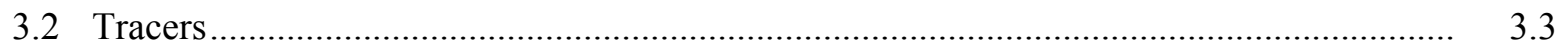

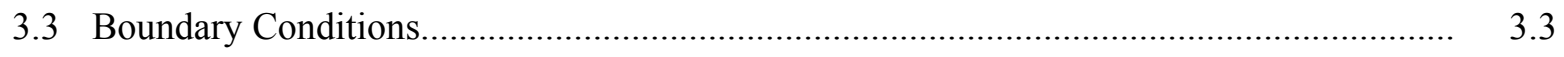

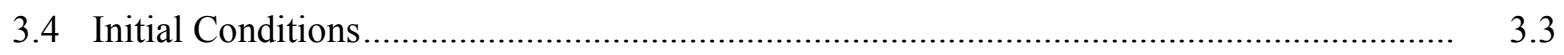

3.5 Operational/Transient Conditions ......................................................................... 3.3

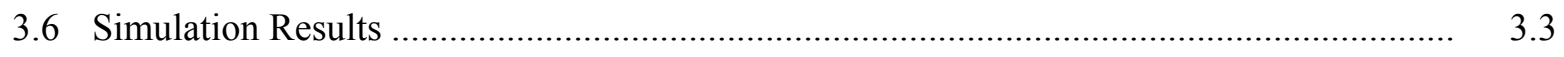

4.0 Semi-Analytical Model for Interwell Tracer Tests..........................................................

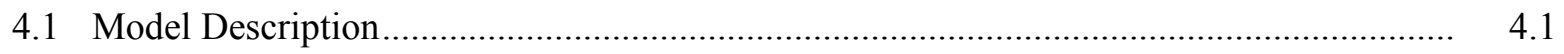

4.2 Evaluation of the Capability of Thermally Degrading Tracers to Predict Thermal
Breakthrough .............................................................................................................

4.2.1 Thermal Breakthrough Concepts and Parameters ................................................ 4.7

4.2.2 Thermal Breakthrough Parameter Sensitivity Cases ............................................. 4.11

4.3 Conservative Tracers with Different Diffusion Coefficients to Estimate Fracture Apertures or Surface Area-to-Volume Ratios ................................................................ 4.19

4.4 Adsorbing Tracers to Estimate Fracture Apertures or Surface Area-to-Volume Ratios ..... 4.22

4.5 Combined Use of Tracers in Interwell Tracer Tests to Estimate Flowing Fracture Spacings

5.0 Multicomponent Numerical Model for Heat and Mass Transport in Interwell and SingleWell Tracer Tests, Including Cation-Exchange Reactions ................................................... 5.1

5.1 Model Description................................................................................................. 5.1

5.2 Temperature as a Tracer in Single-Well Tests to Estimate Flowing Fracture Spacings ...... 5.3 
5.3 Diffusing and Adsorbing Tracers to Estimate Fracture Apertures or Surface Area-toVolume Ratios in Single-Well Tracer Tests.................................................................. 5.6

5.4 The Special Case of Cation-Exchanging Tracers in Single-Well Tracer Tests................... 5.8

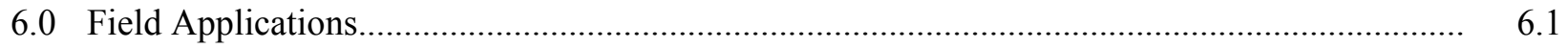

6.1 Soda Lake, Nevada Tracer Test Simulations ............................................................. 6.1

6.1.1 Semi-Analytical Model Analysis ..................................................................... 6.3

6.1.2 Numerical Model.......................................................................................... 6.18

6.1.3 Simulated Single-Well Injection/Withdrawal Tests at Soda Lake ........................ 6.20

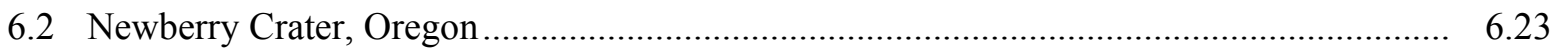

6.2.1 Laboratory Experiments on Selected Tracers..................................................... 6.25

6.2.2 Development of TOUGHREACT Tracer Simulations for Newberry Crater EGS Demonstration................................................................................... 6.33

6.2.3 Tracer Test Operational Support ................................................................ 6.37

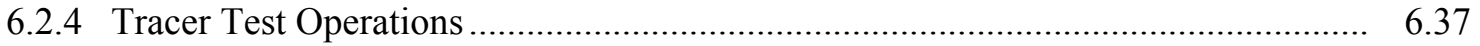

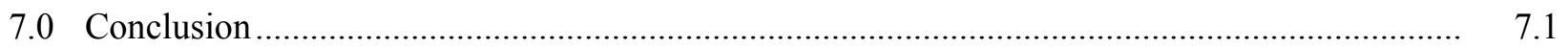

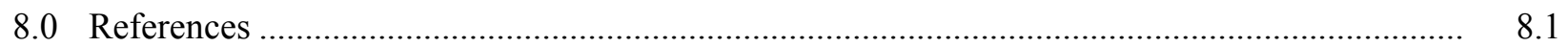




\section{Figures}

3.1 Two-dimensional, radially symmetric, finite difference STOMP grid .................................... 3.1

3.2 Zoomed view of the 2D, radially symmetric, finite difference STOMP grid .......................... 3.2

3.3 Simulated temperatures for an example single-well injection/shut-in/withdrawal test ............. 3.4

3.4 Simulated conservative tracer for an example single-well injection/shut-in/withdrawal test..... 3.5

3.5 Simulated tracer with constant half-life for an example single-well injection/shut-

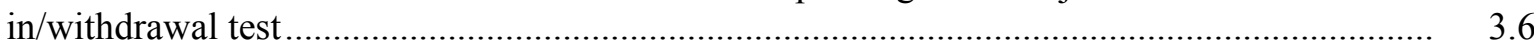

3.6 Simulated tracer with temperature-dependent degradation for an example single-well injection/shut-in/withdrawal test.

3.7 Plot of simulated well concentrations and injection/withdrawal rates for a single-well injection/withdrawal test.

3.8 Simulated tracers and temperatures for an injection/shut-in/withdrawal test ........................ 3.9

3.9 Simulated tracers and temperatures for an injection/shut-in/withdrawal test ........................ 3.10

4.1 Semi-analytical model geometry and key governing mass and heat transport equations ........... 4.1

4.2 Convolution in the Laplace domain of several model segments to obtain a spatially discretized temperature solution as a function of time between an injection and production well

4.3 Simulated temperature as a function of time and fractional distance between the injection well and production well

4.4 Simulated breakthrough curves of a conservative tracer and thermally degrading tracer at three different times after the start of reservoir operations

4.5 Ranges of Arrhenius decay parameters $A$ and $E_{a}$ providing quantifiable interpretations of thermally degrading tracers relative to conservative tracers

4.6 Breakthrough curves of conservative non-decaying and thermally degrading tracers under linear and biradial flow conditions using the model parameters listed in Table 4.2 .........

4.7 Breakthrough curves of conservative non-decaying and thermally degrading tracers from 10 simulations having 20 segments with a standard deviation of log apertures of $0.3 \log$ units

4.8 Breakthrough curves of conservative non-decaying and thermally degrading tracers from 10 simulations having 20 segments with a standard deviation of log fracture spacing of $0.5 \log$ units

4.9 Thermal breakthrough times as a function of $L / 2 b$ for simulations in which fracture apertures were varied by $0.3 \log$ units or fracture spacings were varied by $0.5 \log$ units.

4.10 Tracer breakthrough curves for thermally stable and thermally decaying tracers in simulated tracer tests conducted at different times since the start of cold water injection for cases with a thermal breakthrough time of 45,000 hr and 34,200 hr.

4.11 Example of the prediction of thermal breakthrough time from the y-intercept of a 2nd-order polynomial fit to the log of the ratio of a thermally decaying tracer peak concentration to non-decaying tracer peak concentration at different tracer test times.

4.12 Simulated differences in breakthrough curves of conservative tracers with different matrix diffusion coefficients as a function of fracture half-aperture 
4.13 Simulated differences in breakthrough curves as a function of fracture half-aperture for conservative and adsorbing tracers having matrix diffusion coefficients of $1 \times 10^{-6} \mathrm{~cm}^{2} / \mathrm{s}$ at $25^{\circ} \mathrm{C}$

4.14 Simulated differences in breakthrough curves as a function of fracture half-aperture for conservative and adsorbing tracers having matrix diffusion coefficients of $1 \times 10^{-8} \mathrm{~cm}^{2} / \mathrm{s}$ at $25^{\circ} \mathrm{C}$

5.1 Wellbore temperature histories in single-well tests with $50-\mathrm{hr}$ injection of $25^{\circ} \mathrm{C}$ water into a $250^{\circ} \mathrm{C}$ reservoir followed by 2 -hr rest period as a function of fracture halfaperture, fracture half-spacing, and volumetric flow rate in and out

5.2 Wellbore temperature histories in single-well tests with 50-hr injection and 250-hr injection of $25^{\circ} \mathrm{C}$ water into a $250^{\circ} \mathrm{C}$ reservoir followed by 2 -hr rest period in each case

5.3 Simulated single-well breakthrough curves of conservative tracers with different diffusion coefficients as a function of fracture half-aperture in a $200^{\circ} \mathrm{C}$ reservoir.

5.4 Simulated single-well breakthrough curves of nonsorbing and sorbing tracers with the same diffusion coefficients as a function of fracture half-aperture in a $200^{\circ} \mathrm{C}$ reservoir

5.5 Simulated single-well breakthrough curves of nonsorbing and sorbing tracers in a system with no matrix porosity.....

5.6 Simulated differences in conservative and cation-exchanging tracer breakthrough curves for SA/V values of $10 \mathrm{~cm}^{-1}$ and $20 \mathrm{~cm}^{-1}$

5.7 Simulated single-well breakthrough curves as a function of fracture half-aperture for a naphthalene sulfonate, a halide, a cation-exchanging tracer, and a linear reversibly adsorbing tracer after a tracer injection of $5 \mathrm{hr}$, injection of tracer-free water for $45 \mathrm{hr}$, and a 2 -hr rest period

5.8 Simulated single-well breakthrough curves for a naphthalene sulfonate, a halide, a cation-exchanging tracer, and a linear reversibly adsorbing tracer in a system with a fracture half-aperture of $0.15 \mathrm{~cm}$, but with a matrix CEC of $0.0003 \mathrm{eq} / \mathrm{kg}$

5.9 Simulated single-well breakthrough curves as a function of fracture half-aperture for a naphthalene sulfonate, a halide, a cation-exchanging tracer, and a linear reversibly adsorbing tracer in a system with matrix diffusion and cation exchange parameters similar to those measured in the Newberry Crater laboratory column experiments of Section 6.2.1

5.10 Simulated single-well breakthrough curves as a function of reservoir temperature for a naphthalene sulfonate, a halide, a cation-exchanging tracer, and a linear reversibly adsorbing tracer in a system with matrix diffusion and cation exchange parameters similar to those measured in the Newberry Crater laboratory column experiments of Section 6.2.1

5.11 Simulated single-well breakthrough curves as a function of rest period for a naphthalene sulfonate, a halide, a cation-exchanging tracer, and a linear reversibly adsorbing tracer in a system with matrix diffusion and cation-exchange parameters similar to those measured in the Newberry Crater laboratory column experiments of Section 6.2.1

5.12 Simulated single-well breakthrough curves for the $b=0.05 \mathrm{~cm}$ case of Figure 5.7 with a pulse injection and with a continuous injection over the entire $50 \mathrm{hr}$ of injection.

5.13 Simulated single-well breakthrough curves for the $b=0.55 \mathrm{~cm}$ case of Figure $5.9 \mathrm{but}$ with the addition of fracture fill material that has a cation-exchange capacity of $0.0011 \mathrm{eq} / \mathrm{kg}$, a $K_{d}$ value of $0.5 \mathrm{ml} / \mathrm{g}$, and causes an internal fracture porosity of 0.4

6.1 Soda Lake, Nevada, tracer test site location and well schematic 
6.2 Arrhenius parameters for the thermal degradation of Saf-T determined from laboratory studies at EGI

6.3 Normalized breakthrough curves of 1,6-NDS and Saf-T in the Soda Lake tracer test

6.4 Schematic of single-porosity and dual-porosity conceptual models

6.5 Single- and dual-porosity model fits to the 1,6-NDS and decay-corrected Saf-T breakthrough curves

6.6 Illustration of difference between sorption surface area and heat transfer surface area at a fracture surface

6.7 Schematic of the rim-zone model

6.8 Rim-zone model fits to Saf-T breakthrough curve using different values of the fracture half-aperture $b$.

6.9 TOUGHREACT Soda Lake, Nevada tracer test model results

6.10 Simulated single-well tracer tests with a conservative tracer and a reactive tracer using the TOUGHREACT code

6.11 Results of sensitivity studies for single-well injection/withdrawal tests using the TOUGHREACT code

6.12 Newberry Crater EGS demonstration site showing the Newberry National Volcanic Monument, geothermal leases, and the communities of Bend, Sunriver, Three Rivers, and La Pine

6.13 PNNL process trailer and mixing tank used for tracer tests at Newberry Crater field site

6.14 Lithium and cesium room temperature batch experimental results with synthetic Newberry Crater water chemistry that does not have $\mathrm{K}$ substituted for $\mathrm{Ca}$ and $\mathrm{Mg}$......

6.15 Log-log plots of $\mathrm{Li}$ and $\mathrm{Cs}$ batch experiments at $\sim 1 \mathrm{ppm}, \sim 5 \mathrm{ppm}$, and $\sim 25 \mathrm{ppm}$ comparing sorption in K-substituted water in the mix and in the original Newberry water.......

6.16 NS and $\mathrm{Li}^{+}$breakthrough curves and model fits in laboratory column experiments

6.17 Schematic illustration of rim zone on grains in laboratory column experiments

6.18 Temperature dependence of selectivity coefficient $K_{N a / L i}$ used to simultaneously fit the lithium breakthrough curves of Figure 6.15

6.19 $\mathrm{NS}, \mathrm{Li}^{+}$, and $\mathrm{Cs}+$ breakthrough curves and MULTRAN fits at $225^{\circ} \mathrm{C}$ in the LANL columns

6.20 $\mathrm{NS}$ and $\mathrm{Li}^{+}$breakthrough simulations at temperatures ranging from $25^{\circ} \mathrm{C}$ to $225^{\circ} \mathrm{C}$ assuming LANL column experimental conditions

6.21 Preliminary TOUGHREACT simulations of the $\mathrm{LiBr}$ Trimix experiments using a cation exchange reaction network

6.22 Preliminary example TOUGHREACT simulation of the pentamix $\mathrm{Li}+$ experiment at $225^{\circ} \mathrm{C}$ using a cation-exchange reaction network.

\section{Tables}

3.1 Material properties used in the example simulation

4.1 Model parameters used in RELAP simulations of Figure 4.3 and Figure 4.4 
4.2 Model parameters used in RELAP simulations with 20 model segments ............................ 4.12

4.3 Actual and predicted thermal breakthrough times using the method of Figure $4.11 \ldots \ldots \ldots \ldots \ldots . . . . . .18$

5.1 Model parameters used in MULTRAN simulations of Figure 5.7 through Figure 5.9 .............. 5.11

6.1 Best-fitting RELAP parameters for different conceptual models ........................................... 6.6

6.2 Sensitivity of single-porosity model fits to fracture retardation factor .................................. 6.7

6.3 Sensitivity of dual-porosity model fits with $\emptyset b D m=1 \mathrm{e}-4 \mathrm{~s}-1 / 2$ to fracture and matrix retardation factors

6.4 Sensitivity of dual-porosity model fits with $\emptyset b D m=1 \mathrm{e}-3 \mathrm{~s}-1 / 2$ to fracture and matrix retardation factors

6.5 Rim-zone model parameters, including upper-bound estimates of fracture half-apertures, yielding good fits to the Saf-T breakthrough curve assuming a tuff-specific surface area of $35,000 \mathrm{~cm}^{2} / \mathrm{g}$

6.6 Rim-zone model parameters, including upper-bound estimates of fracture half-apertures, yielding good fits to the Saf-T breakthrough curve assuming a tuff-specific surface area of $100,000 \mathrm{~cm}^{2} / \mathrm{g}$

6.7 Rim-zone model parameters, including upper-bound estimates of fracture half-apertures, yielding good fits to the Saf-T breakthrough curve assuming a tuff-specific surface area of $15,000 \mathrm{~cm}^{2} / \mathrm{g}$.

6.8 Summary of parameters for fitting tracer test with TOUGHREACT V1.2 …....................... 6.21

6.9 Synthetic water chemistries used in the laboratory experiments ............................................ 6.27

6.10 Model parameters used in MULTRAN simulations of laboratory column experiments ........... 6.30

6.11 Concentrations of simulated groundwater and tracers used in trimix experiments ................. 6.34

6.12 Pulse lengths and rates used in trimix experiments .................................................... 6.34 


\subsection{Introduction}

A recent report (MIT 2006) found that electrical power derived from engineered (or enhanced) geothermal systems (EGSs) "represents a large, indigenous resource that could provide base-load electric power and heat that can have a major impact on the United States, while incurring minimal environmental impacts." EGS resources differ from high-grade hydrothermal resources in that they lack sufficient temperature distribution, permeability/porosity, fluid saturation, or recharge of reservoir fluids (MIT 2006). Therefore, quantitative characterization of temperature distributions and the surface area available for heat transfer is necessary for the design and commercial development of a potential EGS site.

This project was a collaborative effort between Brookhaven National Laboratory (BNL), Los Alamos National Laboratory (LANL), Pacific Northwest National Laboratory (PNNL), and the Energy and Geoscience Institute (EGI) at the University of Utah. The project covered a wide array of activities including bench-scale testing of tracers, numerical modeling, interpretation of existing tracer tests, and design and conducting field tracer tests at a demonstration EGS site.

\subsection{Project Purpose and Scope}

The goal of this project is to provide integrated tracer-selection and tracer-interpretation tools to facilitate this characterization. To achieve this goal, this project investigated

- suites of tracers consisting of compounds with different chemical and physical properties that can be injected into wells and will interact in different and measurable ways with the fractured rock matrix (which is the focus of enhanced permeability/porosity for EGS technologies)

- single- and multi-well test designs and corresponding interpretation methods to extract the temperaturedistribution and surface-area information from differences in the tracer concentration versus time histories (i.e., breakthrough curves).

The target tracer suite(s) will include 1) compounds that thermally degrade over a defined range of temperatures, 2) partitioning/adsorbing tracers that have a variety of interaction characteristics with rock surfaces, and 3) conservative tracers that provide the basis for differential interpretation. From this tracer information, this project will develop 1) tracers that can be reliably applied to provide quantitative information about temperature distribution and fracture surface area, 2) tracer test designs (both singlewell and multi-well) to exploit the use of these tracers, and 3) interpretive methods whereby this information will provide practical guidance to improve heat-extraction capabilities.

The use of thermally degrading and adsorbing tracers in geothermal applications was first suggested in model simulations conducted by LANL during the Fenton Hill Hot Dry Rock geothermal project (Robinson et al. 1988; Nicol and Robinson 1990; Chemburkar et al. 1991). LANL has subsequently used adsorbing tracers and conservative tracers with different diffusion coefficients to estimate surface area-tovolume (SA/V) ratios in fractured rock groundwater systems (Reimus and Callahan 2007; Reimus 2010). Differential tracer transport has also been used by BNL and others in oil field characterization and in characterization of groundwater and contaminated soil systems (Sennum et al. 1989; Tang 1995; Brusseau et al. 2003). BNL has also developed methods for seven perfluorinated tracer (PFT) compounds as nonreactive tracers in numerous applications from atmospheric transport to underground leak detection (Watson et al. 2007). This project initially focused on tracer development with the application of PFT 
tracers to geothermal systems and evaluation of encapsulated PFTs that would release tracers at a targeted reservoir temperature (see Reimus et al. 2011). After the 2011 DOE Geothermal Technology Program (GTP) midyear review of this project and subsequent discussions with the DOE's GTP, project emphasis was shifted to interpretive tool development, testing, and validation.

The project developed and applied models for describing interactions of tracers with EGS reservoirs in various tracer test configurations. The modeling work extended the theoretical framework that is already used successfully in groundwater applications. Forward modeling was used to select tracers by identifying optimal thermal and surface adsorption properties for geothermal applications, and to evaluate the potential effectiveness of experimentally determined tracer properties in geothermal settings. Integrating modeling with laboratory experiments will make certain that models are improved and validated by the experimental data and that experiments address key modeling needs. Inverse modeling will be used to interpret field tracer transport behavior, providing a method to estimate reservoir properties and their uncertainties.

Various numerical models described in this study were applied by PNNL and LANL to bench-scale batch and column testing results of conservative and reactive tracers with different mineralogy, temperature regimes, and flow rates. These models were also used to analyze an existing field reactive and conservative tracer test data set at a naturally fractured geothermal site at Soda Lake, Nevada. The modeling was also used to design the reactive and conservative tracer tests for an EGS demonstration site near Newberry Crater, Oregon, that was led by Alta Rock Energy, Inc. PNNL and EGI also assisted in conducting the tracer testing during this EGS demonstration that was conducted from October to December 2012. The numerical models developed on this project for the Newberry Crater EGS test can also be used for interpretation of the tracer test results during flowback operations planned for the spring of 2013.

\subsection{Report Contents and Organization}

The purpose of this report is to describe the model development for simulating single- and multi-well tracer tests for characterizing EGS sites. The ensuing chapters of this report contain a description of the simulation requirements, selected codes, examples of preliminary scoping results for both numerical and analytical simulations, and test cases based on two field sites. After completing the initial model development for scoping simulations, models were built based on laboratory testing of and field data for candidate tracers for field test sites, including designing and conducting reactive and conservative tracer tests at an EGS demonstration site. 


\subsection{Simulation Requirements}

This chapter describes the features and capabilities required for simulating single- and multi-well tracer tests targeted at characterizing EGS sites. The selection and development of numerical and analytical codes, along with procedures for code testing, are also discussed.

\subsection{Simulator Requirements}

The geothermal systems simulated as part of this project are assumed to be saturated, single-phase aqueous systems (i.e., no gas/steam phase). In addition to water flow, the codes to be used for simulating tracer transport must account for energy transport (conduction and advection) and mass transport (advection, dispersion, and diffusion) as well as relevant couplings between mass and energy transport (for thermally degrading tracers). At this time, we do not anticipate the need for simultaneous mechanical stress calculations coupled with mass and energy transport calculations (e.g., mechanical opening of fractures and resultant changes in fracture $\mathrm{SA} / \mathrm{V}$ ratio as a result of cooling of the rock mass), because these effects will be treated as unknowns that will be interrogated by the tracers. Additional solute transport requirements for simulating reactive tracers are discussed below, as are requirements for simulating fractures and well functions.

\subsubsection{Solute Reactions}

Simulating the transport behavior of a suite of tracers for EGS reservoir characterization may require the ability to simulate tracer sorption, thermal degradation, diffusion, and possible reaction of a tracer to form reaction products. The types of tracer transport behavior to be simulated, along with the required parameters, will depend on the results of laboratory or relevant field experiments. Initial scoping simulations of differing transport behaviors were conducted over a range of property values to help guide laboratory experiments and identify properties that would be helpful in characterizing EGS sites.

Tracer sorption could include equilibrium sorption with a linear isotherm $\left(\mathrm{K}_{d}\right)$. More complex sorption reactions, such as non-equilibrium or nonlinear isotherms (e.g., Freundlich or Langmuir nonlinear isotherms), may be needed, depending on the results of laboratory experiments for candidate tracers.

In addition to sorption, cation transport can be simulated using cation-exchange reactions. Requirements for simulating cation-exchange reactions include the cation-exchange capacity (CEC) of the reservoir and fracture surfaces, cation selectivity coefficients, initial cation concentrations on the material, and aqueous concentrations of the major cations (see Appelo 1994a, 1994b).

Tracer degradation could include simple constant first-order decay (i.e., half-life) or more complex thermally degrading tracers as described by Adams and Davis (1991) and Rose and Adams (1994). The behavior of thermally degrading tracers has been modeled by the above authors for fluorescein and rhodamine tracers using the Arrhenius equation:

$$
k=A e^{-E_{a} / R T}
$$


where

$$
\begin{array}{ll}
\mathrm{k} & =\text { rate coefficient }(1 / \mathrm{s}) \\
\mathrm{A} & =\text { pre-exponential constant }(1 / \mathrm{s}) \\
\mathrm{E}_{\mathrm{a}} & =\operatorname{activation} \text { energy }(\mathrm{J} / \mathrm{mol}) \\
\mathrm{R} & =\text { ideal gas constant }(8.314 \times 10-3 \mathrm{~kJ} /(\mathrm{mol} \bullet \mathrm{K}) \\
\mathrm{T} & =\text { temperature }(\mathrm{K})
\end{array}
$$

Another type of tracer that could be useful in characterizing EGS sites is a thermally stable solute that is encapsulated in a submicron-diameter shell that can undergo thermal degradation, thereby releasing the solute when the particle experiences a time-temperature history that causes the shell to breach. The encapsulation of PFTs was started as part of the laboratory experiments for this project but was not completed. Modifications of the existing codes would be required to simulate the transport of submicron solutes and release of encapsulated tracers at a threshold temperature based on laboratory test results.

\subsubsection{Fractures}

Fracture/fault characteristics of density, spacing, and, more importantly, the SA/V ratio, are key parameters for understanding fluid flow and heat transfer in EGS reservoirs. The tracers and tracer tests will be designed to interrogate the subsurface to obtain information about these unknown parameters. Thus, the simulators used for tracer test design and interpretation must not be excessively constrained by assumptions about fracture/fault geometry to avoid introducing biases into the designs or interpretations. Relatively simple representations of fracture networks, such as the use of characteristic fracture apertures and fracture spacing, have been used in previous studies and are probably sufficient for achieving design and interpretation objectives.

O'Sullivan et al. (2001) surveyed a broad range of researchers that had simulated geothermal reservoirs at locations around the globe. Based on the results of this survey, fractures and fracture networks have been simulated as either discrete fracture networks or with dual-porosity/dual-continuum models (e.g., Warren and Root 1963). For simulating discrete fracture networks, subgridding or irregular gridding techniques have been used to reduce the grid size and computational requirements of the problem (see O'Sullivan et al. 2001 for examples). Dual-porosity or multi-continuum representations of fractures have been used extensively in geothermal simulations because of their lower computational requirements (e.g., Pruess and Narasimhan 1982) and the general uncertainty in fracture network characterization.

\subsubsection{Well Operations}

The ability of a code to represent wells is important because the introduction and sampling for tracers will generally occur via such subsurface penetrations. Typically, in the code, the well injection/ withdrawal flow rates can be specified directly, rather than requiring knowledge of the time course of well pressures. The capability of a code to calculate vertically variable fluxes across a well screen (given a total discharge amount) because of permeability heterogeneities is also important. However, well functions are difficult to implement in variable density systems because of the nonlinearity of the problem, for which solution convergence can be difficult.

Codes that provide grids with two-dimensional (2D) radial symmetry are needed to simulate singlewell injection/withdrawal tests with relatively simple hydrostratigraphy, such as will be used in initial 
scoping calculations. Multiple injection/withdrawal wells or complex hydrostratigraphy/heterogeneities cannot be accurately simulated with $2 \mathrm{D}$ radially symmetric grids and will require development of threedimensional (3D) model domains.

\subsection{Numerical Codes}

This section describes the numerical codes that were used in simulating injection/withdrawal tracer tests at EGS sites. Semi-analytical codes developed for this effort are described in Chapters 4.0 and 5.0. Three numerical codes-Subsurface Transport Over Multiple Phases (STOMP), TOUGH, TOUGHREACT, and Multicomponent Transport (MULTRAN)_-are described below as candidates for use in simulating tracer tests at EGS sites. A semi-analytic model, Reactive Transport Laplace Inversion (RELAP), is also described below for use in rapid scoping calculation purposes.

The STOMP code was selected because it has many of the capabilities described in Section 2.1, it was familiar to some of the researchers on this project, and the source code is available for customization. The TOUGH2 (Pruess et al. 1999) and TOUGHREACT (Xu et al. 2006, 2012) codes were also used in this project because of their capabilities and wide adoption by geothermal researchers as discussed below.

While example cases and capabilities of the STOMP code are provided in the Chapter 3.0 below, examples of TOUGHREACT runs are not provided, because numerous geothermal test cases are documented in the TOUGH2 (Pruess et al. 1999) and TOUGHREACT User's Manual (Xu et al. 2006, 2012) and in the references provided below. TOUGHREACT runs are described in later sections of this report as applied to the Soda Lake, Nevada and Newberry Crater, Oregon tracer test simulations.

\subsubsection{STOMP}

The STOMP code is a finite difference, multi-fluid, flow and transport code developed by PNNL (White and Oostrom 2000, 2006) and is described online at the following site: http://stomp.pnl.gov. The code has multiple operational modes that support certain combinations of energy transport, non-aqueous phase liquids (NAPLs), gases, and water. STOMP has been enhanced in recent years for use in supercritical $\mathrm{CO}_{2}$ injection simulations for assessing greenhouse gas sequestration. STOMP has a dualcontinuum feature that can be used to represent fractures (implemented in selected modes), but does not currently have a non-equilibrium dual-continuum capability implemented in the energy modes. The STOMP well function is also not currently implemented in the energy mode.

As part of this project, a thermal degradation function for tracers using the Arrhenius equation (as described above) was recently implemented in STOMP. Currently, the STOMP code can be used for simulating injection/withdrawal tests for thermally degrading tracers (see example Chapter 3.0). The STOMP code also accommodates 2D radially symmetric grids that are efficient (compared to 3D grids) for simulating single-well injection/withdrawal tests. Discrete fractures can be simulated with highresolution, variable grid spacing around fracture zones, and higher permeability can be applied to represent the fractures. 


\subsubsection{TOUGHREACT}

TOUGH2 (Pruess et al. 1999) and the updated version for reactive transport, TOUGHREACT (Xu et al. 2006, 2012), have been used for simulating geothermal systems at a large number of sites (e.g., O'Sullivan et al. 2001). TOUGH2 and TOUGHREACT use an integrated finite-difference approach that provides for unstructured grids. In addition to fluid, solute, and energy flow, TOUGH2 and TOUGHREACT provide a multiple interacting continua (MINC) method for simulating fractures (see Pruess 1983, 1992), which is an extension of the dual-porosity method described by Warren and Root (1963). The survey of geothermal simulations by O'Sullivan et al. (2001) showed that many sites have applied the MINC method for simulating fracture systems. Fayer et al. (2009) have used TOUGHREACT with the MINC method for simulating thermally degrading tracers in injection tests. TOUGHREACT V1.2 (Xu et al. 2006) was used in the initial portion of this study and TOUGHREACT V2.0 was used later in this study shortly after it was released (Xu et al. 2012).

\subsubsection{MULTRAN}

The MULTRAN computer model (Sullivan et al. 2003) was used to conduct exploratory simulations to determine the potential for using cation-exchanging tracers to interrogate surface area in both multiwell and single-well tracer tests in EGS reservoirs. It simulates the transport of cation-exchanging tracers by solving mass-action equations for cation-exchange equilibria simultaneously with the transport equations. Like STOMP and TOUGHREACT, MULTRAN can simultaneously solve heat and mass transport equations, and it is capable of simulating the flow interruptions and flow reversals associated with single-well tracer tests, which are difficult to account for with analytical or semi-analytical models. MULTRAN also accounts for the temperature dependence of tracer diffusion coefficients, and it can account for the temperature dependence of reaction rate constants or equilibrium constants of adsorption reactions. This model and its applications are described in more detail in Chapter 5.0.

\subsection{Semi-Analytical Model}

A semi-analytical heat and mass transport model, RELAP (Reactive Transport Laplace Inversion), was developed to conduct rapid scoping calculations of responses of thermally degrading, diffusing, and adsorbing tracers in interwell tracer tests at EGS sites (Reimus 2010). The model is based on a semianalytical computer model for solute transport in dual-porosity media (Reimus et al. 2003). The RELAP model combines the Laplace-domain dual-porosity transport equations derived by Maloszewski and Zuber (1984, Appendix; 1985) with Laplace-domain transfer functions that describe a finite-pulse injection, wellbore mixing, and recirculation (although the latter two features were not used here). It can also simulate linear, reversible sorption (either rate-limited or equilibrium). Maloszewski and Zuber (1984, Appendix; 1985) assumed that tracer transport in fractures was described by the one-dimensional (1D) advection-dispersion equation with 1D diffusion occurring into the surrounding matrix perpendicular to the flow direction in fractures. This model and its applications are described in more detail in Chapter 4.0, which also goes into considerable detail about how tracers with different properties might be used to interrogate reservoir characteristics such as SA/V ratios (or fracture half-apertures) and thermal breakthrough times in interwell tracer tests. 


\subsection{STOMP Numerical Simulations}

This chapter describes some example simulations using the STOMP code with the water, air, and energy mode. A non-equilibrium, dual-continuum model had not been implemented in the energy modes of STOMP at the time of this study, so discrete fractures were simulated with grid refinements as described below.

The initial STOMP simulations were developed for scoping to investigate single-well injection/withdrawal tests with thermally degrading tracers. Two-dimensional, radially symmetric, finite difference grids were constructed with the grid refined around discrete horizontal and vertical fractures. These grids had a $0.25-\mathrm{m}$ horizontal node spacing near the injection/withdrawal well (0 to $50 \mathrm{~m}$ ) with larger grid spacing toward the outer radial boundary (from 50 to $500 \mathrm{~m}$ ) as shown in Figure 3.1. Vertical grid spacing ranged from $0.001 \mathrm{~m}$ within and adjacent to the fracture zone, grading to $0.25 \mathrm{~m}$ away from the fracture zone. Simulations were initially constructed with multiple horizontal and vertical fractures; however, the computational requirements for running these simulations with the large number of nodes needed to resolve these multi-fracture simulations were excessive, so single-fracture simulations were constructed. Different horizontal fracture spacing can be investigated with these single-fracture simulations by adjusting the domain.

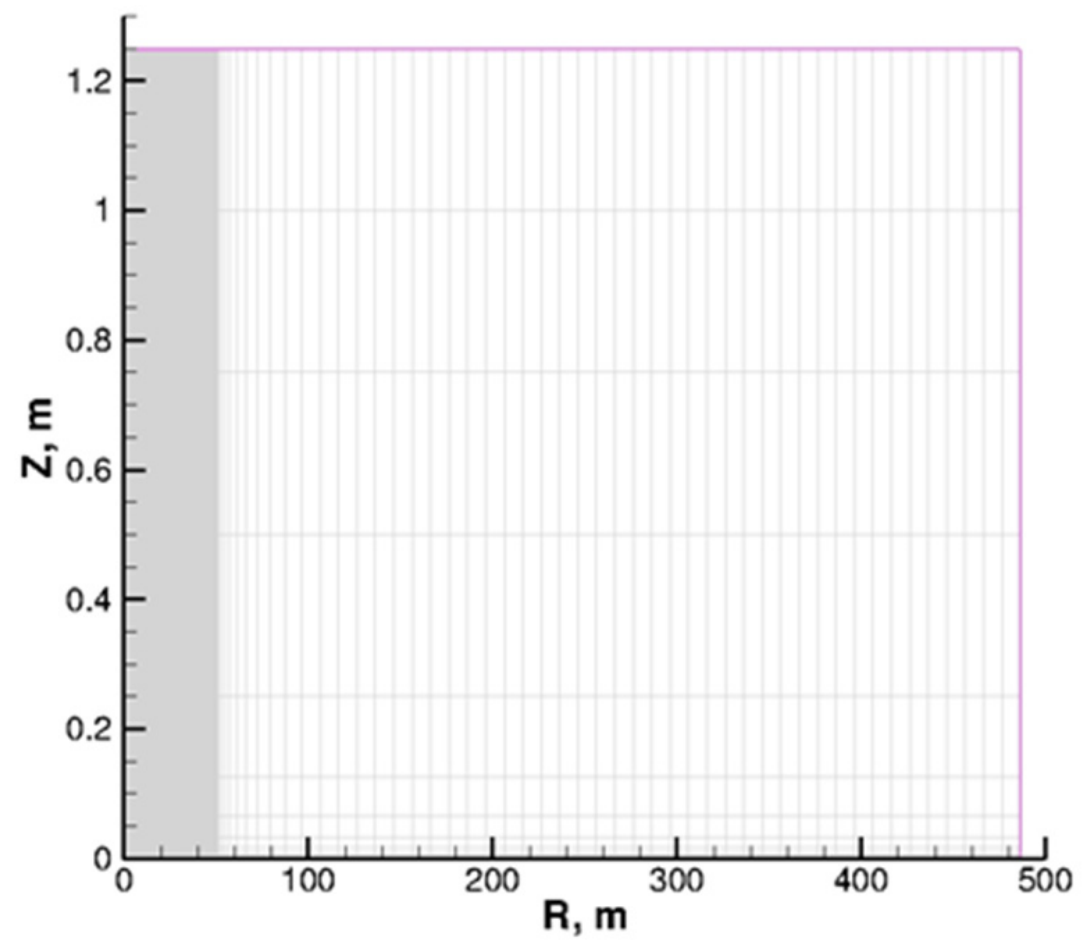

Figure 3.1. Two-dimensional, radially symmetric, finite difference STOMP grid. The grid is refined near the injection/withdrawal well $(\mathrm{R}=0$ to $50 \mathrm{~m})$ boundary and along the bottom (to represent a horizontal fracture zone). See Figure 3.2 also. 


\subsection{Material Properties}

As shown in Figure 3.2, two zones were created representing the bedrock zone for most of the domain and a thin, higher permeability zone at the bottom representing a horizontal fracture. For the example case described in this section, the bedrock zone permeability was set at $1.5 \times 10^{-3}$ darcy with the fracture zone permeability 10,000 times greater at 15 darcy. In addition, the porosity of the bedrock zone was set at $10 \%$ with the fracture zone porosity greater at $40 \%$. Other material properties used in this simulation are listed in Table 3.1 .

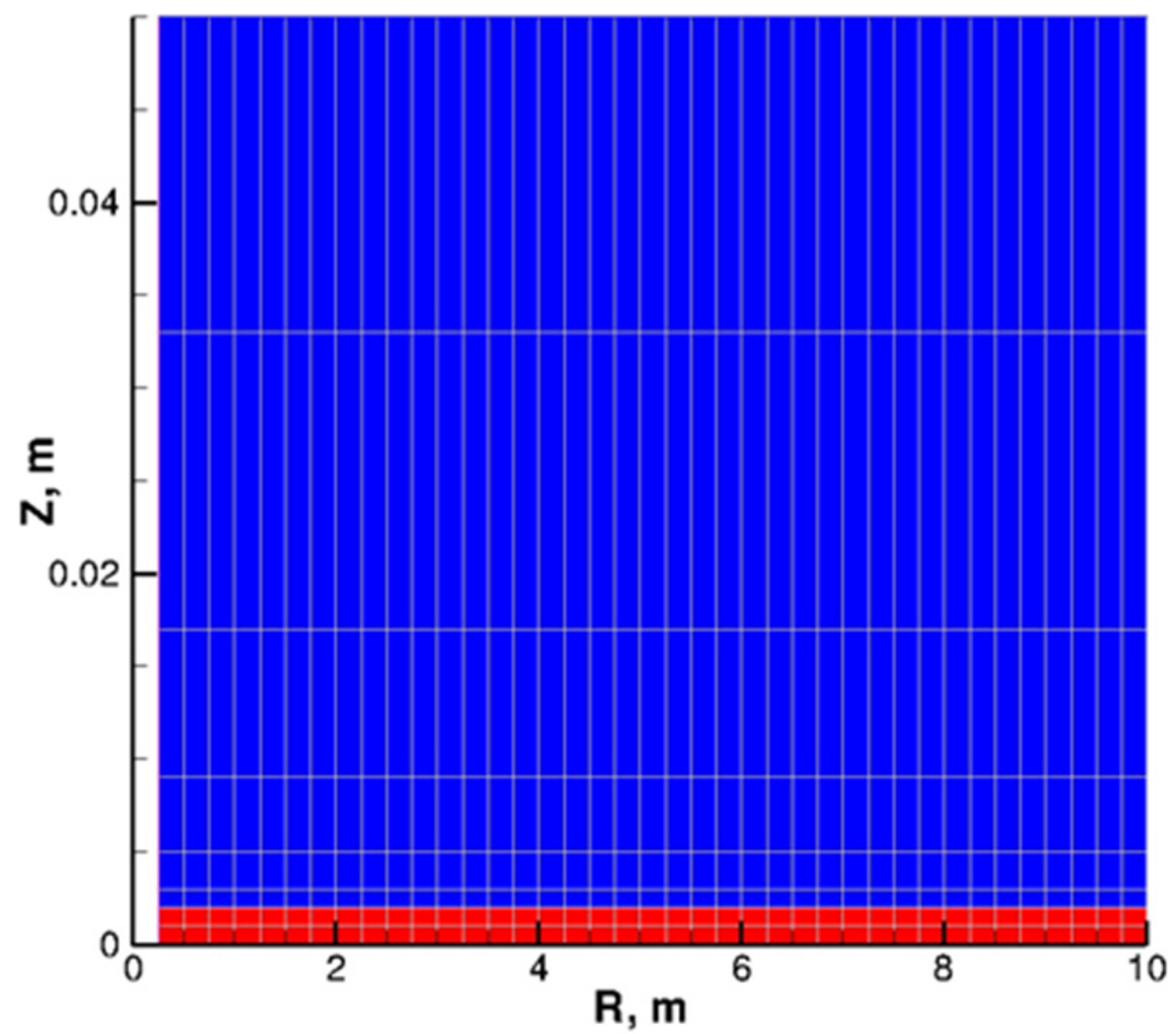

Figure 3.2. Zoomed view of the $2 \mathrm{D}$, radially symmetric, finite difference STOMP grid. The blue color is the bedrock zone, and the red color is the fracture zone.

Table 3.1. Material properties used in the example simulation.

\begin{tabular}{llcccc}
\hline Zone & $\begin{array}{c}\text { Permeability } \\
\text { (isotropic) }\end{array}$ & Porosity & $\begin{array}{c}\text { Rock } \\
\text { Compressibility }\end{array}$ & $\begin{array}{c}\text { Thermal } \\
\text { Conductivity } \\
\text { (isotropic) }\end{array}$ & Specific Heat \\
\hline Bedrock & $1.5 \times 10^{-3}$ darcy & 0.10 & $1.0 \times 10^{-9} 1 / \mathrm{Pa}$ & $3.3 \mathrm{~W} /(\mathrm{m} \mathrm{K})$ & $816 \mathrm{~J} /(\mathrm{kg} \mathrm{K})$ \\
Fracture & 15 darcy & 0.40 & $1.0 \times 10^{-9} 1 / \mathrm{Pa}$ & $3.3 \mathrm{~W} /(\mathrm{m} \mathrm{K})$ & $816 \mathrm{~J} /(\mathrm{kg} \mathrm{K})$ \\
\hline
\end{tabular}




\subsection{Tracers}

Three different tracers - designated as T1, T2, and T4-were set up for these simulations. All three tracers are nonsorbing with aqueous molecular diffusion rates of $7.8 \times 10^{-10} \mathrm{~m}^{2} / \mathrm{s}$ at $20^{\circ} \mathrm{C}$. Longitudinal dispersivity for these tracers was set to $0.2 \mathrm{~m}$ and $0.02 \mathrm{~m}$ transverse dispersivity. Tracer $\mathrm{T} 1 \mathrm{is}$ a conservative tracer with no decay. Tracer $\mathrm{T} 2$ has a constant first-order decay rate with a half-life of $0.5 \mathrm{yr}$. Tracer T4 is a synthetic, thermally degrading tracer with the Arrhenius constants selected to yield a 0.5 -yr half-life at $200^{\circ} \mathrm{C}$. The Arrhenius parameters for tracer T4 are $\mathrm{k}=2.9 \times 10^{8} 1 / \mathrm{s}$ and $\mathrm{A}_{\mathrm{e}}=$ $1.433 \times 10^{5} \mathrm{~J} / \mathrm{mol}$.

\subsection{Boundary Conditions}

The top and bottom of the simulation domain were set to no-flow conditions for fluid, heat, and solutes. At the bottom of the innermost $(\mathrm{R}=0)$ and outermost $(\mathrm{R}=500 \mathrm{~m})$ boundaries, the pressures were initially set to $20.0 \mathrm{MPa}$ to represent fluid pressures at approximately a 2-km depth. Pressures on the sides of the domain were calculated at hydrostatic pressures from the 20.0-MPa specified base pressure. Temperatures on the inner and outer boundaries, and within the domain, were initially set to $200^{\circ} \mathrm{C}$. The pressures and temperatures for these simulations were chosen so that the water would be in the liquid phase only.

\subsection{Initial Conditions}

Initial temperatures within the domain were set to $200^{\circ} \mathrm{C}$, and all tracer concentrations were set to zero. Initial pressures were calculated from the results of a quasi-steady state run using the boundary value pressures described above.

\subsection{Operational/Transient Conditions}

The simulation was configured for an initial 30-d injection period with pressures specified on the injection well side of the domain $(\mathrm{R}=0)$ calculated from a base pressure of $20.1 \mathrm{MPa}$. The injection temperature was cooler than the formation fluid and set to $150^{\circ} \mathrm{C}$. Injection concentrations of the tracers were set to 1 . Following the injection period was a $30-\mathrm{d}$ shut-in period with the boundary condition at the injection side $(\mathrm{R}=0)$ set to no-flow for fluid, temperature, and solutes. A 30-d withdrawal period was started at the end of the shut-in period (elapsed simulation time of $60 \mathrm{~d}$ ) with pressures set on the injection well side of the domain calculated from a base pressure of $19.9 \mathrm{MPa}$. Fluid temperatures entering the outermost boundary $(\mathrm{R}=500 \mathrm{~m})$ during the withdrawal period were set to $200^{\circ} \mathrm{C}$, and the inflowing solute concentrations were set to zero.

\subsection{Simulation Results}

Figure 3.3 shows the simulated temperature field results at selected times for the injection ( 0 to $30 \mathrm{~d})$, shut-in ( 30 to $60 \mathrm{~d}$ ), and withdrawal (60 to $90 \mathrm{~d}$ ) periods. Concentrations for the conservative tracer (T1) for these times are shown in Figure 3.4. Simulated concentrations for the tracer (T2) with a constant 0.5-yr half-life are shown in Figure 3.5, and the simulated concentrations for the tracer (T4) undergoing temperature-dependent (Arrhenius) degradation are shown in Figure 3.6. 

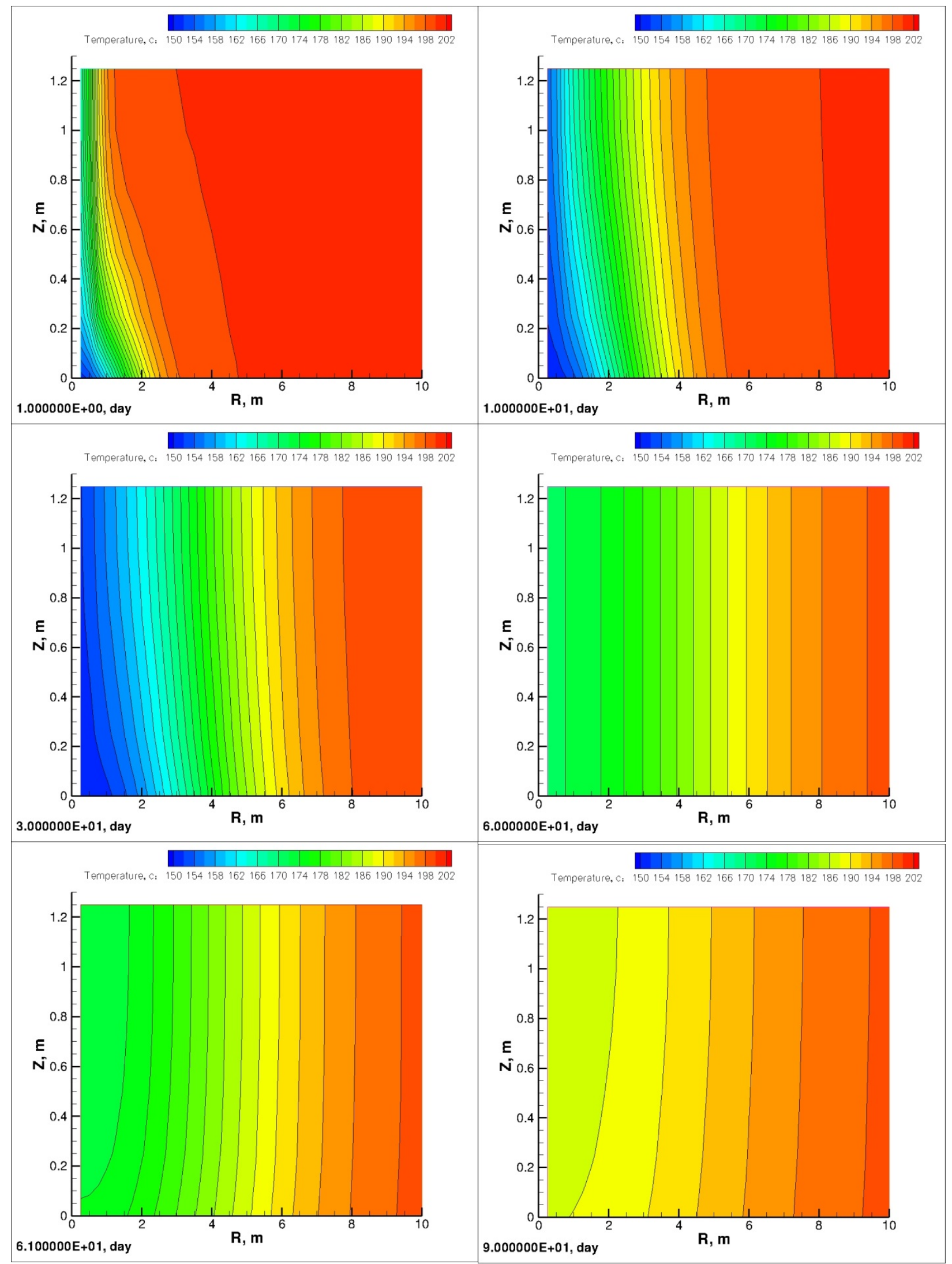

Figure 3.3. Simulated temperatures for an example single-well injection/shut-in/withdrawal test. 


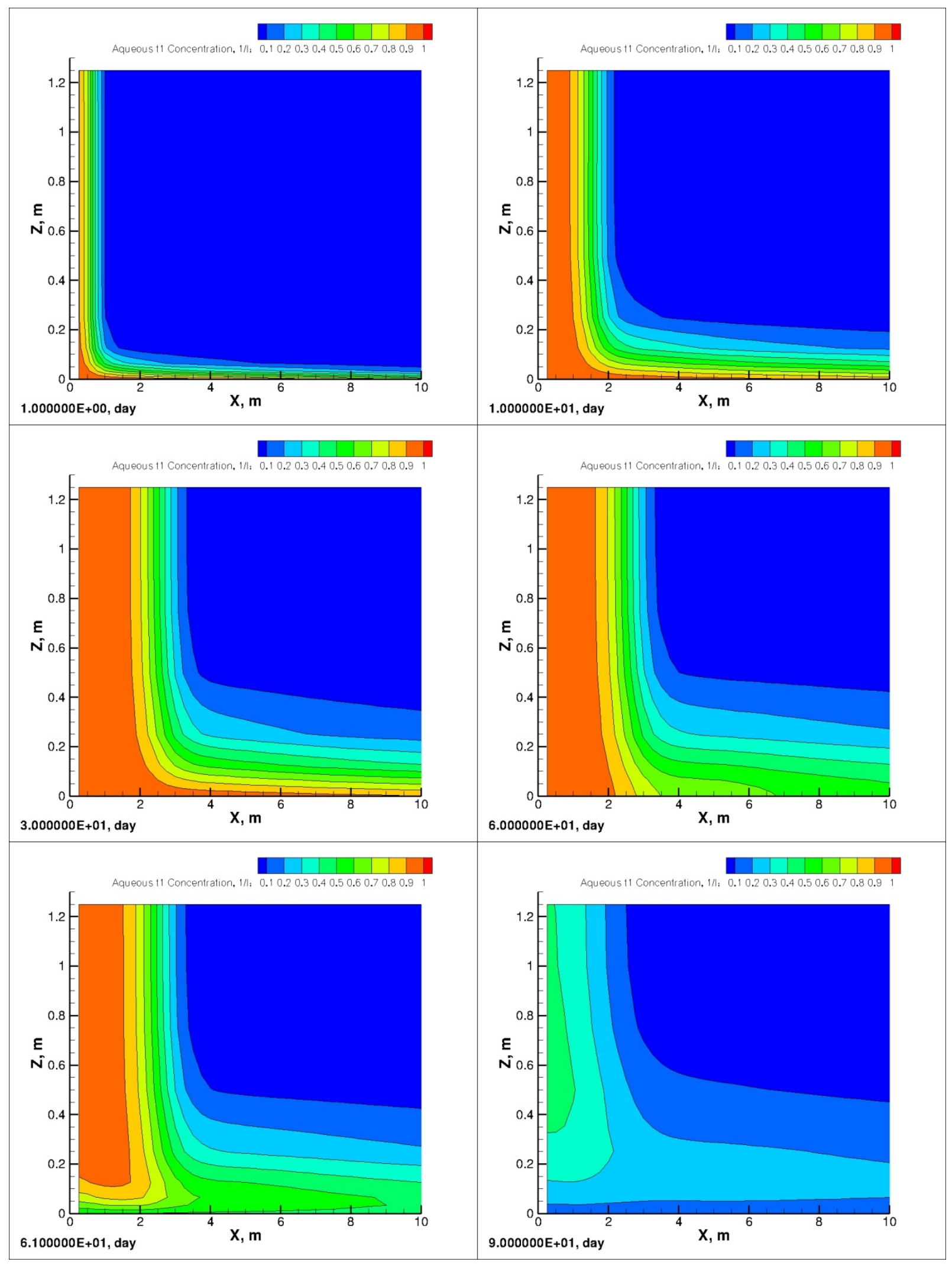

Figure 3.4. Simulated conservative tracer for an example single-well injection/shut-in/withdrawal test. 


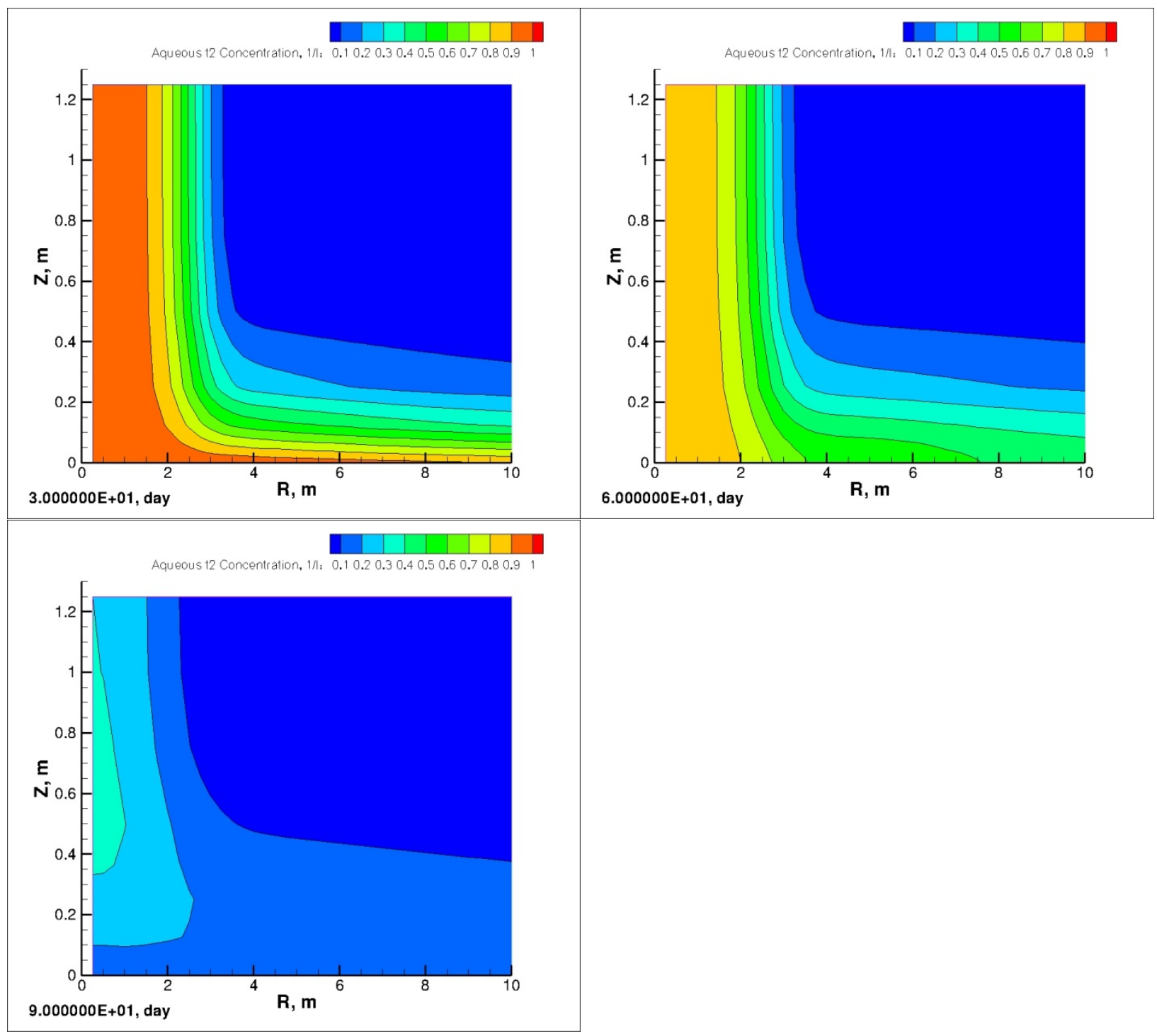

Figure 3.5. Simulated tracer with constant half-life for an example single-well injection/shutin/withdrawal test. 


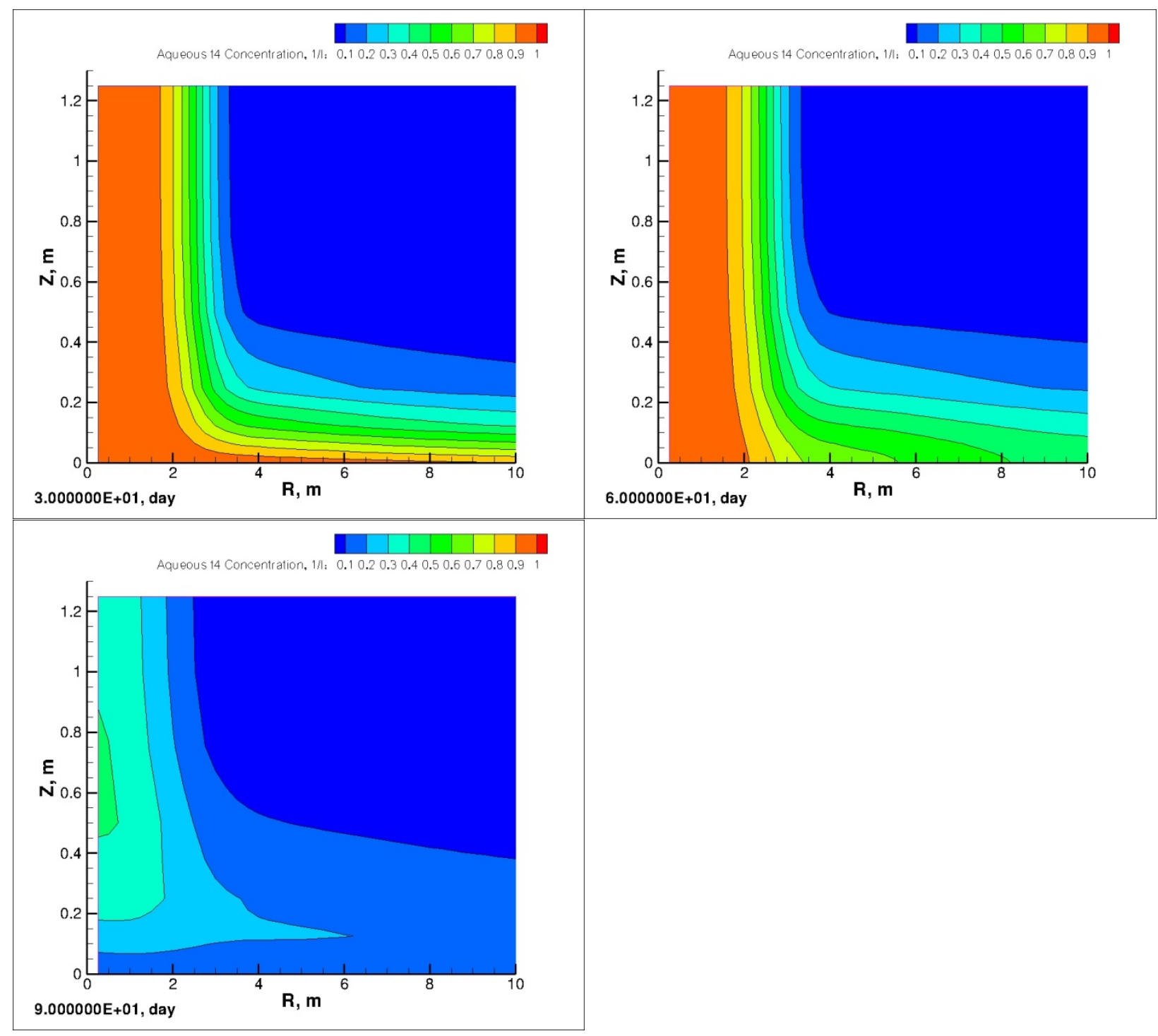

Figure 3.6. Simulated tracer with temperature-dependent degradation for an example single-well injection/shut-in/withdrawal test.

A trend plot showing tracer concentrations within the well and the well flux rates is shown in Figure 3.7 for the three operational periods (injection, shut-in, withdrawal). Because a constant pressure boundary condition is used to simulate a well, the well flux rates take a while to stabilize during the start of the injection and withdrawal stages (see Figure 3.7). Applying temporal changes in the boundary pressures would achieve a more uniform flux rate without using a well model. In addition, the injection rates are slightly lower than the withdrawal rates with these boundary pressures. Because a well function was not built into the energy mode for STOMP at the time of this study, these pressures can be adjusted based on a few trial and error runs with the same material properties and boundary pressures.

Figure 3.8 and Figure 3.9 show simulated tracer concentrations and temperatures for nodes within the model domain during the injection, shut-in, and withdrawal stages. The node locations were selected within the fracture zone $(\mathrm{Z}=0 \mathrm{~m})$ and at the farthest distance away from the fracture zone $(\mathrm{Z}=1.125 \mathrm{~m})$ 
near the upper model boundary. Figure 3.8 shows the results near the injection/withdrawal well $(\mathrm{R}=0.375 \mathrm{~m})$ and Figure 3.9 shows the results $2.625 \mathrm{~m}$ away from the injection/withdrawal well.

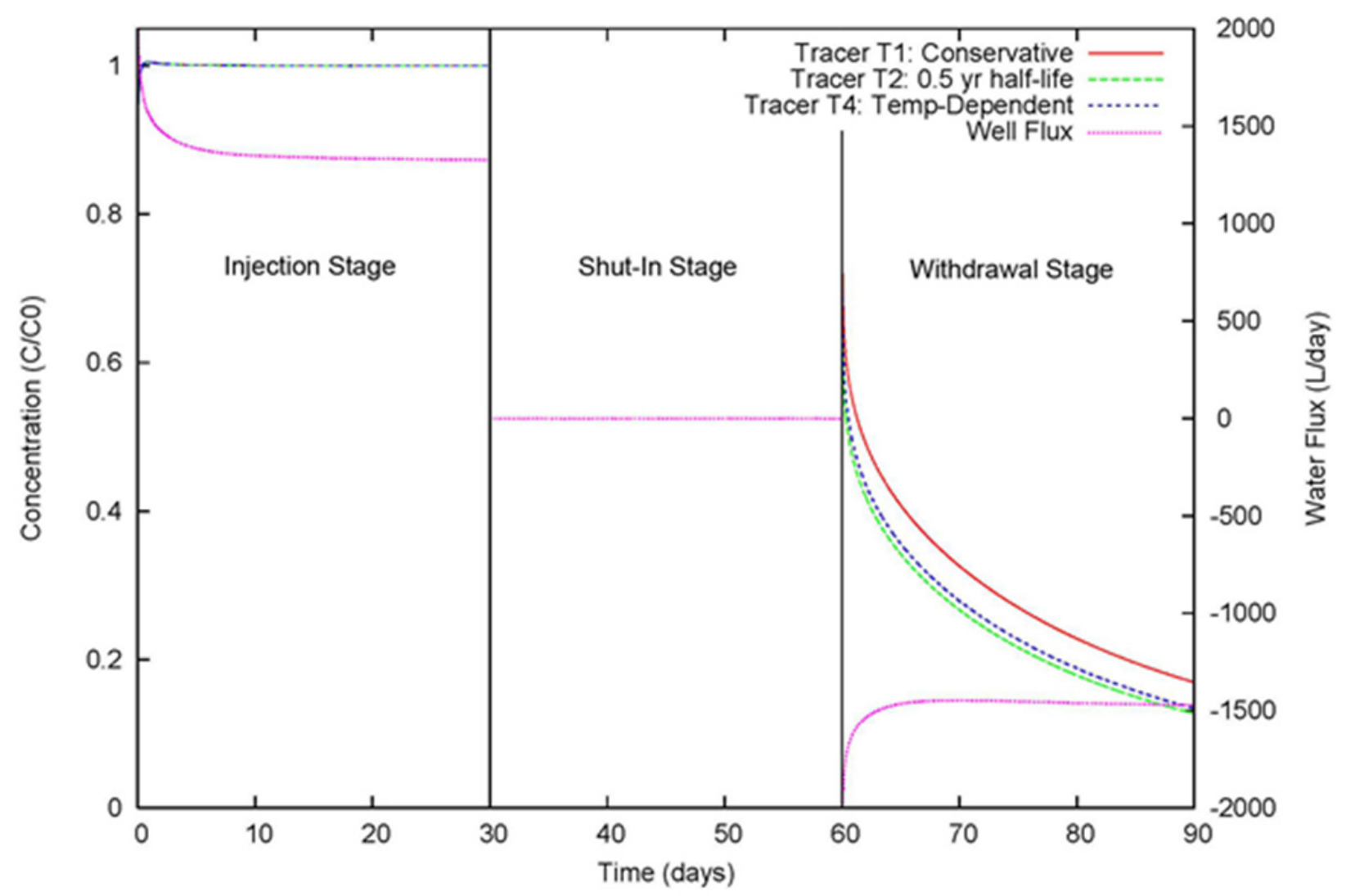

Figure 3.7. Plot of simulated well concentrations and injection/withdrawal rates for a single-well injection/withdrawal test.

The computational requirements for these STOMP simulations with discrete fractures in a single injection/withdrawal well operation are long (e.g., weeks for a single run) because of the small time steps required for convergence. The majority of the run time is consumed during the simulation of the first few days because of the steep temperature gradients near the injection/withdrawal well. Larger time steps can be taken during the later portion of the simulation period. 


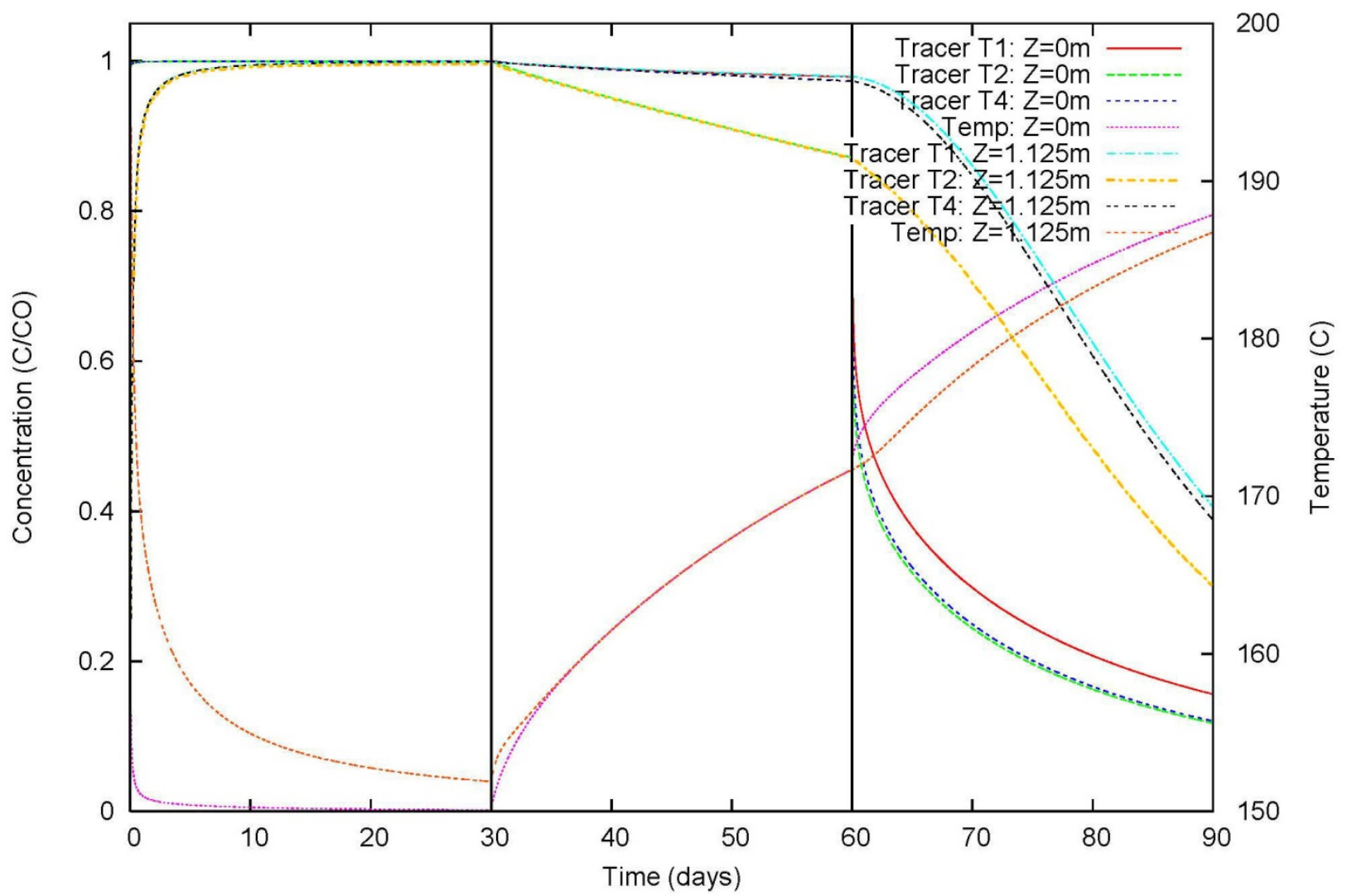

Figure 3.8. Simulated tracers and temperatures for an injection/shut-in/withdrawal test. Results are for near-well nodes at $\mathrm{R}=0.375 \mathrm{~m}$ within the fracture zone $(\mathrm{Z}=0 \mathrm{~m})$ and at the top of the domain $(\mathrm{Z}=1.125 \mathrm{~m})$. 


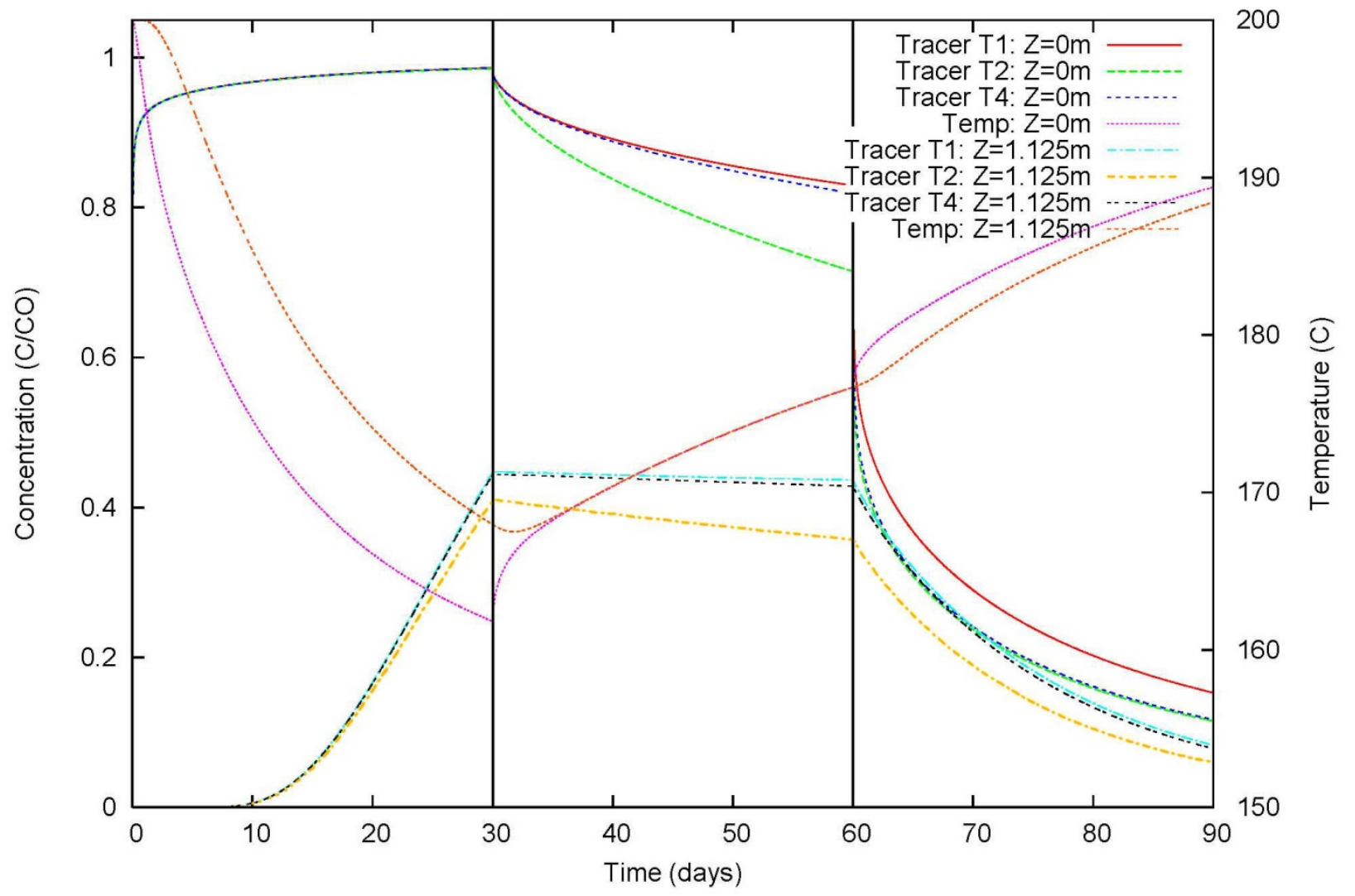

Figure 3.9. Simulated tracers and temperatures for an injection/shut-in/withdrawal test. Results are for nodes at $\mathrm{R}=2.625 \mathrm{~m}$ within the fracture zone $(\mathrm{Z}=0 \mathrm{~m})$ and at the top of the domain $(\mathrm{Z}=1.125 \mathrm{~m})$. 


\subsection{Semi-Analytical Model for Interwell Tracer Tests}

The semi-analytical model for the interwell tracer tests is described first below, followed by sections describing the evaluation of thermally degrading tracers to predict thermal breakthrough, conservative tracers with different diffusion coefficients to estimate fracture apertures or SA/V ratios, adsorbing tracers to estimate fracture apertures or SA/V ratios, and the combined use of tracers in interwell tracer tests to estimate flowing fracture spacings.

\subsection{Model Description}

A semi-analytical model was developed to conduct rapid scoping calculations of responses of diffusing, desorbing, and thermally degrading tracers in interwell tracer tests at EGS sites. The model is based on the RELAP computer model for solute transport in dual-porosity media (Reimus et al. 2003). The RELAP model combines the Laplace-domain dual-porosity transport equations derived by Maloszewski and Zuber (1984, Appendix; 1985) with Laplace-domain transfer functions that describe a finite-pulse injection, wellbore mixing, and recirculation (although the latter two features were not used here). The model can also simulate linear, reversible sorption (either rate-limited or equilibrium). Maloszewski and Zuber (1984, Appendix; 1985) assumed that tracer transport in fractures was described by the 1D advection-dispersion equation with 1D diffusion occurring into the surrounding matrix perpendicular to the flow direction in fractures. The model assumes 1) parallel-plate fractures of constant aperture, $2 \mathrm{~b}$, and either constant spacing, $L$, or infinite spacing (a semi-infinite matrix); 2 ) no concentration gradients across the fracture aperture; and 3) a steady flow rate in fractures. The most restrictive of these assumptions for EGS sites is the steady flow-rate assumption, which makes it impossible to simulate single-well tracer tests involving flow reversals. The assumed model geometry and the key governing equations, described in more detail below, are summarized in Figure 4.1.

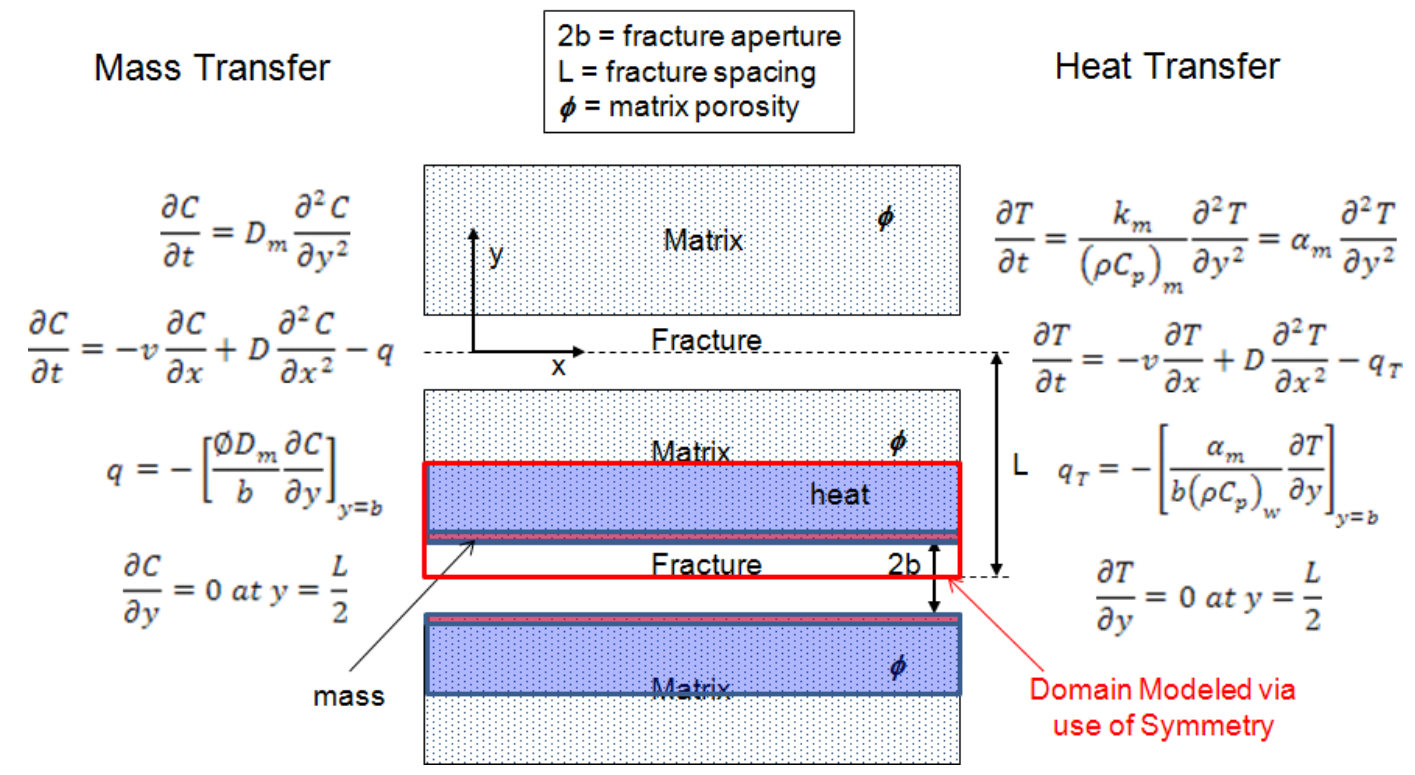

Figure 4.1. Semi-analytical model geometry and key governing mass (non-reactive) and heat transport equations (see text for more detail). The blue and red regions schematically show the matrix volumes affected by heat and mass transport, respectively, with heat transfer affecting a larger volume because it is much more rapid than mass transport. 
The equations describing dual-porosity transport of a non-reactive tracer under the conditions described above are (based on Maloszewski and Zuber [1984, Appendix]) as follows:

Fracture:

$$
\frac{\partial C}{\partial t}+V \frac{\partial C}{\partial x}-D \frac{\partial^{2} C}{\partial x^{2}}-\left.\frac{\phi_{m}}{\eta b} D_{m} \frac{\partial C_{m}}{\partial y}\right|_{y=b}=0
$$

Matrix:

$$
\frac{\partial C_{m}}{\partial t}-D_{m} \frac{\partial^{2} C_{m}}{\partial y^{2}}=0
$$

where $\quad C=$ solute concentration in fractures or flowing porosity, $\mathrm{g} / \mathrm{cm}^{3}$

$\mathrm{Cm}=$ solute concentration in matrix or nonflowing porosity, $\mathrm{g} / \mathrm{cm}^{3}$

$V=$ mean water velocity in fractures, $\mathrm{cm} / \mathrm{s}$

$D=$ solute dispersion coefficient in fractures, $\mathrm{cm}^{2} / \mathrm{s}$

$\phi m=$ matrix porosity

$b=$ fracture half-aperture, $\mathrm{cm}$ (fractures assumed to have an internal porosity of 1.0)

$\eta=$ internal porosity within fractures

$D m=$ solute diffusion coefficient in matrix, $\mathrm{cm}^{2} / \mathrm{s}$

$x=$ coordinate in flow direction

$y=$ coordinate in diffusion direction

$t=$ time, s.

A no-flux boundary condition is imposed at the centerline of each matrix block $\left(\left.\frac{\partial C_{m}}{\partial y}\right|_{y=\frac{L}{2}}=0\right)$ in recognition of the fact that the concentrations gradients from neighboring parallel-plate fractures will be equal and opposite at the midpoint distance between the fractures in the rectangular matrix block.

Additional terms are required in Equations (4.1) and (4.2) to describe reactive tracer transport. For a thermally degrading tracer, the term $k C$ must be added to left side of Equation (4.1), and the term $k C_{m}$ must be added to the left side of Equation (4.2), where $k$ is the decay constant given by Equation (2.1). For an adsorbing tracer, only linear sorption isotherms can be accommodated when using a semianalytical model, but adsorption and desorption rates can be specified as either fast or slow relative to transport velocities in the fractures. If the rates are sufficiently fast that local equilibrium partitioning between the solution and solid phases can be assumed, a simple retardation factor can be used to describe reactive transport in both the fractures and matrix. Equations (4.1) and (4.2) for such a rapidly sorbing tracer that also experiences thermal decay are as follows:

Fracture:

$$
R_{f} \frac{\partial C}{\partial t}+V \frac{\partial C}{\partial x}-D \frac{\partial^{2} C}{\partial x^{2}}+k C-\left.\frac{\phi_{m}}{\eta b} D_{m} \frac{\partial C_{m}}{\partial y}\right|_{y=b}=0
$$


Matrix:

$$
R_{m} \frac{\partial C_{m}}{\partial t}-D_{m} \frac{\partial^{2} C_{m}}{\partial y^{2}}+k C_{m}=0
$$

where $\quad R_{f}=$ retardation factor in fractures $=1+\frac{K_{a}}{b}$ for an open (unfilled) fracture or

$$
1+\frac{K_{a}}{b}+\frac{\rho_{B \eta} K_{d}}{\eta} \text { for a partially filled fracture }
$$

$R_{m}=$ retardation factor in matrix $=1+\frac{\rho_{B} K_{d}}{\phi_{m}}$

$k=$ thermal decay rate constant (1/s), as given by Equation (2.1)

$K_{a}=$ surface-area-based sorption partition coefficient, $\mathrm{cm}^{3} / \mathrm{cm}^{2}$

$K_{d}=$ mass-based sorption partition coefficient, $\mathrm{cm}^{3} / \mathrm{g}$

$\rho_{B}=$ bulk density of matrix $=\rho_{R}\left(1-\phi_{\mathrm{m}}\right)$, where $\rho_{R}=$ density of rock, $\mathrm{g} / \mathrm{cm}^{3}$

$\rho_{B \eta}=$ bulk density of fracture fill $=\rho_{R \eta}(1-\eta)$, where $\rho_{R \eta}=$ density of fill rock, $\mathrm{g} / \mathrm{cm}^{3}$.

If tracer adsorption and desorption are rate-limited (i.e., too slow for the local equilibrium assumption to apply), mass balance equations must also be written for immobile surfaces in the fractures and matrix. However, the Laplace-transformed equations for mobile and immobilized mass can be combined into a single Laplace-domain equation that can still be readily solved. The resulting Laplace-domain equations for the fractures and matrix in the case of a rate-limited (but still linear) reversibly sorbing tracer are as follows:

Fracture:

$$
\left(1+\frac{\frac{\rho_{B \eta}}{\eta} k_{f f}}{\frac{\rho_{B \eta}}{\eta} s+k_{f r}}\right) s \bar{C}+V \frac{\partial \bar{C}}{\partial x}-D \frac{\partial^{2} \bar{C}}{\partial x^{2}}+k \bar{C}-\left.\frac{\phi_{m}}{\eta b} D_{m} \frac{\partial \bar{C}_{m}}{\partial y}\right|_{y=b}=0
$$

Matrix:

$$
\left(1+\frac{\frac{\rho_{B}}{\phi_{m}} k_{m f}}{\frac{\rho_{B}}{\phi_{m}} s+k_{m r}}\right) s \bar{C}_{m}-D_{m} \frac{\partial^{2} \bar{C}_{m}}{\partial y^{2}}+k \bar{C}_{m}=0
$$

where $\quad k_{f f}=$ sorption rate constant in fracture, $\mathrm{cm}^{3} / \mathrm{cm}^{2}-\mathrm{s}$

$k_{f r}=$ desorption rate constant in fracture, $1 / \mathrm{s}$

$k_{m f}=$ sorption rate constant in matrix, $\mathrm{cm}^{3} / \mathrm{g}-\mathrm{s}$

$k_{m r}=$ desorption rate constant in matrix, $1 / \mathrm{s}$

$s=$ Laplace transformation variable

$\bar{C}=$ Laplace transformed concentration (either fracture or matrix). 
Note that the expressions in parentheses at the beginning of Equations (4.5) and (4.6) are replaced by the retardation factors, $R_{f}=1+\frac{\rho_{B \eta}}{\eta} \frac{k_{f f}}{k_{f r}}=1+\frac{\rho_{B \eta}}{\eta} K_{a}$, and $R_{m}=1+\frac{\rho_{B}}{\phi_{m}} \frac{k_{m f}}{k_{m r}}=1+\frac{\rho_{B}}{\phi_{m}} K_{d}$, respectively, in the case of rapid sorption and desorption.

The Laplace-domain solution of Equations (4.5) and (4.6) and the subsequent inversion of the result to the time domain are described by Reimus et al. (2003, Appendix A). Note that Equations (4.5) and (4.6) reduce to a single-porosity system if the matrix porosity, $\phi_{m}$, or the matrix diffusion coefficient, $D_{m}$, are set equal to zero. The semi-analytical model also allows for easy simulation of injection functions, well-mixed boreholes, pipe delays, and importantly for EGS sites, recirculation of tracer mass into the injection well. The convolution of these processes is accomplished by a simple multiplication of Laplace transfer functions in the Laplace domain:

$$
C(t)=\int_{0}^{\mathrm{t}} A(\tau) \cdot B(t-\tau) d \tau \Rightarrow \bar{C}(s)=\bar{A}(s) \cdot \bar{B}(s)
$$

where the time-domain integral is a classic convolution integral, $A, B$, and $C$ are time domain functions, and $\bar{A}(s), \bar{B}(s)$, and $\bar{C}(s)$ are the Laplace transforms of these functions.

The transfer functions used in the RELAP model for injection functions, well-mixed boreholes, pipe delays, and tracer recirculation are as follows:

Injection pulse:

$$
\bar{I}(s)=C_{o}\left(\frac{1-\exp \left(-T_{p} s\right)}{s}\right)
$$

where $C_{o}$ is concentration of injection pulse, $\mathrm{g} / \mathrm{cm}^{3}$, and $T_{p}$ is the duration of injection pulse, $\mathrm{s}$ or hr.

Well-mixed borehole:

$$
\bar{W}(s)=\frac{\gamma}{\gamma+\mathrm{s}}
$$

where $\gamma$ is the time constant, generally the volumetric flow rate divided by the volume of well-mixed region, $1 / \mathrm{s}$ or $1 / \mathrm{hr}$.

Pipe delay:

$$
\bar{P}(s)=\exp \left(-T_{d} s\right)
$$

where $T_{d}$ is the delay time in pipe, s or hr. 
Tracer recirculation:

$$
\bar{F}_{R}(s)=\frac{\bar{F}(s)}{1-\varepsilon \bar{F}(s)}
$$

where $\bar{F}_{R}(s)$ is the transfer function with recirculation, $\bar{F}(s)$ is the transfer function without recirculation, and $\varepsilon$ is the recirculation ratio $(0=$ no recirculation, $1=$ full recirculation $)$.

The ability to simulate heat transfer in an interwell flow system having the geometry of Figure 4.1 was incorporated into RELAP by taking advantage of the analogies between heat and mass transport. Equations (4.1) and (4.2) for heat transfer in a dual-porosity system are as follows:

Fracture:

$$
\left(\rho C_{p}\right)_{w}\left(\frac{\partial T}{\partial t}+V \frac{\partial T}{\partial x}-D \frac{\partial^{2} T}{\partial x^{2}}\right)-\left.\frac{k_{m}}{b} \frac{\partial T_{m}}{\partial y}\right|_{y=b}=0
$$

Matrix:

$$
\left(\rho C_{p}\right)_{m} \frac{\partial T_{m}}{\partial t}-k_{m} \frac{\partial^{2} T_{m}}{\partial y^{2}}=0
$$

where $\quad T=$ temperature in fractures or flowing porosity, $\mathrm{K}$

$T_{m}=$ temperature in matrix, $\mathrm{K}$

$\rho=$ density (water or matrix), $\mathrm{g} / \mathrm{cm}^{3}$ (subscript $w$ for water, $m$ for matrix)

$C_{p}=$ heat capacity (water or matrix), cal $/ \mathrm{g}-\mathrm{K}$ (subscript $w$ for water, $m$ for matrix)

$k_{m}=$ thermal conductivity of matrix, cal $/ \mathrm{cm}-\mathrm{s}-\mathrm{K}$.

Similar to the mass transfer equations, a no-flux boundary condition is imposed for heat transfer at the centerline of each matrix block $\left(\left.\frac{\partial T}{\partial y}\right|_{y=\frac{L}{2}}=0\right)$ in recognition that the temperature gradients from

neighboring parallel-plate fractures are equal and opposite at the midpoint distance between the fractures.

Note that in Equations (4.12) and (4.13), it is understood that the matrix values of $\rho, C_{p}$, and $k_{m}$ will depend on the matrix porosity; i.e., these values can be approximated from $X_{m}=\phi X_{w}+(1-\phi) X_{R}$, where $X$ is a general property $\left(\rho, C_{p}\right.$, or $\left.k_{m}\right)$, and $X_{R}$ denotes the value of the property for the rock, while $X_{w}$ is the value of the property for water.

The analogies between Equations (4.12) and (4.13) and Equations (4.1) and (4.2) are obvious, and a solution for temperature in Equations (4.12) and (4.13) can be readily obtained from a solution for 
concentration in Equations (4.1) and (4.2) by simply substituting $k_{m} /\left(\rho C_{p}\right)_{w}$ for $\phi D_{m}$ in Equation (4.1) and $k_{m} /\left(\rho C_{p}\right)_{m}$ for $D_{m}$ in Equation (4.2). This is what was done to obtain a temperature solution using the existing RELAP concentration solution.

RELAP is not capable of providing a true simultaneous solution of temperature and concentration because the Laplace-domain algorithm only solves for one variable at a time while all other variables are held constant. However, because heat transfer in an EGS has essentially no dependence on solution concentrations, it is acceptable to first obtain a temperature solution and then obtain a concentration solution that includes the temperature dependence of tracer thermal decay. The Laplace-domain algorithm also provides a temperature or concentration solution only at the exit of the simulated domain, so spatial variability in temperature must be obtained by dividing the domain into discrete segments, each of which has a separate temperature solution. A consistent overall temperature solution is obtained by linking the segments together by convolution (e.g., Figure 4.2), which is achieved in the Laplace domain via Equation (4.7). Once a spatial temperature profile is obtained (at a given point in time), a tracer transport solution can be obtained by similarly linking together segments and using the temperature calculated for each segment to determine the temperature-dependent decay rate constant in each segment.

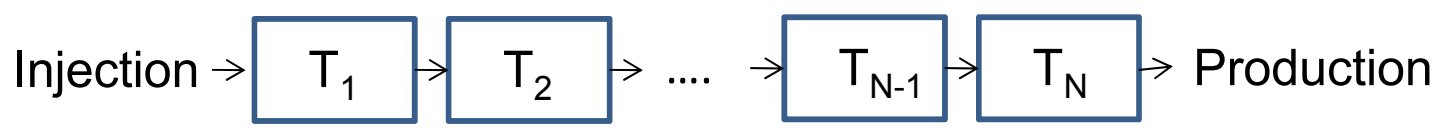

Figure 4.2. Convolution in the Laplace domain of several model segments (each equivalent to the model domain of Figure 4.1) to obtain a spatially discretized temperature solution as a function of time between an injection and production well.

This approach involves the following assumptions:

- The temperature within each segment is assumed to be constant (equal to the exit temperature of the segment). The accuracy associated with this assumption can be tested by simply specifying more segments and comparing the results for different spatial discretizations.

- The calculated temperatures are strictly only for the fractures, not for the matrix where there will be a temperature gradient. However, this assumption is not very restrictive because mass transfer is much slower than heat transfer, so the matrix temperature very near the fractures (as far as tracers will diffuse) will be essentially the same as the temperature of the fractures. Note that values of $k_{m} /\left(\rho C_{p}\right)_{m}$ are typically 3 to 5 orders of magnitude greater than values of $D_{m}$, meaning that heat will penetrate 1.5 to 2.5 orders of magnitude farther into the matrix than tracers at any point in time.

- The temperatures for any given tracer simulation are assumed to be constant in each segment for the duration of the simulated tracer test. In reality, the temperatures in the segments will continually decrease with time, so this assumption will be most valid when tracer travel times are relatively short compared to the time scales over which temperatures change. The error introduced by this assumption must be evaluated for each system simulated. If tracer test durations are quite long, it would be advisable to calculate temperatures at approximately the midpoint of the tracer test rather than at the start or end of the tracer test. 
It is also important to account for the temperature dependence of matrix diffusion coefficients in geothermal systems, which can be significant because temperatures may vary by as much as $200^{\circ} \mathrm{C}$ between injection and production wells. Diffusion coefficients vary with temperature according to the following:

$$
\frac{D_{T_{2}}}{D_{T_{1}}}=\frac{T_{2}}{T_{1}} \frac{\mu_{T_{1}}}{\mu_{T_{2}}}
$$

where $D=$ diffusion coefficient $\left(\mathrm{cm}^{2} / \mathrm{s}\right), T=$ temperature $(\mathrm{K}), \mu=$ water viscosity $(\mathrm{g} / \mathrm{cm}-\mathrm{s})$, and the subscripts $T_{1}$ and $T_{2}$ refer to two different temperatures. The temperature dependence of the viscosity of water can be approximated by (Likhachev 2003) the following equation:

$$
\frac{\mu_{T_{1}}}{\mu_{T_{2}}}=\frac{10^{\frac{247.8}{\left(T_{1}-240\right)}}}{10^{\frac{247.8}{\left(T_{2}-240\right)}}}
$$

Combining Equations (4.14) and (4.15), and using $25^{\circ} \mathrm{C}$ as a reference temperature, the following expression is obtained for diffusion coefficients (or matrix diffusion coefficients) as a function of temperature:

$$
D_{T}=D_{25 C} \frac{(T+273)}{298}\left(\frac{37.013}{10^{\frac{247.8}{(T+133)}}}\right)
$$

Equation (4.16) was used in the RELAP heat and mass transport model to account for the temperature dependence of diffusion coefficients.

\subsection{Evaluation of the Capability of Thermally Degrading Tracers to Predict Thermal Breakthrough}

The RELAP combined mass and heat transport model was used to evaluate the ability to estimate when thermal breakthrough would occur in a multi-well EGS configuration using thermally degrading tracers. It also allowed an evaluation of the range of values of Arrhenius parameters, $A$ and $E_{a}$, that are required to obtain interpretable responses of thermally degrading tracers.

\subsubsection{Thermal Breakthrough Concepts and Parameters}

Before describing this work, it is useful to review the concepts of thermal breakthrough and the parameters that dictate when it will occur. Thermal breakthrough is loosely defined as the onset of cooler temperatures at a production well in a geothermal reservoir as a result of extracting heat from the reservoir rock by injection and circulation of cool water. The thermal breakthrough time is somewhat subjective in that it depends on the temperature drop that is used to define thermal breakthrough. For example, the breakthrough time will be much shorter if a temperature drop of $1 \%$ is considered thermal 
breakthrough than if a temperature drop of $5 \%$ or $10 \%$ is considered thermal breakthrough. However, regardless of the definition, the thermal breakthrough time will have the following dependence on average fracture apertures $(2 b)$ and fracture spacings $(L)$ in a geothermal reservoir:

$$
\tau_{\text {thermal }} \propto \frac{b\left(\eta \rho_{w} C p_{w}+(1-\eta) \rho_{R} C p_{R}\right)+L / 2\left(\phi_{m} \rho_{w} C p_{w}+\left(1-\phi_{m}\right) \rho_{R} C p_{R}\right)}{b \eta \rho_{w} C p_{w}}
$$

where $\propto$ indicates a proportionality and all variables are as defined above. Equation (4.17) is technically the definition of a thermal retardation factor, or the ratio of the arrival time of cool water to the arrival time of a conservative tracer in a geothermal system. Note that if the fractures contain no fill material (i.e., $\eta=0$ ), then Equation (4.14) becomes

$$
\tau_{\text {thermal }} \propto \frac{b \rho_{w} C p_{w}+L / 2\left(\phi_{m} \rho_{w} C p_{w}+\left(1-\phi_{m}\right) \rho_{R} C p_{R}\right)}{b \rho_{w} C p_{w}}
$$

The first term in the numerator of both Equations (4.17) and (4.18) is generally negligible compared to the second term. Several RELAP simulations confirmed that this relationship holds for a very wide range of fracture apertures and spacings. However, as fracture spacings become extremely large (with the ultimate being a semi-infinite matrix), Equations (4.17) and (4.18) predict that thermal breakthrough will never occur, which is unrealistic.

Figure 4.3 shows temperatures as a function of time, as determined using the RELAP heat and mass transfer model, in a multi-well EGS system with the model parameters of Table 4.1. These parameters correspond to a system that will exhibit thermal drawdown rather quickly, primarily because of the small fracture spacing. The different curves in Figure 4.3 correspond to different fractional distances between the injection and production wells, with a value of 1 corresponding to the temperature history at the production well.

Table 4.1. Model parameters used in RELAP simulations of Figure 4.3 and Figure 4.4.

\begin{tabular}{ll}
\hline Initial temperature of reservoir & $250^{\circ} \mathrm{C}$ \\
Injection water temperature & $150^{\circ} \mathrm{C}$ \\
Mean water residence time in reservoir & $300 \mathrm{hr}$ \\
$\begin{array}{l}\text { Peclet number (well separation divided by longitudinal } \\
\text { dispersivity) }\end{array}$ & 10 \\
Fracture half-apertures & $0.05 \mathrm{~cm}$ \\
Fracture spacing & $10 \mathrm{~cm}$ \\
Matrix porosity & $0.005(0.5 \%)$ \\
Tracer matrix diffusion coefficient & $3 \times 10^{-6} \mathrm{~cm}^{2} / \mathrm{s} \mathrm{at} 25^{\circ} \mathrm{C}$ \\
Arrhenius thermal decay parameters & $A=2.5 \times 10^{7} \mathrm{~s}^{-1}$ \\
& $E_{a}=32,000 \mathrm{kcal} / \mathrm{mol}$ \\
Rock and water properties (density and thermal) & Corresponding to basalt and dilute water \\
\hline
\end{tabular}




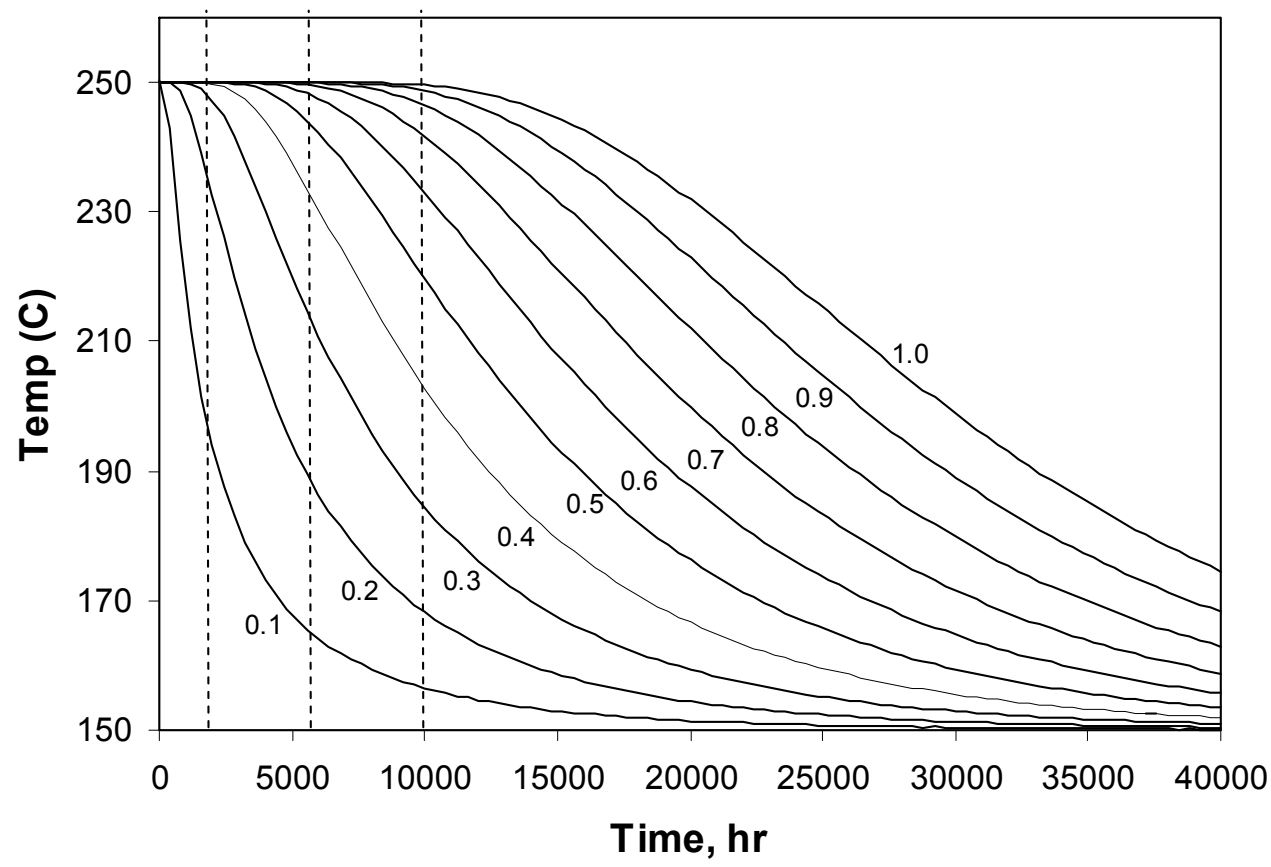

Figure 4.3. Simulated temperature as a function of time and fractional distance between the injection well and production well (see Table 4.1 for model parameters). The numbers next to the curves are the fractional distance between the wells, with 1.0 being the production well location. Dashed lines correspond to times when tracer tests were simulated (see Figure 4.4).

Figure 4.4 shows breakthrough curves of a conservative tracer compared to the breakthrough curves of a thermally degrading tracer at three different times after starting the injection of cool water into the reservoir. The Arrhenius decay parameters for the tracer were $E_{a}=32,000 \mathrm{cal} / \mathrm{mol}$ and $A=2.5 \times 10^{7} \mathrm{~s}^{-1}$. As Figure 4.3 shows, thermal breakthrough at the production well occurs at approximately 12,000 hr (with thermal breakthrough defined here as the onset of a temperature decrease). It is apparent that there is a significant difference in tracer breakthrough curves at 2000 and $6000 \mathrm{hr}$ after the start of operations, with the peak tracer concentration increasing by $\sim 40 \%$ in the later test because of lower temperatures experienced in the flow system. The peak concentration then increases by another $\sim 33 \%$ in the test at $10,000 \mathrm{hr}$, for which temperatures have decreased even further. These results show that thermally degrading tracers can qualitatively provide a good early indication of thermal breakthrough significantly before it actually occurs.

To explore the potential use of thermally decaying tracers further, the RELAP model was used to define ranges of values of the Arrhenius decay parameters $A$ and $E_{a}$ (see Equation (2.1)) that are necessary to obtain interpretable responses of thermally degrading tracers. If these parameter values are such that too much thermal decay occurs, then the tracers will never be detected at the production well. On the other hand, if too little decay occurs, the tracers will have the same responses as nondecaying conservative tracers. In either case, the information obtained from the tracer test will only be qualitative and cannot be used to estimate thermal breakthrough times. 


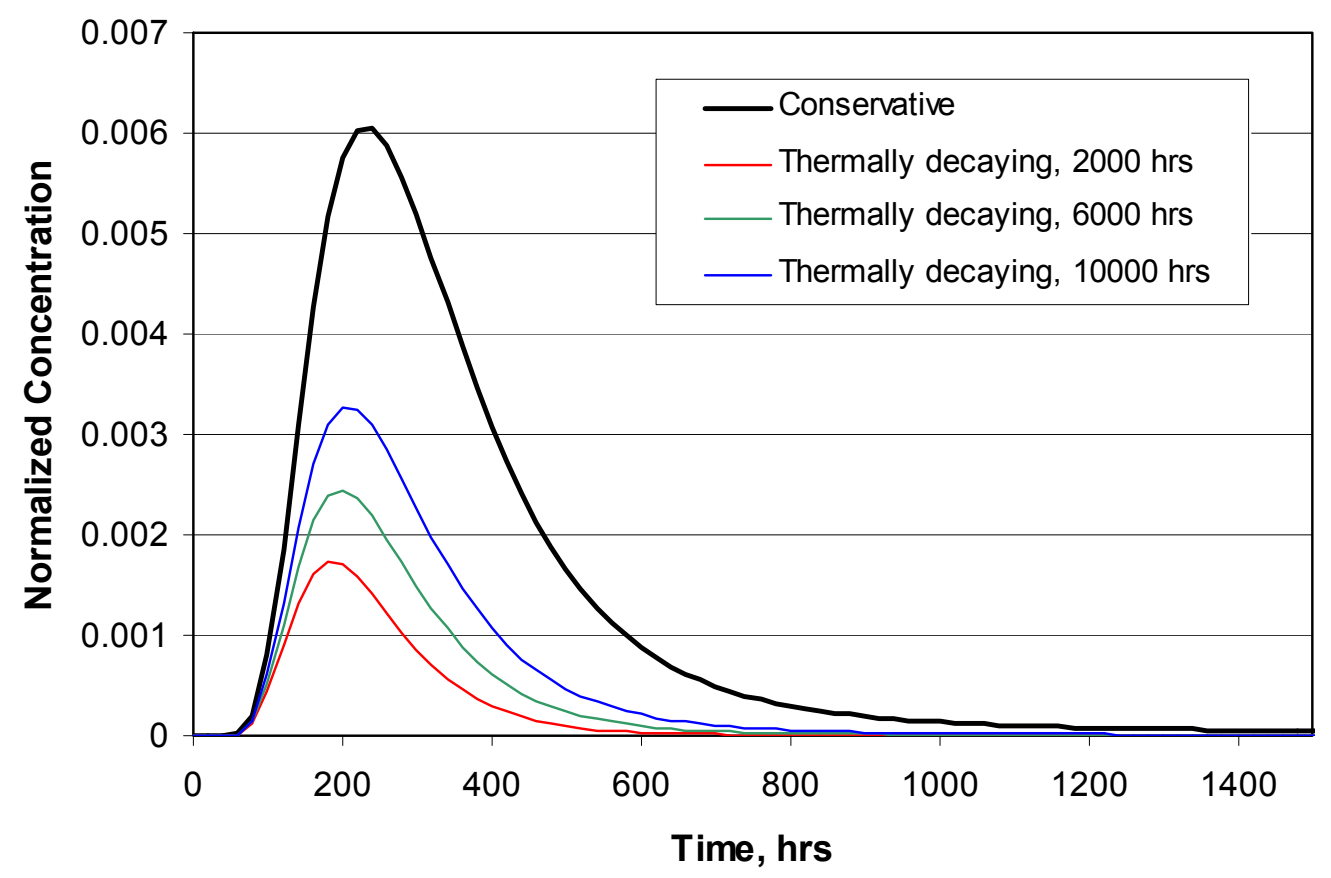

Figure 4.4. Simulated breakthrough curves of a conservative tracer and thermally degrading tracer at three different times after the start of reservoir operations. See Figure 4.3 for temperature profiles at each time.

Figure 4.5 shows ranges of values of $A$ and $E_{a}$, expressed as dimensionless parameters, for thermally degrading tracers that provide breakthrough curves that can be quantitatively interpreted. Example breakthrough curves associated with the two dashed lines representing the upper and lower bounds are shown to the right in Figure 4.5. The ranges of $A$ and $E_{a}$ are dependent on both the initial reservoir temperature and the mean tracer residence time in the reservoir.

Mathematically, the requirement for a readily interpretable tracer response is that the value of the decay rate constant, $\mathrm{k}$, fall within an approximate range from $0.3 / \tau$ to $10 / \tau$, where $\tau$ is the mean tracer residence time in the system (this is the range defined by the 2D parameter space between the dashed lines of Figure 4.5). These bounds for $\mathrm{k}$ can be extended more in the upward direction if a tracer has a very low detection limit and thus a large dynamic range of concentrations over which it can be quantified. The lower bound is fixed regardless of these considerations because analytical precision rather than the detection/quantification limit is the limiting factor at low rates of degradation (i.e., the key in this case is to be able to distinguish the tracer breakthrough curve from that of a conservative tracer rather than being able to quantify the tracer at low concentrations).

The RELAP model was next used to evaluate the sensitivity of thermally degrading tracer responses to spatial variability in fracture apertures and fracture spacings in interwell tracer tests in geothermal reservoirs. To simulate spatial variability, different fracture apertures or spacings were assigned to each model segment used to obtain a spatially discretized temperature solution. The average fracture apertures or spacings in each segment were randomly sampled from lognormal distributions with specified means and standard deviations (of log values). Separate cases were considered in which the residence time was assumed to be the same in each segment (i.e., linear flow) and in which the residence times were varied in 
each segment according to an overlapping radial flow dependence as a function of distance from the injection and production wells (biradial flow). In the latter case, the flow rates were fastest and the residence times were shortest in the segments near the wells, and they were slowest and longest, respectively, in the segments equidistant between the wells. This latter case is believed to be more representative of a two-well flow system in a geothermal reservoir, although under some conditions linear flow could be approximated. The overall residence time and Peclet number in each case, regardless of linear or biradial flow, was kept the same.
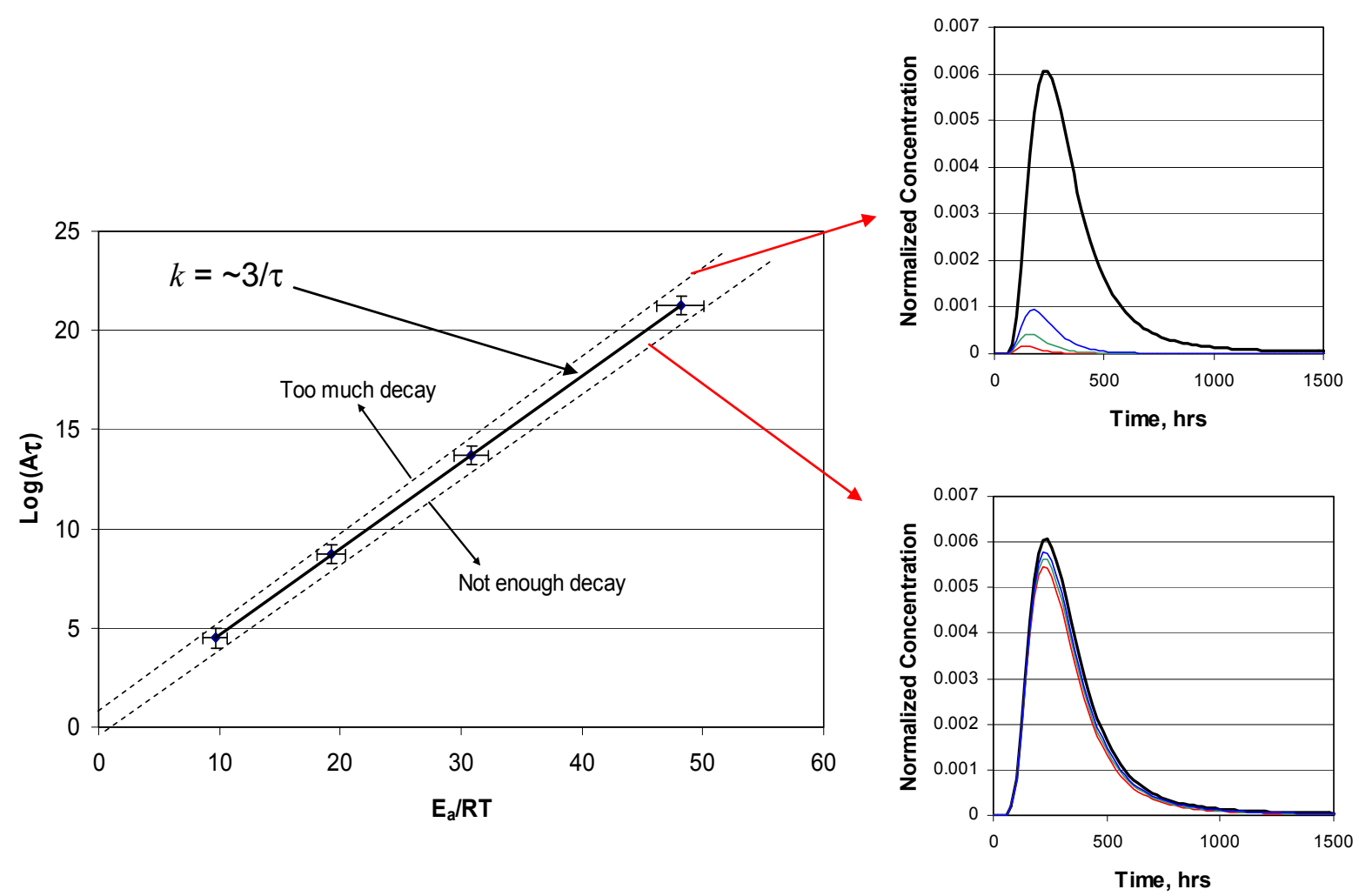

Figure 4.5. Ranges of Arrhenius decay parameters $A$ and $E_{a}$ (expressed as $\log (A \tau)$ and $E_{a} / \mathrm{RT}$ ) providing quantifiable interpretations of thermally degrading tracers relative to conservative tracers. Plots to the right show breakthrough curves associated with the dashed lines in the plot on the left. The different colored breakthrough curves have the same meanings as in Figure 4.2.

\subsubsection{Thermal Breakthrough Parameter Sensitivity Cases}

In the remainder of this section, RELAP simulations were conducted using 20 model segments with the baseline parameters of Table 4.2 (with variability between segments superimposed as described below). The properties of Table 4.2 are similar, but not the same, as those used to generate Figure 4.3 and Figure 4.4 (Table 4.1). Most notably, the fracture aperture and spacing were increased to be more representative of a real geothermal system, and the matrix porosity was increased by an order of magnitude to increase the importance of matrix diffusion. 
Table 4.2. Model parameters used in RELAP simulations with 20 model segments.

\begin{tabular}{ll}
\hline Initial temperature of reservoir & $250^{\circ} \mathrm{C}$ \\
Injection water temperature & $50^{\circ} \mathrm{C}$ \\
Mean water residence time in reservoir & $400 \mathrm{hr}$ \\
Peclet number & $10(12 \mathrm{in}$ case of biradial flow) \\
Fracture half-apertures & $0.25 \mathrm{~cm}$ \\
Fracture spacing & $200 \mathrm{~cm}$ \\
Matrix porosity & $0.05(5 \%)$ \\
Tracer matrix diffusion coefficient & $1 \times 10^{-6} \mathrm{~cm}^{2} / \mathrm{s}$ at $25^{\circ} \mathrm{C}$ \\
Arrhenius thermal decay parameters & $A=5 \times 10^{7} \mathrm{~s}^{-1}$ \\
& $E_{a}=32,000 \mathrm{kcal} / \mathrm{mol}$ \\
Rock and water properties (density and thermal) & Corresponding to basalt and dilute water \\
\hline
\end{tabular}

Figure 4.6 shows the results of RELAP simulations that illustrate the impact of accounting for the temperature dependence of matrix diffusion coefficients when using the parameters listed above (with no variations). The tracer breakthrough curves calculated assuming a temperature dependence have significantly lower peak concentrations and longer tails than the cases in which the matrix diffusion coefficient was assumed to be constant at its $25^{\circ} \mathrm{C}$ value because the higher temperatures result in greater matrix diffusion, which lowers peak concentrations and extends tails of breakthrough curves. Figure 4.6 also shows that the linear and biradial flow assumptions can produce the same conservative (nondecaying) tracer breakthrough curve, although there are some differences between the thermally degrading tracer breakthrough curves because of the slightly different temperature histories experienced by the tracers in the different flow regimes. The Peclet number for the biradial flow cases had to be increased from 10 to 12 to produce the same conservative tracer breakthrough curve as the linear flow cases, which indicates that a biradial flow field has inherently greater underlying dispersion than a linear flow field.

Figure 4.7 shows the results of 10 RELAP simulations of tracer tests in which a conservative tracer and a thermally degrading tracer were assumed to be simultaneously injected. The model parameters in these simulations were kept constant in each segment (Table 4.2) except that the fracture half-apertures were allowed to vary from segment to segment with a standard deviation of $0.3 \log$ units. The tracer tests were simulated as taking place $10,000 \mathrm{hr}$ after the start of injection of cool water, so the reservoir was partially cooled at the time of the tracer test. However, thermal breakthrough did not occur in the simulations until approximately $40,000 \mathrm{hr}$ after the start of cool water injection (with some variability). Five of the 10 simulations were conducted assuming linear flow and 5 were conducted with the same aperture values but assuming biradial flow. The similarity of non-decaying tracer breakthrough curves in Figure 4.7 reflects the similarity of the time-weighted averages of the half-aperture values in each of the simulations; it is known (Deng et al. 2010) that matrix diffusion rates are inversely proportional to the time-weighted averages of half-aperture values, and apparently the temperatures in the fractures were also similar enough that there was little difference in average matrix diffusion rates as a result of the minor differences in temperatures in each simulation. The thermally degrading tracer breakthrough curves in Figure 4.7 show slightly more variability than the non-decaying tracer curves of Figure 4.7. This 
variability reflects the greater temperature dependence of the tracer thermal degradation rate than the temperature dependence of diffusion coefficients.
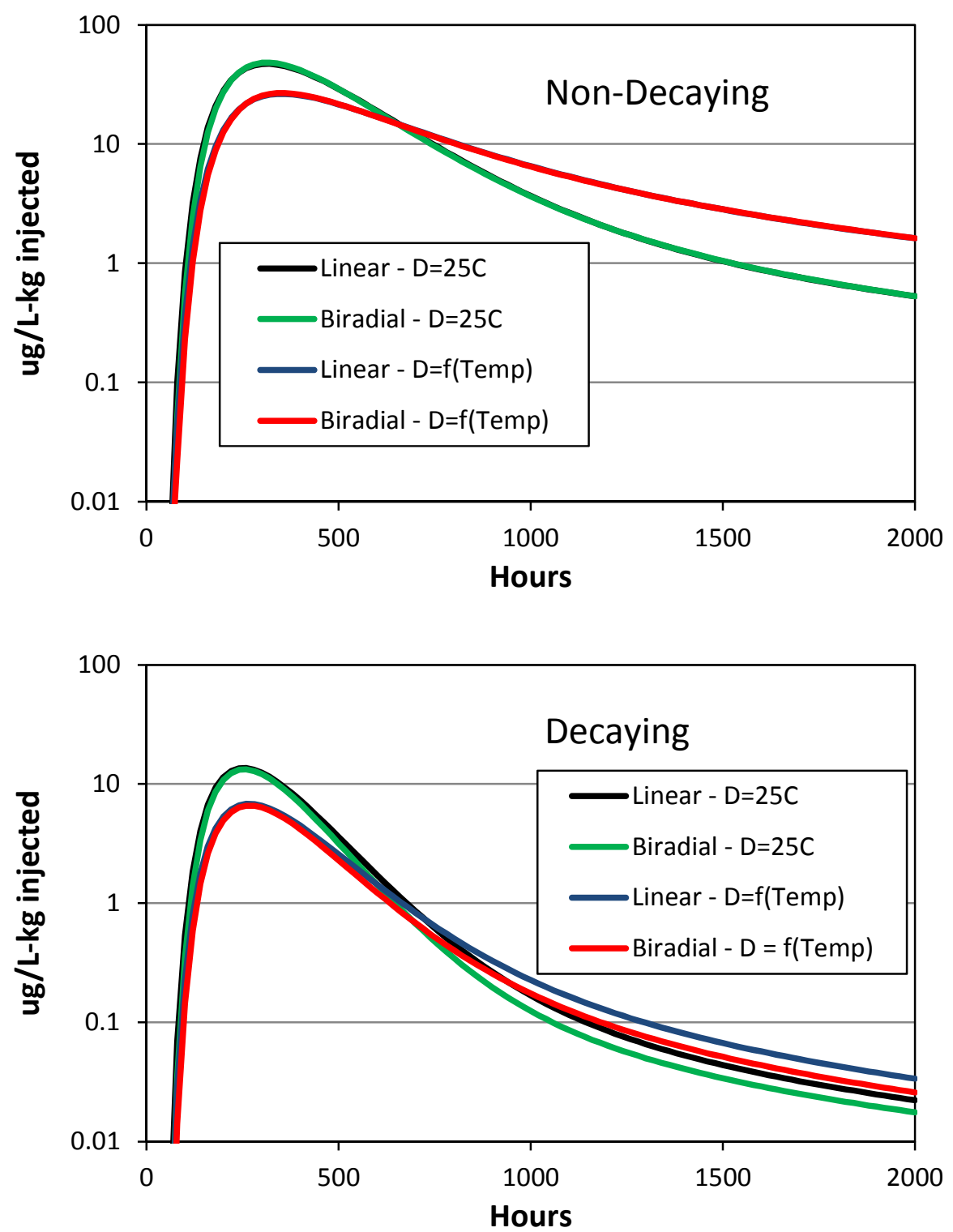

Figure 4.6. Breakthrough curves of conservative non-decaying (top) and thermally degrading (bottom) tracers under linear and biradial flow conditions using the model parameters listed in Table 4.2. Note the differences in the breakthrough curves when the temperature dependence of the matrix diffusion coefficient is accounted for instead of assuming the matrix diffusion coefficient at $25^{\circ} \mathrm{C}$. Note also that although there are no differences in the non-decaying breakthrough curves for linear and biradial flow, there are slight differences in thermally degrading tracer breakthrough curves for these two flow conditions. 


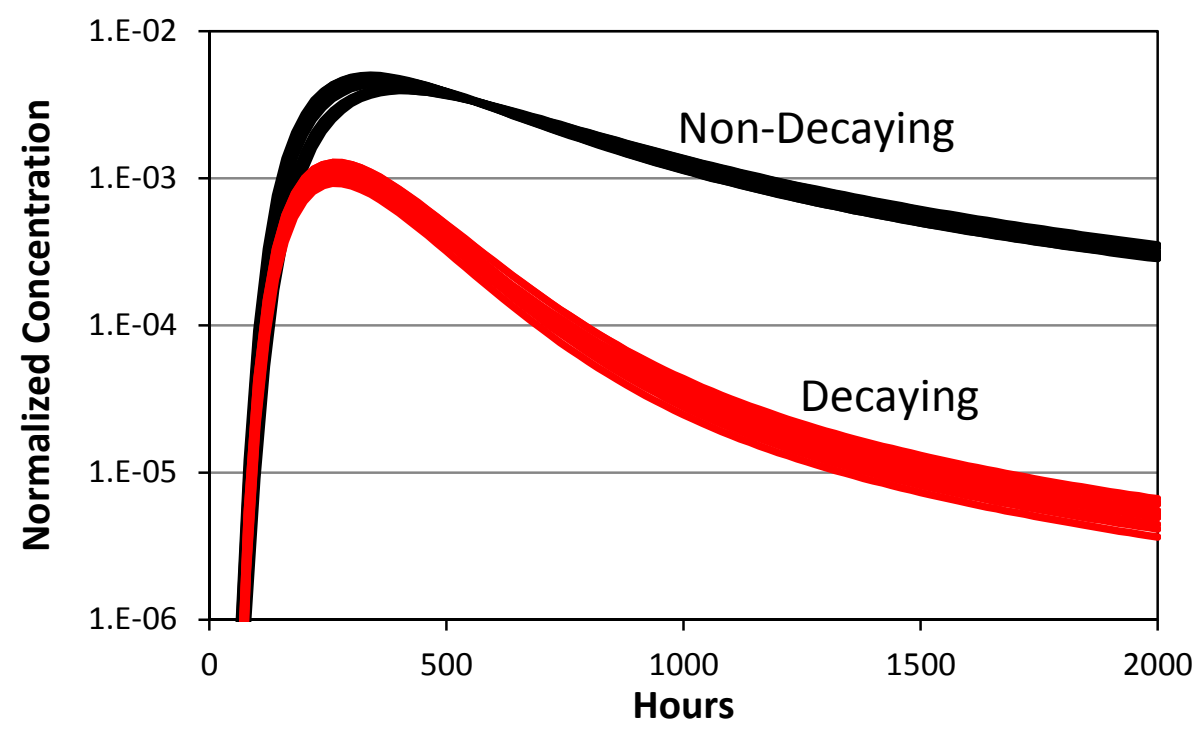

Figure 4.7. Breakthrough curves of conservative non-decaying (black) and thermally degrading (red) tracers from 10 simulations having 20 segments with a standard deviation of log apertures of $0.3 \log$ units. Five of the simulations assumed linear flow and five assumed biradial flow.

Figure 4.8 shows the results of 10 RELAP simulations in which all model parameters were kept constant in each segment (Table 4.2) except that the fracture spacings were allowed to vary from segment to segment with a standard deviation of $0.5 \log$ units. As with Figure 4.7, the tracer tests were simulated as taking place $10,000 \mathrm{hr}$ after the start of injection of cool water, with thermal breakthrough occurring approximately $40,000 \mathrm{hr}$ after the start of cool water injection. The results are qualitatively similar to those of Figure 4.7 but there is less variability in the conservative tracer responses and somewhat more variability in the thermally degrading tracer responses. The thermally degrading tracer breakthrough curves have more variability presumably because of the slightly larger standard deviation of the fracture spacings compared to the standard deviation of the fracture apertures in Figure 4.7, which causes more variability in the thermal drawdown in the system at the time of the tracer test $(10,000 \mathrm{hr})$ and hence more variability in the amount of thermal decay. The non-decaying tracer breakthrough curves have very little variability because their differences are much more dependent on fracture apertures than fracture spacings, and the apertures in this case were held constant.

In each of the simulations of Figure 4.7 and Figure 4.8, the time of thermal breakthrough was noted, where thermal breakthrough was defined as the time at which a temperature drop occurred at the production well equal to $5 \%$ of the difference between the injection water temperature and the initial reservoir temperature. Figure 4.9 shows the thermal breakthrough times in all the simulations of Figure 4.7 and Figure 4.8 as a function of the time-weighted value of $L / 2 b$, which is directly proportional to the right-hand side of Equation (4.18), the thermal retardation factor for an open fracture. The data for the simulations of Figure 4.7 and Figure 4.8 plot as two separate lines. The reason for this separation was determined to be a greater dispersion of heat between the injection and production well in the simulated cases of Figure 4.8 relative to Figure 4.7 (i.e., a shallower temperature front for Figure 4.8 than for Figure 4.7). For the same value of time-weighted $L / 2 b$, this greater dispersion caused an earlier temperature drop at the production well in the cases of Figure 4.8 than in the cases of Figure 4.7. Apparently, variability in fracture spacing causes more heat dispersion than variability in aperture, 
although this difference might also be a consequence of the smaller standard deviations of apertures than fracture spacings in the two sets of simulations (this possibility was not evaluated). It was verified that the time at which a 50\% drop in temperature occurred at the production well (approximately the mean temperature arrival time) was about the same in the cases of Figure 4.7 and Figure 4.8. That is, if thermal breakthrough was defined as a 50\% temperature drop, the cases of Figure 4.7 and Figure 4.8 would plot on top of each other in Figure 4.9, which confirms that the earlier thermal breakthrough for the cases of Figure 4.8 is a consequence of greater heat dispersion, not a fundamental difference in the retardation of temperature/heat.

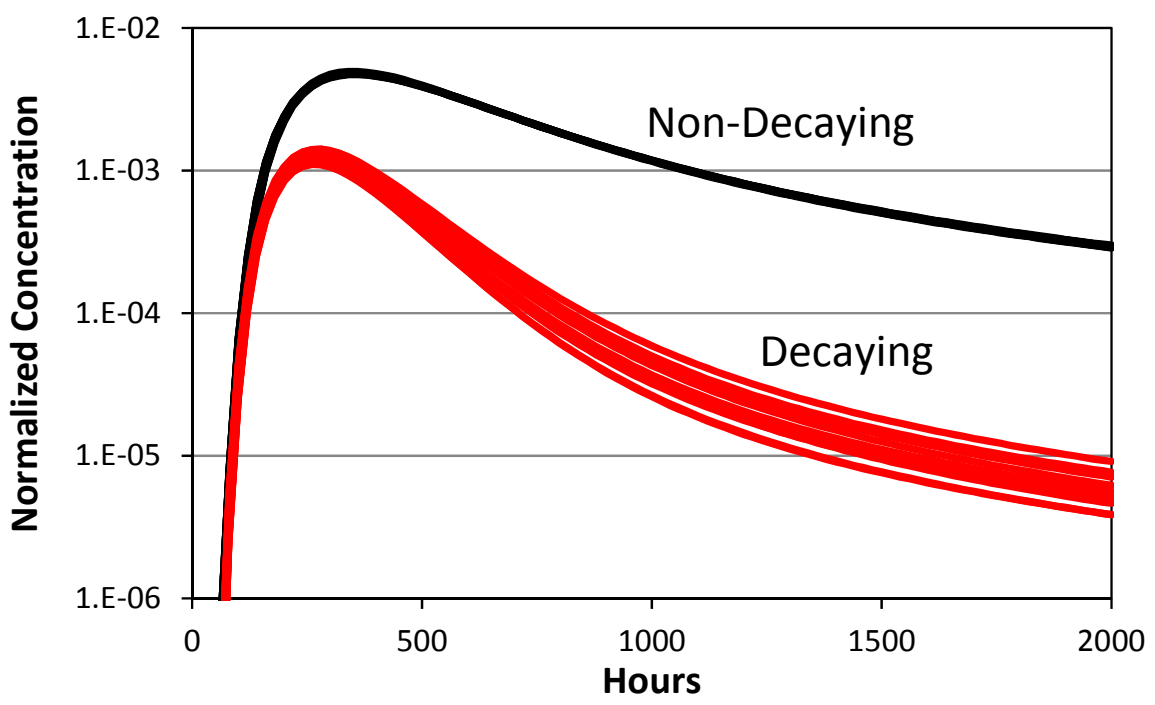

Figure 4.8. Breakthrough curves of conservative non-decaying (black) and thermally degrading (red) tracers from 10 simulations having 20 segments with a standard deviation of $\log$ fracture spacing of $0.5 \log$ units. Five of the simulations assumed linear flow and five assumed biradial flow.

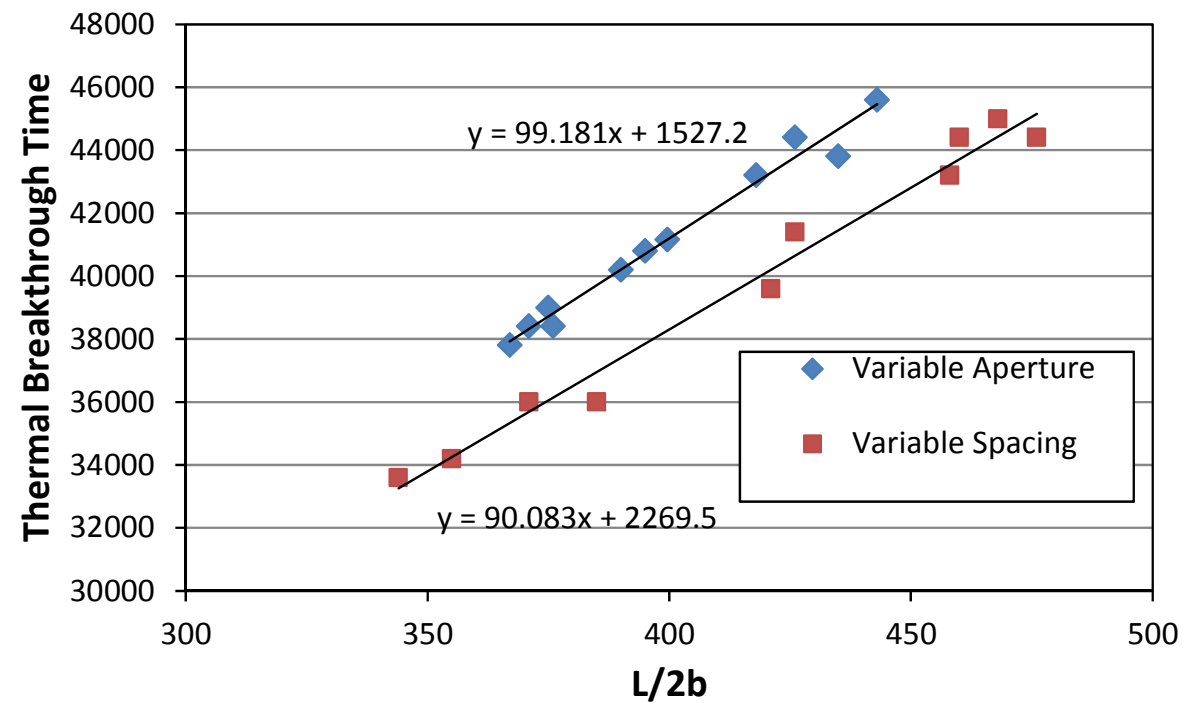

Figure 4.9. Thermal breakthrough times as a function of $L / 2 b$ for simulations in which fracture apertures were varied by $0.3 \log$ units or fracture spacings were varied by $0.5 \log$ units. 
It would be of great practical interest to devise a strategy for using thermally degrading tracers in conjunction with conservative tracers in the manner of Figure 4.4 to predict when thermal breakthrough might occur in a geothermal system. To explore this possibility, each of the cases of Figure 4.8 was repeated but with tracer tests were simulated at $0 \mathrm{hr}$ and 25,000 hr (instead of 10,000 hr) after the start of cool water injection and then another tracer test simulated at the time of thermal breakthrough.

Figure 4.10 shows the thermally decaying tracer breakthrough curves corresponding to each of the tracer test times and a single conservative tracer breakthrough curve (identical for all test times) for the two cases of Figure 4.8 that had the fastest and slowest thermal breakthrough times. It is apparent that the separation between the breakthrough curves for the fast thermal breakthrough is much less than that for the slow thermal breakthrough. It is also apparent that at the time of thermal breakthrough, the peak concentration of the thermally degrading tracer is approximately the same as the peak concentration of the conservative tracer (although this result is a function of both the definition of thermal breakthrough and the Arrhenius decay parameters). Of course, it is not practical to conduct a tracer test after thermal breakthrough occurs, so the tests of greatest interest from the standpoint of predicting thermal breakthrough are the ones that are conducted long before it occurs.

Several empirical approaches were considered for predicting thermal breakthrough times from the simulated tracer tests at $0,10,000$, and $25,000 \mathrm{hr}$, all of which took place well before thermal breakthrough. The best approach found was based on the peak normalized tracer concentrations in each test and is described as follows:

1. Calculate the ratio of the peak concentration of the thermally degrading tracer to the peak concentration of the conservative tracer in each tracer test. Note that the test at time zero need not include a thermally degrading tracer because the reservoir temperature at this time is known to be the same everywhere (the initial reservoir temperature), so a thermally degrading tracer breakthrough curve can be calculated from a conservative tracer breakthrough curve if the Arrhenius decay parameters are known. The other tests should involve the simultaneous injection of both a conservative tracer and a thermally degrading tracer. The repeat injections of a conservative tracer are advisable because flow patterns may change over time as the reservoir cools.

2. Plot the times that each tracer test was conducted (since the start of cool water injection) vs. the logarithm (either natural or base 10) of the ratio of the peak tracer concentrations in each test, and then fit a second-order (quadratic) polynomial to the resulting data points. Note that the tests should be spaced as far apart as possible in time (within reason before thermal breakthrough) to maximize the accuracy of the extrapolation of the fit to a thermal breakthrough time.

3. Select or pre-calculate a ratio of peak tracer concentrations that is considered to correspond to thermal breakthrough. In the above example, a ratio of 1.0 would apply because the peaks were the same concentration at the time of thermal breakthrough.

4. Insert the logarithm of the thermal breakthrough ratio into the second-order polynomial regression equation from Step 2 to obtain a prediction of the thermal breakthrough time. 

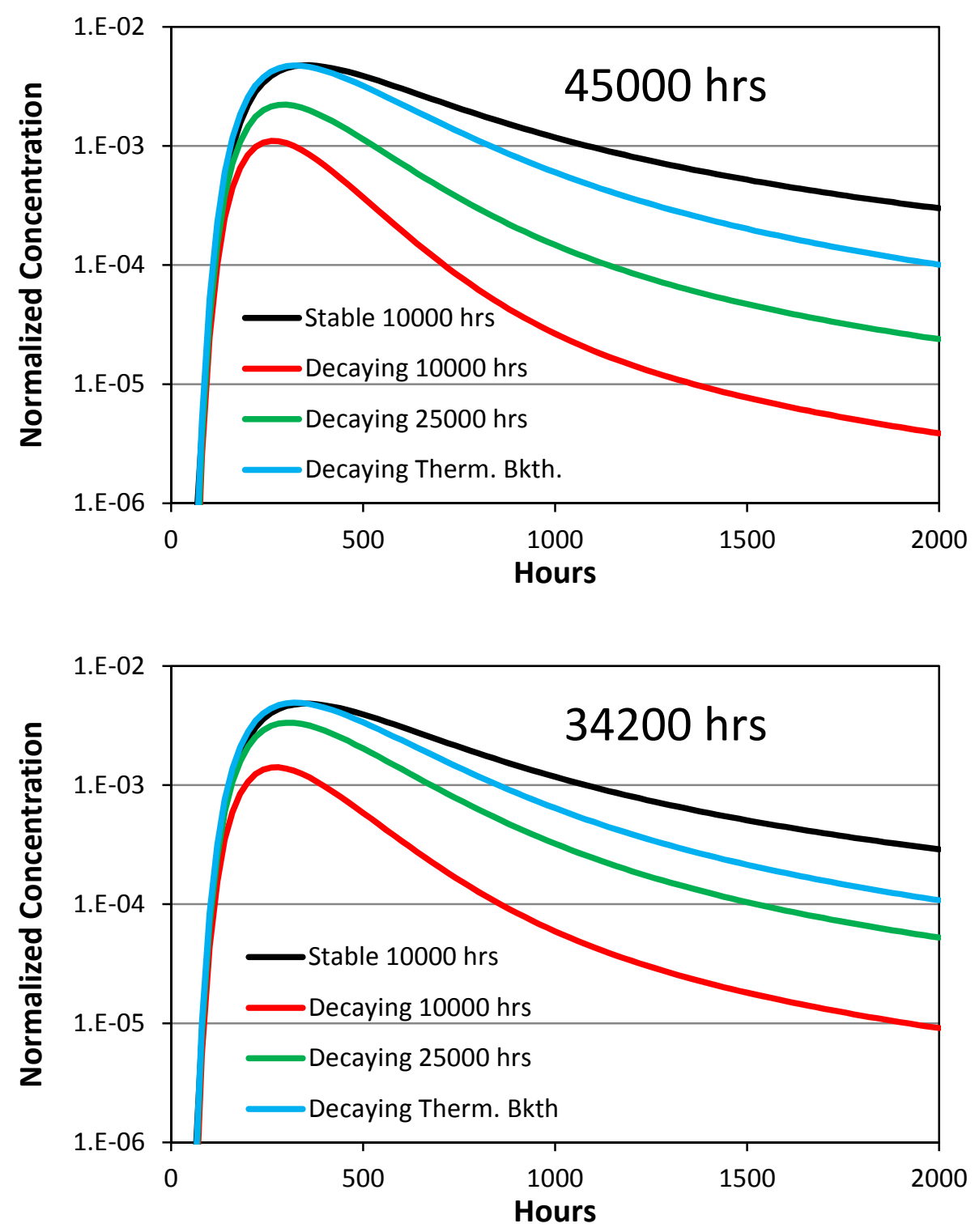

Figure 4.10. Tracer breakthrough curves for thermally stable and thermally decaying tracers in simulated tracer tests conducted at different times since the start of cold water injection for cases with a thermal breakthrough time of 45,000 hr (top) and 34,200 hr (bottom). Fracture spacing was assigned a standard deviation of $0.5 \mathrm{log}$ units, as in the cases of Figure 4.8.

An example of this approach is shown in Figure 4.11 for 1 of the 10 simulated geothermal systems. The thermal breakthrough time in this case is predicted to be the y-intercept of the second-order polynomial fit because $\ln (1)=0$. The resulting predicted and actual thermal breakthrough times for all 10 simulated geothermal systems are listed in Table 4.3. The agreement between the actual and predicted times is very good, with a standard deviation of differences of less than $5 \%$ of the actual thermal breakthrough time. The approach was also found to work well for the simulated geothermal systems of Figure 4.7 even though the thermal breakthrough in these cases occurred later because of the sharper thermal fronts (smaller thermal dispersion). Thus, the method appears to be quite robust regardless of 
thermal dispersion or how thermal breakthrough is defined. It is expected that other methods, such as a method based on ratios of areas under the tracer breakthrough curves or ratios of apparent mean residence times of tracers might also work quite well. However, peak concentrations are convenient in that they can be determined very precisely in the shortest amount of time. Also, if mean conservative tracer residence times or dispersivities change over time in a reservoir because of cooling-induced changes in fracture geometries, the ratio of the peak concentrations should remain relatively stable (similar to what they would have been without the cooling-induced changes). If mean residence times increase with time because cooling is widening fracture apertures (as expected), it can generally be assumed that the majority of the residence time increases are occurring in parts of the system where the greatest thermal drawdown has occurred and hence the least amount of thermal decay is occurring.

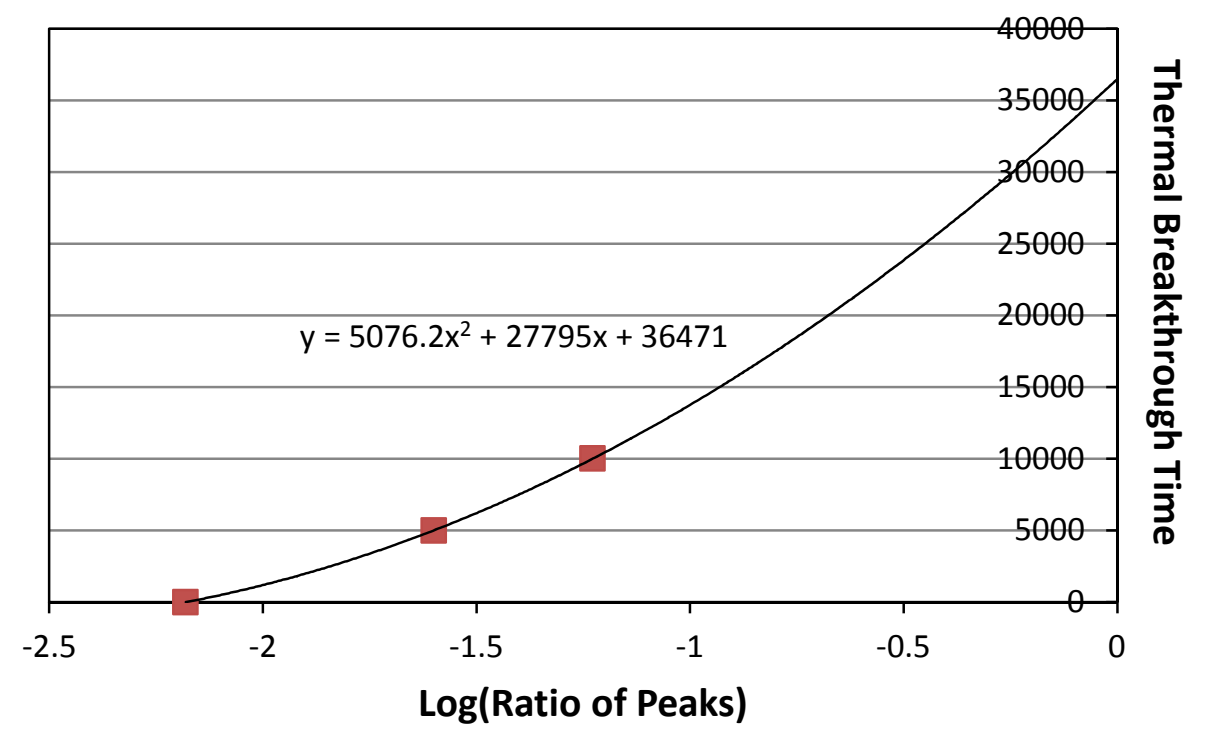

Figure 4.11. Example of the prediction of thermal breakthrough time from the y-intercept of a 2nd-order polynomial fit to the log of the ratio of a thermally decaying tracer peak concentration to non-decaying tracer peak concentration at different tracer test times.

Table 4.3. Actual (simulated) and predicted thermal breakthrough times using the method of Figure 4.11 (described in text).

\begin{tabular}{ccc}
\hline Actual (hr) & Predicted (hr) & Difference (hr) \\
\hline 44,400 & 44,239 & -161 \\
39,600 & 39,787 & 187 \\
43,200 & 46,000 & 2800 \\
36,000 & 33,789 & $-2,211$ \\
34,200 & 33,100 & $-1,100$ \\
44,400 & 46,878 & 2,478 \\
41,400 & 41,120 & -280 \\
45,000 & 47,035 & 2,035 \\
36,000 & 33,693 & $-2,307$ \\
33,600 & 33,515 & -85 \\
\hline
\end{tabular}


A requirement for this approach is that two tracer tests involving simultaneously injected conservative and thermally degrading tracers must be conducted (ratios from three tests are actually needed for the second-order polynomial fit, but the test at time zero can be conducted with just a conservative tracer because the thermally degrading tracer breakthrough curve can be calculated at time zero). It is also important that the decay characteristics of the thermally degrading tracer are such that the tracer does not decay completely away at the initial reservoir conditions over the mean tracer residence time in the system $(\ln (0)=-\infty$, which is impossible to fit). Likewise, the tracer should not have decay characteristics such that it exhibits no decay at all over the mean tracer residence time in the system before thermal breakthrough occurs. In this case, the thermal breakthrough time will always be over-predicted, which is an undesirable result. Finally, for systems with significant matrix porosity, and hence significant matrix diffusion, the conservative and thermally degrading tracers should ideally have similar diffusion coefficients so they experience approximately the same amount of matrix diffusion.

A good rule of thumb for thermally degrading tracer properties was found to be that 90 to $95 \%$ of the tracer should decay at the initial reservoir temperature over the mean tracer residence time in the system, and about 90 to $95 \%$ of the tracer should remain undecayed at a temperature equal to the injection temperature plus 50 to $65 \%$ of the difference between the injection temperature and the initial reservoir temperature. Fifty percent corresponds to a thermal breakthrough defined by a temperature drop at the production well of $\sim 5 \%$ of the original temperature difference, whereas $65 \%$ corresponds to a temperature drop at the production well of $\sim 0.5 \%$ of the original temperature difference. As tracer decay characteristics deviate from these rules of thumb, with either faster or slower than optimal tracer decay, predictions of thermal breakthrough times will become less reliable. Predictions will become more reliable as tracer tests are conducted closer to thermal breakthrough times because better information will be obtained when the reservoir has had more thermal drawdown. However, while waiting longer will provide a better estimate of the thermal breakthrough time, it has the disadvantage of providing less advance warning of thermal breakthrough and thus less time to plan for the thermal breakthrough. If one waits too long to conduct a final tracer test, thermal breakthrough may occur before the tracer test is started or completed. The difference in the thermally degrading tracer breakthrough curves in the first two tracer tests should provide some guidance about how long it would be prudent to wait for a final test. If there is very little difference between the breakthrough curves, then it is probably better to wait longer, but if there is significantly less thermal decay in the second test compared to the first, then a final test should be conducted sooner rather than later.

\subsection{Conservative Tracers with Different Diffusion Coefficients to Estimate Fracture Apertures or Surface Area-to-Volume Ratios}

The RELAP model was used to evaluate the potential to use simultaneously injected conservative (nonsorbing and non-thermally degrading) tracers with different diffusion coefficients to interrogate fracture apertures or SA/V ratios in interwell tracer tests. For parallel-plate fractures, SA/V $=1 / b$, where $b$ is the fracture half-aperture. The general idea with this approach is that conservative tracers with smaller diffusion coefficients (i.e., larger molecules/ions) will not as readily diffuse out of fractures and into the surrounding rock matrix as tracers with larger diffusion coefficients (smaller molecules/ions), so the breakthrough curves of the former tracers will tend to have higher peak concentrations and shorter tails than the latter tracers. The differences in the peak concentrations and tailing behavior of two such tracers is dependent on the ratio of the diffusion coefficients of the tracers, the matrix porosity, and the fracture $\mathrm{SA} / \mathrm{V}$ ratio or $1 / b$ in the flow system. Specifically, the differences are dictated by the lumped 
parameter, $\left(\phi_{m} / b\right) \cdot \sqrt{D_{m}}$, where $D_{m}$ is different for each tracer, but $\phi_{m}$ and $b$ are fixed and dependent only on the properties of the reservoir. Values for $\phi_{m}$ and $D_{m}$ can be estimated quite readily (although uncertainties can arise in estimating the porosity of rocks with extremely low porosity), and if these parameters are properly accounted for in an interpretive model, the differences between the breakthrough curves of the two tracers can be quantitatively translated into an estimate of the $\mathrm{SA} / \mathrm{V}$ ratio $(1 / b)$.

Figure 4.12 shows an example of the differences in simulated breakthrough curves as a function of fracture $\mathrm{SA} / \mathrm{V}$ ratio for three conservative tracers that have widely contrasting matrix diffusion coefficients. The model parameters other than the diffusion coefficients are the same as of Table 4.2. The three tracer diffusion coefficients roughly correspond to those of a halide (e.g., bromide or iodide), a naphthalene sulfonate, and a particle/colloid tracer with a free-water diffusion coefficient at $25^{\circ} \mathrm{C}$ of $2 \times 10^{-7} \mathrm{~cm}^{2} / \mathrm{s}$, which corresponds to a spherical particle about $20 \mathrm{~nm}$ in diameter (roughly equivalent to a large quantum dot tracer that is being developed by the University of Utah, Energy and Geosciences Institute). The diffusion coefficient contrast is approximately a factor of 4 for the halide and naphthalene sulfonate (free-water diffusion coefficients of $2 \times 10^{-5} \mathrm{~cm}^{2} / \mathrm{s} \mathrm{vs.} 5 \times 10^{-6} \mathrm{~cm}^{2} / \mathrm{s}$, respectively, at $25^{\circ} \mathrm{C}$ ) and approximately a factor of 25 for the naphthalene sulfonate and the particle tracer (or a factor of 100 for the halide and particle tracer). In all cases, the matrix diffusion coefficients at $25^{\circ} \mathrm{C}$ were assumed to be a factor of 20 smaller than the free-water diffusion coefficients. Figure 4.12 shows that there is a significant difference in peak concentrations of the tracers for a fracture half-aperture of $0.1 \mathrm{~cm}$, but this difference decreases for $b=0.25 \mathrm{~cm}$ and basically disappears for $b=1 \mathrm{~cm}$. However, there is still a difference in the tailing of the breakthrough curves for $b=1 \mathrm{~cm}$, so in principle the differences in the tracer breakthrough curves could still be used to interrogate fracture apertures.

Estimation of fracture apertures from breakthrough curves of tracers with different diffusion coefficients is not amenable to a simple empirical approach like that described in the previous section for estimating thermal breakthrough times. Rather, a model-fitting approach is needed. However, the approach is straightforward if the different tracers are injected simultaneously because under these conditions the model parameters will be the same for all tracers, and the differences in the breakthrough curves must be attributed to the differences in the diffusion coefficients. The lumped parameter $\left(\phi_{m} / b\right) \cdot \sqrt{D_{m}}$ is the actual matrix diffusion fitting parameter used to account for the differences in the breakthrough curves, so this parameter is adjusted simultaneously for each tracer, with the constraint that the known ratios of the matrix diffusion coefficients must be preserved. In the case of the three tracers of Figure 4.12, the ratio of diffusion coefficients is 100:25:1, which yields a ratio of 10:5:1 for the lumped parameter $\left(\phi_{m} / b\right) \cdot \sqrt{D_{m}}$. As Figure 4.12 shows, this ratio yields a relatively equal separation of peak concentrations, which is an ideal contrast to constrain the model-fitting exercise. Thus, the use of a halide, a naphthalene sulfonate, and a particle tracer with a diameter of 40 to $50 \mathrm{~nm}$ is an ideal set of three tracers to use for estimating fracture apertures based on matrix diffusion coefficient contrasts.

To obtain an actual estimate of $b$, the reservoir-average values of $\phi_{m}$ and $D_{m}$ must be known reasonably well ( $D_{m}$ for at least one of the three tracers) and inserted into the following expression:

$$
b=\frac{\phi_{m} \sqrt{D_{m}}}{\left[\left(\phi_{m} / b\right) \cdot \sqrt{D_{m}}\right]_{e x t}}
$$


Estimates of $b$ obtained this way will always have some uncertainty associated with them, but these uncertainties can be reduced by characterizing $\phi_{m}$ and $D_{m}$ and their variability within the reservoir as thoroughly as possible.
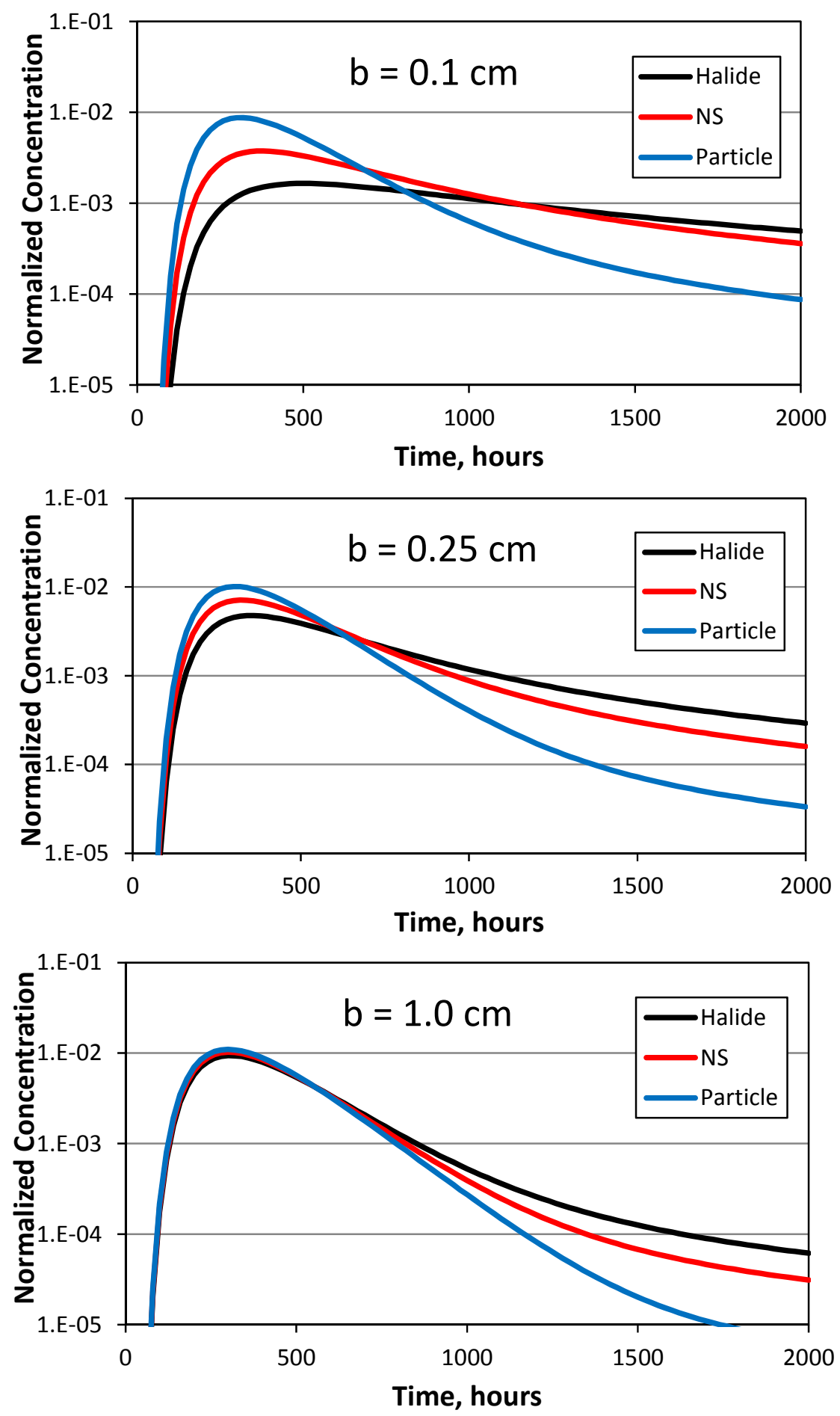

Figure 4.12. Simulated differences in breakthrough curves of conservative tracers with different matrix diffusion coefficients as a function of fracture half-aperture. Other model parameters are listed in Table 4.2. 
It should be noted that the method described above for estimating $b$ (or SA/V) assumes that 1 ) there is no fill material in the fractures, 2) the matrix has a constant matrix porosity in the direction perpendicular to the fractures, and 3) the fracture spacing is large enough that the tracers will diffuse only a small fraction of the distance between the fractures. These assumptions can be relaxed in models and still allow the method to be applied rigorously, but the additional uncertainties associated with these realsystem complexities impart additional uncertainty to the estimation of $b$. Fracture mineral coatings could especially impart considerable uncertainty to estimates of $b$, because they could result in relatively thin and/or uneven layers on fracture surfaces of material of different porosity than the bulk matrix, which would significantly affect matrix diffusion. Diffusion might also occur into stagnant free water in fractures and be interpreted as matrix diffusion, which will cause an underestimation of $b$ or an overestimation of SA/V. For rough-walled fractures and channelized flow in fractures there will always be some tracer diffusion into stagnant free water. The assumption of parallel-plate fractures is made for convenience because the actual spatial variability of fracture apertures and the degree of flow channelization can never be known with precision, but this assumption clearly results in errors and biases in aperture estimates when using the diffusion coefficient contrast method. The method also has limitations in rocks of extremely low matrix porosity, as for any given tracer mean residence time in fractures, there will be some matrix porosity below which insufficient separation will be observed in the tracer breakthrough curves to obtain an estimate of $b$. Nevertheless, despite these caveats and limitations, the diffusion coefficient method is useful as one of the few tracer methods available for estimating fracture apertures.

\subsection{Adsorbing Tracers to Estimate Fracture Apertures or Surface Area-to-Volume Ratios}

The RELAP model was used to evaluate the potential to use simultaneously injected conservative and adsorbing tracers to interrogate fracture apertures or SA/V ratios in interwell tracer tests. This approach involves an additional level of complexity and/or uncertainty over that of the diffusion coefficient method of the previous section because adsorbing tracers also diffuse, so both adsorption and diffusion must be accounted for when estimating $b$. Furthermore, adsorption is inherently more complex than diffusion, often involving nonlinear processes, and it is likely to have more spatial variability than diffusion. However, an advantage of adsorption as a process affecting tracer transport is that it can result in much greater contrast with a conservative tracer than diffusion provides, thus in principle offering a more sensitive measure of fracture apertures or SA/V ratios based on differences in tracer breakthrough curves. Also, adsorbing tracers can provide estimates of fracture apertures even in situations where diffusing tracers fail; for instance, when there is negligible matrix porosity in a reservoir.

To avoid undue complexity, we limit the following discussion to the use of adsorbing tracers that adsorb rapidly, linearly, and reversibly; that is, tracers for which equilibrium partitioning between the solution and solid surfaces can be assumed to occur everywhere in the system and the partitioning is such that the ratio of adsorbed tracer mass to tracer mass in solution varies only with the solid surface area to solution volume ratio, not the tracer concentration in solution. In truth, adsorbing tracers with these characteristics are highly desirable to simplify what is already a complex inverse problem of estimating fracture apertures from adsorbing tracer responses. The use of tracers that exhibit rate-limited adsorption, nonlinear adsorption isotherms (for which ratios of adsorbed mass to mass in solution are dependent on tracer concentrations in solution), and partially irreversible adsorption should be avoided to the extent possible because of the non-uniqueness in tracer test interpretations that these characteristics can cause. 
One possible exception is the use of cation-exchanging tracers, which have notoriously nonlinear adsorption isotherms. However, these nonlinearities, in conjunction with their very rapid and reversible adsorption behavior, make them potentially quite useful for estimating fracture apertures in single-well injection/withdrawal tracer tests, as discussed in Chapter 5.0.

When fast, linear, and reversible tracer adsorption occurs in the matrix in a dual-porosity system, it has the simple and useful effect of enhancing the effective matrix diffusion coefficient of the tracer by a factor equal to $R_{m}$ (see Equation (4.4)). In this case, the lumped parameter for matrix diffusion introduced in the previous section becomes $\left(\phi_{m} / b\right) \cdot \sqrt{R_{m} D_{m}}$, from which it is obvious that $R_{m}$ is a multiplier that effectively increases the matrix diffusion coefficient. RELAP model simulations confirmed that a matrix retardation factor of 2 had the same effect on simulated tracer transport as doubling the tracer matrix diffusion coefficient and assuming a retardation factor of 1.0. However, this linear enhancement is only valid if the matrix is uniform in porosity and mineralogy. Nonetheless, this enhancement can be very useful, particularly for low-porosity matrices, because it makes it possible to use tracers with much larger effective diffusion coefficients than nonsorbing tracers can physically provide (these have an upper limit for the free-water diffusion coefficient of about 2 or $3 \times 10^{-5} \mathrm{~cm}^{2} / \mathrm{s}$ at $25^{\circ} \mathrm{C}$ ).

Adsorbing tracers are also likely to adsorb to fracture surfaces or fill material present in fractures, which will impart a classic delay to their transport in fractures in addition to any delays caused by diffusion and adsorption in the matrix. This fracture delay process is mathematically represented by the fracture retardation factor $R_{f}$ in Equation (4.3), where $R_{f}$ is given by

$$
R_{f}=1+\frac{K_{a}}{b}+\frac{\rho_{R \eta}(1-\eta) K_{d}}{\eta}
$$

where the definitions are provided after Equations (4.3) and (4.4).

For an unfilled parallel-plate fracture with $\eta=1$, Equation (4.20) reduces to $R_{f}=1+\frac{K_{a}}{b}$, which implies that if the surface-area-based partition coefficient $K_{a}$ is known, and the retardation factor is obtained from a tracer test, the fracture half-aperture, $b$, can be directly estimated. In practice, diffusion and adsorption in a thin porous layer lining the surface of a fracture can look exactly like adsorption onto fracture walls, especially when the layer is thin enough that tracers can readily diffuse across it during the mean tracer residence time in the system. In this case, the effective fracture retardation factor becomes

$$
R_{f}=1+\frac{\phi_{l} h_{l} R_{l}}{b}
$$

where $h_{l}$ is the thickness of porous fracture lining, cm; $R_{l}$ is $1+\frac{\rho_{R_{l}}\left(1-\phi_{l}\right) K_{d l}}{\phi_{l}}$; and $l$ is the subscript denoting lining property. 
Figure 4.13 and Figure 4.14 show how even a small effective retardation factor in the fractures, $R_{f}$, can greatly help in interrogating fracture apertures. In Figure 4.13, a $K_{a}$ or $\phi_{l} h_{l} R_{l}$ value of $0.125 \mathrm{~cm}$ was used with no fracture fill material assumed, and a matrix diffusion coefficient of $1 \times 10^{-6} \mathrm{~cm}^{2} / \mathrm{s}$ at $25^{\circ} \mathrm{C}$ was assumed for the conservative and adsorbing tracers. It was assumed that no adsorption occurred in the matrix (i.e., adsorption only occurred in a thin porous fracture lining), although for this relatively small amount of adsorption, retardation in the matrix equivalent to that in the fracture lining would have increased the effective matrix diffusion coefficient by only a factor of 3 or so. In Figure 4.14, all parameters were the same as in Figure 4.13 except that a matrix diffusion coefficient of $1 \times 10^{-8} \mathrm{~cm}^{2} / \mathrm{s}$ at $25^{\circ} \mathrm{C}$ was assumed for the conservative and adsorbing tracers, which nearly eliminates the effects of matrix diffusion while preserving the effect of adsorption on or along the fracture walls. All other model parameters in both cases were the same as those listed in Table 4.2.

It is apparent from Figure 4.13 and Figure 4.14, that the delay in the arrival of the adsorbing tracer relative to the conservative tracer is inversely proportional to the fracture aperture regardless of the amount of matrix diffusion (or by extension, regardless of the amount of combined diffusion and adsorption in the matrix). Even the relatively small amount of adsorption to fracture walls or linings depicted in these figures could be exploited to estimate fracture apertures. However, quantitative estimates of apertures will require a thorough characterization of fracture properties and adsorption processes within fractures. For instance, in a blind tracer test one could attribute the same adsorbing tracer breakthrough curve to either a small amount of adsorption in a small aperture fracture or a large amount of adsorption in a large aperture fracture. Tracer retardation factors tend to be more variable and uncertain than tracer matrix diffusion coefficients because chemical heterogeneity in subsurface systems is typically greater than physical heterogeneity, and both types of heterogeneity affect tracer retardation factors while only physical heterogeneity affects diffusion coefficients. The use of more than one adsorbing tracer, with each having different retardation factors, is expected to be of limited benefit because if adsorption in the reservoir is higher or lower than expected for one adsorbing tracer (thus biasing fracture aperture estimates), it is likely to be higher or lower than expected for all adsorbing tracers. However, using two different adsorbing tracers with different amounts of adsorption would extend the dynamic range of fracture apertures that could be interrogated by increasing the likelihood that at least one of the tracers will provide good breakthrough curve separation from a conservative tracer. For instance, the amount of adsorption to fracture walls or linings in Figure 4.13 and Figure 4.14 is almost too small to interrogate fracture half-apertures of $1 \mathrm{~cm}$ or greater, so a tracer that adsorbs more strongly would be desirable for interrogating larger apertures. Another beneficial use of multiple adsorbing tracers would be if multiple tracers could be found that adsorb differently in the fractures and the matrix (due to mineralogical differences in the fractures and matrix), because this would allow some distinction between the effects of adsorption in the two domains, thus allowing for more quantitative tracer test interpretations.

Adsorption to fracture walls or linings offers advantages over adsorption strictly to matrix surfaces because it imparts a more notable delay in both the first arrival time and peak arrival time of the adsorbing tracer relative to a conservative tracer. This delay is much more easily and precisely translated into a fracture half-aperture than a mere lowering of concentrations and increased tailing caused by adsorption in the matrix. In most situations, adsorption will occur both in fractures and the matrix, but because the time delay is more pronounced from adsorption in fractures, it is generally possible to achieve some degree of separation between adsorption in the two domains when interpreting tracer breakthrough curves (e.g., Reimus et al. 2003). 

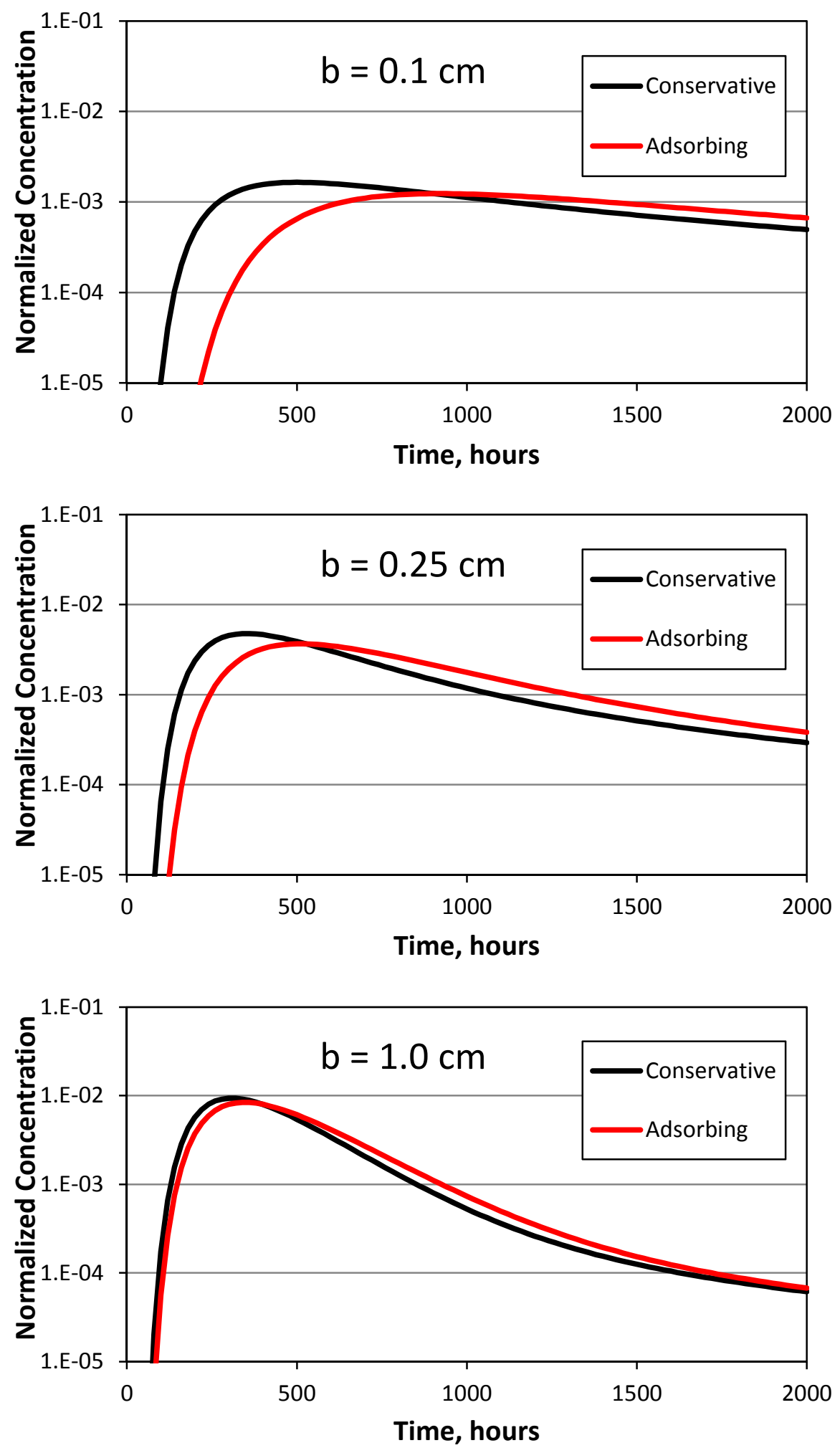

Figure 4.13. Simulated differences in breakthrough curves as a function of fracture half-aperture for conservative and adsorbing tracers having matrix diffusion coefficients of $1 \times 10^{-6} \mathrm{~cm}^{2} / \mathrm{s}$ at $25^{\circ} \mathrm{C}$. $K_{a}$ or $\phi_{l} h_{l} R_{l}$ value for adsorbing tracer assumed to be $0.125 \mathrm{~cm}$. Other model parameters are listed in Table 4.2. 

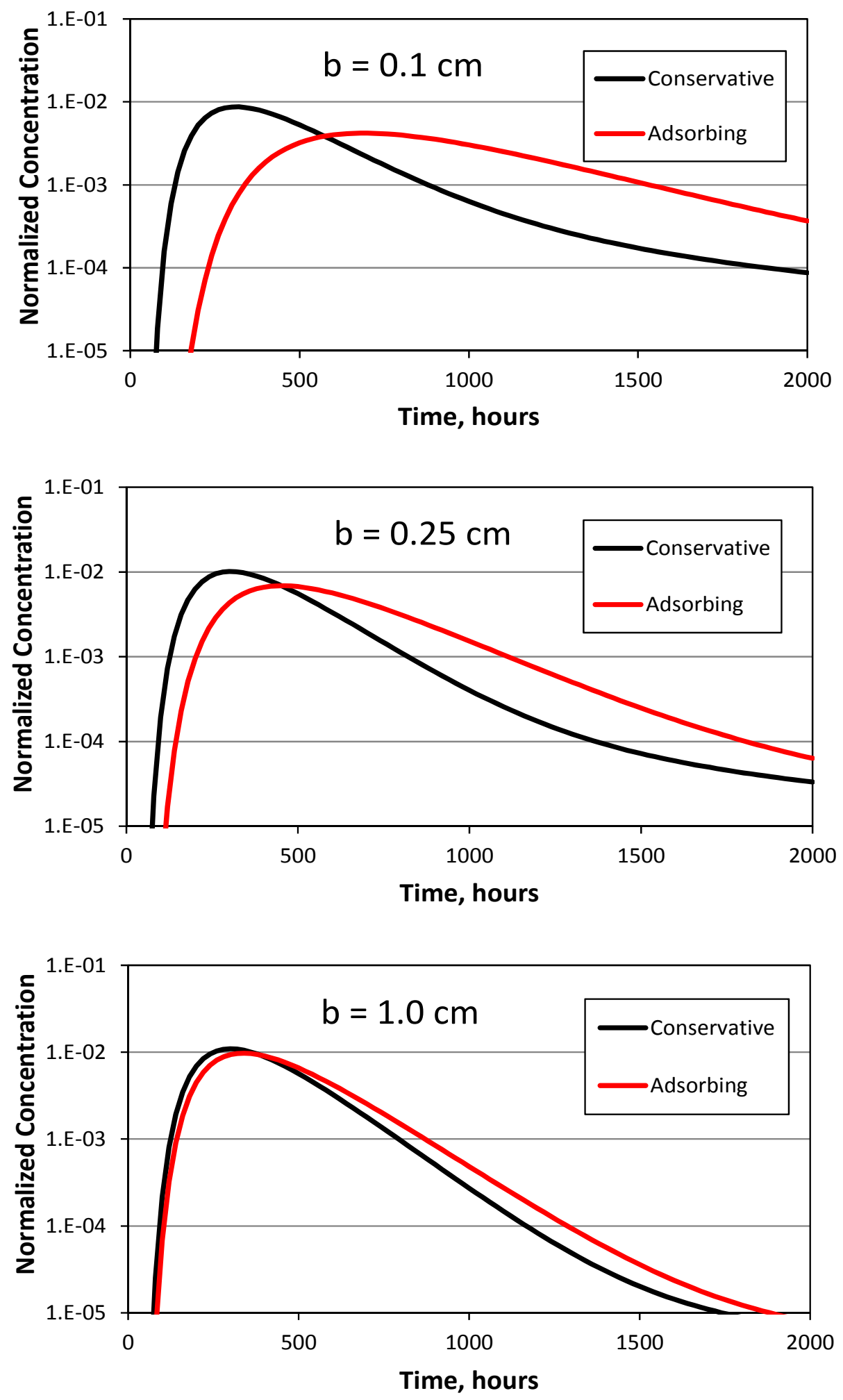

Figure 4.14. Simulated differences in breakthrough curves as a function of fracture half-aperture for conservative and adsorbing tracers having matrix diffusion coefficients of $1 \times 10^{-8} \mathrm{~cm}^{2} / \mathrm{s}$ at $25^{\circ} \mathrm{C}$. $K_{a}$ or $\phi_{l} h_{l} R_{l}$ value for adsorbing tracer assumed to be $0.125 \mathrm{~cm}$. Other model parameters are listed in Table 4.2. 
Probably the biggest uncertainty associated with translating the observed fracture component of adsorption into a fracture half-aperture estimate is the uncertainty in amount of adsorption that is occurring to fracture fill material (the last term on the right-hand side in Equation (4.20)) as opposed to fracture walls or linings. As Equation (4.20) suggests, retardation as a result of adsorption to fracture fill material is independent of fracture half-apertures, so a significant amount of adsorption to fill material will make the observed fracture retardation of an adsorbing tracer less sensitive to fracture half-apertures

\subsection{Combined Use of Tracers in Interwell Tracer Tests to Estimate Flowing Fracture Spacings}

As discussed in the previous sections, the use of diffusing and/or adsorbing tracers in interwell tracer tests offers the potential to interrogate fracture apertures or SA/V ratios in geothermal reservoirs provided some physical and chemical characterization of the fractures and rock matrix is available. In Section 4.2, it was shown that thermally degrading tracers can be used to estimate when thermal breakthrough will occur in a geothermal reservoir. Given that thermal breakthrough times are proportional to the quantity(ies) on the right-hand side of Equations (4.17) and (4.18), which contain both the average fracture half-aperture $b$ and the average fracture spacing $L$, it follows that estimates of fracture halfapertures via the use of diffusing and/or adsorbing tracers and estimates of thermal breakthrough times via the use of thermally degrading tracers can be used together to obtain estimates of average flowing fracture spacing in a reservoir. The rock and water thermal properties in Equations (4.17) and (4.18) are generally quite well known and will typically not vary nearly as much as mass transport properties in the fractures and matrix, so the uncertainty in estimated fracture spacing associated with this approach will be dominated by uncertainties in fracture half-aperture estimates (discussed in the preceding section). No simulations were conducted to test this approach, but the principles behind it are sound. However, the practical value of this method for estimating fracture spacing is questionable because it requires an estimate of thermal breakthrough time before a fracture spacing estimate can be obtained, and the primary value of estimating fracture spacing is to allow a better prediction of thermal breakthrough time. Thus, obtaining a good estimate of thermal breakthrough time prior to estimating fracture spacing effectively defeats the purpose of obtaining fracture spacing estimates. 


\subsection{Multicomponent Numerical Model for Heat and Mass Transport in Interwell and Single-Well Tracer Tests, Including Cation-Exchange Reactions}

The MULTRAN computer model is described first below, followed by descriptions of temperature as a tracer in single-well tests to estimate flowing fracture spacings, the use of diffusing and adsorbing tracers to estimate fracture apertures or SA/V ratios in single-well tracer tests, and the special case of cation-exchanging tracers in single-well tracer tests.

\subsection{Model Description}

The MULTRAN computer model (Sullivan et al. 2003) was used to conduct exploratory simulations to evaluate the potential for using cation-exchanging tracers to interrogate surface area in both interwell and single-well tracer tests in EGS reservoirs. The modeled system is assumed to be a dual-porosity medium with parallel-plate fractures and rectangular matrix blocks (i.e., the geometry of Figure 4.1). For this work, MULTRAN was also upgraded to include the capability to simulate heat transport in EGS reservoirs (Equations (4.12) and (4.13)) and to account for the temperature dependence of diffusion coefficients (Equation (4.16)) as well as the temperature dependence of reaction rates or reaction equilibrium constants. Spatial variability in properties such as matrix porosity, matrix diffusion coefficients, and CECs are also supported. The numerical model allows the restrictions imposed by the Laplace transform solution of RELAP (most notably no interacting species and steady-state flow conditions) to be relaxed, thus allowing MULTRAN to simulate the flow interruptions and flow reversals associated with single-well tracer tests, as well as providing additional flexibility in simulating interwell tracer tests that have non-steady flow conditions.

MULTRAN simulates the transport of cation-exchanging tracers by solving mass-action equations for cation-exchange equilibria simultaneously with the transport equations. The cation-exchange reactions are given by (using the Gaines-Thomas convention) the following:

$$
N a^{+}+\frac{1}{z_{C}} C X_{Z_{C}} \rightarrow N a X+\frac{1}{z_{C}} C^{z_{C}^{+}}
$$

where $C$ is a cation other than $\mathrm{Na}, z_{C}$ is a positive integer charge of cation $C$, and $X$ is a surface site with charge of -1 .

The equilibrium expressions, or mass-action equations, for reactions (5.1) are given by the following:

$$
K_{N a / C}=\frac{[N a X]\left[C^{z_{C}^{+}}\right]^{1 / z_{C}}}{\left[N a^{+}\right]\left[C X_{z_{C}}\right]^{1 / z_{C}}}
$$

where $K_{\mathrm{Na} / \mathrm{C}}$ is called the selectivity coefficient, which effectively provides a measure of the ability of $\mathrm{Na}$ to displace cation $C$ from a cation-exchange site on the surface. In general, a smaller value of $K_{\mathrm{Na} / \mathrm{C}}$ will result in cation $C$ being more likely to displace other cations from the surface. 
The mass-action equation (Equation (5.2)) for each cation $C$ are solved simultaneously along with the overall surface charge balance equation (Equation (5.3)), to describe cation-exchange equilibria in the system.

$$
C E C=\frac{\phi}{\rho_{B}} \sum_{\text {all } C}\left[C X_{z_{C}}\right]
$$

where $\mathrm{CEC}=$ cation-exchange capacity, eq/kg.

Flow in the fracture domain is allowed to be specified as either linear or radial, with the latter being used to simulate single-well tracer tests. For radial flow, Equation (4.1) is replaced with the following equation:

$$
\frac{\partial C_{i}}{\partial t}+V(r) \frac{\partial C_{i}}{\partial x}-\frac{1}{r} \frac{\partial}{\partial r}\left(r D \frac{\partial C_{i}}{\partial r}\right)-\left.\frac{\phi_{m}}{\eta b} D_{m} \frac{\partial C_{i, m}}{\partial y}\right|_{y=b}=0
$$

where, $C_{i}$ refers to the concentration of any cation or anion (not necessarily just a cation as in the above equations).

Multicomponent diffusion in the y-direction in the matrix is more complex than single-component diffusion, so Equation (4.2) is replaced with the following set of equations for all species except the $n$th species, where $n$ is the total number of species (Newman 1973):

$$
\frac{\partial c_{i}}{\partial t}=D_{i} \nabla^{2} c_{i}-\sum_{j} \frac{z_{j}}{z_{i}}\left(D_{j}-D_{n}\right) \nabla \cdot\left(t_{i} \nabla c_{i}\right)
$$

where $\quad c_{\mathrm{i}}=$ molar concentration of species $i$, moles $/ \mathrm{L}$

$$
\begin{aligned}
D_{i} & =\text { diffusion coefficient of species } i, \mathrm{~cm}^{2} / \mathrm{s} \\
\nabla & =\text { del operator } \\
\nabla^{2} & =\text { Laplacian operator } \\
t_{\mathrm{i}} & =\frac{z_{i}^{2} u_{i} c_{i}}{\sum_{j} z_{j}^{2} u_{j} c_{j}}=\text { transference number of species } i \\
z_{i} & =\text { charge of species } i \\
u_{i} & =\frac{D_{i}}{R T}=\text { mobility of species } i, \text { where } R=\text { gas constant and } T=\operatorname{temp}(\mathrm{K}) .
\end{aligned}
$$

The concentration of the $n$th species is then given by the electroneutrality or charge balance constraint in solution:

$$
z_{n} c_{n}=-\sum_{j \neq n} z_{j} c_{j}
$$


MULTRAN solves Equations (5.1) through (5.6) simultaneously along with Equations (4.12) and (4.13) to provide a mass and heat transport solution for a multicomponent cation-exchanging system in a dual-porosity medium having the geometry of Figure 4.1.

In the remainder of this chapter, we use the MULTRAN code to explore the potential to use diffusing and adsorbing (especially cation-exchanging) tracers to interrogate SA/V ratios in single-well tests in geothermal reservoirs. We also explore the potential use of temperature as a tracer to interrogate reservoir properties in single-well tests. The use of cation-exchanging tracers for surface area interrogation is appealing because these tracers do not thermally degrade at geothermal reservoir temperatures like many fluorescent dye tracers do, but they have disadvantages in that they tend to have higher detection limits than fluorescent tracers, and they also have significant background concentrations in many geothermal reservoirs. These disadvantages make cation-exchanging tracers impractical for interwell tracer tests (unless radioactive isotopes are used) because large dilution factors are typically observed in such tests. However, these disadvantages are much less of an issue in single-well tracer tests because tracer concentrations tend to remain much higher in these tests.

\subsection{Temperature as a Tracer in Single-Well Tests to Estimate Flowing Fracture Spacings}

Pruess and Doughty (2010) explored the possibility of using temperature as a tracer in single-well injection-withdrawal tests, and they concluded that temperature did not provide a very good indicator of fracture apertures, but it had the potential to provide a good indicator of changes in surface area independent of changes in volume within the system. Thus, if the SA/V ratio increases as a result of a decrease in fracture aperture (decrease in volume) without any change in surface area, then the temperature history during the withdrawal phase of the single-well test will not change. However, if the $\mathrm{SA} / \mathrm{V}$ ratio increases as a result of an increase in surface area while the volume stays the same (corresponding to an offsetting decrease in fracture aperture to maintain the same volume), then the temperature history will change, with higher temperatures seen initially during withdrawal.

Rather than explore the ability to interrogate surface area, we chose to evaluate the potential to use temperature as a tracer to interrogate flowing fracture spacings in single-well injection/withdrawal tests. Simulations were conducted using MULTRAN with the fracture aperture, spacing, and volumetric flow rates in each fracture, and the total time of injection prior to withdrawal varied. Figure 5.1 shows the temperature histories for 5 tests with 50 -hr injections of $25^{\circ} \mathrm{C}$ water into a $250^{\circ} \mathrm{C}$ reservoir, followed by 2 -hr rest periods before withdrawal. In these simulations volumetric flow rates, fracture apertures, and fracture spacings were varied. Note that although the higher flow rates and smaller apertures tended to result in slightly cooler temperatures near the wellbore during the injection phase, the return temperatures are all essentially identical. This result was somewhat surprising because the tests at different flow rates with the same aperture or at the same flow rate with different apertures should have had different overall surface areas in contact with the injected water. The difference between these simulations and those of Pruess and Doughty (2010) is that the extra surface area in the case of the simulations here is farther into the reservoir from the wellbore rather than being more surface area available over the same distance from the wellbore. The temperature curves of Figure 5.1 all have the same shape during the return flow, but the actual heat extracted is greater in the case of the higher volumetric flow rates because more water is extracted over the same time period. 


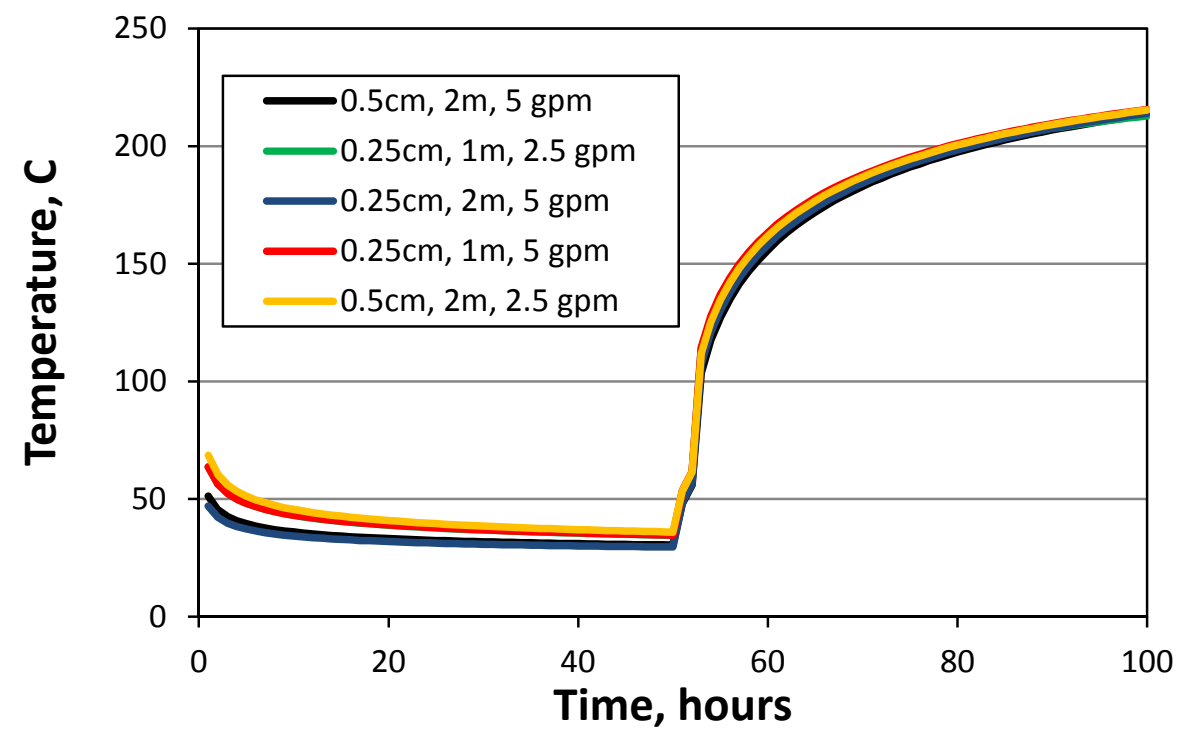

Figure 5.1. Wellbore temperature histories in single-well tests with 50 -hr injection of $25^{\circ} \mathrm{C}$ water into a $250^{\circ} \mathrm{C}$ reservoir followed by $2-\mathrm{hr}$ rest period as a function of fracture half-aperture (first number in legend), fracture half-spacing (second number), and volumetric flow rate in and out (third number). System thermal properties are approximately that for a basalt.

Figure 5.2 shows temperature histories for the same set of flow rates, apertures, and fracture spacings but for 250-hr injections followed by 2 -hr rest periods before withdrawal, and also with one additional case included that has a fracture spacing of $6 \mathrm{~m}$. In this figure, there is a difference between the temperature curves for different fracture spacings, but not for any of the other variables. The curves from Figure 5.1 are also shown for comparison. It is of interest that the return temperature curves for the 4-m and 6-m fracture spacings are nearly identical, but the curves for the 2-m fracture spacing are significantly lower. These results suggest that the rock near the wellbore that is in contact with the water that is first produced has cooled to the point that it is no longer keeping the early-produced water as warm as in the cases with larger fracture spacings. The cooling rate of the rock and heating rate of the water is dictated primarily by heat conduction out of the rock and this conduction acts to cool the rock a distance into the matrix that is proportional to the square root of time, with the dependence being approximately

$$
X=\sqrt{2 D_{T} t}
$$

where $X$ is the distance of cooling front into the rock; $D_{T}$ is the thermal diffusivity $=k / \rho C_{p}(k=$ thermal conductivity, $\rho=$ density, $C_{p}=$ heat capacity), which for the cases of Figure 5.1 and Figure 5.2 was assumed to be $0.01 \mathrm{~cm}^{2} / \mathrm{s}$; and $t$ is the time.

For times of $50 \mathrm{hr}$ and $250 \mathrm{hr}, X$ in Equation (5.7) is equal to $60 \mathrm{~cm}$ and $134 \mathrm{~cm}$, respectively. Thus, when the fracture spacing is $2 \mathrm{~m}$, the rock near the wellbore can be expected to be cooled to the middle of the matrix blocks after $250 \mathrm{hr}$, but it is not cooled to the middle of the blocks after only $50 \mathrm{hr}$. Also, in the case of the 4-m or 6-m fracture spacings, the rock is not cooled to the middle of the blocks even after $250 \mathrm{hr}$, which explains why there is only a minor difference between the temperature return curves for these two spacings in Figure 5.2. Taken together, the temperature return curves at $50 \mathrm{hr}$ and $250 \mathrm{hr}$ in 
Figure 5.2 indicate that the distance to the center of the matrix blocks in all cases must exceed the thermal diffusion distance after $50 \mathrm{hr}(60 \mathrm{~cm})$, but the distance to the center of the blocks for the smallest fracture spacing is less than the thermal diffusion distance after $250 \mathrm{hr}(134 \mathrm{~cm})$.

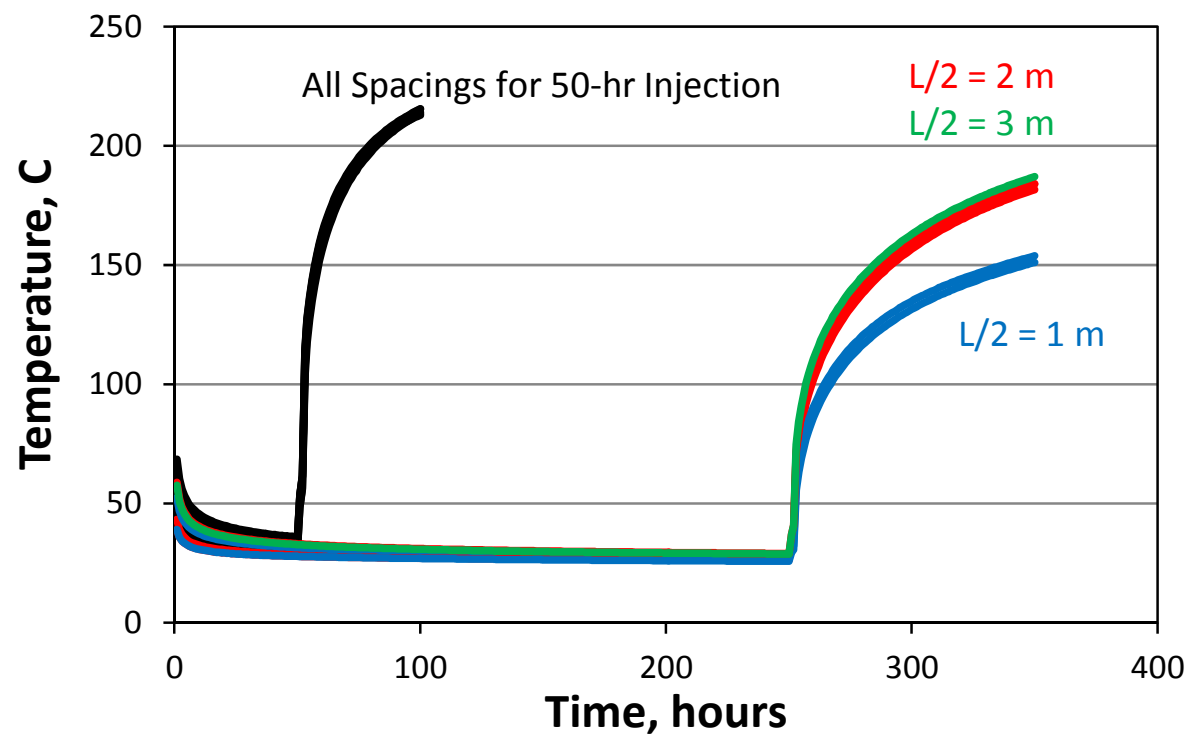

Figure 5.2. Wellbore temperature histories in single-well tests with 50-hr injection and 250-hr injection of $25^{\circ} \mathrm{C}$ water into a $250^{\circ} \mathrm{C}$ reservoir followed by $2-\mathrm{hr}$ rest period in each case. Curves are shown for different half-apertures, fracture half-spacings, and volumetric flow rates, but only the half-spacings have an effect, and then only for the cases with $250-\mathrm{hr}$ injections. System thermal properties are approximately that for a basalt.

These results suggest that temperature return curves after known injection times in single-well injection/withdrawal tests have the potential to provide bounding estimates for effective flowing fracture spacings near wellbores. The method relies on inverse modeling and would work best if two injection/withdrawal tests were conducted with significantly different injection time periods. Ideally, the first test would be a relatively short injection with a short rest period so that the rock near the wellbore is not cooled significantly before conducting a second test. This test would be expected to have the same temperature return curve regardless of fracture spacing (unless the spacing is extremely small), and its result would be used to predict what the temperature return curves should look like for different assumed fracture spacings with a much longer injection. Note that for a given injection time all return curves will be predicted to be the same when the spacing exceeds some value that is comparable to the value of $X$ given by Equation (5.7). The longer injection test would then be conducted, and the test results could be compared to the model predictions (optionally accounting for the cooling in the first test) to determine whether they are consistent with any of the modeled fracture spacings. It may be possible only to say that the effective flowing fracture spacing exceeds some lower bound that the method cannot interrogate beyond for the given injection time period, but this may still be valuable information to the practitioner. If the return curves indicate a smaller fracture spacing than the lower bound of interrogation, the implication would be that the reservoir may exhibit faster-than-desirable thermal drawdown because of relatively small effective fracture spacings. 
It must be kept in mind that this method will only interrogate a relatively short distance into the reservoir from the injection well, and thus it may not provide fracture spacing estimates that are representative of the entire reservoir between an injection and production well. Even so, the estimates should represent much larger volumes than can be interrogated using typical wellbore geophysical methods. The results presented here should be considered preliminary, because there was no attempt to evaluate the robustness or uncertainties associated with the method.

\subsection{Diffusing and Adsorbing Tracers to Estimate Fracture Apertures or Surface Area-to-Volume Ratios in Single-Well Tracer Tests}

As in the case of interwell tracer tests, conservative tracers with different diffusion coefficients can be used to interrogate fracture half-apertures or SA/V ratios in single-well tracer tests. More diffusive tracers will have lower peak concentrations and longer tails than less diffusive tracers, with the magnitude of the differences being greater for smaller average fracture half-apertures (assuming fixed values of matrix porosity and tracer matrix diffusion coefficients). Figure 5.3 shows the simulated single-well tracer return curves in a $200^{\circ} \mathrm{C}$ reservoir of three conservative tracers with different diffusion coefficients for three different assumed fracture half-apertures. The three tracers were assigned the same values of matrix diffusion coefficients as those simulated in Section 4.3 for interwell tracer tests; i.e., corresponding to a halide, a naphthalene sulfonate, and a $40-\mathrm{nm}$-diameter particle tracer. The matrix porosity was also assumed to be the same (0.05) as in the simulations of Section 4.3. In this case, the tracer injection was simulated for $5 \mathrm{hr}$, followed by injection of tracer-free water for $45 \mathrm{hr}$, followed by a 2-hr rest period, and then extraction/withdrawal at the same rate as injection.

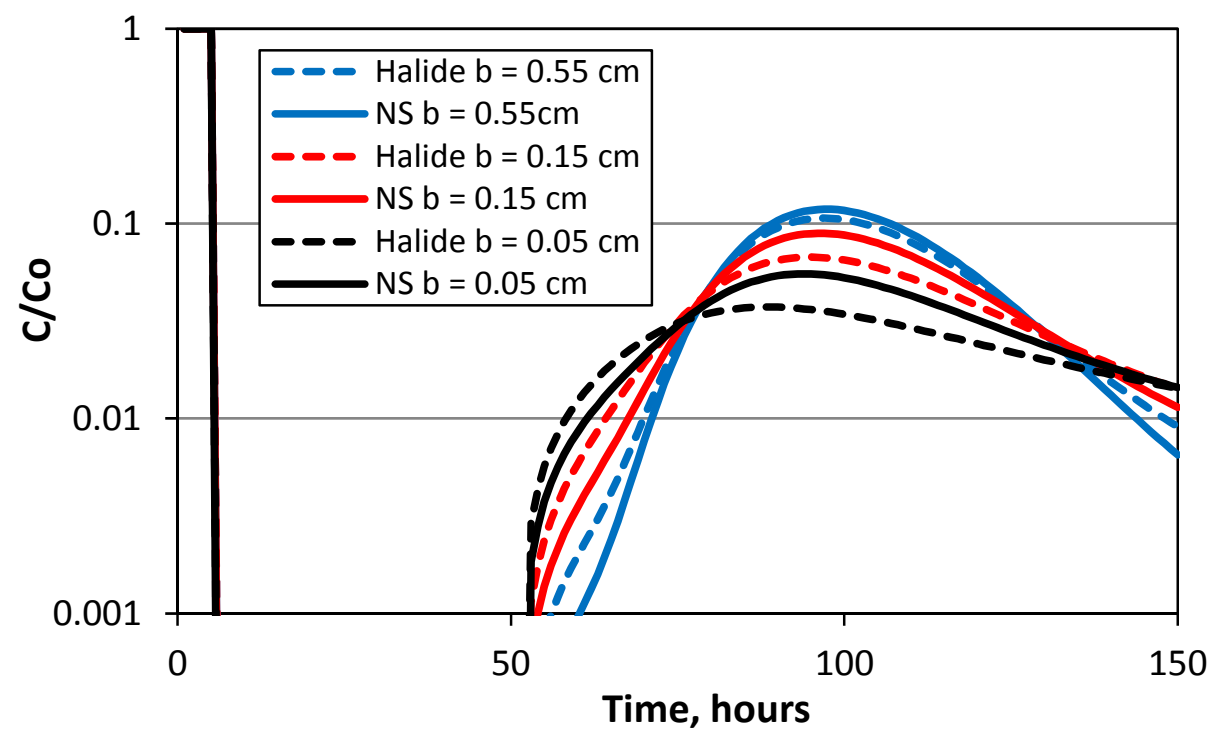

Figure 5.3. Simulated single-well breakthrough curves of conservative tracers with different diffusion coefficients as a function of fracture half-aperture in a $200^{\circ} \mathrm{C}$ reservoir. Tracers injected for $5 \mathrm{hr}$, followed by injection of tracer-free water for $45 \mathrm{hr}$, followed by a 2-hr rest period before withdrawal. Matrix porosity $=0.05$. 
The differences between the tracer return curves in Figure 5.3 are quite striking, especially considering the log concentration scale for the y-axes. It is apparent that these differences should provide well-constrained estimates of fracture half-apertures if the matrix porosity and tracer matrix diffusion coefficients are known reasonably well. Of course, as with interwell tracer tests, this method of estimating fracture apertures will break down if the matrix porosity is very low and there is consequently very little difference between the breakthrough curves of the different tracers.

In the case of adsorbing tracers, fast, linear, and reversible adsorption in the matrix has the same effect of increasing the effective matrix diffusion coefficients of the tracers as in interwell tracer tests (Section 4.4). Figure 5.4 shows the simulated response of an adsorbing tracer with a partition coefficient of $0.5 \mathrm{ml} / \mathrm{g}$ relative to a conservative tracer with the same diffusion coefficients as a function of fracture half-aperture in single-well tracer tests in a $200^{\circ} \mathrm{C}$ system with a matrix porosity of 0.05 . The simulation parameters are otherwise the same as in the simulations of Figure 5.3.

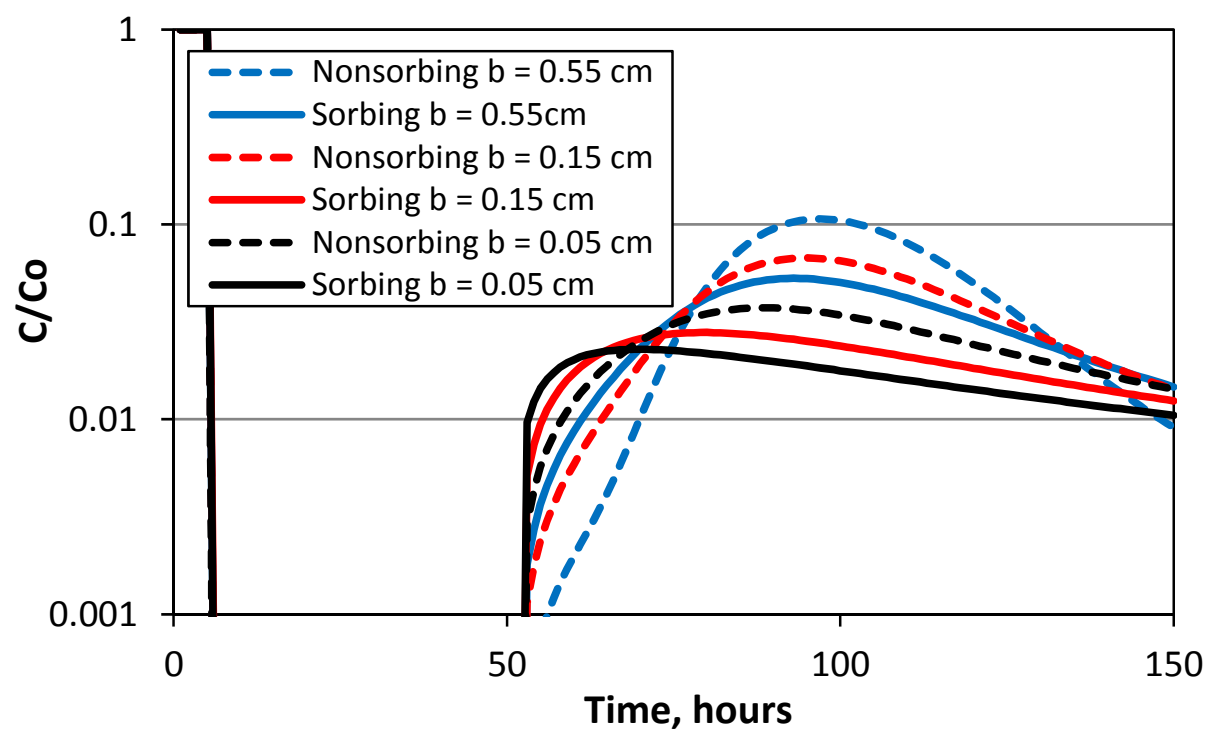

Figure 5.4. Simulated single-well breakthrough curves of nonsorbing and sorbing $\left(K_{d}=0.5 \mathrm{ml} / \mathrm{g}\right)$ tracers with the same diffusion coefficients (corresponding to a halide) as a function of fracture halfaperture in a $200^{\circ} \mathrm{C}$ reservoir. Tracers injected for $5 \mathrm{hr}$, followed by injection of tracer-free water for $45 \mathrm{hr}$, followed by a 2 -hr rest period before withdrawal. Matrix porosity $=0.05$.

When adsorption occurs to fracture walls or within thin fracture linings in single-well tracer tests, it does not provide the same delay in tracer arrival times relative to a conservative tracer that is observed in interwell tracer tests. In the case of a non-porous matrix, the retardation experienced during backflow effectively cancels out the retardation experienced during injection, and the net result is that the adsorbing tracer experiences the same mean arrival time as a conservative tracer, but with greater effective dispersion than a conservative tracer (i.e., an earlier arrival time and a longer tail). Figure 5.5 shows an example of this type of behavior in a simulated single-well tracer test in a system with no matrix diffusion.

As Figure 5.4 and Figure 5.5 imply, when both fracture and matrix adsorption take place at the same time it is very difficult to distinguish between the two processes and account for them separately because 
the delayed arrival time that is a hallmark of adsorption to fracture walls or linings in interwell tests does not occur in single-well tests. Thus, the use of adsorbing tracers to interrogate fracture half-apertures or $\mathrm{SA} / \mathrm{V}$ ratios in single-well tracer tests has more inherent uncertainty than it does in interwell tracer tests. Nevertheless, with careful characterization of the reservoir fracture and matrix properties, it should be possible to obtain estimates of fracture half-apertures from fast, linear, reversible adsorbing tracers in single-well tracer tests.

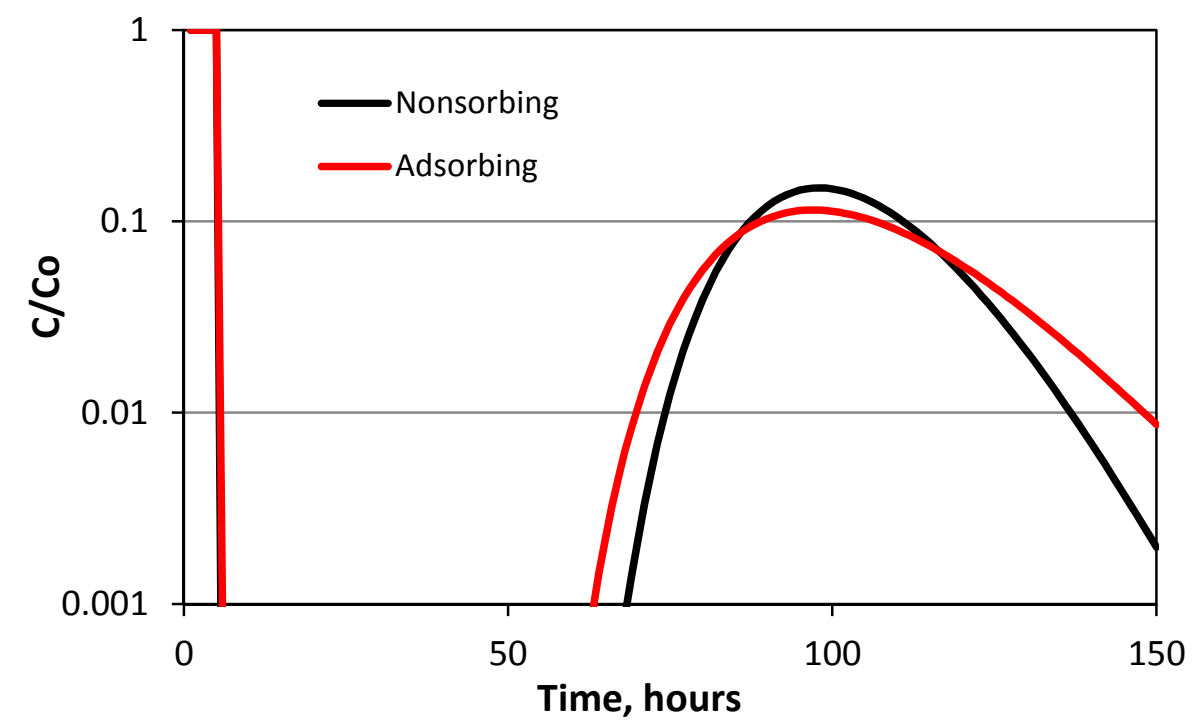

Figure 5.5. Simulated single-well breakthrough curves of nonsorbing and sorbing $\left(K_{d}=0.5 \mathrm{ml} / \mathrm{g}\right)$ tracers in a system with no matrix porosity. Tracers injected for $5 \mathrm{hr}$, followed by injection of tracer-free water for $45 \mathrm{hr}$, followed by a 2 -hr rest period before withdrawal.

\subsection{The Special Case of Cation-Exchanging Tracers in Single-Well Tracer Tests}

Cation-exchanging tracers represent a special case of adsorbing tracers in that they typically exhibit highly nonlinear adsorption behavior. In the case of interwell tracer tests, this nonlinear behavior does not cause cation-exchanging tracers to deviate significantly from the behavior of adsorbing tracers that have linear adsorption isotherms, because the high dilution factors in interwell tests tend to rapidly reduce cation tracer concentrations to the point where adsorption is well approximated by linearizing the lowconcentration end of an isotherm. The only exception to this behavior is when CECs in the reservoir are extremely low. Figure 5.6 shows an example of the dependence of a cation-exchanging tracer breakthrough curve on the fracture half-aperture or SA/V ratio in an interwell tracer test with the parameters of Table 4.1 and assuming cation exchange occurs only in the matrix, not on fracture surfaces.

The cation-exchanging tracer breakthrough curves of Figure 5.6 are qualitatively consistent with those of a fast, linear, reversibly adsorbing tracer in that they have the characteristics of a nonsorbing tracer with a much larger matrix diffusion coefficient than a conservative tracer (i.e., lower peak concentrations and longer tails than the conservative tracer). Because of the similarity in the behavior of cation-exchanging tracers and linearly adsorbing tracers in interwell tracer tests, the use of cationexchanging tracers in interwell tracer tests is not discussed further here. Also, cation-exchanging tracers 
are unlikely to be practical in interwell tracer tests because their relatively high detection limits and significant background concentrations in many geothermal reservoirs are not compatible with the large dilution factors observed in most interwell tracer tests.

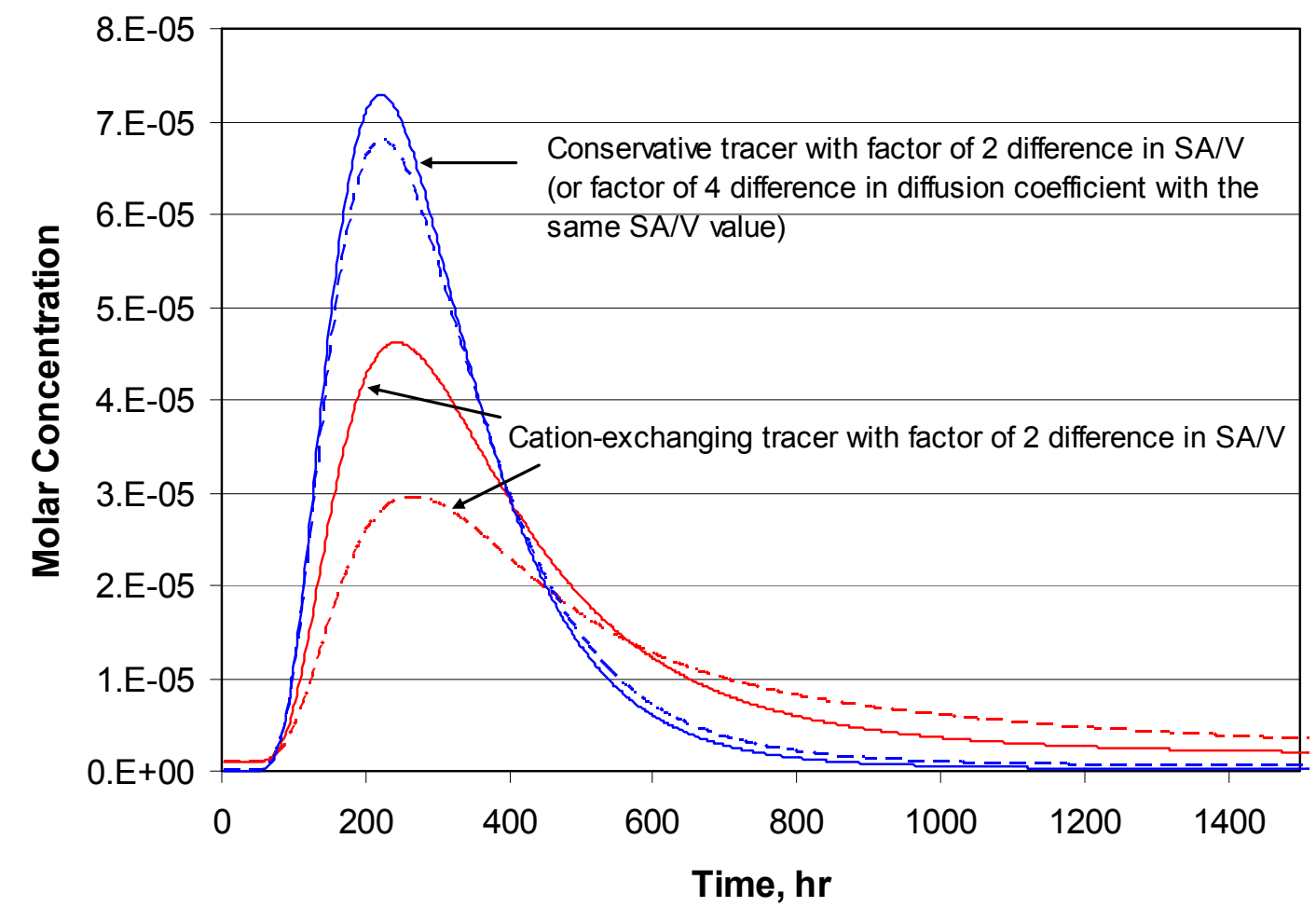

Figure 5.6. Simulated differences in conservative and cation-exchanging tracer breakthrough curves for $\mathrm{SA} / \mathrm{V}$ values of $10 \mathrm{~cm}^{-1}$ and $20 \mathrm{~cm}^{-1}$ (or fracture half-aperture values of 0.1 and $0.05 \mathrm{~cm}$,

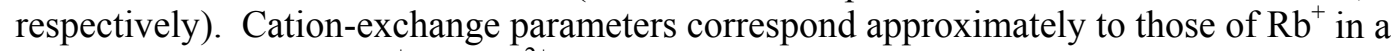
system dominated by $\mathrm{Na}^{+}$and $\mathrm{Ca}^{2+}$ (as resident cations). The molar concentrations and durations of the injection pulses were the same for all breakthrough curves, so the plotted molar concentrations are proportional to normalized concentrations.

In single-well tracer tests, cation-exchanging tracer concentrations can easily remain high enough that the use of these tracers is practical and their nonlinear adsorption behavior must be accounted for.

Figure 5.7 shows the simulated breakthrough curves of a relatively strongly exchanging cation in singlewell tracer tests in systems with three different fracture half-apertures that vary over approximately an order of magnitude. Also shown for comparison in Figure 5.7 are the responses of two conservative tracers with different diffusion coefficients and a linearly adsorbing tracer with a partition coefficient in the matrix that is set so that its adsorption is similar in magnitude to that of the cation-exchanging tracer. The other model parameters are listed in Table 5.1. 

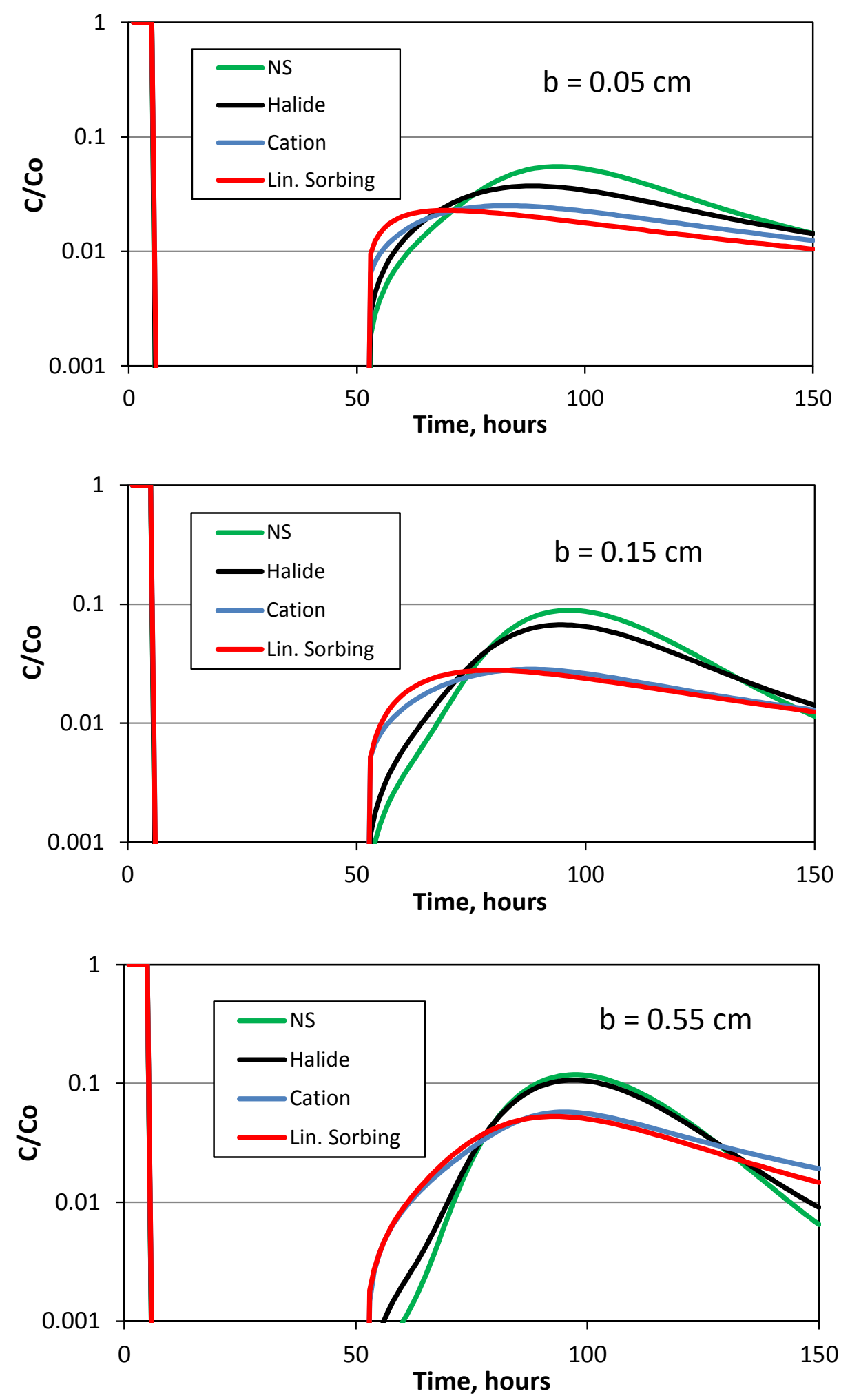

Figure 5.7. Simulated single-well breakthrough curves as a function of fracture half-aperture for a naphthalene sulfonate, a halide, a cation-exchanging tracer, and a linear reversibly adsorbing tracer after a tracer injection of $5 \mathrm{hr}$, injection of tracer-free water for $45 \mathrm{hr}$, and a 2 -hr rest period. Other model parameters are listed in Table 5.1. 
Table 5.1. Model parameters used in MULTRAN simulations of Figure 5.7 through Figure 5.9.

\begin{tabular}{ll}
\hline Initial temperature of reservoir & $200^{\circ} \mathrm{C}$ \\
Injection water temperature & $25^{\circ} \mathrm{C}$ \\
Fracture half-apertures & $0.05,0.15$, and $0.55 \mathrm{~cm}$ \\
Fracture spacing & $200 \mathrm{~cm}$ \\
Matrix porosity & $0.05(5 \%)$ \\
Matrix cation exchange capacity & $0.03 \mathrm{eq} / \mathrm{kg}$ (no exchange on fracture surfaces) \\
Selectivity coefficient, $K_{\text {Na/C }}$ & $\sim 0.1($ equivalent to that for $\mathrm{Li}$ in Newberry Crater system \\
& at $200^{\circ} \mathrm{C}$ from results of Section 6.2 .1$)$ \\
Matrix $K_{d}$ of linearly adsorbing tracer & $0.5 \mathrm{ml} / \mathrm{g}$ \\
Tracer matrix diffusion coefficients & $1 \times 10^{-6} \mathrm{~cm}^{2} / \mathrm{s}$ at $25^{\circ} \mathrm{C}$ for halide and linearly adsorbing \\
& tracer \\
& $2.5 \times 10^{-7} \mathrm{~cm}^{2} / \mathrm{s}$ at $25^{\circ} \mathrm{C}$ for naphthalene sulf. \\
& $6.7 \times 10^{-7} \mathrm{~cm}^{2} / \mathrm{s}$ at $25^{\circ} \mathrm{C}$ for cation \\
Rock and water properties (density and thermal) & Corresponding to basalt and dilute water \\
\hline
\end{tabular}

The tracer breakthrough curves in Figure 5.7 show that under the simulated conditions the cationexchanging tracer behaves quite similarly to the linearly adsorbing tracer, but there are differences that are important to account for when the fracture half-aperture is varied. These differences would be more pronounced if the CEC were smaller or if the cation were assigned a larger selectivity coefficient (making it more likely to be displaced from the surface by other cations). Figure 5.8 shows the results of a simulated tracer test with a fracture half-aperture of $0.15 \mathrm{~cm}$ and all other parameters the same as those listed above except that the CEC in the matrix was reduced from 0.03 to $0.0003 \mathrm{eq} / \mathrm{kg}$. The cation breakthrough curve becomes higher in concentration relative to the 0.15 -cm half-aperture case in Figure 5.7 because of the decreased capacity of the matrix to adsorb cations.

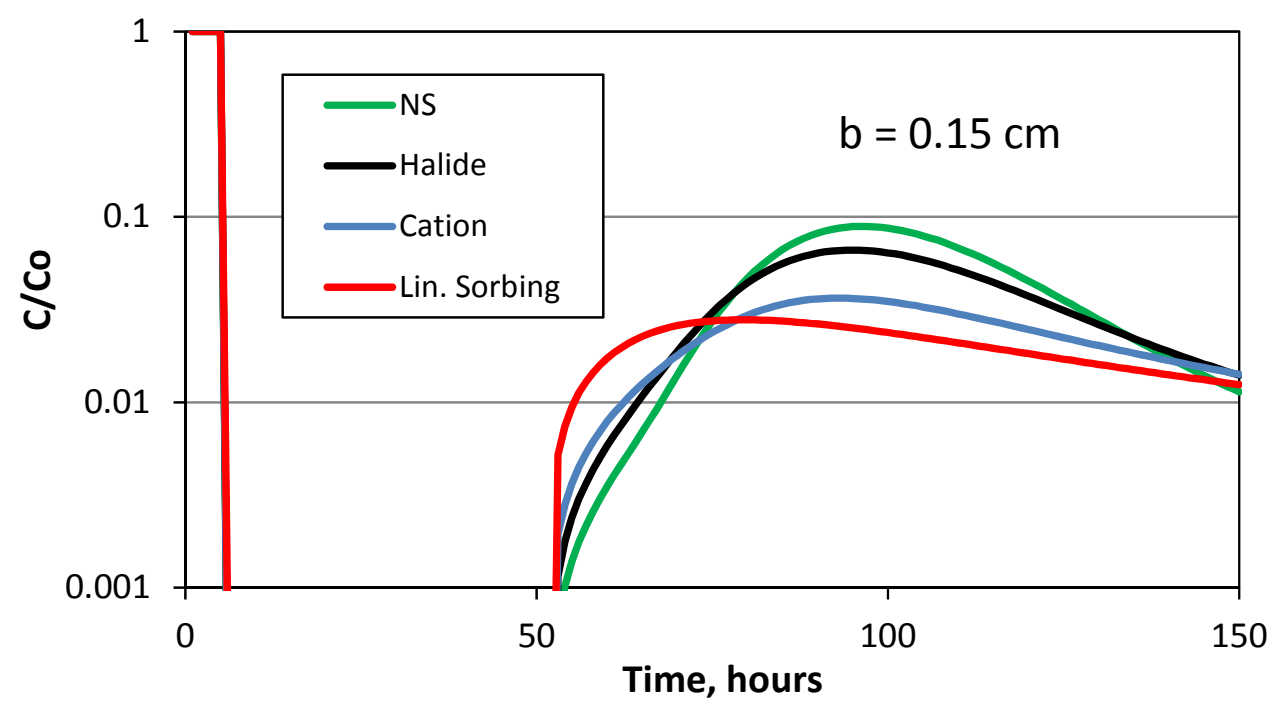

Figure 5.8. Simulated single-well breakthrough curves for a naphthalene sulfonate, a halide, a cationexchanging tracer, and a linear reversibly adsorbing tracer in a system with a fracture halfaperture of $0.15 \mathrm{~cm}$ (same as middle plot of Figure 5.7), but with a matrix CEC of $0.0003 \mathrm{eq} / \mathrm{kg}$. 
Figure 5.9 shows single-well tracer test breakthrough curves for a significantly different set of assumed model parameters than Figure 5.7 and Figure 5.8. In this case, the matrix is assumed to have a very low porosity $(0.00001$ or $0.001 \%)$ except for a very thin layer $(0.04 \mathrm{~cm}$ thick $)$ at the fracture wall, and even this layer is only assigned a porosity of 0.0012 or $0.12 \%$. However, this thin layer is also assigned a relatively large CEC of $0.046 \mathrm{eq} / \mathrm{kg}$. These layer properties correspond to the properties that provided a very good simultaneous match to four different lithium laboratory column transport experiments that were conducted at four different temperatures using crushed minerals that were selected to represent the fracture mineralogy at the Newberry Crater, Oregon EGS site, discussed in Section 6.2.1 (also see Table 6.8). The assumed temperature in all the simulations was $275^{\circ} \mathrm{C}$, which is the approximate temperature at the depth of an interval that lithium ion was injected into at Newberry Crater during EGS stimulations in November and December 2012.

In Figure 5.9, the ability to use conservative tracers with different diffusion coefficients to distinguish between different fracture half-apertures is effectively lost because of the extremely low matrix porosities in the simulated system(s). The conservative tracer breakthrough curves effectively fall on top of each other despite the order-of-magnitude range in fracture half-apertures in the simulations. However, there are significant differences in the adsorbing tracer breakthrough curves for the different fracture halfapertures, implying that the adsorbing tracers could still be used to provide estimates of half-apertures in single-well tracer tests in such a system even though the conservative tracers fail to provide this information. The linearly adsorbing non-cation-exchanging tracer in Figure 5.9 is assigned the same matrix partition coefficient as in the simulations of Figure 5.7 and Figure 5.8.

Figure 5.10 shows the results of simulations conducted at four different temperatures ranging from $50^{\circ} \mathrm{C}$ to $275^{\circ} \mathrm{C}$ using the same physical system parameters and tracer testing parameters (50-hr injection, 2-hr rest) as in Figure 5.9, but with the fracture half-aperture fixed to its smallest value in Figure 5.9. The differences in the simulated breakthrough curves in this case are a result of the temperature dependence of the matrix diffusion coefficients of the tracers as well as the temperature dependence of the cationexchange selectivity coefficient $K_{N a-L i}$ for lithium, which is deduced from the laboratory column experiments discussed in Section 6.2.1. Both temperature effects tend to increase the separation between the conservative tracer and adorbing tracer breakthrough curves at higher temperatures. Note that the matrix partition coefficient of the linearly adsorbing non-cation-exchanging tracer was assumed to have no temperature dependence, so the differences in the breakthrough curve of this tracer as a function of temperature purely reflect the influence of the temperature dependence of the diffusion coefficient. Note also that the breakthrough curves of the conservative tracers with different diffusion coefficients were identical at the different temperatures despite the significant differences in the diffusion coefficients as a function of temperature. These results show the importance of accounting for temperature when using adsorbing tracers to interrogate surface-area-to-volume ratios or fracture half-apertures in single-well tracer tests.

Figure 5.11 shows that the tracer breakthrough curves obtained at $275^{\circ} \mathrm{C}$ for the simulated conditions of Figure 5.10 but with significantly different rest periods $(2 \mathrm{hr} v \mathrm{vs} .50 \mathrm{hr})$ are quite similar. This result is different than what is predicted for a system of significant matrix porosity where all tracers are expected to exhibit significantly lower peak concentrations and longer tails after a longer rest period because of the additional matrix diffusion that occurs during the rest period. The result of Figure 5.11 demonstrates that in a system of very low matrix porosity where there is no observed separation in the breakthrough curves of conservative tracers with different diffusion coefficients, estimates of fracture half-apertures from adsorbing tracers are relatively insensitive to rest periods. 

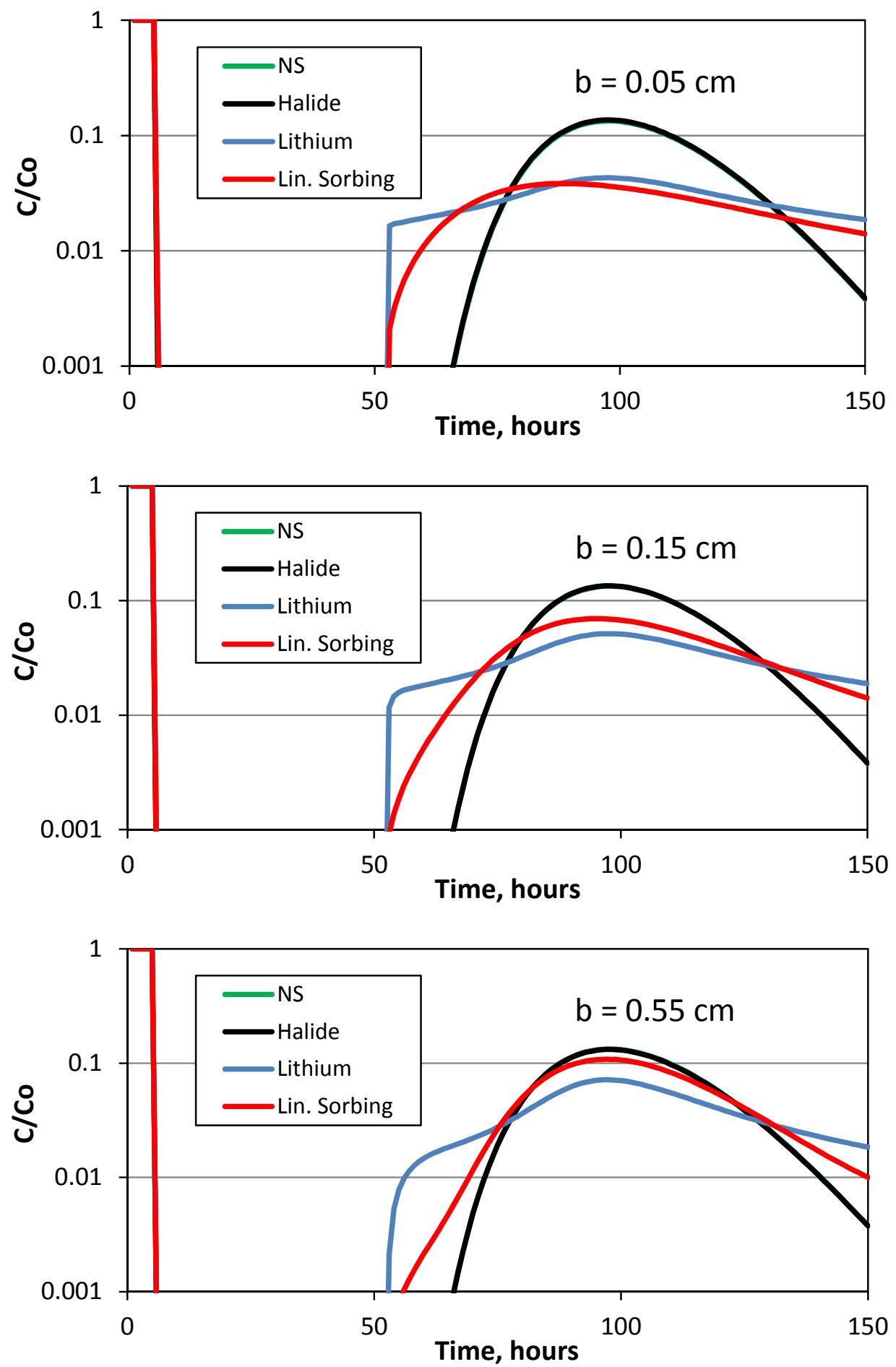

Figure 5.9. Simulated single-well breakthrough curves as a function of fracture half-aperture for a naphthalene sulfonate, a halide, a cation-exchanging tracer, and a linear reversibly adsorbing tracer in a system with matrix diffusion and cation exchange parameters similar to those measured in the Newberry Crater laboratory column experiments of Section 6.2.1.

Simulated tracer injection of $5 \mathrm{hr}$, followed by injection of tracer-free water for $45 \mathrm{hr}$, and then a 2-hr rest period. Matrix diffusion and cation exchange parameters are discussed in the text and listed in Table 6.8. 

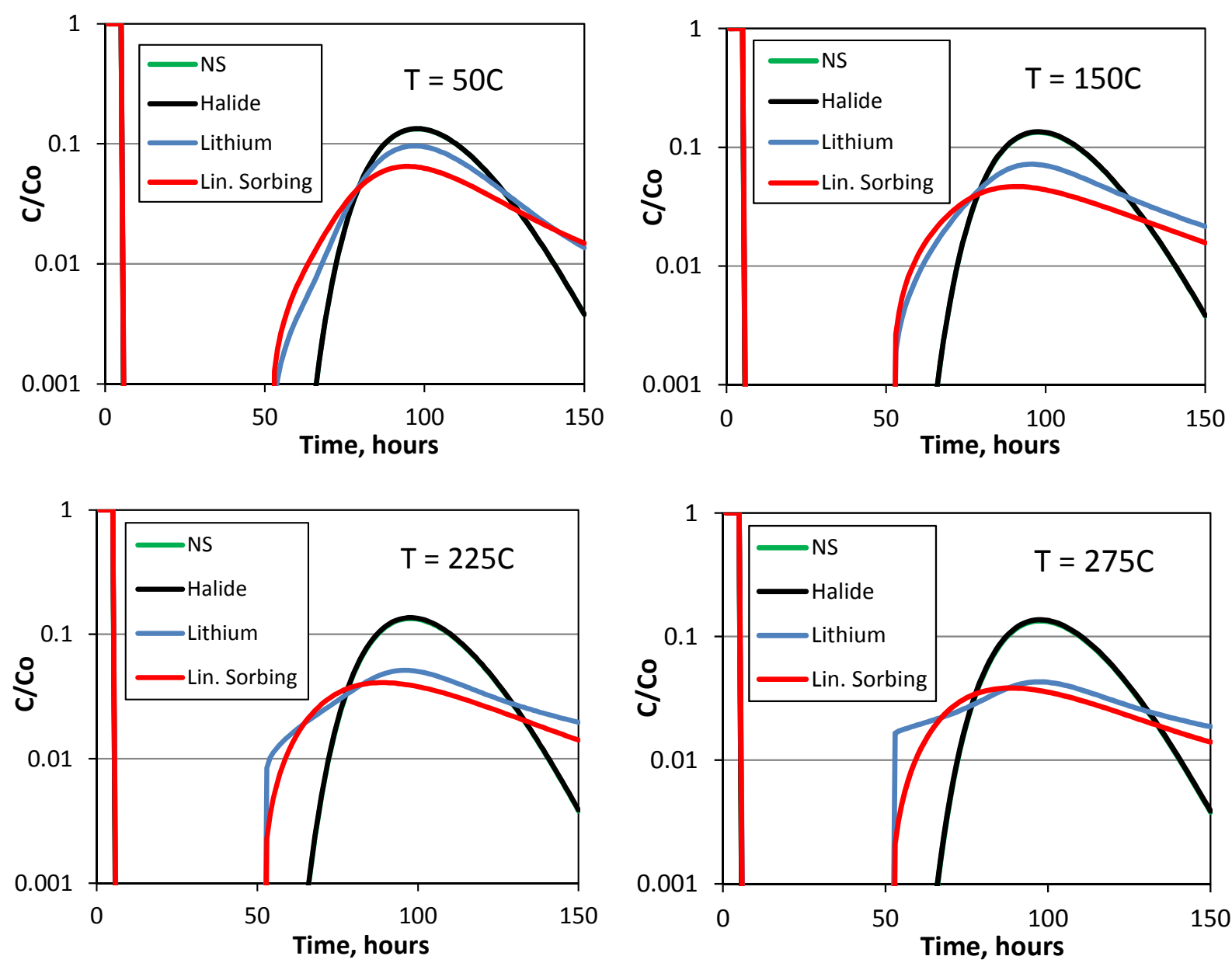

Figure 5.10. Simulated single-well breakthrough curves as a function of reservoir temperature for a naphthalene sulfonate, a halide, a cation-exchanging tracer, and a linear reversibly adsorbing tracer in a system with matrix diffusion and cation exchange parameters similar to those measured in the Newberry Crater laboratory column experiments of Section 6.2.1 (fracture half-aperture $=0.05 \mathrm{~cm}$ ). Simulated tracer injection of $5 \mathrm{hr}$, followed by injection of tracer-free water for $45 \mathrm{hr}$, and then a 2-hr rest period. Matrix diffusion and cationexchange parameters are discussed in text and listed in Table 6.8 .

Another testing parameter that can be varied in single-well tracer tests is the duration of the injection pulse, and in particular, whether the tracers are injected as a finite pulse (potentially at different times before the end of the injection) or continuously at a constant concentration throughout the injection period. Figure 5.12 shows the differences in tracer breakthrough curves for the injection of the same tracer masses in a finite pulse and in a continuous injection in a system having a matrix porosity of 0.05 (identical to the system simulated for Figure 5.7 and Figure 5.8). A similar comparison is shown in Section 6.1 for the case of a linearly adsorbing tracer that also thermally decays using the TOUGHREACT code and the parameters deduced for the Soda Lake, Nevada system. Figure 5.13 shows the differences in breakthrough curves for the injection of the same tracer masses in a finite pulse and a continuous injection in a system having the properties simulated in Figure 5.9 with a fracture halfaperture of $0.55 \mathrm{~cm}$ but with the fractures assumed to be filled with a weakly adsorbing fill material that gives them a $40 \%$ internal porosity. The CEC of the fill material in this case was assigned a value of 
$0.0011 \mathrm{eq} / \mathrm{kg}$. Here it is apparent that the continuous tracer injection may provide certain advantages over the pulse injection in that the slopes of the tails of the breakthrough curves might serve as more reliable indicators of fracture properties than the peak tracer concentrations after a finite pulse. The tail slopes after a continuous injection will not be nearly as dependent on accurate normalization of the tracer concentrations as the peak concentrations after a finite pulse.
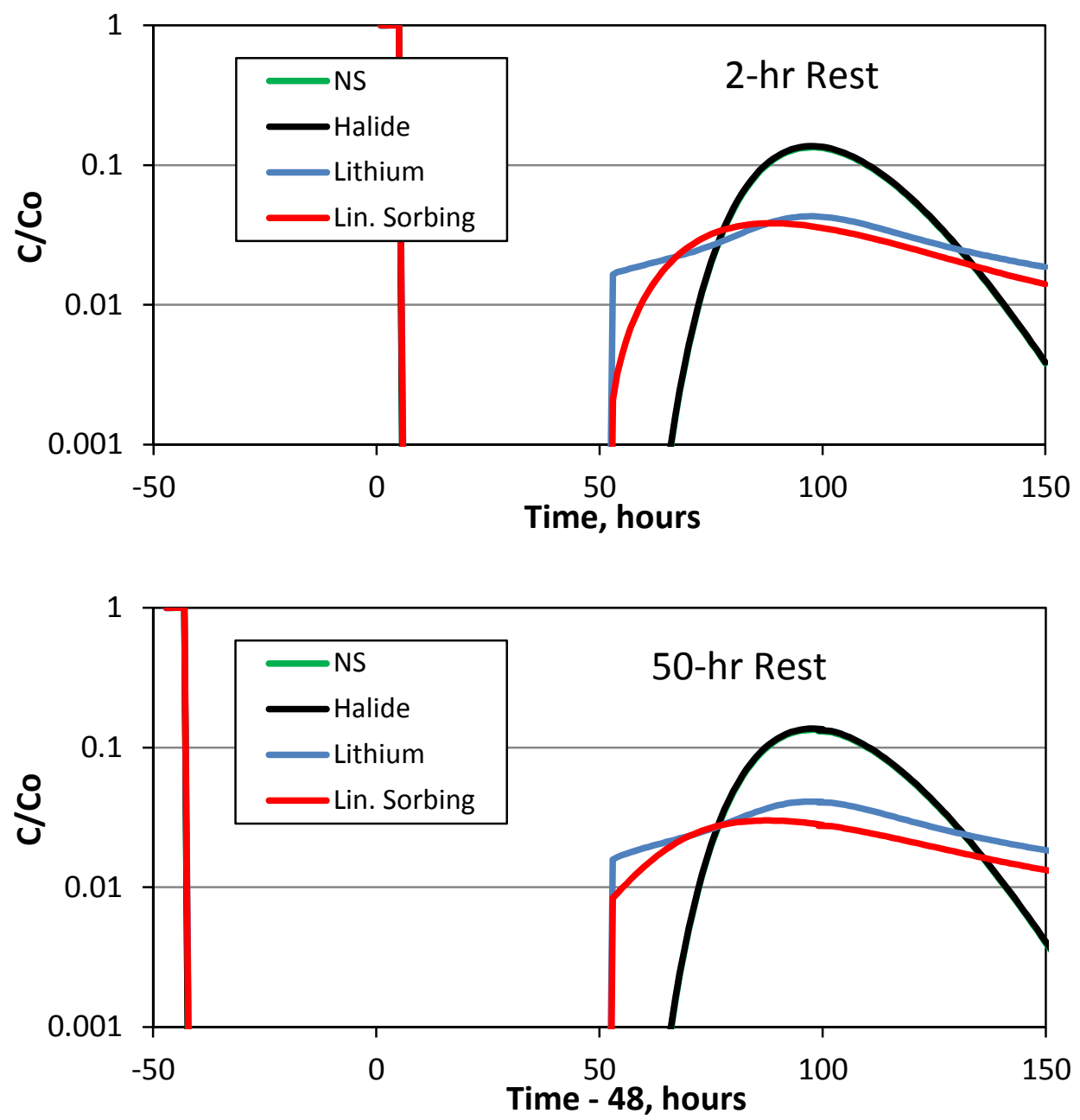

Figure 5.11. Simulated single-well breakthrough curves as a function of rest period for a naphthalene sulfonate, a halide, a cation-exchanging tracer, and a linear reversibly adsorbing tracer in a system with matrix diffusion and cation-exchange parameters similar to those measured in the Newberry Crater laboratory column experiments of Section 6.2.1 (fracture halfaperture $=0.05 \mathrm{~cm}$, temperature $=275^{\circ} \mathrm{C}$ ). Simulated tracer injection of $5 \mathrm{hr}$, followed by injection of tracer-free water for $45 \mathrm{hr}$, and then a 2 -hr or $50-\mathrm{hr}$ rest period. Matrix diffusion and cation-exchange parameters are discussed in the text and listed in Table 6.8.

All of the cases considered in this section, and the corresponding breakthrough curves presented in the figures, serve to only scratch the surface of the many possibilities of cation-exchanging tracer responses (and adsorbing tracer responses in general) that could occur in single-well injection withdrawal tests. The wide range of potential tracer adsorption or cation-exchange parameters, including the adsorption/exchange capacity of the rock, result in an essentially infinite range of possibilities. However, 
the cases presented here should serve to illustrate some general trends that can be expected and some advantages as well as limitations of using adsorbing tracers in single-well tracer tests for surface area interrogation. The important take-home message related to cation-exchanging tracers and linearly adsorbing tracers is that the diffusion and adsorption behavior of the tracers should be carefully characterized in the specific system in which they will be applied to obtain the best possible estimates of fracture half-apertures or $\mathrm{SA} / \mathrm{V}$ ratios.
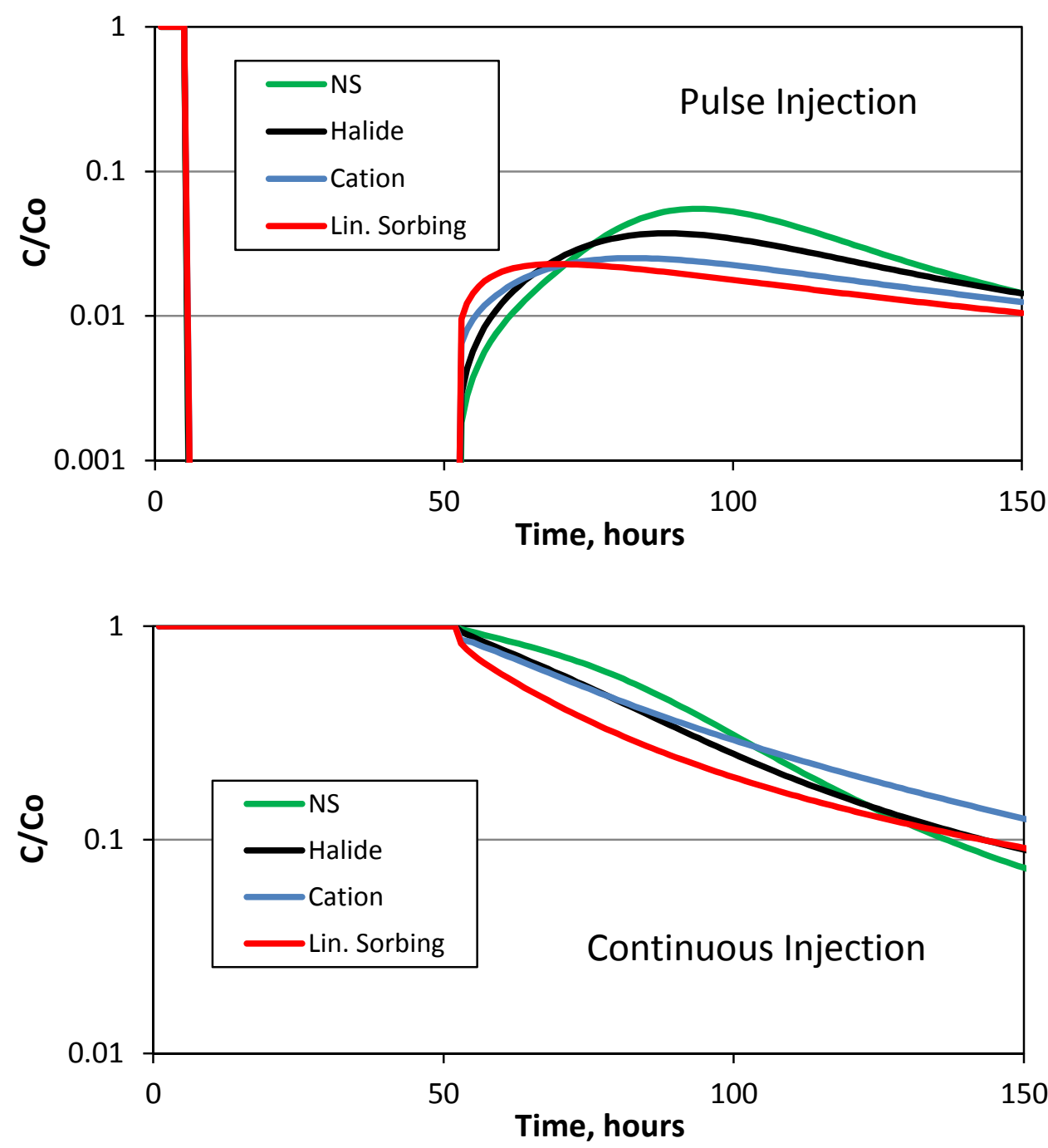

Figure 5.12. Simulated single-well breakthrough curves for the $b=0.05 \mathrm{~cm}$ case of Figure 5.7 with a pulse injection (identical to top of Figure 5.7) and with a continuous injection over the entire $50 \mathrm{hr}$ of injection. Other model parameters are listed in Table 5.1. 

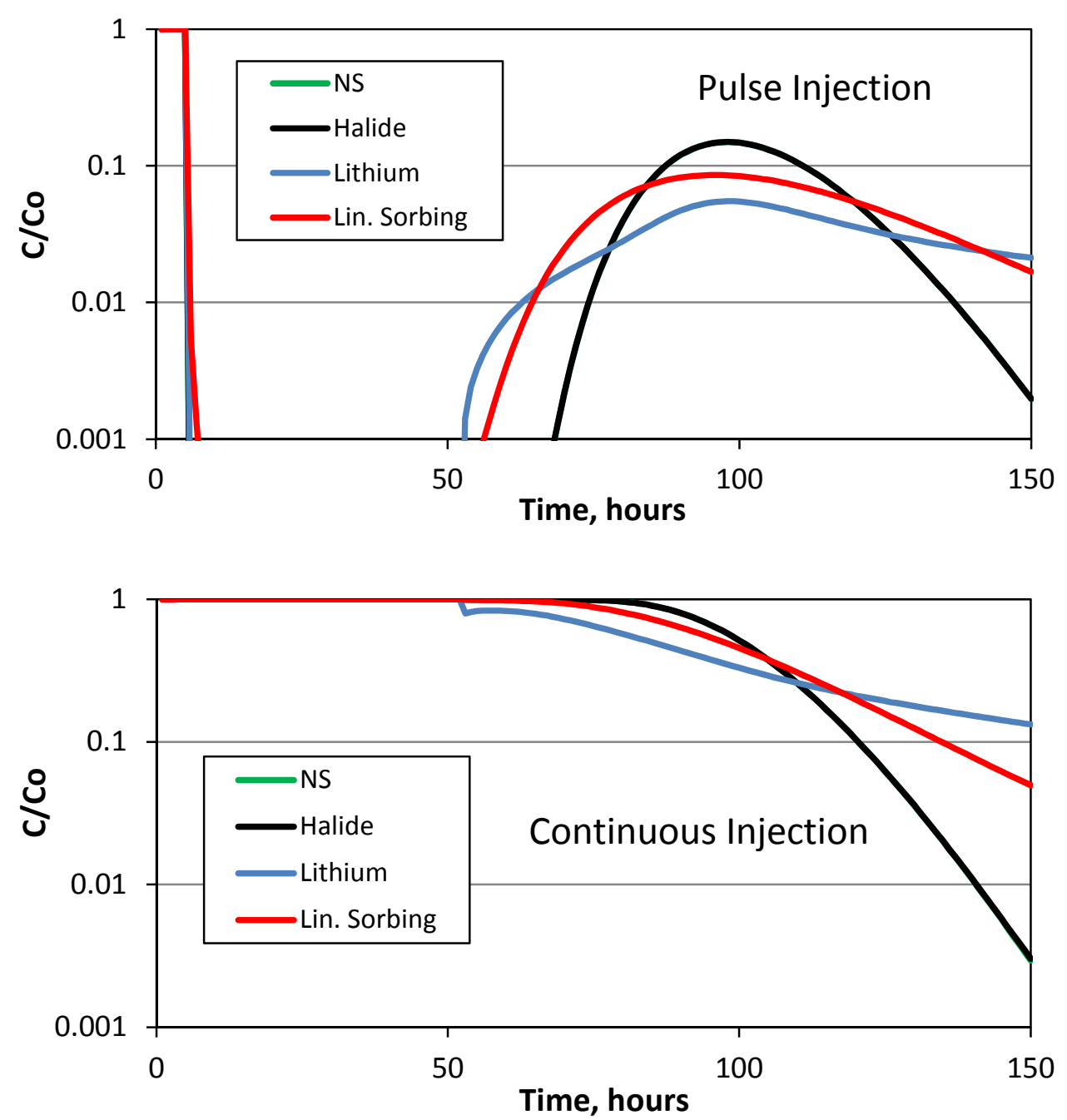

Figure 5.13. Simulated single-well breakthrough curves for the $b=0.55 \mathrm{~cm}$ case of Figure 5.9 but with the addition of fracture fill material that has a cation-exchange capacity of $0.0011 \mathrm{eq} / \mathrm{kg}$, a $K_{d}$ value of $0.5 \mathrm{ml} / \mathrm{g}$, and causes an internal fracture porosity of 0.4 . Shown are a comparison of a pulse injection and a continuous injection over the entire $50 \mathrm{hr}$ of the injection period. Other model parameters are listed in Table 6.8. Note minor differences in pulse injection case here and the bottom plot of Figure 5.9, which are due to the additional influence of the fracture fill material here. 


\subsection{Field Applications}

This study looked at using modeling for analysis of reactive and conservative tracers for characterization of two geothermal sites. The first site, Soda Lake, Nevada, is an operating geothermal field with naturally occurring fractures where reactive and conservative tracer tests were conducted in 2011. The results of these tracer tests, including breakthrough curves of the tracers, were provided to the project team by EGI for fitting with different models to estimate site, fracture, and tracer parameters. The second field site, Newberry Crater, Oregon, is an EGS demonstration site operated by AltaRock Energy Inc. For the Newberry Crater site, the project team was responsible for developing a preliminary design of the tracer tests planned for the site during the three stimulations. The project team also was responsible for conducting the tracer tests during the EGS stimulations in November and December 2012. These studies are described below.

\subsection{Soda Lake, Nevada Tracer Test Simulations}

The Soda Lake, Nevada site is an operating geothermal field (see Figure 6.1). While it is not an EGS site, it is dominated by fracture flow (see Echols et al. 2011). Conservative and sorbing tracer tests were conducted at the site in the fall of 2011 and provided the opportunity for developing and testing the tools and modeling approach of this study. Rose et al. (2012) describe the tracer test involving both a thermally stable conservative tracer (1,6-naphthalene disulfonate, or 1,6-NDS) and a thermally degrading sorbing tracer (Safranin-T, or Saf-T) conducted in a geothermal field at Soda Lake (see Figure 6.1). Below we provide semi-analytical and numerical modeling interpretations of the tracer breakthrough curves from that experiment, and we use the deduced parameters in numerical simulations of single-well injection/withdrawal tracer tests to show how the tracers could be used to estimate fracture surface area in such tests. This analysis was also partially published by Reimus et al. (2012) and Rose et al. (2012).

Details of the Soda Lake tracer tests are provided in Rose et al. 2012. The tracers were injected into Well 45A-33 on August 30, 2011. Tracer masses of $50 \mathrm{~kg}$ 1,6-NDS and $90 \mathrm{~kg}$ Saf-T were mixed in concentrated solutions in a small tank at the site in injected. The concentrated 1,6-NDS solution was injected over a period of $1 / 2 \mathrm{hr}$ followed by the injection of the concentrated Saf-T solution that was injected over a period of $3 \mathrm{hr}$ because of operational difficulties. A continuous injection rate of $\sim 800 \mathrm{gpm}$ was maintained during the test (see Figure 6.1). Production from Well 32-33 was $\sim 885$ gpm with the production water (along with water produced from other wells in the field) re-injected into Well 45A-33 during the subsequent $28 \mathrm{~d}$. Arrhenius parameters for the thermal degradation of Saf-T were determined in laboratory studies conducted at EGI (see Figure 6.2).

The semi-analytical and numerical models used to fit the 1,6-NDS and Saf-T breakthrough curves measured in Well 32-33 from the multi-well test are described below. Simulated single-well injection/withdrawal tests using the derived site and tracer parameters are also described below to investigate possible test responses for potential application in characterization of site fractures. 

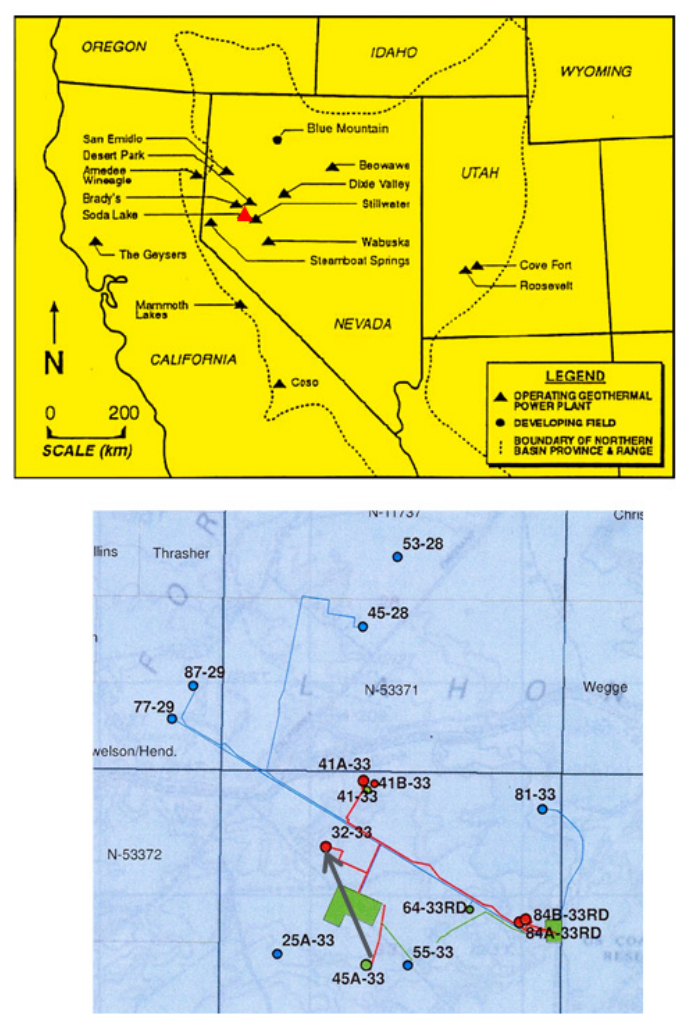

Figure 6.1. Soda Lake, Nevada, tracer test site location and well schematic.

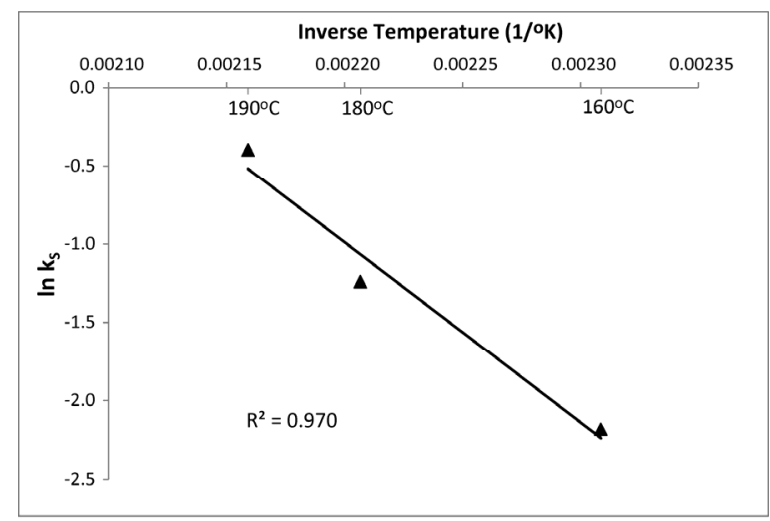

$$
\ln k_{S}=24.3-\frac{11500}{T}
$$

Figure 6.2. Arrhenius parameters for the thermal degradation of Saf-T determined from laboratory studies at EGI (from Rose et al. 2012) $\left(k_{\mathrm{s}}\right.$ units $\left.=1 / \mathrm{d}\right)$. 


\subsubsection{Semi-Analytical Model Analysis}

The semi-analytical RELAP model (see Chapter 4.0), which was developed to interpret multiple tracer breakthrough curves in interwell tracer tests in either single- or dual-porosity media (Reimus et al. 2003), was used to provide an initial interpretation of the Soda Lake tracer test. RELAP uses a relatively simple retardation approach to model reactive transport. Laboratory testing with Saf-T (Leecaster et al. 2012) suggested that this approach should be adequate for modeling the transport of this reactive tracer. The thermal decay corrections described by Rose et al. (2012), in which an average reservoir temperature of $180^{\circ} \mathrm{C}$ was assumed, were applied to the Saf-T field data. The concentrations of both the 1,6-NDS and the Saf-T were normalized by dividing their observed concentrations in the production well (decay corrected in the case of Saf-T) by their injection masses prior to analysis by RELAP. The resulting tracer breakthrough curves are shown in Figure 6.3.

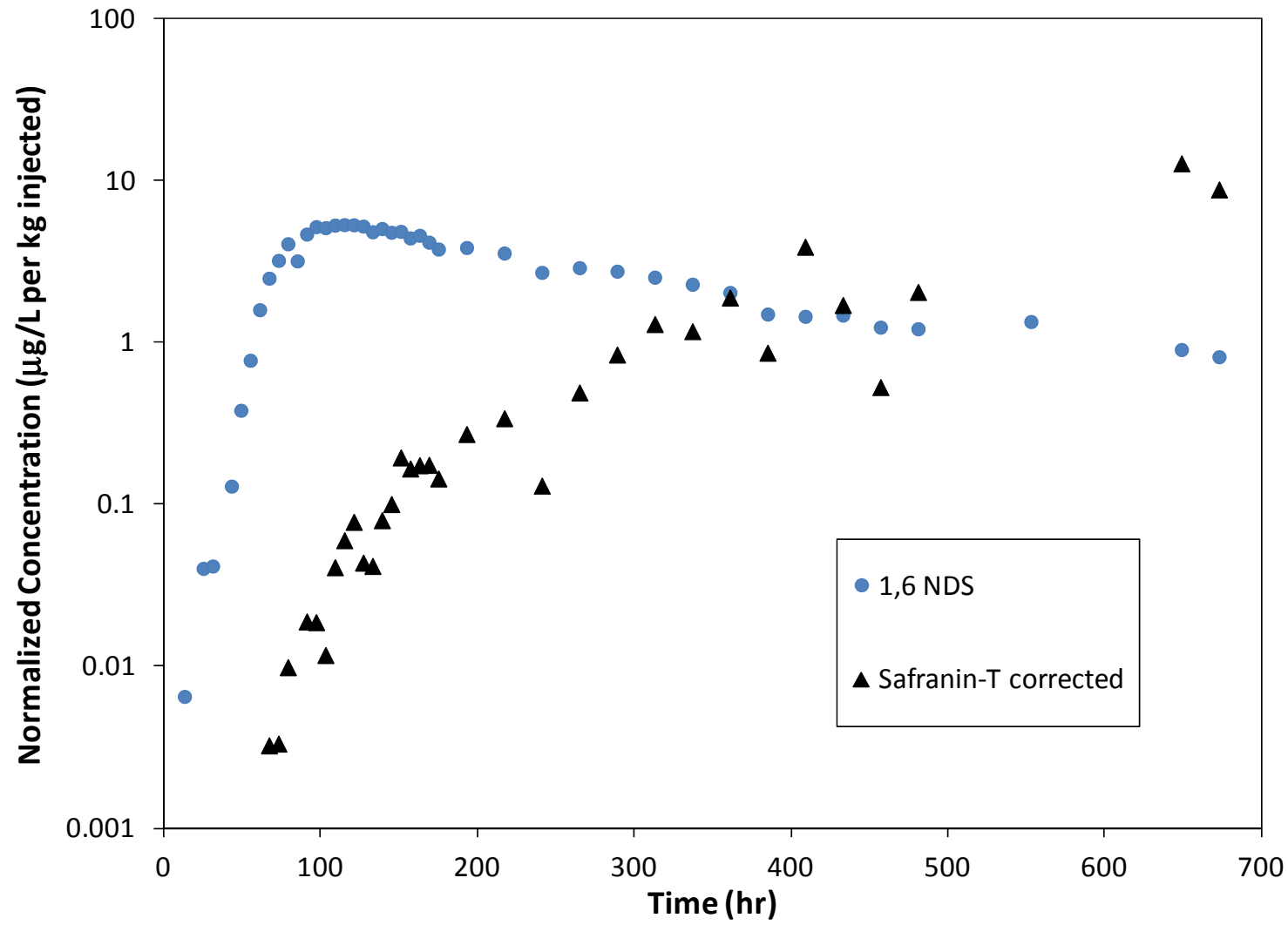

Figure 6.3. Normalized breakthrough curves of 1,6-NDS and Saf-T in the Soda Lake tracer test. Saf-T concentrations are decay-corrected assuming a reservoir temperature of $180^{\circ} \mathrm{C}$ and using the Arrhenius decay parameters of Rose et al. (2012).

The 1,6-NDS curve breakthrough was first matched by adjusting the mean residence time and Peclet number (travel distance divided by longitudinal dispersivity) assuming both a single-porosity system (no matrix diffusion) and a dual-porosity system (Figure 6.4). The tracer mass assumed to be participating in the test was also treated as an adjustable parameter, because the overall 1,6-NDS recovery was on the order of $30 \%$ when the test concluded. It was also assumed that $26.5 \%$ of any tracer mass recovered was recirculated to the injection well, reflecting reinjection and production flow rates $(800 \mathrm{gpm}$ and $885 \mathrm{gpm}$, 
respectively) as well as dilution of the production water with untraced water from other wells prior to reinjection. RELAP can robustly account for recirculated tracer mass.

Two dual-porosity cases were simulated: One with a nominally high and one with a nominally low amount of matrix diffusion (using diffusive mass transfer coefficients, $\frac{\emptyset}{b} \sqrt{D_{m}}$, equal to $1 \mathrm{e}-3 \mathrm{~s}^{-1 / 2}$ and $1 \mathrm{e}-4 \mathrm{~s}^{-1 / 2}$, respectively; where $\phi=$ matrix porosity, $b=$ average fracture half-aperture, $\mathrm{cm}$, and $D_{m}=$ matrix diffusion coefficient, $\mathrm{cm}^{2} / \mathrm{s}$ ). It can be shown that it is generally possible to fit single conservative tracer breakthrough curves almost equally well assuming a wide range of matrix diffusion mass transfer coefficients, including no matrix diffusion. The only reliable way to reduce the uncertainty in the assumed value of $\frac{\emptyset}{b} \sqrt{D_{m}}$ is to use two different conservative tracers with significantly different diffusion coefficients, but because this was not done at Soda Lake, we simply did sensitivity calculations over a relatively wide range.

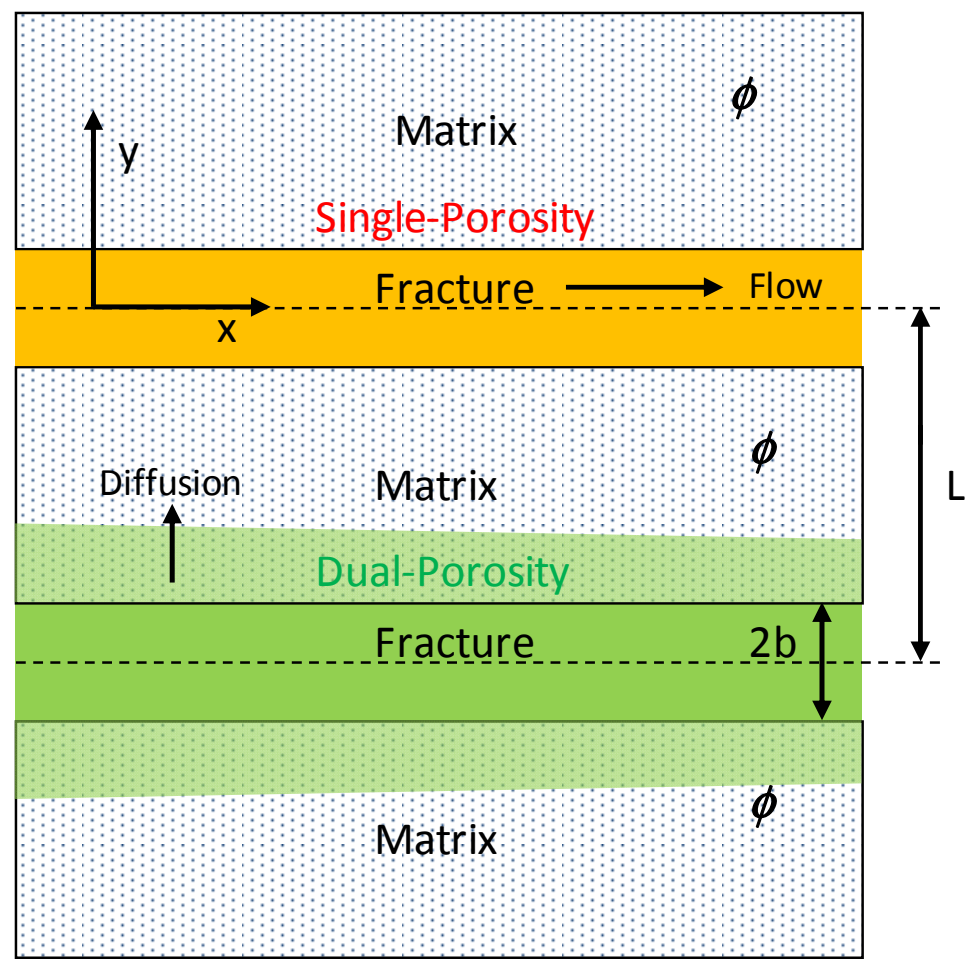

Figure 6.4. Schematic of single-porosity (top) and dual-porosity (bottom) conceptual models.

For perspective, if we assume a relatively high matrix porosity of 0.1 and a conservatively high matrix diffusion coefficient of $4 \mathrm{e}-6 \mathrm{~cm}^{2} / \mathrm{s}, \frac{\emptyset}{b} \sqrt{D_{m}}=1 \mathrm{e}-3 \mathrm{~s}^{-1 / 2}$ corresponds to an average fracture halfaperture, $\mathrm{b}$, of $0.2 \mathrm{~cm}$ and $\frac{\emptyset}{b} \sqrt{D_{m}}=1 \mathrm{e}-4 \mathrm{~s}^{-1 / 2}$ corresponds to an average fracture half-aperture of $2 \mathrm{~cm}$. If the matrix porosity were lowered to 0.01 , which is probably more realistic for Soda Lake, the fracture half-aperture would decrease to $0.02 \mathrm{~cm}$ and $0.2 \mathrm{~cm}$, respectively (for a matrix diffusion coefficient of $4 \mathrm{e}-6 \mathrm{~cm}^{2} / \mathrm{s}$ ). Given that average fracture half-apertures in flowing pathways at Soda Lake are likely to be greater than $0.02 \mathrm{~cm}$ to accommodate the relatively high injection and production flow rates without excessive pressure drop, $\frac{\emptyset}{b} \sqrt{D_{m}}=1 \mathrm{e}-3 \mathrm{~s}^{-1 / 2}$ would seem to be a reasonable conservative upper bound for 
this lumped parameter. On the other hand, a matrix porosity of 0.005 and a matrix diffusion coefficient of $1 \mathrm{e}-7 \mathrm{~cm}^{2} / \mathrm{s}$ would correspond to a fracture half-aperture of $0.016 \mathrm{~cm}$ if $\frac{\emptyset}{b} \sqrt{D_{m}}=1 \mathrm{e}-4 \mathrm{~s}^{-1 / 2}$, so $1 \mathrm{e}-4 \mathrm{~s}^{-1 / 2}$ is probably not a reasonable lower bound for $\frac{\emptyset}{b} \sqrt{D_{m}}$. Rather, the assumption of no matrix diffusion at all (i.e., $\frac{\emptyset}{b} \sqrt{D_{m}}=0$; a single-porosity system) serves as a good lower bound, and $\frac{\emptyset}{b} \sqrt{D_{m}}=1 \mathrm{e}-4 \mathrm{~s}^{-1 / 2}$ can be considered a mid-case.

After obtaining reasonable matches to the 1,6-NDS breakthrough curve for each of three cases discussed above (high, low, and no matrix diffusion), the best-fitting parameters (i.e., mean residence time, Peclet number, and mass fraction) were assumed to apply to the corrected Saf-T breakthrough data, and these data were fitted by adjusting either a retardation factor in the flowing porosity, $R_{f}$, and/or a retardation factor in the matrix porosity, $R_{m}$. Note that fracture retardation can be caused by sorption to the fracture walls or by sorption onto any material filling the fractures, such as granularized rock that has spalled off the fracture walls.

Fits to the 1,6-NDS and Saf-T breakthrough curves are shown in Figure 6.5 for the three different cases. It is apparent that reasonable fits to the data can be obtained in each case. Table 6.1 lists the bestfitting parameters for the different models. Table 6.2, Table 6.3, and Table 6.4 show combinations of fracture and matrix retardation factors and the corresponding sums of squares differences between measured and modeled log Saf-T concentrations for the different conceptual models. Sums of squares differences of log concentrations were minimized because the decay-corrected Saf-T concentrations span approximately 3 orders of magnitude, and the fitting of non-log-transformed data was clearly biased by the highest concentrations late in the test. It was desirable to avoid this biasing because the late concentrations have the greatest uncertainty in thermal decay corrections, resulting in large fluctuations in non-transformed concentrations late in the test. In fact, the last three Saf-T data points were not included in the fitting exercise for this reason.

Table 6.3 and Table 6.4 (for the dual-porosity cases) show that the estimate of the fracture retardation factor is much better constrained by the data than the estimate of the matrix retardation factor.

Comparison of the sum of squares differences in Table 6.2, Table 6.3, and Table 6.4 also suggest that the single-porosity model with no matrix diffusion offered the best fits, and the dual-porosity model with less matrix diffusion offered better fits than the model with more matrix diffusion. However, as Figure 6.5 shows, the differences between the best-fitting models are very subtle.

These results clearly point to the dominant importance of fracture retardation over matrix retardation in fitting the Saf-T data. Unfortunately, deducing fracture SA/V ratios from fracture retardation factors is not straightforward. In the case of a smooth-walled, parallel-plate fracture, the following relationship holds (also listed after Equation (4.4)):

$$
R_{f}=1+\frac{S A}{V} K_{a}=1+\frac{1}{b} K_{a}
$$

where $\quad K_{a}=$ tracer partition coefficient per unit surface area, $\mathrm{cm}^{3} / \mathrm{cm}^{2}$

$S A=$ surface area, $\mathrm{cm}^{2}$

$V=$ volume, $\mathrm{cm}^{3}$

$b=V / S A=$ fracture half-aperture, $\mathrm{cm}$. 


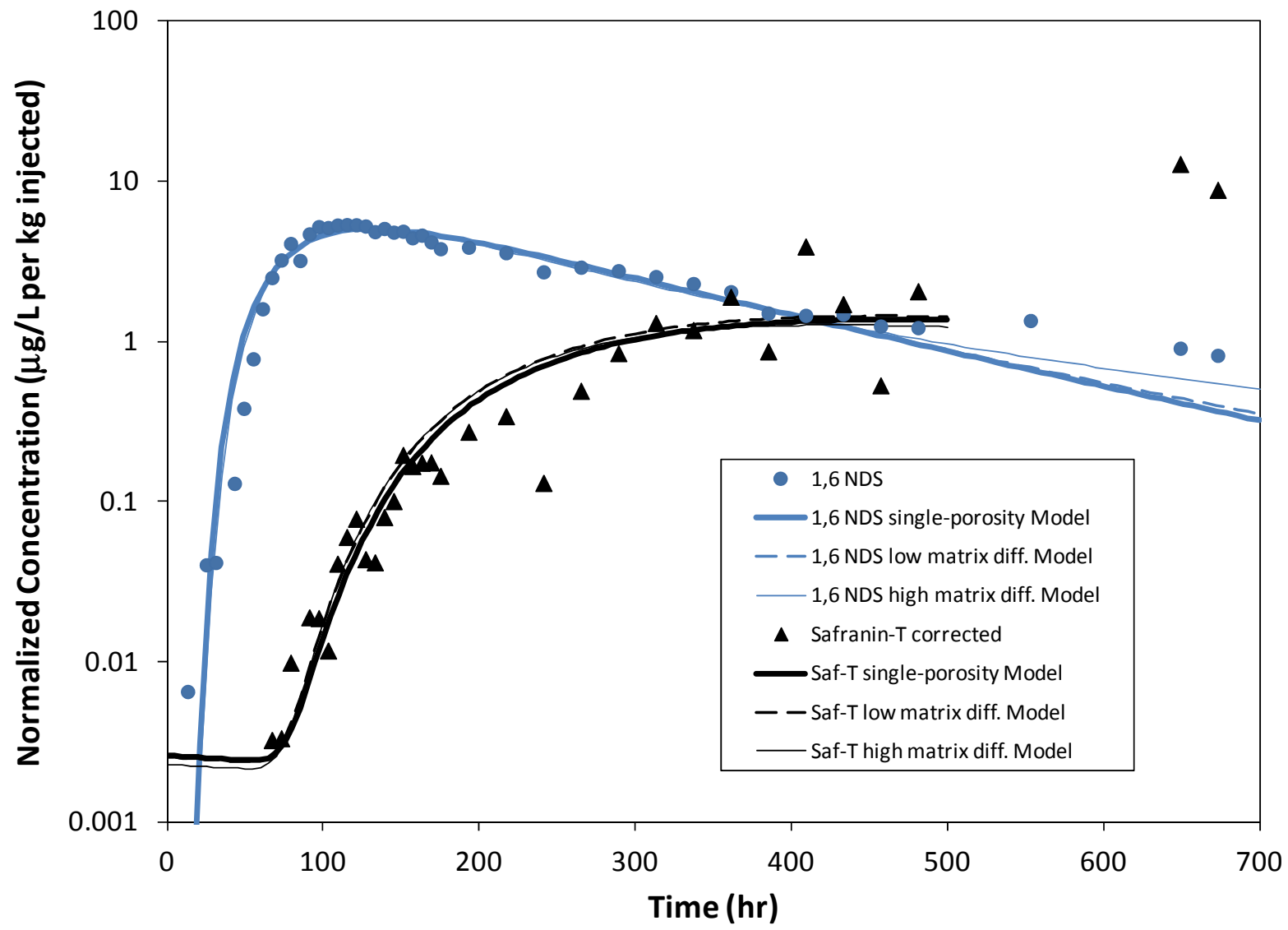

Figure 6.5. Single- and dual-porosity model fits to the 1,6-NDS and decay-corrected Saf-T breakthrough curves. Note that the last three Saf-T data points (beyond $500 \mathrm{hr}$ ) were not fitted.

Table 6.1. Best-fitting RELAP parameters for different conceptual models.

\begin{tabular}{lccc}
\hline \multicolumn{1}{c}{ Parameter } & No Matrix Diffusion & $\frac{\emptyset}{\boldsymbol{b}} \sqrt{\boldsymbol{D}_{\boldsymbol{m}}}=1 \mathrm{e}-4 \mathrm{~s}^{-1 / 2}$ & $\frac{\emptyset}{\boldsymbol{b}} \sqrt{\boldsymbol{D}_{\boldsymbol{m}}}=1 \mathrm{e}-3 \mathrm{~s}^{-1 / 2}$ \\
\hline Mean Res. Time, hr & 258 & 248 & 168 \\
Peclet Number & 4 & 4.2 & 7 \\
Tracer Mass Fraction & 0.2675 & 0.28 & 0.355 \\
Fracture Ret. Factor & 3.66 & $3.58-3.52$ & $3.34-3.14$ \\
Matrix Ret. Factor & $\mathrm{NA}$ & $1-4$ & $5-6.5$ \\
\hline
\end{tabular}


Table 6.2. Sensitivity of single-porosity model fits to fracture retardation factor. Gray rows indicate fits that are visually indistinguishable.

\begin{tabular}{cc}
\hline Fracture Ret. Factor & $\begin{array}{c}\text { Sum of Squares Differences } \\
\text { of Log Concentrations }\end{array}$ \\
\hline 3.58 & 2.031 \\
3.60 & 2.006 \\
3.62 & 1.988 \\
3.63 & 1.982 \\
3.64 & 1.977 \\
3.65 & 1.974 \\
3.66 & 1.972 \\
3.67 & 1.973 \\
3.68 & 1.975 \\
3.69 & 1.978 \\
3.70 & 1.984 \\
3.72 & 1.999 \\
3.74 & 2.021 \\
\hline
\end{tabular}

Table 6.3. Sensitivity of dual-porosity model fits with $\frac{\emptyset}{\boldsymbol{b}} \sqrt{\boldsymbol{D}_{\boldsymbol{m}}}=1 \mathrm{e}-4 \mathrm{~s}-1 / 2$ to fracture and matrix retardation factors. Gray rows indicate fits that are visually indistinguishable.

\begin{tabular}{ccc}
\hline Fracture Ret. Factor & Matrix Ret. Factor & $\begin{array}{c}\text { Sum of Squares Differences } \\
\text { of Log Concentrations }\end{array}$ \\
\hline 3.58 & 1 & 2.111 \\
3.56 & 1.5 & 2.126 \\
3.56 & 2 & 2.107 \\
3.54 & 2.5 & 2.130 \\
3.54 & 3 & 2.114 \\
3.52 & 4 & 2.129 \\
3.5 & 5 & 2.149 \\
3.48 & 7 & 2.152 \\
3.44 & 10 & 2.20 \\
3.4 & 15 & 2.23 \\
3.36 & 20 & 2.29 \\
3.3 & 30 & 2.37 \\
3.24 & 40 & 2.50 \\
3.16 & 60 & 2.64 \\
3.08 & 80 & 2.88 \\
\hline
\end{tabular}


Table 6.4. Sensitivity of dual-porosity model fits with $\frac{\emptyset}{b} \sqrt{D_{m}}=1$ e-3 s-1/2 to fracture and matrix retardation factors. Gray rows indicate fits that are visually indistinguishable.

\begin{tabular}{ccc}
\hline Fracture Ret. Factor & Matrix Ret. Factor & $\begin{array}{c}\text { Sum of Squares Differences } \\
\text { of Log Concentrations }\end{array}$ \\
\hline 4.2 & 1 & 4.483 \\
3.88 & 2 & 3.19 \\
3.66 & 3 & 2.60 \\
3.48 & 4 & 2.30 \\
3.4 & 4.5 & 2.22 \\
3.34 & 5 & 2.17 \\
3.26 & 5.5 & 2.16 \\
3.2 & 6 & 2.16 \\
3.14 & 6.5 & 2.17 \\
3.08 & 7 & 2.22 \\
2.98 & 8 & 2.30 \\
2.8 & 10 & 2.58 \\
2.64 & 12 & 2.95 \\
2.42 & 15 & 3.68 \\
\hline
\end{tabular}

Given an observed value of $R_{f}$ from a tracer test, a $K_{a}$ value must be known to estimate the fracture half-aperture, $b$. Leecaster et al. (2012) obtained estimates of $K_{a}$ values for Saf-T on Ottawa sand as a function of temperature by measuring $R_{f}$ values in column experiments in which the SA/V ratio was approximately known. Based on the reported sieve size fractions of the Ottawa sand used in the test(s) and assuming the sand grains were spherical, a geometric specific surface area of $\sim 100 \mathrm{~cm}^{2} / \mathrm{g}$ is calculated for the sand. Alternatively, gas adsorption surface areas (Brunauer-Emmet-Teller method) for Ottawa sand have been reported to be in the 900 to $1000 \mathrm{~cm}^{2} / \mathrm{g}$ range (Kibbey et al. 2007; Reimus et al. 2010). Using a range of specific surface areas from 100 to $1000 \mathrm{~cm}^{2} / \mathrm{g}$ along with the sand masses, pore volumes, and observed Saf-T retardation factor of $\sim 1.1$ at $160^{\circ} \mathrm{C}$ (the highest temperature tested) in the experiments of Leecaster et al. (2012), Sa-T $K_{a}$ values ranging from $2.3 \mathrm{e}-5 \mathrm{~cm}^{3} / \mathrm{cm}^{2}$ to $2.3 \mathrm{e}-4 \mathrm{~cm}^{3} / \mathrm{cm}^{2}$ are estimated from Equation (6.1). If these values are used in Equation (6.1) with the field-deduced $R_{f}$ value of 3.6, a range of fracture half-apertures from $8.8 \mathrm{e}-6 \mathrm{~cm}$ (for a sand surface area of $100 \mathrm{~cm}^{2} / \mathrm{g}$ ) to $8.8 \mathrm{e}-5 \mathrm{~cm}$ (for $1000 \mathrm{~cm}^{2} / \mathrm{g}$ ) is obtained. These half-apertures are deemed to be too small to support the injection and production flow rates at Soda Lake.

There are two problems with the above estimates of $b$. First, the SA/V ratio of a rough-walled fracture will undoubtedly be much larger than that of a parallel-plate fracture (the inherent assumption in Equation (6.1)). Second, the $K_{a}$ value onto volcanic tuff surfaces (the type of rock at Soda Lake) is likely to be different than that for quartz sand surfaces. However, Triay et al. (1997) and Sandia National Laboratories (2007) present arguments that, except for certain reactive species and certain minerals such as iron oxides and zeolites, $K_{a}$ values should be quite similar for many different rock surfaces. Indeed, they found that the mass-based partition coefficients, or $K_{d}$ values $\left(\mathrm{cm}^{3} / \mathrm{g}\right)$, of many radionuclides were roughly proportional to the specific surface area of different Yucca Mountain, Nevada tuffs, suggesting that although the $K_{d}$ values were often quite different, the $K_{a}$ values were similar. A typical specific surface area measured by gas adsorption for Yucca Mountain devitrified tuffs is $\sim 3.5 \mathrm{~m}^{2} / \mathrm{g}$, or 
$35,000 \mathrm{~cm}^{2} / \mathrm{g}$ (Triay et al. 1997), whereas for zeolitic tuffs the average is almost an order of magnitude higher at $\sim 28 \mathrm{~m}^{2} / \mathrm{g}, 280,000 \mathrm{~cm}^{2} / \mathrm{g}$. Using the value for devitrified tuff, the specific surface area is $\sim 35$ times larger than the reported gas adsorption surface area of the Ottawa sand $\left(\sim 1000 \mathrm{~cm}^{2} / \mathrm{g}\right)$ of Leecaster et al. (2012). We use the gas adsorption surface area of the sand here because we only have a gas adsorption surface area for the tuff, and it is best to use the same measurement method when calculating surface area ratios. If we assume that the Soda Lake and Yucca Mountain tuffs have similar specific surface areas, the fine-scale surface area available for sorption in a fracture at Soda Lake would be about 35 times greater than the fine-scale area available for sorption on Ottawa sand. This difference in fine-scale surface area would have the same effect as increasing the $K_{a}$ value of the tuff by a factor of 35 in Equation (6.1), resulting in a fracture half-aperture estimate 35 times greater than the one obtained assuming the $K_{a}$ value for Ottawa sand. The resulting estimate would be $3.1 \mathrm{e}-3 \mathrm{~cm}$, or $31 \mu \mathrm{m}$, which still seems low for supporting the high flow rates at Soda Lake.

This adjusted estimate is probably low because of the implicit assumption in Equation (6.1) that the fracture approximates a parallel-plate channel at macroscopic scales. In reality, surface roughness and rugosity will increase the surface area significantly over that of a parallel-plate channel (e.g., Figure 6.6). However, it is virtually impossible to estimate how much surface area the roughness might add without at least examining some representative fracture surfaces, and even a few isolated samples would not necessarily be representative of the entire fracture system accessed in the field tracer test.

\section{Heat Transfer Surface Area}

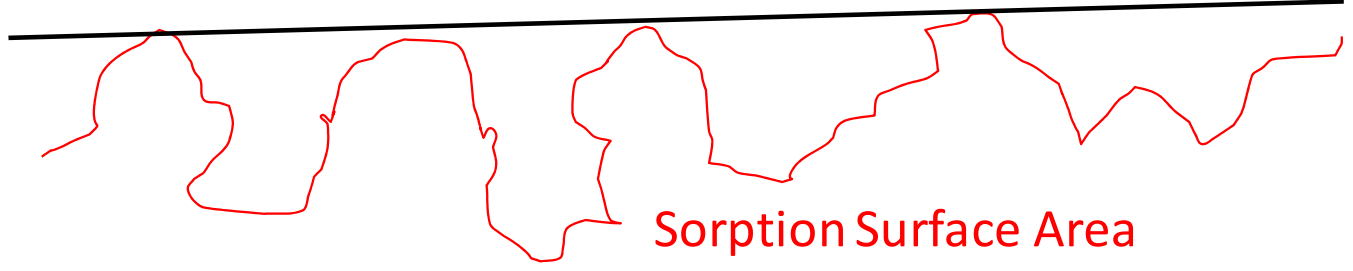

Figure 6.6. Illustration of difference between sorption surface area and heat transfer surface area at a fracture surface.

\subsubsection{Alternative Dual-Porosity Model: The Rim-Zone Model}

To avoid making an assumption that would effectively dictate the surface area estimate, an alternative conceptual model of the flow/transport system was considered that does not directly rely on a surface roughness estimate. In this conceptualization, a dual-porosity system with limited diffusion into the matrix but no sorption in fractures was assumed (Figure 6.7). The limited matrix volume can be assigned properties that are consistent with a porous medium, which allows for a more defensible tie to the porous media experiments of Leecaster et al. (2012) and also for a more defensible translation of the Ottawa sand porous medium to a volcanic tuff porous medium using some of the arguments presented above. The rationale for this conceptualization is that real fractures tend to have rough walls, damage zones, and fracture coating minerals that tend to enhance the effective porosity for a short distance into an otherwise very low-porosity matrix. This enhanced diffusion zone, or rim zone, has been recognized by others who have studied tracer or contaminant transport in fractured rock systems (Cvetkovic 2010; Andersson et al. 2002; Winberg et al. 2002). The rim-zone model can behave as a single-porosity model if the rim zone is thin relative to the distance that tracers can diffuse through the zone over the time scale of a tracer test. In 
this case, the rim zone will act the same a highly sorptive fracture surface, with the advantage that the actual surface roughness need not be specified because the sorptive surface area is determined by the properties of the rim zone (namely, the porosity and the specific surface area of the rim-zone material). Because a single-porosity model fit the Saf-T breakthrough curve slightly better than either of the dualporosity models with a semi-infinite matrix, the rim-zone model seemed like a very sensible approach to take.

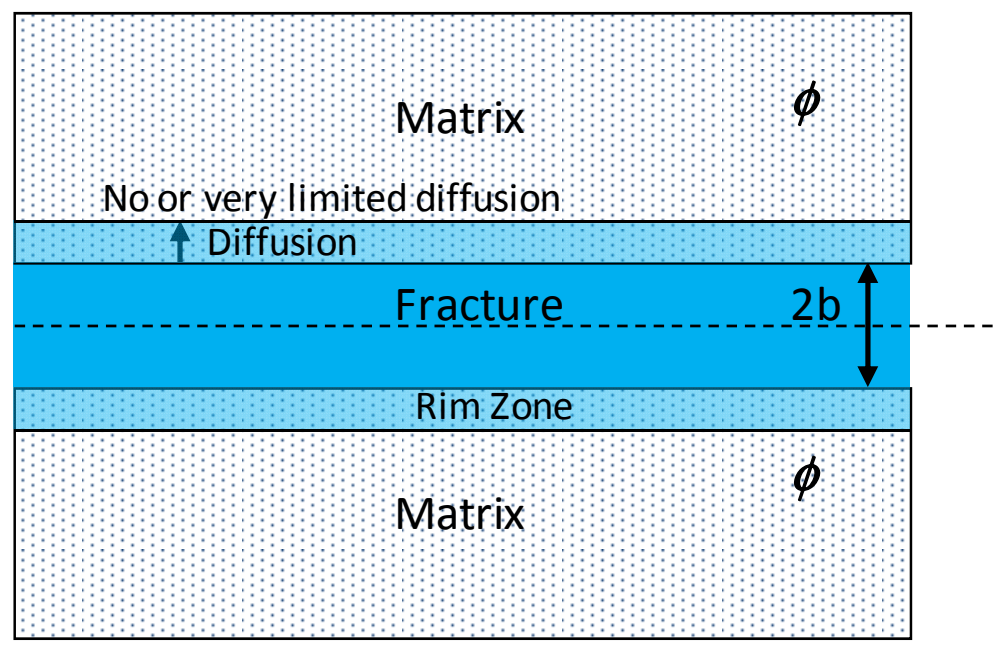

Figure 6.7. Schematic of the rim-zone model.

To use the rim-zone model (as it will henceforth be referred), it is necessary to estimate the thickness of the rim zone relative to the open fracture half-aperture. It is also necessary to use realistic estimates of tracer diffusion coefficients at reservoir temperatures because access to the rim zone is diffusion controlled, and because it was concluded that fracture retardation is far more important than matrix retardation, the rim-zone thickness should logically be limited to distance scales that tracers can readily diffuse across over test time scales, which is a function of the diffusion coefficient. This limitation will ensure that the rim zone acts as a sorptive fracture surface rather than a sorptive matrix (consistent with the test interpretations).

The effective porosity of the rim zone is an uncertainty, and it is not easily measured or estimated without core samples that contain natural fractures, so rather than assume a fixed value, a range from 0.1 to 0.4 was used to cover a reasonable range of possibilities (specifically, values of $0.1,0.15,0.2,0.3$, and 0.4 were used). The diffusion coefficient of the tracers in the rim zone was estimated using the following logic. It is reasonably well documented that fluorinated benzoates have free-water diffusion coefficients of $6 \mathrm{e}-6$ to $7.5 \mathrm{e}-6 \mathrm{~cm}^{2} / \mathrm{s}$ at $25^{\circ} \mathrm{C}$ (Bowman 1984; Farnham et al. 1997). Although diffusion coefficients for 1,6-NDS and Saf-T are not known, given that they are both larger molecules than fluorinated benzoates, with at least one additional benzene ring in their molecular structure, their free-water diffusion coefficients should be somewhat smaller at the same temperature. For the purposes of this study, we assumed a value of $5 \mathrm{e}-6 \mathrm{~cm}^{2} / \mathrm{s}$ at $25^{\circ} \mathrm{C}$. This value must be corrected for the elevated temperature at Soda Lake. The temperature dependence of diffusion coefficients is given by

$$
\frac{D_{2}}{D_{1}}=\frac{T_{2}}{T_{1}} \frac{\mu_{1}}{\mu_{2}}
$$


where $D_{x}$ is the diffusion coefficient at temperature $\mathrm{x}\left(\mathrm{cm}^{2} / \mathrm{s}\right), T_{x}$ is temperature $\mathrm{x}\left({ }^{0} \mathrm{~K}\right)$, and $\mu_{x}$ is viscosity at temperature $\mathrm{x}(\mathrm{g} / \mathrm{cm}-\mathrm{s})$.

For the temperature dependence of water viscosity at moderate pressures, we used the following equation, which is reported to be accurate to $2.5 \%$ from $0^{\circ} \mathrm{C}$ to $370^{\circ} \mathrm{C}$ (Likhachev 2003):

$$
\mu(T)=2.414 \times 10^{-4} 10^{\frac{247.8}{(T-140)}}
$$

where $\mu$ is viscosity (g/cm-s) and $T$ is temperature $\left({ }^{\circ} \mathrm{K}\right)$.

The free-water diffusion coefficient must be further corrected to account for the tortuosity in the rim zone. For this we used the following expression developed by Reimus et al. (2007) based on experimentally measured diffusion coefficients in volcanic rock matrices of known porosity from the Nevada Test Site:

$$
\log \left(\frac{D_{m}}{D^{*}}\right)=3.0681 \varnothing-1.979
$$

where $D_{m}$ is the matrix diffusion coefficient $\left(\mathrm{cm}^{2} / \mathrm{s}\right), D^{*}$ is the free-water diffusion coefficient $\left(\mathrm{cm}^{2} / \mathrm{s}\right)$, and $\phi$ is the porosity of the matrix or rim zone.

Another uncertainty in the rim zone is the effective retardation factor for a reactive tracer. To address this uncertainty, we assume that Saf-T has the same $K_{a}$ value on the Soda Lake volcanic tuff as on the Ottawa sand in the $160^{\circ} \mathrm{C}$ experiments of Leecaster et al. (2012). We further assume that the specific surface area of the Soda Lake tuff is approximately the same as the volcanic tuffs at Yucca Mountain; i.e., $\sim 35,000 \mathrm{~cm}^{2} / \mathrm{g}$. However, to allow for some uncertainty, we consider cases where the tuff-specific surface area could be as low as $15,000 \mathrm{~cm}^{2} / \mathrm{g}$ and as high as $100,000 \mathrm{~cm}^{2} / \mathrm{g}$ (within the range observed for Yucca Mountain tuffs). We intuitively expect that it is more likely that the effective specific surface area will be larger than $35,000 \mathrm{~cm}^{2} / \mathrm{g}$ rather than smaller because the tuffs are likely to contain minerals that are more sorptive per unit surface area than the quartz sand, which would result in a larger effective specific surface area. We also assume a specific surface area of $950 \mathrm{~cm}^{2} / \mathrm{g}$ for the Ottawa sand used by Leecaster et al. (2012), which is the average of gas adsorption measurements on Ottawa sand reported by others (Kibbey et al. 2007; Reimus et al. 2010). Assuming this specific surface area for the Ottawa sand, we calculate a $K_{a}$ value of $2.4 \mathrm{e}-4 \mathrm{~cm}^{3} / \mathrm{cm}^{2}$ for Saf-T using Equation (6.1) and the experimental results of Leecaster et al. (2012).

Given these assumptions, the effective retardation factor in the rim zone can be calculated as a function of the rim-zone porosity, $\phi$, as follows:

$$
R_{\text {rim }}=1+\frac{\rho_{S}(1-\varnothing) S S A}{\emptyset} K_{a}
$$

where $\rho_{s}$ is the density of the tuff, $\mathrm{g} / \mathrm{cm}^{3}\left[\rho_{s}(1-\phi)=\right.$ bulk density of tuff, $\left.\mathrm{g} / \mathrm{cm}^{3}\right]$, and SSA is the specific surface area of the tuff, $\mathrm{cm}^{2} / \mathrm{g}$. Note that $K_{d}=\operatorname{SSA}\left(K_{a}\right)$. 
The effective fracture retardation factor is the volume-weighted sum of the retardation factor in the open fracture space (i.e., 1) and the retardation factor in the rim zone (i.e., $R_{\text {rim }}$ ). Mathematically, this is expressed as

$$
R_{f}=\frac{b+x \emptyset R_{\text {rim }}}{b+x \emptyset}
$$

where $x$ is the thickness of the rim zone $(\mathrm{cm})$.

Upon rearrangement, Equation (6.6) becomes

$$
\frac{x}{b}=1-\frac{\left(R_{f}-R_{\text {rim }}\right)}{\left(1-R_{\text {rim }}\right)} / \frac{\left(R_{f}-R_{\text {rim }}\right)}{\left(1-R_{\text {rim }}\right)} \emptyset
$$

Thus, for any assumed/calculated values of $\phi$ and $R_{\text {rim }}$, and for an observed value of $R_{f}$ (i.e., $\sim 3.6$ from the field tracer test) the thickness of the rim zone as a fraction of the fracture half-aperture is fixed. Equation (6.7) implies that the fracture half-aperture and rim-zone thickness can take on any values provided their ratio satisfies this equation. However, the rim-zone thickness has a practical upper limit that is determined by the ability of tracers to diffuse across it during the time scale of a tracer test. Once the rim-zone thickness exceeds this upper limit, the tracer breakthrough curve will begin to deviate from the breakthrough curve predicted by a model that assumes retardation only in the fractures. Given that the ratio of rim-zone thickness to fracture half-aperture thickness is fixed by Equation (6.7), an upper limit for the rim-zone thickness implies an upper limit for the fracture half-aperture. Thus, for a given value of $x / b$, we can use the RELAP model to determine an upper bound for the fracture half-aperture by systematically increasing the values of $x$ and $b$ (while keeping their ratio constant) until the predicted Saf-T breakthrough curve begins to deviate significantly from the observed breakthrough curve. Note that there is no practical way of determining a lower bound for $x$ or $b$; even unrealistically low values of these parameters will yield good fits to the Saf-T breakthrough curve provided their ratio satisfies Equation (6.7).

\subsubsection{Half-Aperture Estimates from Rim-Zone Model}

Table 6.5, Table 6.6, and Table 6.7 list upper-bound estimates of fracture half-apertures $\left(b_{\max }\right)$ as a function of assumed rim-zone porosity for different assumed values of the specific surface area of the Soda Lake rim-zone tuff $\left(35,000,100,000\right.$, and $15,000 \mathrm{~cm}^{2} / \mathrm{g}$, respectively). For each porosity in Table 6.5, Table 6.6, and Table 6.7, a rim-zone retardation factor and $x / b$ value, calculated using Equations (6.5) and (6.7), respectively, are listed along with the fracture half-aperture at which fits to the Saf-T data start to deviate significantly from the observed data. Figure 6.8 shows an example of what is considered a significant deviation. Prior to fitting the Saf-T breakthrough curve, the mean residence time, Peclet number, and tracer mass fraction participating in the test were estimated for each rim-zone porosity and $x / b$ value by fitting the $1,6-$ NDS breakthrough curve assuming no sorption of the $1,6-\mathrm{NDS}$. The resulting best estimates of these parameters, along with the estimated matrix diffusion coefficient at $180^{\circ} \mathrm{C}$ (calculated via Equations (6.2)-(6.4)), are listed in Table 6.5, Table 6.6, and Table 6.7. In all cases, the Peclet number and mass fraction remained essentially fixed at 4 and 0.27 , respectively, and the mean residence time varied in proportion to $b /(x \phi+b)$. The resulting parameter values were then used in 
the Saf-T simulations with the $x$ and $b$ values systematically increased but $x / b$ remained constant. The rim-zone retardation factor was used as a fitting parameter in the RELAP simulations, but this was only to verify that the RELAP model returned the value calculated in Equation (6.5), which was always the case (at least until the model began to deviate significantly from the data).

Table 6.5. Rim-zone model parameters, including upper-bound estimates of fracture half-apertures $\left(b_{\max }\right)$, yielding good fits to the Saf-T breakthrough curve assuming a tuff-specific surface area of $35,000 \mathrm{~cm}^{2} / \mathrm{g}$.

\begin{tabular}{cccccccc}
\hline$\phi_{\text {rim }}$ & $D_{m}, \mathrm{~cm}^{2} / \mathrm{s}$ & $\tau, \mathrm{hr}$ & Pe & mass frac & $R_{\text {rim }}$ & $x / b$ & $b_{\max }, \mathrm{cm}$ \\
\hline 0.1 & $1 \mathrm{e}-6$ & 229 & 3.9 & 0.2725 & 21.4 & 1.46 & 0.024 \\
0.15 & $1.4 \mathrm{e}-6$ & 206 & 4 & 0.2675 & 13.9 & 1.69 & 0.03 \\
0.2 & $2 \mathrm{e}-6$ & 184 & 4 & 0.2675 & 10.1 & 2.01 & 0.0375 \\
0.3 & $4 \mathrm{e}-6$ & 132 & 4 & 0.27 & 6.30 & 3.21 & 0.0375 \\
0.4 & $8 \mathrm{e}-6$ & 61 & 4 & 0.2675 & 4.41 & 8.07 & 0.024 \\
\hline
\end{tabular}

Table 6.6. Rim-zone model parameters, including upper-bound estimates of fracture half-apertures $\left(b_{\max }\right)$, yielding good fits to the Saf-T breakthrough curve assuming a tuff-specific surface area of $100,000 \mathrm{~cm}^{2} / \mathrm{g}$.

\begin{tabular}{cccccccc}
\hline$\phi_{\text {rim }}$ & $D_{m}, \mathrm{~cm}^{2} / \mathrm{s}$ & $\tau, \mathrm{hr}$ & $P e$ & mass frac & $R_{\text {rim }}$ & $x / b$ & $b_{\max }, \mathrm{cm}$ \\
\hline 0.1 & $1 \mathrm{e}-6$ & 251 & 3.9 & 0.2725 & 59.4 & 0.47 & 0.045 \\
0.15 & $1.4 \mathrm{e}-6$ & 244 & 3.9 & 0.2725 & 37.8 & 0.51 & 0.063 \\
0.2 & $2 \mathrm{e}-6$ & 236 & 3.9 & 0.2725 & 26.9 & 0.56 & 0.0825 \\
0.3 & $4 \mathrm{e}-6$ & 218 & 3.9 & 0.2725 & 16.1 & 0.69 & 0.1125 \\
0.4 & $8 \mathrm{e}-6$ & 189 & 4 & 0.2675 & 10.7 & 0.91 & 0.2 \\
\hline
\end{tabular}

Table 6.7. Rim-zone model parameters, including upper-bound estimates of fracture half-apertures $\left(b_{\max }\right)$, yielding good fits to the Saf-T breakthrough curve assuming a tuff-specific surface area of $15,000 \mathrm{~cm}^{2} / \mathrm{g}$.

\begin{tabular}{cccccccc}
\hline$\phi_{\text {rim }}$ & $D_{m}, \mathrm{~cm}^{2} / \mathrm{s}$ & $\tau, \mathrm{hr}$ & Pe & mass frac & $R_{\text {rim }}$ & $x / b$ & $b_{\max }, \mathrm{cm}$ \\
\hline 0.1 & $1 \mathrm{e}-6$ & 182 & 4 & 0.27 & 9.76 & 4.22 & 0.0135 \\
0.15 & $1.4 \mathrm{e}-6$ & 137 & 4 & 0.27 & 6.51 & 5.94 & 0.0135 \\
0.2 & $2 \mathrm{e}-6$ & 86 & 4 & 0.27 & 4.89 & 10.06 & 0.0105 \\
0.3 & $4 \mathrm{e}-6$ & NA & NA & NA & $3.27^{(\mathrm{a})}$ & NA & NA \\
0.4 & $8 \mathrm{e}-6$ & NA & NA & NA & $2.46^{(\mathrm{a})}$ & NA & NA \\
\hline
\end{tabular}

(a) Calculated rim-zone retardation factor is less than the observed fracture retardation factor, so the rim-zone model cannot be applied (an infinite thickness will still not provide enough retardation).

Although the fracture half-aperture estimates in Table 6.5, Table 6.6, and Table 6.7 are upper bounds, they are not unreasonable estimates of actual fracture half-apertures in the Soda Lake flow system. The half-apertures in Table 6.5 are considered to be the best estimates because they were derived from what are considered to be the most probable value of the tuff-specific surface area. The estimates of Table 6.6 
are considered more likely than those of Table 6.7 because it is considered more likely that alteration minerals forming on fracture surfaces will have higher, rather than lower, specific surface areas than the unaltered volcanic tuff.

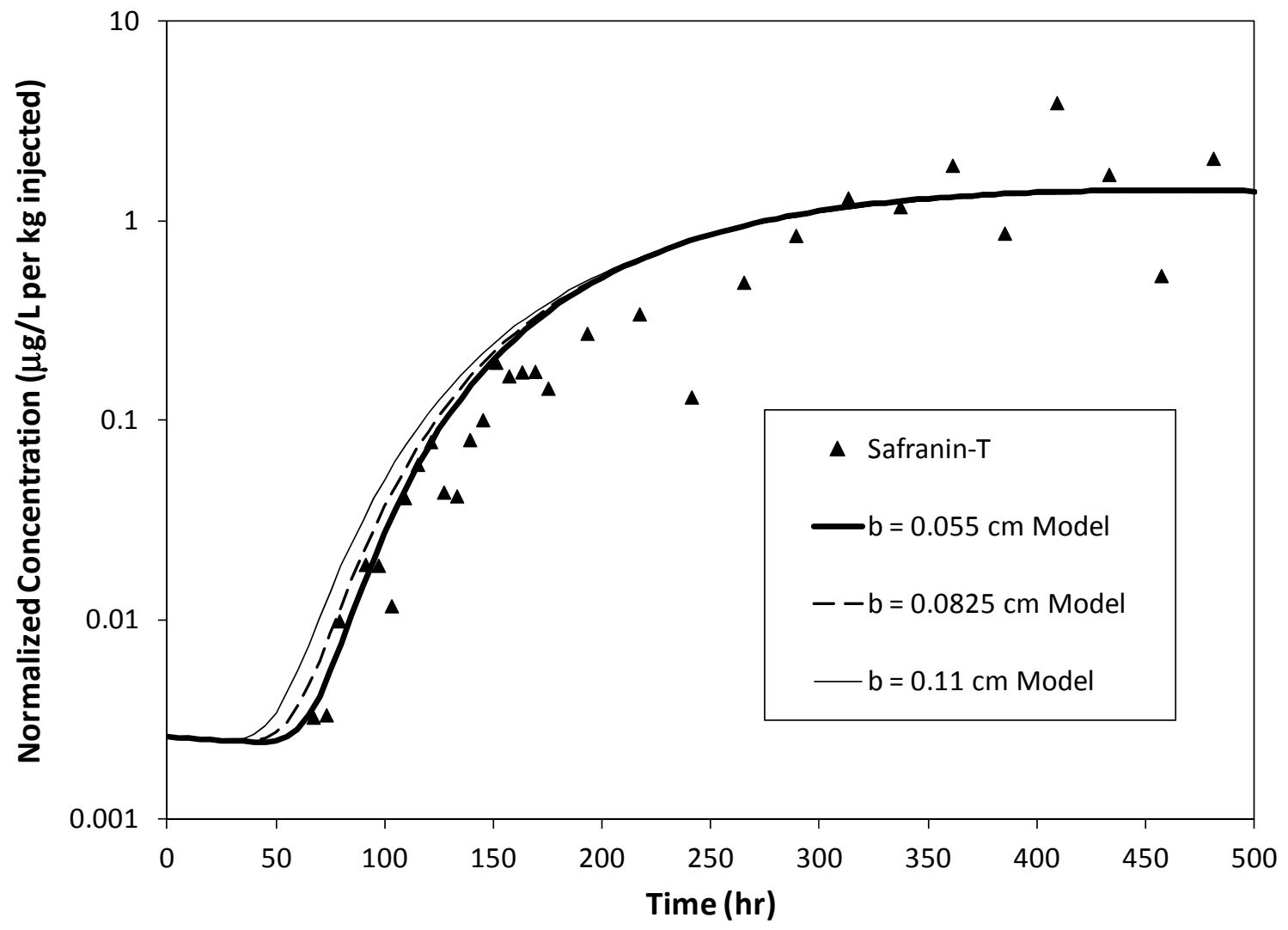

Figure 6.8. Rim-zone model fits to Saf-T breakthrough curve using different values of the fracture halfaperture $b$ (with $x / b$ fixed). The thin solid line is an example of what was considered a significant deviation between the model and the data, and the dashed line was considered a minimally acceptable fit (thus $b_{\max }$ was taken to be $0.0825 \mathrm{~cm}$ ). Conditions are $\phi_{\text {rim }}=0.2$ and tuff-specific surface area $=100,000 \mathrm{~cm}^{2} / \mathrm{g}$ (third row of Table 6.6).

\subsubsection{Lower-Bound Half-Aperture Estimates Based on Flow Calculations}

Fracture half-apertures significantly smaller than those listed in Table 6.5 and Table 6.7, and especially Table 6.7, would restrict flow too much to support the high flow rates used at Soda Lake. To estimate half-apertures that are consistent with the Soda Lake flows, we assume that the cubic law of flow through parallel-plate fractures applies, as follows:

$$
Q=-\frac{2 W \rho g b^{3}}{3 \mu} \frac{\Delta h}{L}
$$

where $\quad Q=$ volumetric flow rate, $\mathrm{m}^{3} / \mathrm{s}$

$W=$ fracture width, $\mathrm{m}$

$\rho=$ density of water, $\mathrm{kg} / \mathrm{m}^{3}$

$g=$ gravity acceleration, $9.8 \mathrm{~m} / \mathrm{s}^{2}$ 


$$
\begin{aligned}
b & =\text { fracture half-aperture, } \mathrm{m} \\
\mu & =\text { viscosity of water, } \mathrm{kg} / \mathrm{m}-\mathrm{s} \\
\Delta h & =\text { head difference between exit and entrance to fracture, } \mathrm{m} \\
L & =\text { length of fracture, } \mathrm{m} .
\end{aligned}
$$

Note that the fracture half-aperture calculated from Equation (6.8) will always be smaller than the transport half-aperture determined from tracer test interpretations because hydraulic apertures tend to be biased low by fracture "pinch points" that restrict flow, whereas tracer transport apertures better reflect true volumetric/geometric apertures (Tsang 1992). The latter apertures will dictate heat transfer in a geothermal system. Hydraulic and transport apertures are equal only for parallel-plate channels, and in all other cases hydraulic apertures are smaller than transport apertures. Thus, a reasonable estimate of the hydraulic aperture can serve as a good lower-bound estimate of the geometric aperture in a flow system.

For a rectangular fracture, geometry dictates that

$$
\mathrm{V}=2 \mathrm{bWL}
$$

where $V$ is the fracture or flow system volume $\left(\mathrm{m}^{3}\right)$.

Equations (6.8) and (6.9) can be solved for $b$ to obtain the following:

$$
b=\left(-\frac{3 Q \mu}{2 W \rho g} \frac{L}{\Delta h}\right)^{1 / 3}=\frac{V}{2 W L}
$$

Equation (6.10), in turn, can be solved for $W$ to yield the following:

$$
W=\left[\left(\frac{V}{2 L}\right)\left(-\frac{2 \rho g}{3 Q \mu} \frac{\Delta h}{L}\right)^{1 / 3}\right]^{3 / 2}
$$

If this value for $\mathrm{W}$ is inserted back into either Equation (6.8) or (6.9), a hydraulic aperture estimate is obtained. Note that a large value of $W$ does not necessarily imply an extremely wide fracture, but rather it implies numerous fractures of moderate width.

Equations (6.8) through (6.11) are valid for linear flow through a parallel-plate fracture. An assumption of radial flow may be more applicable for the Soda Lake tracer test, particularly near the injection and production wells. For radial flow, Equations (6.8) and (6.9) become

$$
Q=-\frac{N 4 \pi \rho g b^{3}}{3 \mu \ln \left(\frac{r_{L}}{r_{w}}\right)} \frac{\Delta h}{2}
$$

and

$$
\mathrm{V} / 2=\pi 2 \mathrm{br}_{\mathrm{L}}^{2} \mathrm{~N}
$$

where $r_{L}$ is approximately half the distance between the injection and production wells $(\mathrm{m}), r_{w}$ is the well radius $(\mathrm{m})$, and $N$ is the number of radial fractures. 
Equations (6.12) and (6.13) are written for half the distance between the injection and production wells (half the head difference in Equation (6.12), half the system volume in Equation (6.13), and half the distance between the wells, $r_{L}$, in both equations). The rationale is that radial sweeps to/from the injection and production wells only need to reach approximately half the distance between the wells to provide good hydraulic connection between the wells.

Equations (6.12) and (6.13) can be solved for $b$, and in turn, the resulting expressions can be set equal to each other and solved for $N$ to yield the following:

$$
N=\left[\left(\frac{V}{04 \pi r_{L}^{2}}\right)\left(-\frac{4 \pi \rho g \Delta h}{6 Q \mu \ln \left(\frac{r_{\mathrm{L}}}{\mathrm{r}_{\mathrm{W}}}\right)}\right)^{1 / 3}\right]^{3 / 2}
$$

Inserting this result back into either Equation (6.12) or (6.13) yields a radial-flow hydraulic aperture estimate.

For the Soda Lake tracer test, the volume swept by the tracers in the test can be estimated from

$$
\mathrm{V}=\mathrm{Q} \tau
$$

where $V$ is the system volume $\left(\mathrm{m}^{3}\right), Q$ is the volumetric injection flow rate $\left(\mathrm{m}^{3} / \mathrm{hr}\right)$, and $\tau$ is the mean conservative tracer residence time (hr).

Using a mean residence time of $\sim 250 \mathrm{hr}$ for the 1,6-NDS, and a flow rate $Q$ equal to $30 \%$ of $800 \mathrm{gpm}$ (i.e., $30 \%$ of the injection flow rate at Soda Lake, because only about $30 \%$ of the tracer mass was recovered), Equation (6.9) yields a volume of $\sim 13,600 \mathrm{~m}^{3}$ that was interrogated by the tracers.

Besides $V$ and $Q$, the other system-specific parameters needed in the preceding equations to estimate hydraulic apertures are $\Delta h$, the head difference between the wells; $L$ (or $r_{L}$ ), the distance between the wells; and $r_{w}$, the well radius. For the Soda Lake tracer test, $L$ is $\sim 550 \mathrm{~m}$, and $r_{w}$ is $\sim 0.3 \mathrm{~m}(12-\mathrm{in}$.diameter wells in open sections). The head difference $\Delta h$ is estimated to be approximately $525 \mathrm{~m}$ under the operating conditions of the tracer test (personal communication with D. Benoit, Magma Energy Corp.). We actually use a value of $L=750 \mathrm{~m}$ in the case of a linear flow system to account for the possibility that fractures transmitting flow extend beyond the interwell separation distance and initially transmit some of the injected tracer mass in the direction opposite of the most direct path to the production well, and also to account for the fact that the vertical travel distance may be as much as $500 \mathrm{~m}$ between the injection and production wells due to their different completion depths and open interval thicknesses. Also, $r_{L}$ was assumed to be $325 \mathrm{~m}$ instead of $550 / 2=275 \mathrm{~m}$ to ensure good overlap of the radial "disks" between the wells (i.e., $\sim 100$-m overlap) and also to allow for some of the vertical travel distance considerations. Note that the radial disks implicitly account for significant fracture extension beyond the separation distance between the two wells.

Using all the known and assumed parameter values and the equations discussed above, we calculate hydraulic half-apertures of $\sim 0.0019 \mathrm{~cm}$ for linear flow and $\sim 0.0031 \mathrm{~cm}$ for radial flow. These can be considered absolute lower-bound estimates of fracture half-apertures for the Soda Lake system because they are calculated assuming smooth parallel-plate flow channels. Any surface roughness or rugosity will result in significantly larger estimates. The estimate for radial flow is larger than that for linear flow 
because a large fraction of the pressure drop in radial flow occurs near the injection and production wells, whereas in linear flow a linear pressure gradient is assumed between the wells. The convergence or divergence of flow near the wells in radial flow forces the fracture half-aperture to be larger to satisfy the overall pressure drop constraint. The radial flow assumption is probably more realistic than the linear flow assumption, although linear flow can be shown to be a good assumption close to wells when flow is highly channelized.

\subsubsection{Conclusions from Semi-Analytical Model Analysis}

The fracture half-aperture estimates in Table 6.5, which are equivalent to fracture SA/V ratios, are considered to be the best estimates from this analysis. However, the estimates listed in Table 6.6 and Table 6.7 are also considered plausible given the potential range of specific surface areas assumed for the tuff minerals in the rim zone of Soda Lake fractures. The estimates of Table 6.6 are considered to be more probable than those of Table 6.7, because it is considered more likely that alteration minerals forming on fracture surfaces will have higher specific surface areas than those assumed for Table 6.5.

Although the values listed in Table 6.5, Table 6.6, and Table 6.7 are technically upper bounds that are 1 to 2 orders of magnitude larger than the lower-bound estimates determined from flow resistance considerations (i.e., 0.0025 to $0.004 \mathrm{~cm}$ ), we consider the upper bounds to be better estimates of true geometric half-apertures than the lower bounds because the lower bounds can be biased very low by a few (in principle, just one) significant flow restrictions in an otherwise high-aperture fracture.

Taken together, Table 6.5 , Table 6.6 , and Table 6.7 provide a relatively wide range of upper bounds for fracture half-apertures as a function of rim-zone porosity and specific surface area of the minerals in the rim zone. A key assumption in developing these estimates is that the surface-area-based Saf-T partition coefficient ( $K_{a}$ value) is the same on the rim-zone minerals as on the Ottawa sand in the experiments of Leecaster et al. (2012). This assumption results in a mass-based Saf-T partition coefficient ( $K_{d}$ value) that ranges from 15 to 100 times greater than the experimentally determined $K_{d}$ value of the Ottawa sand, depending on the specific surface area assumed for the rim-zone minerals.

Estimates of the heat transfer surface area are best expressed on a per unit volume basis, equal to the reciprocal of the fracture half-aperture estimates of Table 6.5, Table 6.6, and Table 6.7. For the volume interrogated in the Soda Lake tracer test, i.e., $13,500 \mathrm{~m}^{3}$ (as determined from the 1,6-NDS mean residence time multiplied by $30 \%$ of the injection flow rate), and assuming a fracture half-aperture of $0.04 \mathrm{~cm}$ $(0.0004 \mathrm{~m})$, the total surface area interrogated is calculated to be $13,500 / 0.0004=3.375 \times 10^{7} \mathrm{~m}^{2}$.

\subsubsection{Recommendations for Improving Surface Area Estimates}

The SA/V ratio estimates from the analysis presented here are quite uncertain because of the many assumptions required to do the calculations. This uncertainty could be reduced if additional site-specific information were available.

A significant reduction in uncertainty could be achieved by conducting Saf-T sorption measurements with site-specific minerals or a mineral assemblage that approximates the minerals present at Soda Lake. Ideally, the mineral assemblage would match what is present in fracture flow pathways, especially if it is notably different than the bulk rock matrix mineralogy. Column flow experiments similar to the ones conducted with the Ottawa sand by Leecaster et al. (2012) are suggested for doing the sorption 
measurements, with the columns well characterized as to their porosity and pore volume. The goal would be to obtain an estimate of the site-specific mass-based partition coefficient ( $K_{d}$ value) of Saf-T for direct use in Equation (6.5) in place of $S S A\left(K_{a}\right)$. These tests would also help verify whether the presence of the mineral surfaces has any influence on the thermal degradation of the Saf-T (the previous thermal degradation experiments of Rose et al. [2012] were conducted without any rock present). However, it should be noted that the interpretation of the tracer breakthrough curves to obtain surface area estimates were ultimately not highly dependent on the Saf-T thermal degradation rate because the surface area estimate was driven mainly by the first arrival time and early shape of the Saf-T breakthrough curve, which was quite insensitive to the assumed degradation rate.

Any independent information about the rim-zone characteristics at Soda Lake would be extremely useful for reducing uncertainty in surface area estimates from the rim-zone model. Estimates of rim-zone thickness, porosity, and mineralogy, as well as any gradients in porosity and mineralogy across the rim zone, would be especially useful. Naturally fractured core would probably be the best source material for obtaining this information, although it is recognized that the relatively small samples derived from core are unlikely to represent the full range of characteristics present in the field fracture system. The porosity of the rim zone is as important in determining the rim-zone retardation factor as the Saf-T partition coefficient (see Equation (6.5)), and the rim-zone thickness is critical for constraining the estimate of fracture half-aperture via Equation (6.7). These estimates would probably have to be obtained by careful microscopic examination of fracture surfaces. The best way to do this might be to examine multiple thin sections cut perpendicular to fracture surfaces to achieve good measurement statistics, although nondestructive methods (e.g., x-ray microtomography of fracture surfaces) might be preferable if they are not prohibitively expensive.

Gas adsorption surface area measurements on the tuff material used in the column tests (as well as on the sand used in the previous experiments) would be helpful to establish whether the surface area-based partition coefficients on the sand and tuff minerals are similar, as was assumed in the analysis here. If the surface area-based partition coefficients are similar, it would suggest that Saf-T partition coefficients can be estimated reasonably well for other geothermal systems from surface area measurements alone. However, if the surface area-based partition coefficients are quite different, it would suggest that there is an important mineralogic influence of Saf-T sorption behavior that would require more detailed sitespecific sorption characterization in any system in which Saf-T is used as a sorbing tracer.

Measurements of naphthalene sulfonate and Saf-T diffusion coefficients in the rim zone and in the bulk rock matrix would be useful, although these could be difficult and relatively expensive, and the reduction in uncertainty would be less than with the other measurements mentioned above. The sensitivity of surface area estimates to tracer diffusion coefficients is significantly less than the sensitivity to the rim-zone partition coefficient, porosity, and thickness.

\subsubsection{Numerical Model (TOUGHREACT V1.2)}

A numerical model was developed using the TOUGHREACT code (Xu et al. 2006) to simulate the Soda Lake tracer test and to investigate the efficacy of using single-well injection/withdrawal tests at the site for reservoir characterization. Site characterization and tracer parameters from fitting the two-well tracer test data are used for the single-well injection/withdrawal test models. The TOUGHREACT code 
was selected to take advantage of the MINC (Pruess 1983) option for simulating a fractured reservoir and options for simulating sorbing and thermally decay of tracers.

The initial model has a two-dimensional plan view grid (x,y) with a constant thickness (z) of $300 \mathrm{~m}$. The grid spacing in the $\mathrm{x}, \mathrm{y}$ is $10 \mathrm{~m}$ between and near the injection and production wells and then increases to a final $1-\mathrm{km}$ spacing near the edges (overall domain size is $5.47 \mathrm{~km} \times 2.59 \mathrm{~km}$ ). The wells are spaced $550 \mathrm{~m}$ apart. We used $1 / 2$ symmetry for the problem with the corresponding reductions in injection and withdrawal rates (full values were $800 \mathrm{gpm}$ for the injection well and $885 \mathrm{gpm}$ for the production well) and tracer mass. Boundary conditions are no-flow along the top, bottom, and line of symmetry (e.g., $\mathrm{y}=0$ ) with dirichlet conditions specified along the other boundaries. The MINC option in TOUGHREACT is used for simulating the fractured reservoir. In these simulations, the domain is divided into five continua (one for the fracture network and four for the matrix).

Thermal decay of Saf-T was directly simulated in the model based on parameters measured in the laboratory (Rose et al. 2012). This approach is in contrast to the RELAP modeling approach, where heat transfer was not included in the model and thermal decay was corrected for in advance of the model interpretations. Initial sorption properties of Saf-T (partition coefficients) are based on fitting the tracer test data from the Soda Lake two-well tracer test as discussed below. Results from ongoing laboratory studies of Saf-T transport with site materials could be incorporated in this modeling effort to help constrain and guide these modeling fitting estimates.

Initial reservoir temperatures were set to $180^{\circ} \mathrm{C}$ and the temperature of the injection fluid was set at $71^{\circ} \mathrm{C}$. Injection and withdrawal started at time $=0$ and ran for $28 \mathrm{~d}(800 \mathrm{gpm}$ for the injection well and 885 gpm for the production well, scaled to the model symmetry). For simplicity in this initial model, the tracers were injected during the first hour of the test followed by freshwater injection for the duration of the simulation period ( $50 \mathrm{~kg}$ of 1,6-NDS and $90 \mathrm{~kg}$ of Saf-T, scaled to the model symmetry). During the field test the 1,6-NDS was injected in about $0.5 \mathrm{hr}$ at the start of the test and the Safranin-T was injected afterward over a period of about $3 \mathrm{hr}$ due to injection difficulties. In addition, for these initial simulations, re-injection of the partial mass recovered from the production well was not included.

A manual calibration process was used for this initial model. Parameters were varied based on visual comparison of simulated results with the tracer concentration measurements from the production well. The parameters varied during this fitting process included fracture and matrix volumes, matrix porosity, matrix diffusion coefficient, and Saf-T partition coefficients (different values for fractures and matrix). An initial fracture spacing of $10 \mathrm{~m}$ was used but was not varied. The impact of this parameter could be investigated in further sensitivity studies.

Figure 6.9 shows a comparison of the simulated 1,6-NDS and Saf-T concentrations at the production well with the measured values. Maximum simulated Saf-T concentrations were approximately two times the measured values during the peak for this case. Simulated 1,6-NDS and Saf-T plumes within the fractures at $4 \mathrm{~d}$ are also plotted in Figure 6.9. Parameters for this model are shown in Figure 6.8.

Although it is difficult to make a direct visual comparison of the RELAP model results shown in Figure 6.8 and the TOUGHREACT results shown in Figure 6.9 (because of the large differences in uncorrected and corrected Saf-T concentrations), the results are nonetheless in good agreement. The agreement between the Saf-T simulations is most readily apparent in the over-prediction of the concentrations from about 7 to $12 \mathrm{~d}$ and the significant under-prediction of the last two data points. 


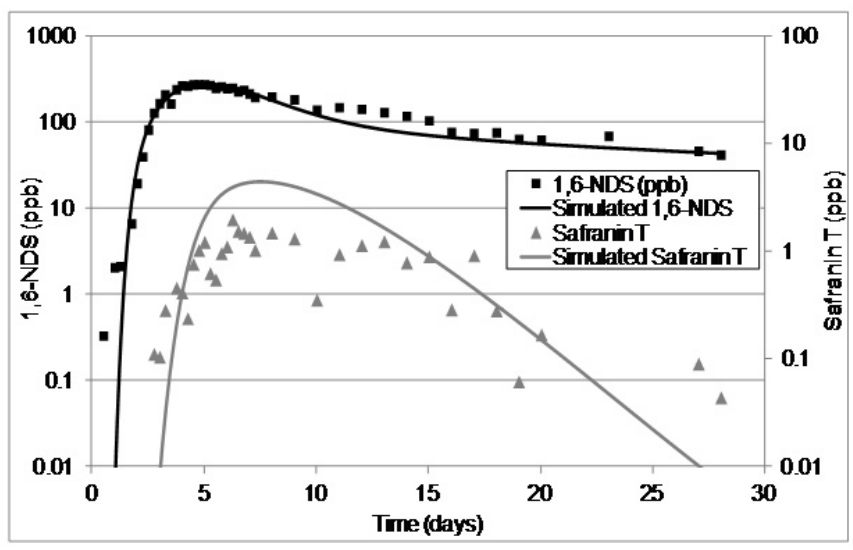

Note that Saf-T concentrations are NOT corrected for thermal decay, but model simulates thermal decay using Arrhenius parameters from laboratory experiments

\section{Model uses MINC feature of TOUGHREACT}

\section{Simulated 2-D Tracer Plumes}
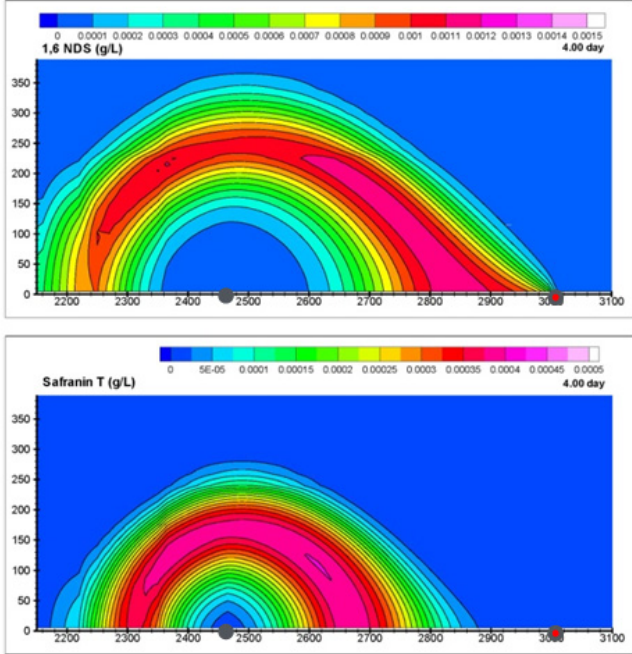

- Injection well

- Production well

Figure 6.9. TOUGHREACT Soda Lake, Nevada tracer test model results.

\subsubsection{Simulated Single-Well Injection/Withdrawal Tests at Soda Lake}

In previous numerical modeling studies, Pruess and Doughty (2010) and Fayer et al. (2009) have demonstrated the potential for using single-well injection/withdrawal tests to characterize fracture surface area in geothermal reservoirs. In this effort we are using the site-specific parameters determined from the two-well tracer test conducted at the Soda Lake site to investigate the potential responses of a single-well injection/withdrawal test conducted at the site under different operational conditions. The goals are to 1) show sensitivity of the test results under different operating conditions to help design a single-well conduct injection/withdrawal testing at the Soda Lake site to provide optimal responses for fracture surface area characterization, 2) help identify beneficial reactive tracer properties for such a test (e.g., thermal decay, sorption), and 3) to address impacts of parameter uncertainty. Two grids were developed during these initial scoping simulations to investigate grid effects on the results: a 2D plan view grid (similar spacing as used in the two-well tracer test) and a 1D radially symmetric grid. Both grids used 10-m spacing near the injection/withdrawal well. Results were similar for the two models; therefore, the results of the $1 \mathrm{D}$ radially symmetric model are reported here, because it was more computationally efficient.

Simulated single-well injection/withdrawal tracer test results are shown in Figure 6.10 using the site parameters from fitting the two-well tracer test, shown in Figure 6.9 and Table 6.8. Given that one of the goals for a single-well injection/withdrawal test is a shorter operational time, the simulated test case consisted of $3 \mathrm{~d}$ of injection, $3 \mathrm{~d}$ of shut-in (no flow), and $3 \mathrm{~d}$ for withdrawal. Two different operational cases are shown in Figure 6.10. The first case uses an initial pulse of tracer during the first hour of the test followed by freshwater injection for the rest of the injection stage (as in the two-well tracer test 
operation). The second case uses a constant tracer injection concentration for the duration of the injection stage but with the same overall mass injected as the first case.

Table 6.8. Summary of parameters for fitting tracer test with TOUGHREACT V1.2. Soda Lake twowell tracer test (conducted fall of 2011, Rose et al. 2012). Simulation: sltwr24, 5/2/2012.

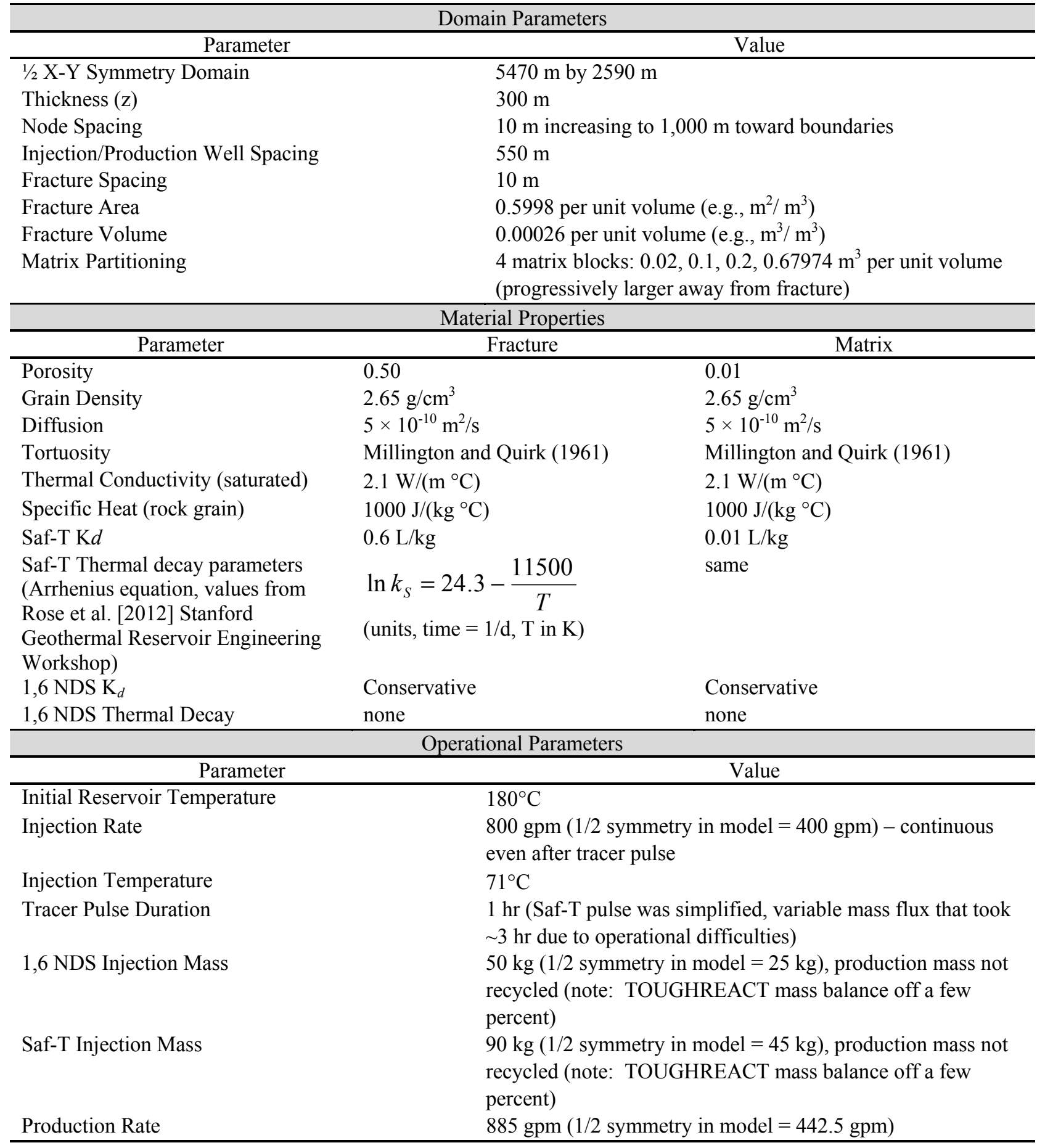


The simulated results in Figure 6.10 illustrate the potential for using single-well tracer tests for surface area interrogation. The differences between the 1,6 NDS and Saf-T concentrations are a function of the SA/V ratio in the system, and the volume in this case is simply the volume of water injected (tracer pulse + fresh). Note that in a porous medium, a fast and reversibly sorbing tracer with little sorption/desorption hysteresis, like Saf-T, would be expected to have a response during the withdrawal period very similar to that of a conservative tracer because the transport of the Saf-T would be retarded by the same amount going into and coming out of the flow system. Such a response would not be very useful for surface area interrogation. Figure 6.10 shows significant differences between the 1,6-NDS and Saf-T because of Saf-T sorption in the matrix after diffusion out of fractures and into the matrix. Under these circumstances, the effective mass transfer coefficient for matrix diffusion is $\frac{\emptyset}{b} \sqrt{D_{m} R_{m}}$, where $\mathrm{R}_{\mathrm{m}}$ is the matrix retardation factor. The value of $\mathrm{R}_{\mathrm{m}}$ for $\mathrm{Saf}-\mathrm{T}$ is large enough that a considerable difference is observed in the responses of the two tracers, which can be exploited to estimate a SA/V ratio $(1 / b)$ provided reasonable estimates of $\phi$ and $D_{m}$ for the tracers are available.

1-hr Tracer Pulse, 3-day Injection

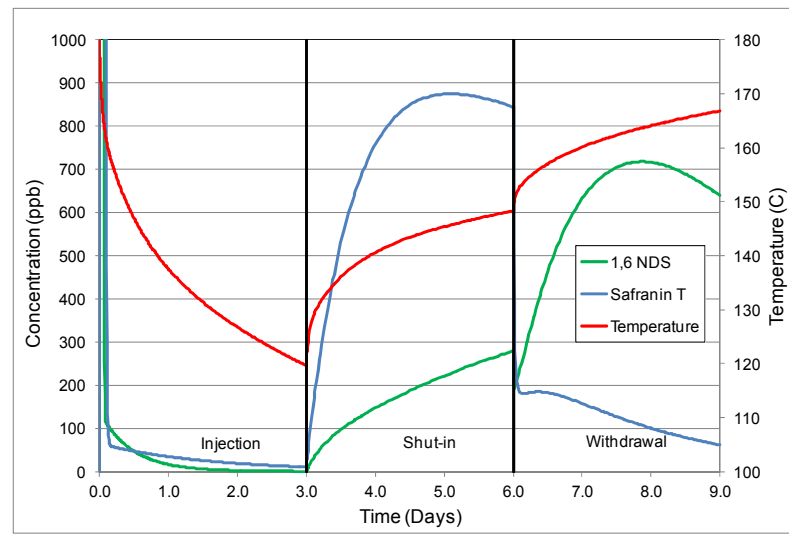

3-day Tracer Pulse (same mass)

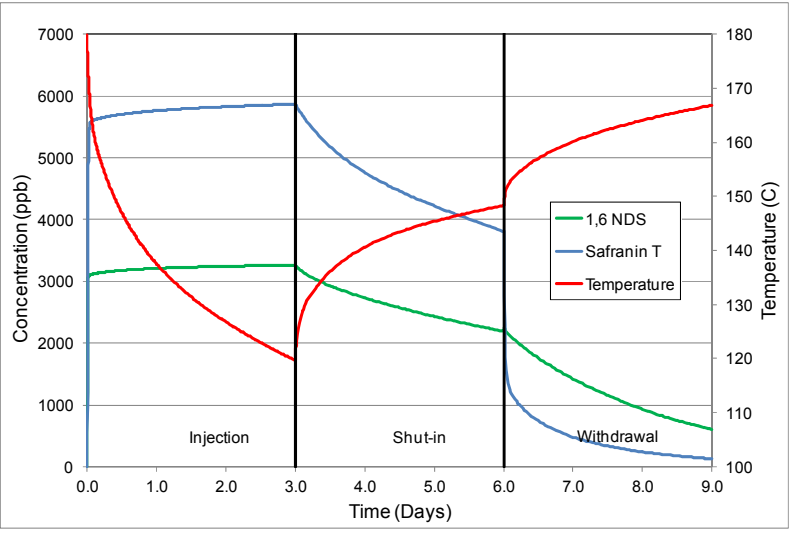

- Use same model parameters as in interwell test simulation (fast, linear, reversible sorption and thermal decay simulated for Safranin-T)

- Differences between 1,6 NDS and Safranin-T are a function of surface area and thermal decay

- Other parameters must be known if surface area is to be estimated (e.g. Safranin-T sorption on matrix and fracture minerals)

Figure 6.10. Simulated single-well tracer tests with a conservative tracer (1,6 NDS) and a reactive tracer (Safranin-T) using the TOUGHREACT code.

Results of additional sensitivity studies for single-well injection/withdrawal tests using the TOUGHREACT code are shown in Figure 6.11. These results show the difference in responses to changes in fracture spacing (i.e., fracture area per unit volume) and fracture volume. Results are shown only for the withdrawal stage using the base case tracer pulse (1-hr tracer pulse shown in Figure 6.10) with the withdrawal concentrations converted to cumulative mass withdrawn. Temperature results show that the withdrawal temperatures were not sensitive to these changes in the fracture volume (e.g., fracture aperture, porosity) but were sensitive to fracture area (e.g., closer fracture spacing). These results were similar to conclusions by Pruess and Doughty (2010). For tracer mass recovery, the cases showed 
differences with changes in either fracture area (e.g., fracture spacing) or fracture volume. Lower tracer recovery was simulated with increased fracture area and higher tracer recovery with greater fracture volume (less relative loss to matrix). Specific tracer recovery responses are site- and tracer-specific. For example, mass recovery for Saf-T is less than the conservative tracer as a function both of sorption and thermal decay.

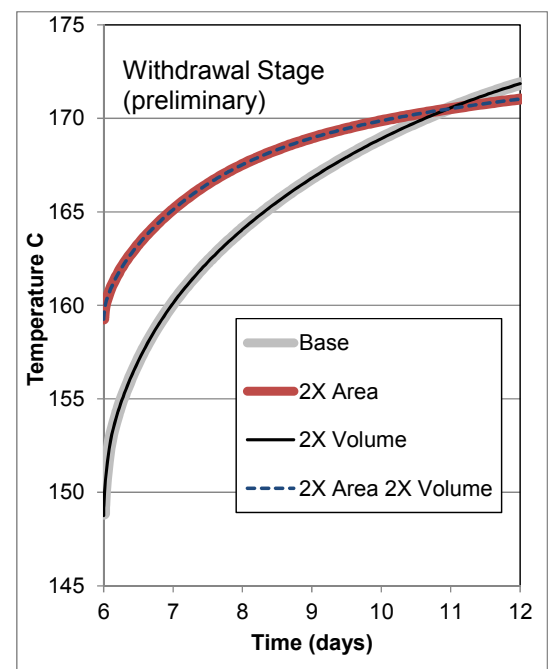

- Higher temperature response to increased fracture density (e.g. closer fracture spacing, increased fracture surface area)

- Similar temperatures for changes only in fracture volume (e.g. aperture, fracture porosity) (see Pruess and Doughty 2010)
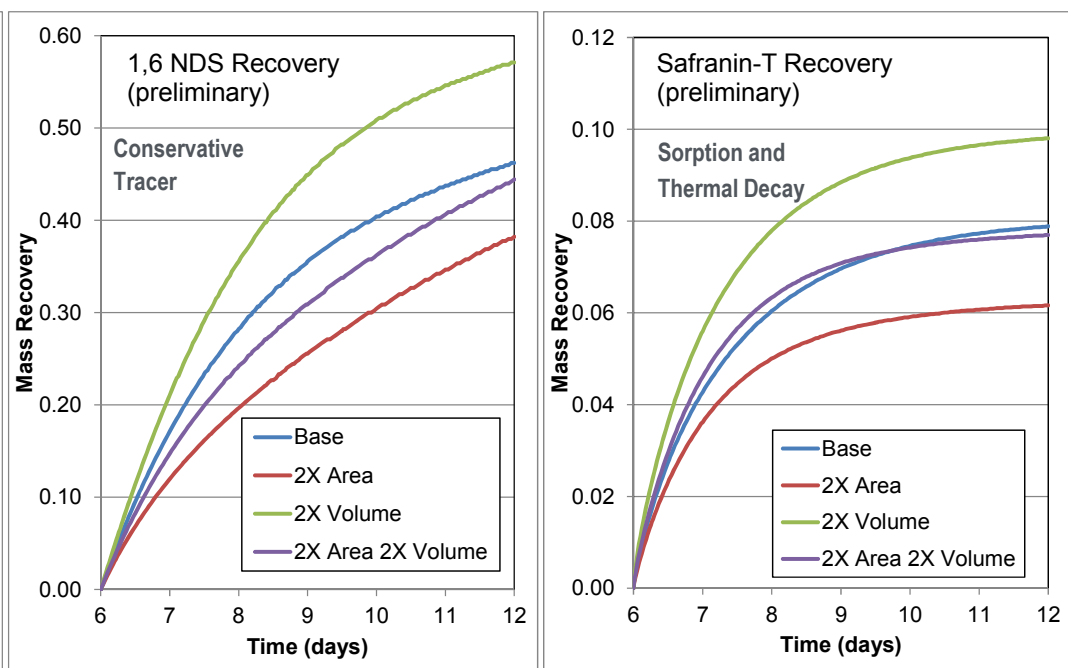

- Lower tracer recovery with increased fracture density

- Higher tracer recovery with greater fracture volume (less relative loss to matrix).

- Responses are site and tracer specific

Figure 6.11. Results of sensitivity studies for single-well injection/withdrawal tests using the TOUGHREACT code.

\subsection{Newberry Crater, Oregon}

PNNL and LANL are collaborating with Dr. Peter Rose at EGI on tracer data interpretation from ongoing laboratory experiments and single-well tracer tests being conducted at the AltaRock Newberry EGS demonstration site. The site description and planning for the EGS test at Newberry Crater in Oregon is described in AltaRock (2011). The site location is shown in Figure 6.12. LANL was involved in the tracer selection and high-temperature laboratory studies of the tracers and main minerals from the site. PNNL has participated in both the tracer test design and field test operations. The EGS demonstration (Petty et al. 2013; Cladouhos et al. 2013) was designed to stimulate three separate zones in a single borehole using a process called hydroshearing. Depth targeted stimulation was accomplished by injecting thermally degrading zonal isolation materials (TZIMs) after each of the stimulation operations to temporarily plug the previously stimulated zone and facilitate stimulation of subsequent depth intervals. Zonal stimulation and tracer injection operations were conducted from October 17 to December 7, 2012. Conservative and reactive tracers were injected into each of the stimulation zones just prior to each of the TZIM injections. The objective of the tracer tests is to estimate the fracture surface area created from the 


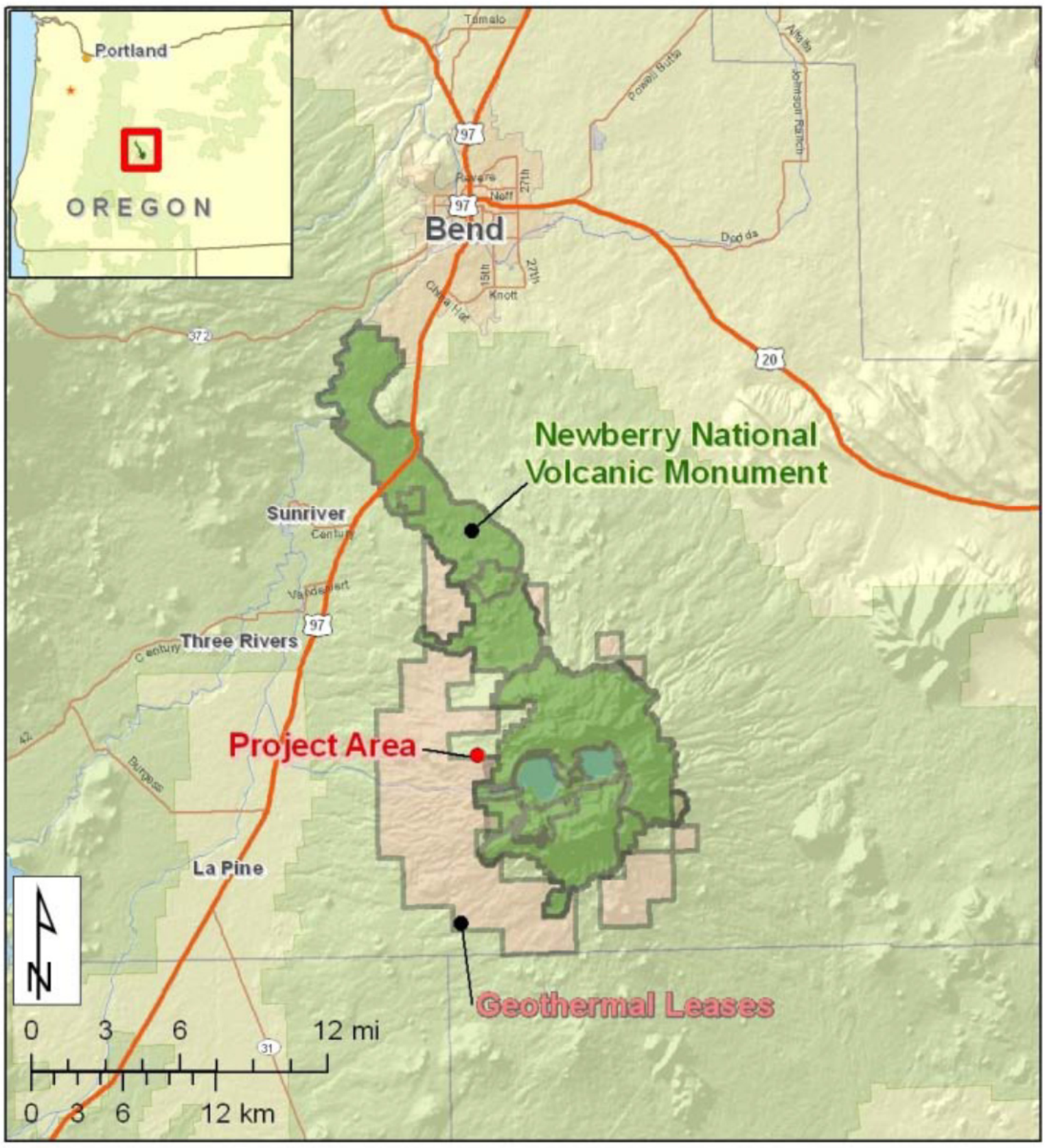

Figure 6.12. Newberry Crater EGS demonstration site showing the Newberry National Volcanic Monument, geothermal leases, and the communities of Bend, Sunriver, Three Rivers, and La Pine (from AltaRock 2011).

stimulations by comparing the tracer returns during flow back. A combination of conservative and reactive tracers were injected into the first and last stimulation zones, while only a conservative tracer was used in the second zone. Different conservative and reactive tracers were used for each zone. Three different naphthalene disulfonate compounds were used for the conservative tracers and lithium and cesium were used for the sorbing tracers. These tracers were selected based on laboratory experiments at LANL and EGI as described by Dean et al. (2013). The flowback phase of the test was delayed until the spring of 2013 because of winter weather conditions and associated access limitations at the site. 
This effort also contributed to the initial scoping design analysis for the tracer tests using simulations with the MULTRAN and TOUGHREACT codes. However, operational constraints of the stimulations and tracer tests changed the tracer test design. Further characterization of the important minerals and tracer interactions at the site are continuing in high-temperature laboratory experiments at EGI and LANL. Cation-exchange models are being refined for analysis of the tracer test results. PNNL also provided technical support for both field test planning and operations. In addition to providing the tracer injection process control and monitoring equipment used in the experiment (see process trailer and mixing tanks in Figure 6.13), PNNL staff took a lead role in systems integration (i.e., between AltaRock's stimulation and diverter injection systems and the tracer injection systems), planning and field implementation, and supported tracer test field operations.

\subsubsection{Laboratory Experiments on Selected Tracers}

Laboratory experiments were conducted to estimate cation-exchange parameters for $\mathrm{Li}$ and $\mathrm{Cs}$ ions to facilitate interpretations of tracer tests at Newberry Crater (Dean et al. 2013). The materials used in the experiments included a crushed $(250-500 \mu \mathrm{m})$ and annealed $\left(450^{\circ} \mathrm{C}\right.$ for $\left.48 \mathrm{hr}\right)$ mineral assemblage representing reservoir fracture coatings comprising roughly equal mass fractions of chlorite, albite, calcite, epidote, and quartz (the epidote fraction was about $18 \mathrm{wt} \%$ compared to $\sim 21 \mathrm{wt} \%$ for the other minerals because of limited quantities of epidote), and a synthetic water having a chemistry matching that of Newberry Crater injection water. All minerals were obtained from Wards Science, except for the quartz, which was an Ottawa (IL) sand obtained from Agsco Corp.

Batch sorption experiments using Li were performed at room temperature to obtain estimates of sitespecific cation-exchange parameters. In addition, flow-through column transport experiments were performed at EGI and LANL to study cation-exchanging tracer transport relative to that of a conservative tracer $(1,5$ naphthalene disulfonate [NS]) as a function of temperature, using a synthetic water matching the chemistry of the injection water at Newberry Crater. The experiments were conducted at temperatures of $125^{\circ} \mathrm{C}, 150^{\circ} \mathrm{C}, 225^{\circ} \mathrm{C}$, and $275^{\circ} \mathrm{C}$. The water used in the higher temperature experiments $\left(225^{\circ} \mathrm{C}\right.$ and $275^{\circ} \mathrm{C}$ ) had potassium salts substituted for magnesium and calcium salts to reduce mineral precipitation at these temperatures (see Table 6.9). This K-substituted water was used in all high-temperature flowthrough experiments because calcite $\left(\mathrm{CaCO}_{3}\right)$ and magnesite $\left(\mathrm{MgCO}_{3}\right)$ were predicted to be significantly supersaturated in the Newberry water at the higher temperatures, and there was a concern that such precipitation could interfere with lithium and cesium transport via co-precipitation reactions and also that it might significantly decrease the permeability of the reactor, possibly leading to plugging. The experiments were performed using the above mineral assemblage at temperatures ranging from $125^{\circ} \mathrm{C}$ to $275^{\circ} \mathrm{C}$. A more detailed description of the materials and methods for the experiment can be found in Dean et al. (2013).

Conservative tracer data from the Newberry laboratory column experiments were fit using the semianalytical model RELAP described in Chapter 4.0 to determine column parameters of residence time $(\tau)$ and Peclet number (Pe). These parameters were then used in MULTRAN (Chapter 5.0) to simulate the cation-exchanging tracer breakthrough curves and to estimate reactive transport parameters for these tracers. The goal in the case of lithium was to simulate the four breakthrough curves obtained at the four different temperatures using a single set of cation-exchange parameters. 

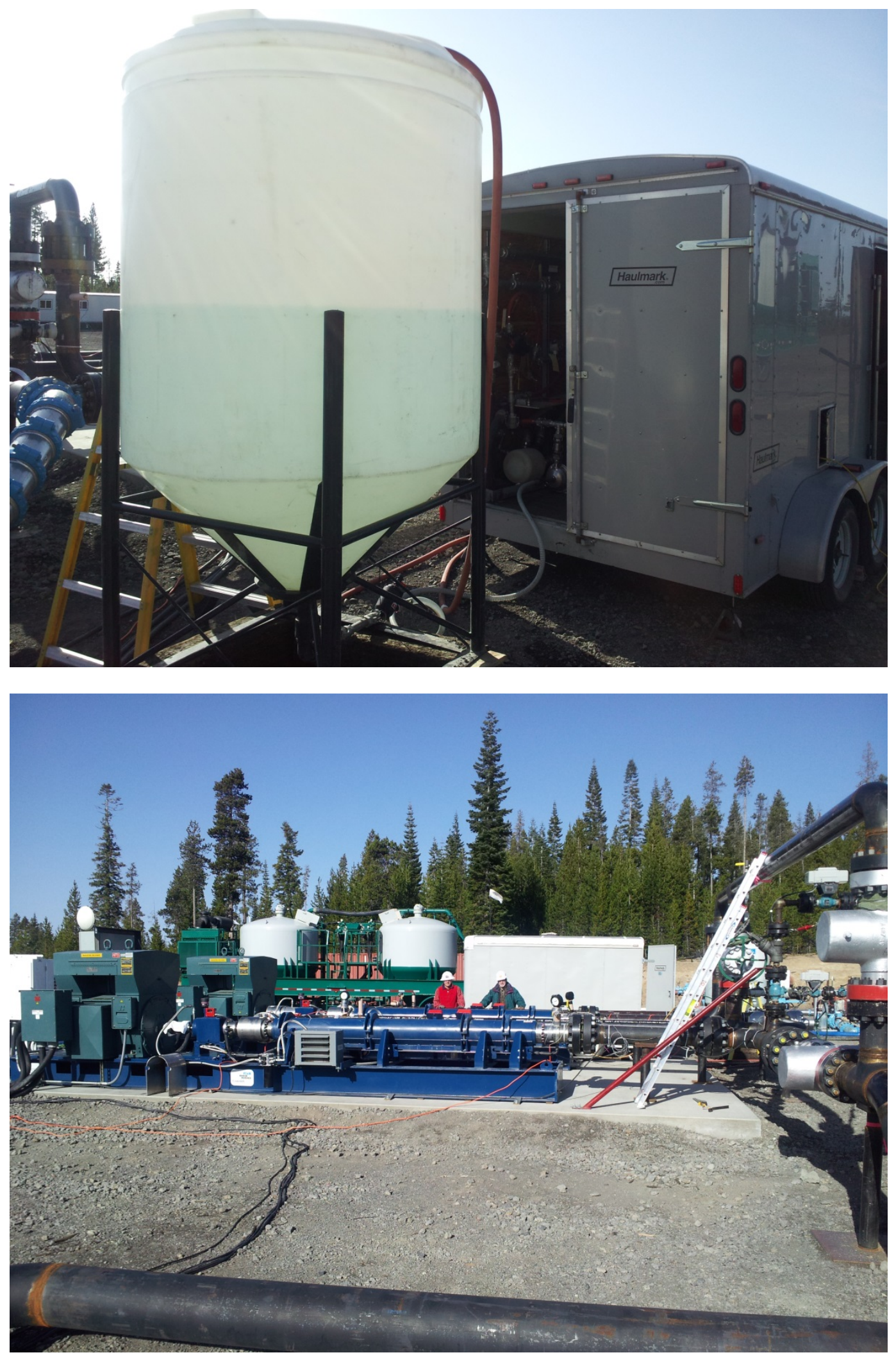

Figure 6.13. PNNL process trailer and mixing tank (top photo) used for tracer tests at Newberry Crater field site (bottom photo). 
Table 6.9. Synthetic water chemistries used in the laboratory experiments.

\begin{tabular}{lrlr}
\hline \multicolumn{2}{c}{ Newberry Crater Synthetic Water } & \multicolumn{2}{c}{ K-Substituted Synthetic Water } \\
\hline \multicolumn{1}{c}{ Chemical } & $(\mathrm{mg} / \mathrm{L})$ & \multicolumn{1}{c}{ Chemical } & $(\mathrm{mg} / \mathrm{L})$ \\
\hline $\mathrm{MgSO}_{4}$ & 3.13 & $\mathrm{~K}_{2} \mathrm{SO}_{4}$ & 4.53 \\
$\mathrm{CaCl}_{2}$ & 5.55 & $\mathrm{KCl}$ & 7.46 \\
$\mathrm{KHCO}_{3}$ & 13.01 & $\mathrm{KHCO}_{3}$ & 13.01 \\
$\mathrm{Mg}\left(\mathrm{HCO}_{3}\right)_{2}$ & 146.74 & $\mathrm{KHCO}_{3}$ & 200.80 \\
$\mathrm{Ca}\left(\mathrm{HCO}_{3}\right)_{2}$ & 68.72 & $\mathrm{KHCO}_{3}$ & 84.89 \\
$\mathrm{NaHCO}_{3}$ & 160.75 & $\mathrm{NaHCO}_{3}$ & 160.75 \\
$\mathrm{H}_{4} \mathrm{SiO}_{4}$ & 96.00 & $\mathrm{H}_{4} \mathrm{SiO}_{4}$ & 96.00 \\
\hline
\end{tabular}

\subsubsection{Room-Temperature Batch Adsorption Experiments}

Figure 6.14 contains the mineral surface areas, estimated CEC values, and average $\mathrm{K}_{d}$ values (partition coefficients, $\mathrm{ml} / \mathrm{g}$ ) of the mineral fractions contained in the mineral assemblage (mix) for $\mathrm{Li}^{+}$ (left) and $\mathrm{Cs}^{+}$(right) using the synthetic Newberry water (without $\mathrm{K}$ substituted for $\mathrm{Mg}$ and $\mathrm{Ca}$ ). The combined fractional surface area of the minerals (sum of the product of fractions and specific surface areas) is twice that measured for the mix, which is most likely due to sample inhomogeneity. The surface areas were measured by Brunauer, Emmett, Teller (BET) gas adsorption isotherm. The combined fractional $\mathrm{Li}^{+} \mathrm{CEC}$ of the minerals (sum of the product of fractions and CECs) is $3.49 \mathrm{meq} / \mathrm{kg}$, slightly lower than that measured in the mix $(4.40 \mathrm{meq} / \mathrm{kg})$. The combined fractional Cs $+\mathrm{CEC}$ of the minerals (sum of the product of fractions and CECs) is $4.86 \mathrm{meq} / \mathrm{kg}$, slightly higher than that measured in the mix $(4.39 \mathrm{meq} / \mathrm{kg})$. Chlorite, having the greatest surface area and CEC, contributes most to CEC in the mix. The CEC measurements were performed by placing the minerals in contact with a $1 \mathrm{M}$ solution of either $\mathrm{Li}^{+}$or $\mathrm{Cs}^{+}$and then measuring the total equivalents of other cations that were displaced from the minerals by the $\mathrm{Li}^{+}$or $\mathrm{Cs}^{+}$.

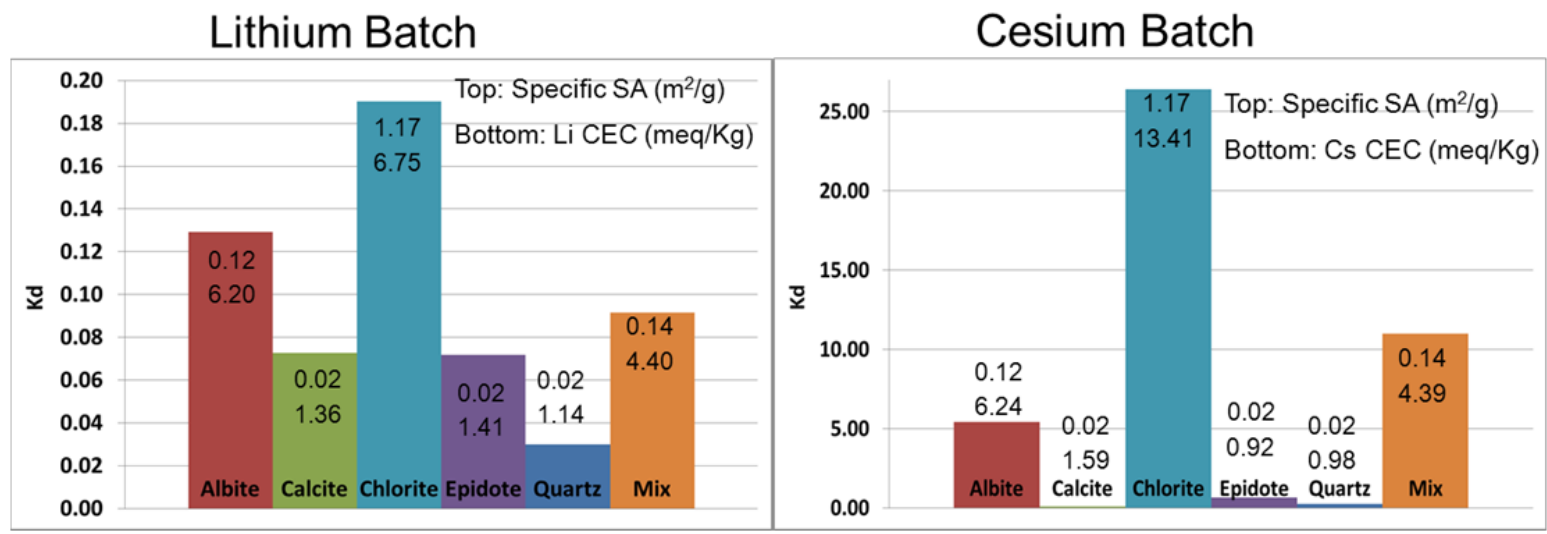

Figure 6.14. Lithium (left) and cesium (right) room temperature batch experimental results with synthetic Newberry Crater water chemistry that does not have K substituted for Ca and $\mathrm{Mg}$.

Additional batch adsorption experiments were performed with the mineral mix using the K-substituted synthetic water of Table 6.7 (the K-substituted water was not used to measure adsorption to the individual minerals). The sorption of $\mathrm{Li}^{+}$(left) and $\mathrm{Cs}^{+}$(right) in the mineral mix using the 
K-substituted water and the water containing $\mathrm{Ca}$ and $\mathrm{Mg}$ is shown in Figure 6.15. $\mathrm{The}^{-{ }^{+}} \mathrm{K}_{d}$ value for the mineral mix in the K-substituted water is slightly higher at 1- and 5-ppm starting concentrations and slightly lower at the 25-ppm starting concentration than in the original Newberry water; both are quite low and the difference is most likely attributable to the experimental error associated with trying to measure small differences in $\mathrm{Li}^{+}$concentrations in solution before and after adsorption. The $\mathrm{Cs}^{+}$sorption to the mineral mix in the K-substituted and original Newberry water is significantly stronger than $\mathrm{Li}^{+}$sorption to the mix and the errors are also smaller because the differences between the solution concentrations before and after sorption were much greater. Also, the K substitution appears to slightly suppress the sorption of $\mathrm{Cs}$ onto the mineral mix, which is consistent with the slightly greater selectivity of most cation-exchange sites for $\mathrm{K}$ than for $\mathrm{Ca}$ and $\mathrm{Mg}$ (Appelo 1996), thus making it slightly more difficult for $\mathrm{Cs}$ to exchange with $\mathrm{K}$ than with $\mathrm{Ca}$ or $\mathrm{Mg}$.

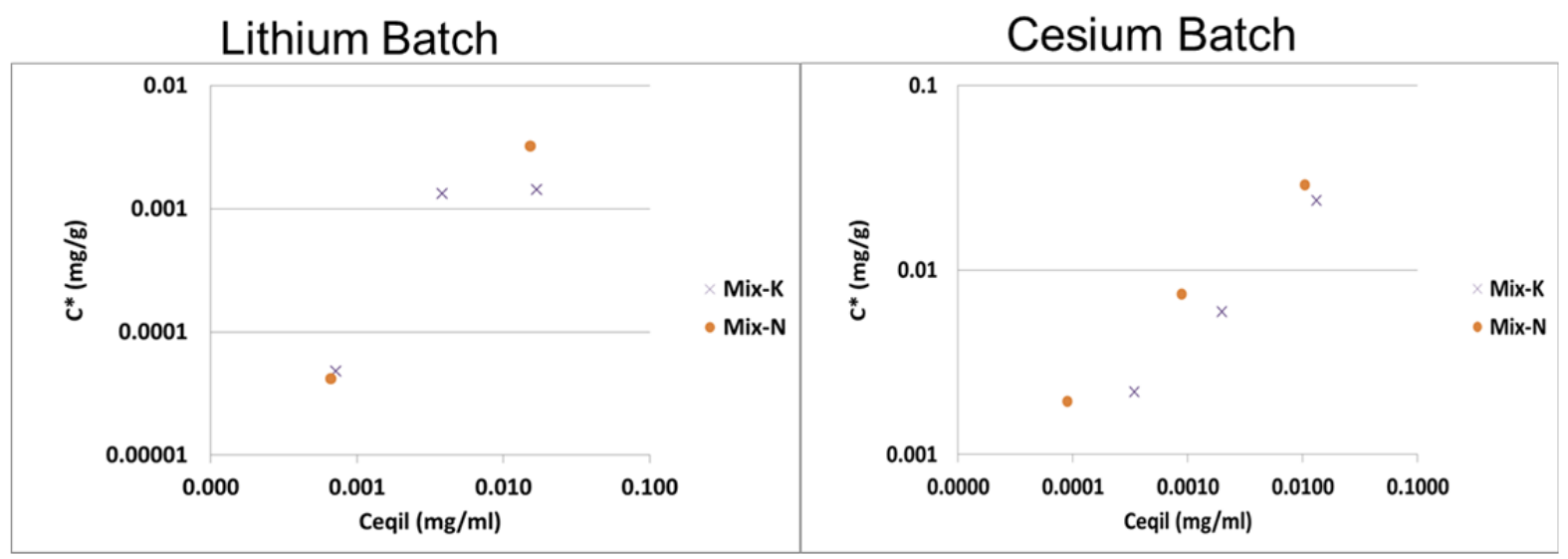

Figure 6.15. Log-log plots of $\mathrm{Li}$ (left) and $\mathrm{Cs}$ (right) batch experiments at $\sim 1 \mathrm{ppm}, \sim 5 \mathrm{ppm}$, and $\sim 25 \mathrm{ppm}$ comparing sorption in K-substituted water in the mix (mix-K) and in the original Newberry water (mix-N).

\subsubsection{Column Transport Experiments and Modeling}

Figure 6.16 shows the EGI and LANL dynamic column flow-through experimental data at various temperatures along with MULTRAN fits to the NS and $\mathrm{Li}^{+}$breathrough curves. The MULTRAN fits were obtained by first fitting the NS data using RELAP to estimate the mean residence time and Peclet number in the columns, and then adjusting cation-exchange parameters as necessary to fit the $\mathrm{Li}^{+}$data. The breakthrough curves of a second conservative tracer, bromide (not shown in Figure 6.15), were confirmed to be nearly identical to those of the NS in the two higher temperature experiments conducted at LANL, which provided a strong indication that there was not a significant amount of matrix diffusion occuring into the mineral grains used in the columns. The mineral grains used in the columns were crushed and sieved to a size range of $250-500 \mu \mathrm{m}$, and they were annealed at $450^{\circ} \mathrm{C}$ for $48 \mathrm{hr}$ to passivate the fresh surfaces created by the crushing process. There was concern that the fresh surfaces might be more reactive than surfaces exposed to fluids or air for a long time in a reservoir, and they would thus provide nonrepresentative results in the column experiments. An unintentional consequence of the annealing treatment was that it oxidized minor amounts of iron present in the chlorite, but subsequent batch adsorption experiments on the original unannealed chlorite, the oxidized annealed chlorite, and 
some of the oxidized chlorite that was re-reduced in a gas chromatography furnace under an $\mathrm{Ar} / \mathrm{H}_{2}$ atmosphere, confirmed that the oxidized and re-reduced chlorite behaved essentially the same with respect to $\mathrm{Li}^{+}$adsorption.
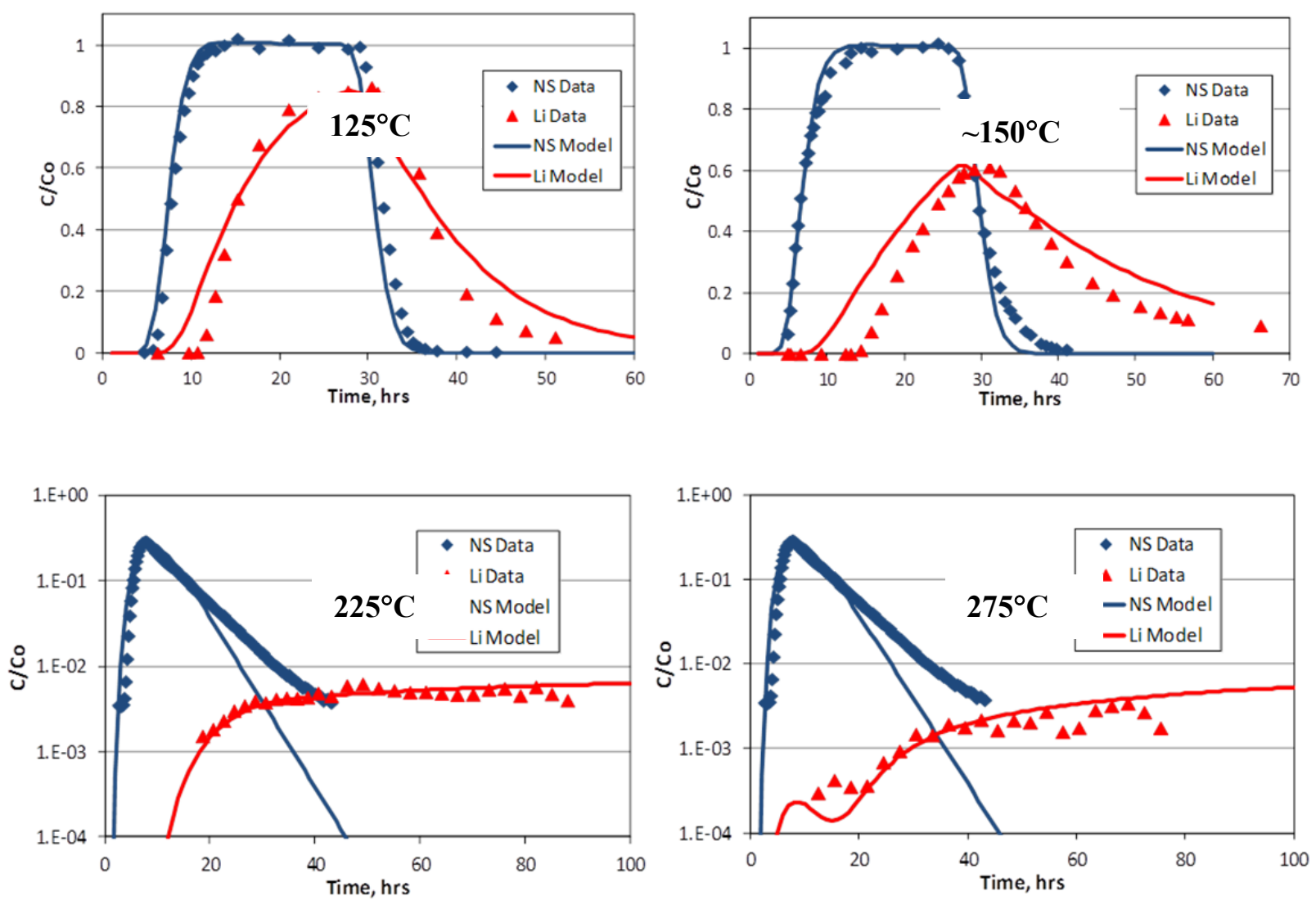

Figure 6.16. $\mathrm{NS}$ and $\mathrm{Li}^{+}$breakthrough curves and model fits (normalized concentration vs. time) in laboratory column experiments. Upper left: EGI T $=125^{\circ} \mathrm{C}, \sim 8$-hr residence time, $\sim 22-\mathrm{hr}$ tracer pulse; upper right: $\mathrm{EGI} \mathrm{T}=\sim 150^{\circ} \mathrm{C}, \sim 8$-hr residence time, $\sim 22-\mathrm{hr}$ tracer pulse; lower left: LANL $\mathrm{T}=225^{\circ} \mathrm{C}, \sim 10$-hr residence time, $\sim 2.5$-hr tracer pulse; lower right: LANL $\mathrm{T}=275^{\circ} \mathrm{C}, \sim 10$-hr residence time, $\sim 2.5$-hr tracer pulse.

The NS tracer breakthrough curves (Figure 6.16) were reproducible at different temperatures in both the LANL and EGI experiments (i.e., the curves were nearly identical when run in the same column under the same flow conditions, but at different temperatures). However, the residence times and tracer pulse times were quite different in the LANL ( $\sim 10-\mathrm{hr}$ residence time, $\sim 2.5-\mathrm{hr}$ tracer pulse) and EGI ( $\sim 8-\mathrm{hr}$ residence time, $\sim 22-\mathrm{hr}$ tracer pulse) experiments. The increased $\mathrm{Li}^{+}$retardation with increased temperature (note the shift to log concentration scales at the two higher temperaures in Figure 6.15) was unexpected and indicates a relatively strong temperature dependence of $\mathrm{Li}^{+}$cation-exchange onto the minerals.

$\mathrm{The} \mathrm{Li}^{+}$breakthrough curves were fit by trial and error using MULTRAN with the constraint that the physical and chemical properties of the grains had to be the same in all the column experiments.

However, the selectivity coefficient $K_{N a / L i}$ (see Equation (5.2)) was allowed to vary because it is expected to have a temperature dependence (Gaines and Henry 1955; Kraus and Raridon 1959). It was found that 
the $\mathrm{Li}^{+}$data from the higher temperature experiments could be fit only by assuming a thin porous layer on the surfaces of the grains that provided access to cation-exchange surfaces by diffusion (in addition to a small amount of exchange on the surfaces that did not require diffusive access). This result is consistent with the "rim-zone" model interpretation of the Soda Lake adsorbing tracer data in Section 6.1.1. In the case of the laboratory experiments involving the crushed minerals, the rim zone would amount to a narrow boundary layer on surfaces of the minerals, as depicted schematically in Figure 6.17.

The cation-exchange and diffusion parameters yielding good fits to the $\mathrm{Li}^{+}$data are listed in Table 6.10. The parameters for the rim zone (rim thickness $=0.04 \mathrm{~cm}$ [with a flowing zone half-thickness of $0.05 \mathrm{~cm}$ ], rim porosity $=0.0012$, and a CEC of $0.046 \mathrm{eq} / \mathrm{kg}$ ) were used to represent the properties of the marix in the single-well tracer test simulations of Section 5.4. Figure 6.18 shows the temperature dependence of selectivity coefficient $K_{N a / L i}$ expressed as a plot of $\ln K_{N a / L i}$ vs. $1 / T\left({ }^{\circ} \mathrm{K}\right)$. The linearity of this plot is consistent with Arrhenius-type behavior for the selectivity coefficient. It was not necessary to adjust the selectivity coefficients of any of the other cations in the experiments conducted at different temperatures to achieve good simultaneous fits to the $\mathrm{Li}^{+}$breakthrough curves. The temperature dependence of $K_{\mathrm{Na} / L i}$ shown in Figure 6.18 was used in the simulations of single-well tracer tests at different temperatures in Section 5.4.

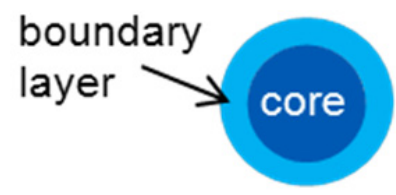

Figure 6.17. Schematic illustration of rim zone on grains in laboratory column experiments.

Table 6.10. Model parameters used in MULTRAN simulations of laboratory column experiments (Figure 6.16).

\begin{tabular}{ll}
\hline Half-aperture (open spaces between grains) & $0.05 \mathrm{~cm}$ \\
Column porosity & $0.4(40 \%)$ \\
Cation-exchange capacity on grain surfaces & $0.0011 \mathrm{eq} / \mathrm{kg}$ \\
Thickness of rim-zone layer on grains & $0.04 \mathrm{~cm}$ \\
Porosity in rim zone & 0.0012 \\
Cation-exchange capacity in rim zone & $0.046 \mathrm{eq} / \mathrm{kg}$ \\
Selectivity coefficient, $K_{N a / L i}$ & Temperature dependent as shown in Figure 6.17 \\
Tracer matrix diffusion coefficients & $2.5 \times 10^{-7} \mathrm{~cm}^{2} / \mathrm{s}$ at $25^{\circ} \mathrm{C}$ for naphthalene sulf. \\
& $6.7 \times 10^{-7} \mathrm{~cm}^{2} / \mathrm{s}$ at $25^{\circ} \mathrm{C}$ for lithium \\
Rock and water properties (density and thermal) & Corresponding to basalt and dilute water \\
\hline
\end{tabular}




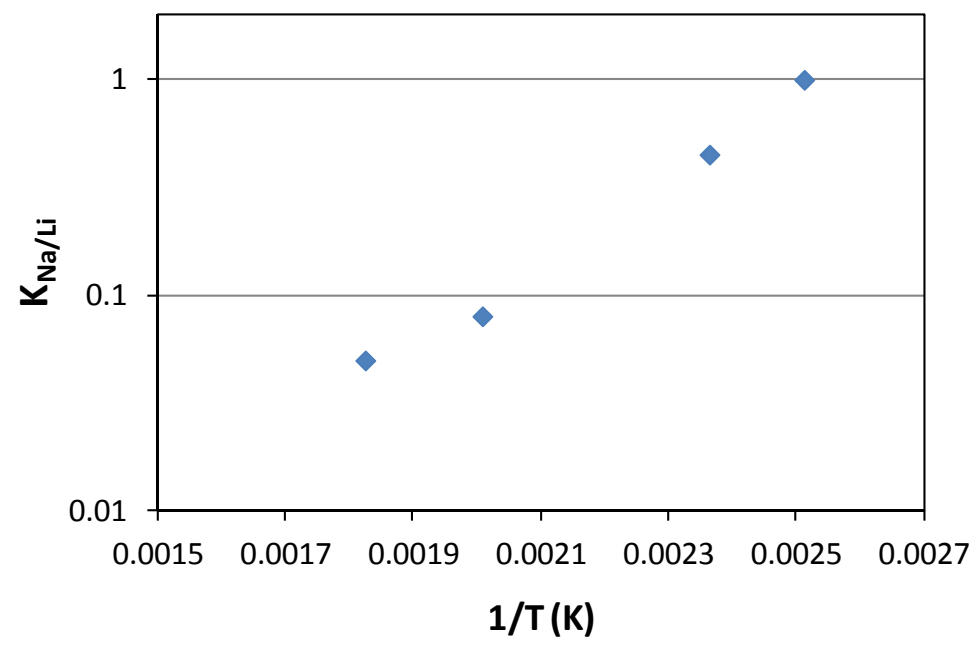

Figure 6.18. Temperature dependence of selectivity coefficient $K_{N a / L i}$ used to simultaneously fit the lithium breakthrough curves of Figure 6.15. $K_{N a / L i}$ was the only adjustable parameter used to fit breakthrough curves obtained at the different temperatures.

Figure 6.19 shows the NS and $\mathrm{Cs}^{+}$experimental data and model fits from a column experiment conducted at $225^{\circ} \mathrm{C}$ at LANL along with the $\mathrm{Li}^{+}$data obtained in the same column at the same temperature. A minor modification of the $\mathrm{CEC}$ value of the mineral mix was required to fit the $\mathrm{Cs}^{+}$data. This may be attributable to mineral alteration during the $275^{\circ} \mathrm{C} \mathrm{Li}^{+}$experiment before the temperature was decreased to $225^{\circ} \mathrm{C}$ for the $\mathrm{Cs}^{+}$experiment. Cesium is a stronger cation-exchanger than $\mathrm{Li}$ at room temperature; therefore, it was expected that itwould be more retarded in the column experiment than Li. However, it is apparent in Figure 6.19 that the Cs was slightly less retarded than $\mathrm{Li}$ at $225^{\circ} \mathrm{C}$. A possible explanation for this result is that higher temperatures tend to disrupt the waters of hydration that surround alkali metals (Li, Na, K, Cs) at lower temperatures (Gast and Klobe 1971). Being the smallest monovalent alkali metal cation (highest charge to size ratio), Li tends to be the most hydrated of the alkali metals at low temperatures, which is believed to be why it is the weakest exchanger of this group of cations. In contrast, Cs exchanges very strongly at low temperatures because it is poorly hydrated. However, as temperatures increase, thermal energy disrupts the waters of hydration surrounding the cations, and this effect is largest for $\mathrm{Li}$. This effect would explain both the observed temperature dependence of $\mathrm{Li}^{+}$exchange in the column experiments (Figure 6.16) and the greater reactivity of $\mathrm{Li}^{+}$than $\mathrm{Cs}^{+}$at $225^{\circ} \mathrm{C}$.

As Figure 6.19 shows, a higher ionic strength flushing solution was inadvertently introduced after approximately $80 \mathrm{hr}$ in the $\mathrm{Cs}^{+}$experiment, and this change in flushing solution chemistry resulted in a subsequent increase in $\mathrm{Cs}^{+}$concentrations that was qualitatively reproduced by MULTRAN. This inadvertant increase in ionic strength of the flushing solution provided a validation test of sorts for MULTRAN.

Figure 6.20 shows $\mathrm{Li}^{+}$breakthrough curves from MULTRAN simulations assuming that all experiments were conducted with 2.5 -hr tracer pulses and $10-\mathrm{hr}$ residence time (the LANL experimental conditions). It is apparent that the $\mathrm{Li}^{+}$breakthrough at $25^{\circ} \mathrm{C}$, predicted using a retardation factor of 1.3 based on the batch sorption data, significantly over-predicts the Li breakthrough at higher temperatures. 
These simulations illustrate the effects of the extreme temperature dependence of $\mathrm{Li}^{+}$exchange in the column transport experiments. Clearly, it would be important to account for this temperature dependence in any field experiment in which $\mathrm{Li}^{+}$is used as a reactive tracer.

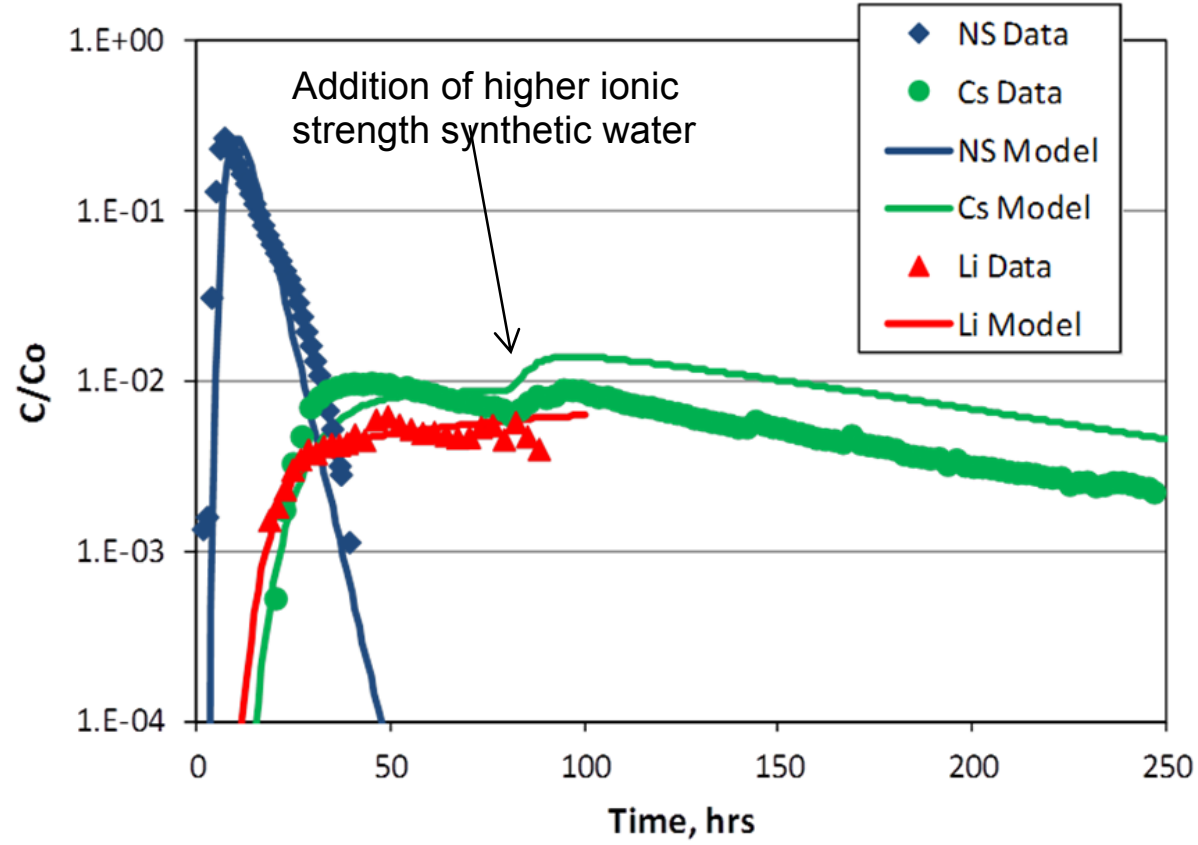

Figure 6.19. $\mathrm{NS}, \mathrm{Li}+$, and $\mathrm{Cs}+$ breakthrough curves (normalized concentration vs. time) and MULTRAN fits at $225^{\circ} \mathrm{C}$ in the LANL columns ( $\sim 10$-hr residence time, $\sim 2.5$-hr tracer pulse).

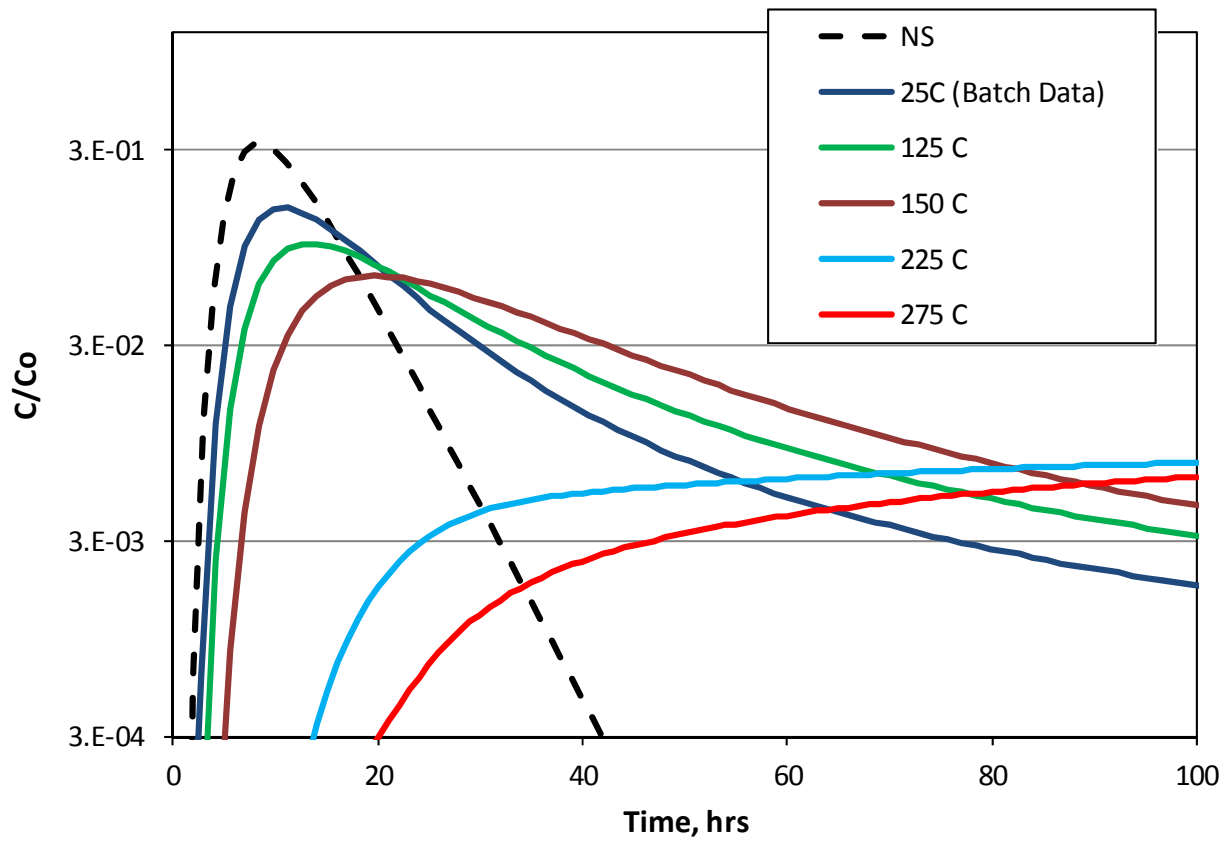

Figure 6.20. $\mathrm{NS}$ and $\mathrm{Li}^{+}$breakthrough simulations at temperatures ranging from $25^{\circ} \mathrm{C}$ to $225^{\circ} \mathrm{C}$ assuming LANL column experimental conditions $(\sim 10-\mathrm{hr}$ residence time, $\sim 2.5-\mathrm{hr}$ tracer pulse). 


\subsubsection{Development of TOUGHREACT Tracer Simulations for Newberry Crater EGS Demonstration}

TOUGHREACT numerical models, which were based on a nominal test design and a TOUGHREACT modeling framework, were used to help plan the Newberry Crater tracer tests. These models were also used for interpretation of the Newberry tracer test results. A 2D radially symmetric grid was constructed for the open borehole section (1066 m thick) for the EGS demonstration along with hydrostratigraphic layering based on preliminary data provided by AltraRock Energy, Inc. The TOUGHREACT models implemented the opening and closing of three intervals in the open borehole section to represent the three sets of stimulation and diverter injections that were planned. The opening and closing of these intervals were simulated through imposed step permeability changes in the model domain and not through simulation of the geomechanics of the stimulations and diverter injections. The locations of these stimulation zones were based on pretest predictions by AltaRock Energy, Inc. of where the stimulation zones might occur. The predictions were based on drilling mud loss records and observed lithology. The modeling framework for the Newberry Crater tracer tests consists of a large series of TOUGHREACT restart runs to track the various operational stages (i.e., three cycles of stimulation, tracer injection, and diverter injection, shut-in of the well, and a final flowback stage).

A limitation in the initial TOUGHREACT scoping-level modeling was the use of $\mathrm{K}_{d}$ linear sorption for the cations (i.e., $\mathrm{Li}^{+}$and $\mathrm{Cs}^{+}$). It was determined, based on laboratory experiments (see Section 6.2.1), that a more realistic method for simulating the cation tracer transport was to implement cation-exchange reactions into the TOUGHREACT simulations. The setup and testing of cation-exchange reaction networks in TOUGHREACT using column experiments conducted by EGI and LANL are described below. For use in interpretation of the Newberry Crater tracer tests (results expected in spring 2013), the TOUGHREACT modeling framework described in the previous paragraph will need to be updated to include the cation-exchange reaction network.

\subsubsection{Cation Exchange Reactions of Trimix Experiments using TOUGHREACT V2.0}

Work was begun to implement the cation-exchange reactions into the TOUGHREACT tracer simulations. For the initial development and to familiarize ourselves with the TOUGHREACT cationexchange parameters we focused on simulating the four separate trimix column experiments conducted in September and October 2012 by EGI. These experiments included two $\mathrm{LiBr}$ and two $\mathrm{CsCO}_{2} \mathrm{H}$ sorbing tracer injections, which also included conservative tracers (1,5 NDS). The temperatures of these experiments were approximately $120^{\circ} \mathrm{C}$. Details of these laboratory experiments are provided by Rose et al. (2013).

The column transport experiments were conducted using a mix of ground calcite, albite, and quartz sand grains packed in a 20-in.-long, 1-in.-diameter stainless steel column. Assuming a mean particle density of $2.65 \mathrm{~g} / \mathrm{cm}^{3}$ and a measured bulk density of $1.35 \mathrm{~g} / \mathrm{cm}^{3}$ provided a porosity of $49 \%$. A groundwater analogue was prepared to mirror ion concentrations found at pad 29 (a well in the upper aquifer at the Newberry Crater site that was used for water supply in the field tracer tests), but it was modified slightly after initial laboratory tests revealed large variations in effluent $\mathrm{pH}$ and pressure. Tracer solutes were then introduced as either $\mathrm{LiBr}$ or $\mathrm{CsCO}_{2} \mathrm{H}$ to study the retardation of cations through the column. The inlet concentrations used in the experiment can be seen in Table 6.11. The column was flushed with tracer-free groundwater to establish initial conditions before introducing tracer-containing groundwater. The temperatures, pulse lengths, and rates are listed in Table 6.12. 
Table 6.11. Concentrations of simulated groundwater (pad 29) and tracers used in trimix experiments.

\begin{tabular}{lccccccccccc}
\hline \multicolumn{1}{c}{ Species } & $\mathrm{Ca}$ & $\mathrm{Mg}$ & $\mathrm{Na}$ & $\mathrm{K}$ & $\mathrm{HCO}_{3}$ & $\mathrm{SO}_{4}$ & $\mathrm{Cl}$ & $\mathrm{Li}$ & $\mathrm{Cs}$ & $\mathrm{Br}$ & $1,5-\mathrm{NDS}$ \\
\hline $\begin{array}{l}\text { Experimental } \\
\text { Concentrations }[\mathrm{mM}]\end{array}$ & 0.2500 & 0.2593 & 0.2348 & 0.1205 & 0.5750 & 0.1366 & 0.3836 & 1.2 & 1.2 & 1.2 & $0.03-4.7$ \\
\hline
\end{tabular}

Table 6.12. Pulse lengths and rates used in trimix experiments.

\begin{tabular}{lcccc}
\hline & $\mathrm{Li}$ & $\mathrm{Li}$ & $\mathrm{Cs}$ & $\mathrm{Cs}$ \\
& $9 / 12$ & $9 / 24$ & $9 / 26$ & $10 / 8$ \\
\hline Temperature $\left({ }^{\circ} \mathrm{C}\right)$ & 120.2 & 119.8 & 119.4 & 120.0 \\
Pulse Length $(\mathrm{min})$ & 142.0 & 136.0 & 45.8 & 130.0 \\
Pulse rate $(\mathrm{ml} / \mathrm{min})$ & 2.060 & 2.050 & 2.063 & 2.080 \\
\hline
\end{tabular}

TOUGHREACT V2.0 was used to build 1D simulations of these column tests. A 2-mm-node spacing was selected to match the small dispersion measured in conservative tracer breakthrough curves. Cation-exchange reactions were used to simulate the major cations and the $\mathrm{Li}$ and $\mathrm{Cs}$ tracer pulses.

The retardation of the cation breakthrough is determined by the concentration of exchange sites in the solid media and the varied magnitudes of selectivity for individual cations, which is analogous to an ionic-strength dependent rate constant. The CEC represents the potential number of cations that can be accommodated by the media interlayers, and is measured in charge equivalents per mass or volume of solid media. The actual affinity of the media for the particular cations is seen in the selectivity coefficient $\mathrm{K}_{\mathrm{ij}}^{*}$ and is dependent on the activities of species $i$ and $j$ both in solution and in the interlayers. Interlayer activities in TOUGHREACT are defined using the Gaines-Thomas convention, which approximates activities using the fraction of total available sites. TOUGHREACT incorporates these two aspects of cation exchange in the chemical.inp file through the CEC (measured in meq/100 g media) and the dimensionless exchange coefficients for each cation (relative to $\mathrm{Na}^{+}$).

The TOUGHREACT simulations of the CEC reactions for the trimix experiments are preliminary and the focus was shifted to the higher temperature pentamix experiments because those results are closer to the field conditions (mineralogy and temperature) and were significantly different from the trimix experimental results (i.e., $\mathrm{Li}^{+}$adsorption was higher than $\mathrm{Cs}^{+}$in the pentamix experiments as discussed in Section 6.2.1). Selected results of these preliminary simulations are provided in Figure 6.21 for the $\mathrm{Li}^{+}$ tracer experiments. Preliminary TOUGHREACT model fits of the $\mathrm{Cs}^{+}$tracer experiment were not completed before the emphasis was shifted to the pentamix experiments. 

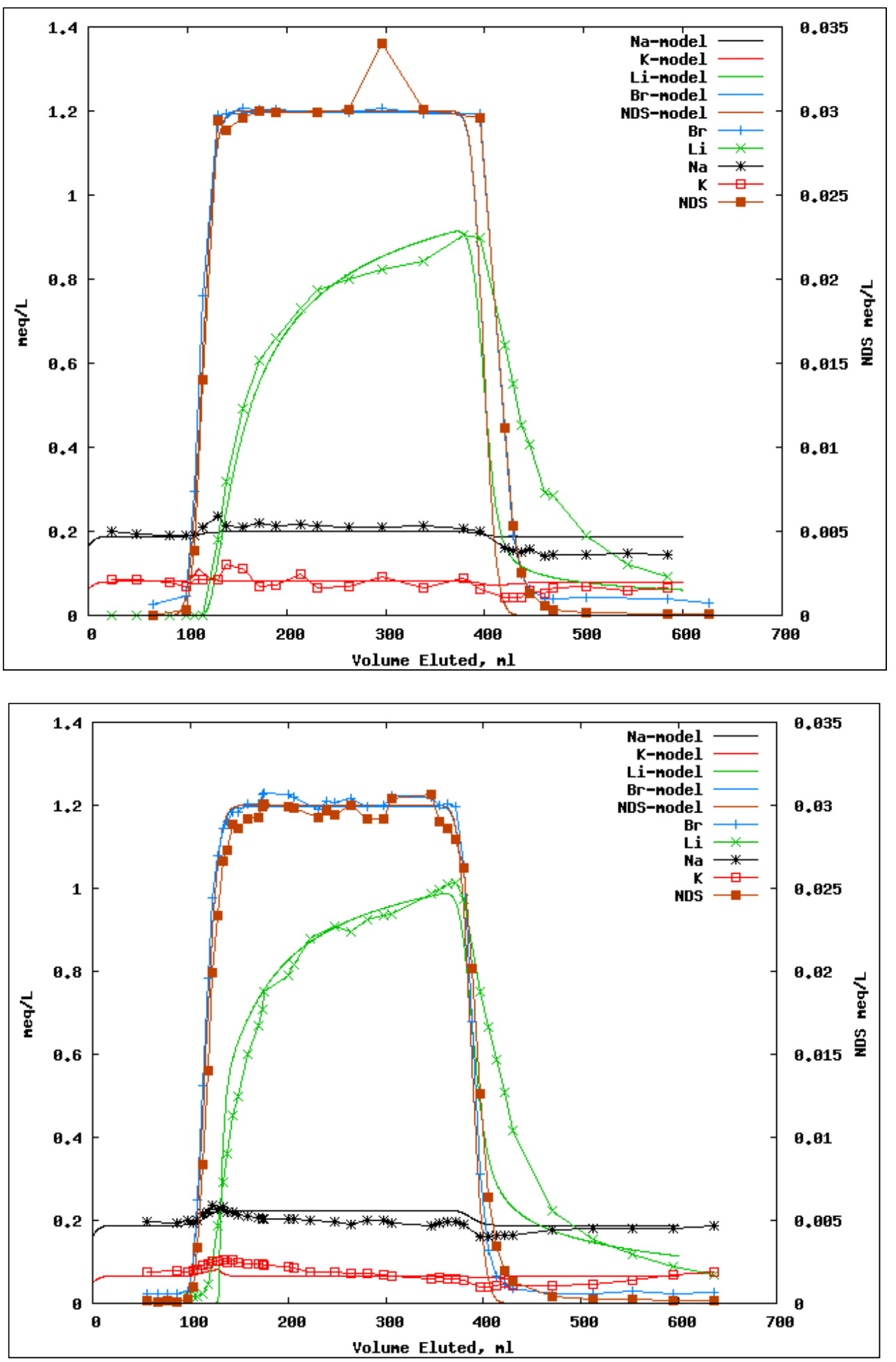

Figure 6.21. Preliminary TOUGHREACT simulations of the $\mathrm{LiBr}$ Trimix experiments using a cation exchange reaction network. Top: 9/12/2012 LiBr experiment. Bottom: 9/24/2012 LiBr experiment. 


\subsubsection{Cation Exchange Reactions of Pentamix Experiments using TOUGHREACT V2.0}

Preliminary TOUGHREACT simulations were developed for fitting the $\mathrm{Li}^{+}$tracer pentamix experiments described in Section 6.2.1 and Dean et al. (2013) with a cation-exchange reaction network. The main differences between the pentamix experiments and the trimix experiments was the mineralogy, water chemistry, and temperature. The pentamix mineral assemblage was selected to represent reservoir fracture coatings and consists roughly equal mass fractions of chlorite, albite, calcite, epidote, and quartz. The water chemistry used K-substituted synthetic groundwater as shown in Table 6.9. Temperatures for the pentamix experiments were run over a wider range of temperatures $\left(125^{\circ} \mathrm{C}, 150^{\circ} \mathrm{C}, 225^{\circ} \mathrm{C}\right.$, and $275^{\circ} \mathrm{C}$ ). As previously discussed, unexpectedly the $\mathrm{Li}^{+}$sorbed more strongly than $\mathrm{Cs}^{+}$in the hightemperature pentamix experiments (the reverse of what was seen in the trimix experiments at lower temperature and with different mineralogy). Figure 6.16 and Figure 6.18 show an increase in $\mathrm{Li}^{+}$sorption in these experiments with increasing temperature.

TOUGHREACT V2.0 was used to build 1D simulations for selected pentamix column tests. A 5 -mm-node spacing was selected to match the small dispersion measured in conservative tracer breakthrough curves. The column used in these tests had a 5.08-cm (2-in) internal diameter and 27.94-cm (11-in) total length, but the bottom $2.54 \mathrm{~cm}$ (1 in.) of the column was rounded. The 1D model grid did not represent the rounded bottom (i.e., constant diameter) but was constructed to preserve the total column volume of $549 \mathrm{~cm}^{3}$. A total porosity of $40 \%$ was specified as described in Section 6.2.1.2. Cation-exchange reactions were used to simulate the major cations and the $\mathrm{Li}^{+}$and $\mathrm{Cs}^{+}$tracer pulses. The MINC option in TOUGHREACT was used to simulate diffusion and cation-exchange between the pore space and the rim zone' on grains (see Figure 6.16). Similarly, the dual-porosity option was used to represent the 'rim zone' in the MULTRAN simulations described in Section 6.2.1.2. Figure 6.22 shows a preliminary example simulation fit of the $\mathrm{Li}^{+}$experiment run at $225^{\circ} \mathrm{C}$.

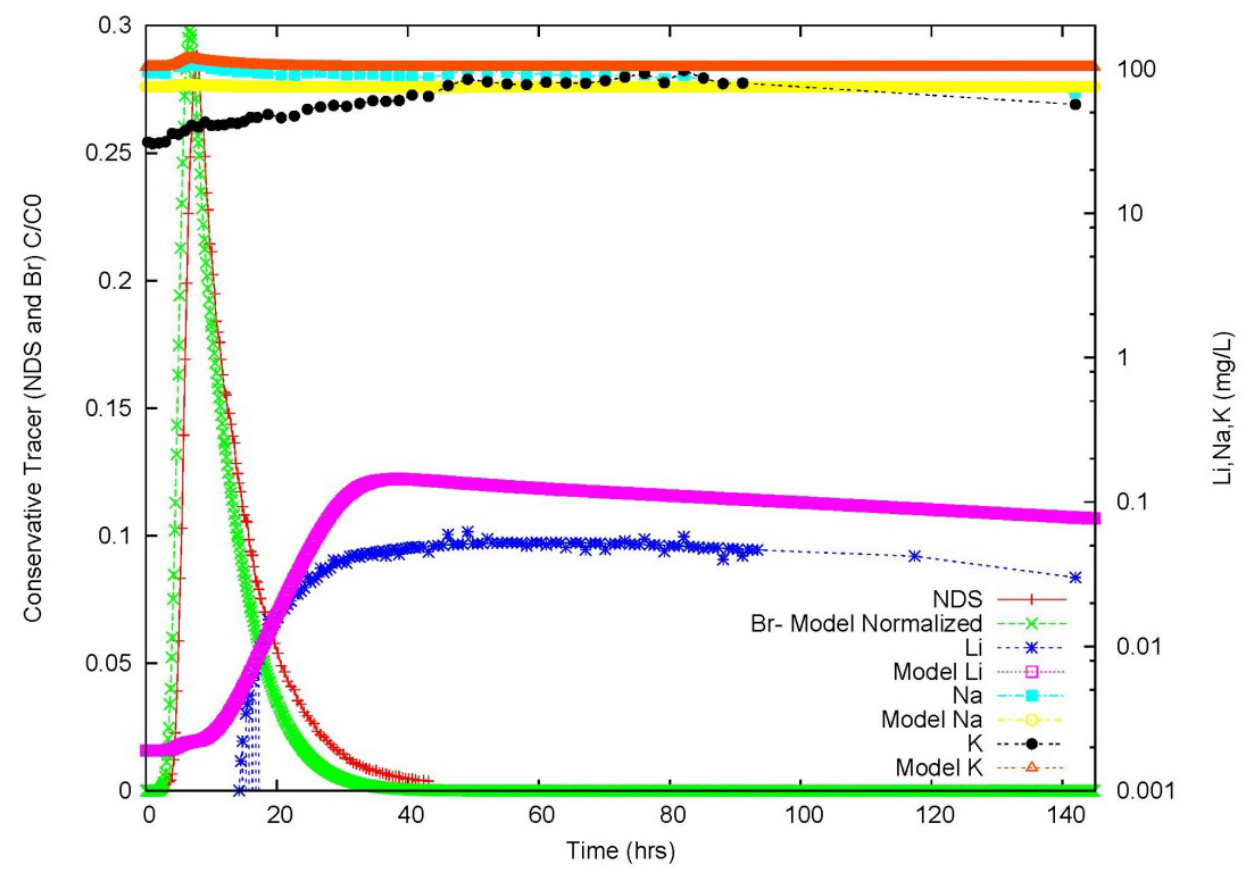

Figure 6.22. Preliminary example TOUGHREACT simulation of the pentamix $\mathrm{Li}+$ experiment at $225^{\circ} \mathrm{C}$ using a cation-exchange reaction network. 


\subsubsection{Tracer Test Operational Support}

PNNL staff took a lead role in the tracer injection field operations at the Newberry Crater EGS demonstration site. PNNL supplied tracer injection equipment and the field expertise needed integrate the tracer injection systems with AltaRock's stimulation and diverter injection systems. Equipment provided by PNNL included 1) the tanks and pumps that were used to prepare and deliver the tracer solutions and 2) a process trailer that contained the process control and monitoring equipment used to conduct the three tracer injections (see Figure 6.13). The process trailer included flow control and diversion valves, pressure gauges, and turbine flowmeters that were used to measure and record the tracer feed rate. All process data were continuously recorded using a data logger.

\subsubsection{Tracer Test Operations}

Three injection/backflow tracer tests were initiated at the Newberry EGS site for the purpose of characterizing the newly created fracture surface area during a multistage hydraulic stimulation experiment. The surface area estimation is based upon the flow of cation-exchanging alkali-metal-ion tracers relative to that of conservative tracers as the tracers are injected into and subsequently produced from the near-wellbore formation. The flow of the reversibly exchanging cation tracers is retarded relative to that of a nonsorbing tracer and this retardation can be used in combination with calibrated numerical models to constrain the contacted fracture-surface area. In support of this project, laboratory sorption experiments were conducted using the cation-exchange tracers $\mathrm{Li}$ and $\mathrm{Cs}$ at elevated temperature and the altered minerals observed to line fracture surfaces at depth in Newberry Crater wells (Dean et al. 2013).

Conservative and reactive tracers were injected in three distinct episodes, each following a period of hydraulic stimulation. Following the first two episodes, a diverter was injected immediately after tracer injection.

\subsubsection{First Tracer Injection}

On 11/24/2012, $120 \mathrm{~kg}$ of $\mathrm{LiCl}$ and $25 \mathrm{~kg}$ of the disodium salt of 1,5-naphthalene disulfonic acid were added to 560 gal of water. The solution was recirculated to insure complete dissolution of the tracer salts. At 10:30 p.m., injection of the solution was initiated into the intake of the booster pump and maintained at a rate of $1.3 \mathrm{gpm}$ for the next $7 \mathrm{hr}$. The flow rate through the booster pump and into Well 55-29 was approximately 78 gpm. By 5:30 a.m. on 11/25/2012, all 560 gal of tracer solution had been pumped. Several wellbore volumes of water were then used to flush the well before the pumping of the first diverter pill.

\subsubsection{Second Tracer Injection}

On 12/3/2012, $25 \mathrm{~kg}$ of the disodium salt of 1,6-naphthalene disulfonic acid was added to approximately 1600 gal of water, which was recirculated to ensure complete mixing. The solution was then injected into Well 55-29 between 2:45 p.m. and 3:30 p.m. at a rate of approximately 35 gpm. After flushing one wellbore of freshwater, the second diverter pill was pumped. 


\subsubsection{Third Tracer Injection}

After the third stimulation episode, a third tracer injection was conducted. On 12/7/2012, $40 \mathrm{~kg}$ of $\mathrm{CsBr}$ and $25 \mathrm{~kg}$ of the disodium salt of 2,6-naphthalene disulfonic acid were added to 500 gal of water. The solution was recirculated to ensure complete dissolution of the tracer salts. At 3:50 p.m., the tracer solution was pumped into the injection line at a rate of approximately $12 \mathrm{gpm}$. By 4:30 p.m., the tank had emptied and was immediately flushed with an additional 500 gal of freshwater. During the tracer injection, the total flow rate into Well 55-29 was approximately $230 \mathrm{gpm}$. 


\subsection{Conclusion}

The primary goal of this project is to provide integrated tracer and tracer test interpretation tools to facilitate the interrogation of fracture apertures, fracture spacing, and temperature distributions in geothermal reservoirs through the use of multiple tracers that have different diffusion, adsorption, and thermal degradation properties. A secondary goal is to evaluate different tracer test designs and desirable tracer properties for interrogating these reservoir properties. The project was initially focused on the adaptation of PFT compounds to geothermal systems, and in particular on the evaluation of encapsulated PFTs that would release tracers at targeted reservoir temperatures (see Reimus et al. 2011). PFTs are non-reactive tracers used in numerous applications from atmospheric transport to underground leak detection, and they are attractive as tracers because of their extremely low detection limits and typically very low background concentrations. After the 2011 midyear DOE GTP review and subsequent discussions with the DOE GTP, emphasis was shifted to interpretive tool development, testing, and validation. Subsurface modeling capabilities are an important component of this project for both the design of suitable tracers and the interpretation of data from in situ tracer tests, whether they are single- or multi-well tests.

The capabilities of several software tools for modeling and interpreting tracer tests in EGS systems were introduced and demonstrated throughout this report. These tools include STOMP, TOUGHREACT, RELAP, and MULTRAN. Other than RELAP, all the models are numerical codes, and they have comparable capabilities, although each has its own strengths and limitations. STOMP and TOUGHREACT are fully 3D numerical models that can incorporate 3D reservoir features and spatial heterogeneity to the extent that these characteristics are known. MULTRAN is a 2D model that emphasizes the representation of diffusion and reaction processes and their temperature dependence in dual-porosity systems (thermal and mass diffusion, multicomponent mass transport that includes adsorption and cation exchange) while de-emphasizing the multidimensional representation of reservoir flow characteristics and spatial heterogeneity. The RELAP code is semi-analytical and is limited to simulating heat and mass transfer in interwell systems. However, RELAP's rapid execution makes it ideal for quick evaluations of tracer test designs and for inverse modeling of tracer tests if test conditions conform to the assumptions of the model. We do not make any explicit recommendations for tool usage, because such recommendations will depend on a variety of factors, including the available characterization data for an EGS system, the specifics of how a tracer test was conducted, and user familiarity with the different models.

Several important EGS tracer testing concepts were demonstrated during the project as documented in this report. These include the following:

- Thermally degrading tracers that do no adsorb to fracture or matrix surfaces can be used in conjunction with conservative tracers in interwell tracer tests to provide estimates of when thermal breakthrough will occur at a production well long before it actually occurs (Section 4.2). A semi-empirical method involving repeat injections of paired conservative and thermally degrading tracers was demonstrated and is believed to be ready for use by practitioners. The method was shown to be relatively robust for linear or biradial flow conditions, and for spatially variable fracture apertures and fracture spacings.

- The use of conservative tracers with different diffusion coefficients was shown to be a viable approach to estimating fracture apertures (or surface area to volume ratios) in both interwell and single-well tracer tests in EGS systems (Sections 4.3 and 5.3). This method requires a finite (nonzero) matrix 
porosity, and it works best when there are significant contrasts in the tracer matrix diffusion coefficients. The use of particle tracers with very small diffusion coefficients relative to solute tracers would significantly enhance the potential to use this method for interrogating fracture apertures. The method will be hindered by significant heterogeneity in matrix porosities and also by fracture-coating minerals that have significantly different porosities from the bulk matrix.

- The use of reactive tracers in conjunction with conservative tracers was shown to be a viable approach to estimating fracture apertures (or SA/V ratios) in both interwell and single-well tracer tests in EGS systems (Sections 4.4 and 5.3). However, the method is more robust in interwell tracer tests than single-well tracer tests, particularly if the matrix porosity is relatively small. In interwell tests the delayed arrival time of a reactive tracer relative to a conservative tracer serves as a more precise and conclusive interrogator of fracture apertures than the lower peak concentration and longer tails of reactive tracers in single-well tests.

- Cation-exchanging tracers can be used as a special case of reactive tracers in both interwell and singlewell tracer tests to estimate fracture apertures (or SA/V ratios) in EGS systems (Section 5.4), although they are clearly more practical in single-well tests because of their relatively high detection limits and typical background concentrations in geothermal reservoirs.

- A method was suggested for using temperature as a tracer in single-well injection-withdrawal tests to interrogate fracture spacings (Section 5.2). The method involves repeating the injection and withdrawal of cold water into and from a well using different injection times in each repeat test. The method may provide only a lower bound that fracture spacings must exceed, but this may still be useful information to the practitioner.

- The estimation of fracture surface area using a linear, reversibly adsorbing tracer in conjunction with a conservative tracer was demonstrated by interpreting an interwell tracer test at the Soda Lake, Nevada geothermal reservoir that was conducted by the EGI at the University of Utah in collaboration with Magma Energy, Inc. (Section 6.1). A detailed assessment of the uncertainties associated with the surface area estimates and recommendations for improving these estimates are provided. The TOUGHREACT model was used to predict single-well tracer test breakthrough curves at Soda Lake using the same conservative and adsorbing tracers that were used in the interwell tracer test.

- Two cation-exchanging tracers $\left(\mathrm{Li}^{+}\right.$and $\left.\mathrm{Cs}^{+}\right)$were deployed during fracture-zone stimulations at the Newberry Crater EGS demonstration site in late 2012 (Section 6.2). Several laboratory experiments were conducted to characterize the cation-exchange parameters in this system (Section 6.2.1) so that better-constrained estimates of fracture surface area could be obtained from the tracer return curves (which were still pending at the time of this report). The interpretation of the laboratory experiments exercised and demonstrated some of the capabilities of the MULTRAN (Section 6.2.1) and TOUGHREACT (Section 6.2.2) models, and they also revealed the important result that the cation exchange of $\mathrm{Li}^{+}$is highly temperature dependent, presumably because of the temperature dependence of its hydration characteristics. Pairings of cation-exchanging and conservative tracers were used to interrogate fracture surface area in three separate stimulation zones. Flowback from these tests was delayed until the spring of 2013 because of inclement weather conditions; therefore, the final results (i.e., analysis of tracer breakthrough curves) from these tracer tests were not available for inclusion in this report. 


\subsection{References}

Adams MC and J Davis. 1991. "Kinetics of Fluorescein Decay and its Application as a Geothermal Tracer." Geothermics 20:53-66.

Andersson P, J Byegard, W Dershowitz, T Doe, J Hermanson, P Meier, E Tullborg, and A Winberg. 2002. TRUE Block Scale Project. Final Report 1(4). Characterisation and Model Development. Technical Report 02-13, Swed. Nucl. Fuel and Waste Manage. Co., Stockholm, Sweden.

AltaRock Energy. 2011. Newberry Enhanced Geothermal Systems Demonstration: Phase I Report. AltaRock Energy Inc., Seattle, Washington.

Appelo CAJ. 1994a. "Cation and Proton Exchange, $\mathrm{pH}$ Variations, and Carbonate Reactions in a Freshening Aquifer." Water Resources Research 30(10):2793-2805.

Appelo CAJ. 1994b. "Some Calculations on Multicomponent Transport with Cation Exchange in Aquifers." Groundwater 32(6):968-975.

Appelo CAJ. 1996. "Multicomponent ion exchange and chromatography in natural systems." In: Reactive Transport in Porous Media. PC Lichtner, CI Steefel, and EH Oelkers (eds.). Reviews in Mineralology 34:193-227.

Bowman RS. 1984. "Evaluation of some new tracers for soil water studies." Soil Science Society of America Journal 48 (5):987-993.

Bruseau ML, KM Bronson, S Ross, NT Nelson, and TD Carlson. 2003. “Application of Gas-Phase Partitioning Tracer Test to Characterize Immiscible-Liquid Contamination in the Vadose Zone Beneath a Fuel Depot." Vadose Zone Journal 2:148-153.

Chemburkar RM, LF Brown, BJ Travis, and BA Robinson. 1991. "Numerical Determination of Temperature Profiles in Flowing Systems from Conversions of Chemically Reacting Tracers." Chemical Engineering Science 46(5):1349-1360.

Cladouhos TT, S Petty, Y Nordin, M Moore, K Grasso, M Uddenberg, M Swyer, B Julian, and G Foulger. 2013. "Microseismic Monitoring of Newberry Volcano EGS Demonstration." In: Proceedings of the Thirty-Eighth Workshop on Geothermal Reservoir Engineering, Stanford University, Stanford, California, February 11-13, 2013.

Cvetkovic V. 2010. "Significance of fracture rim zone heterogeneity for tracer transport in crystalline rock." Water Resources Res. 46, W03504, doi:10.1029/2009WR007755.

Dean C, P Reimus, K Leecaster, J Oates, D Newell, and S Petty. 2013. "Laboratory Experiments to Characterize Cation-Exchanging Tracer Behavior at Newberry Crater, OR." In: Proceedings of the Thirty-Eighth Workshop on Geothermal Reservoir Engineering, Stanford University, Stanford, California, February 11-13, 2013. 
Dean CA, P Reimus, and D Newell. 2012. "Evaluation of a Cation Exchanging Tracer to Interrogate Fracture Surface Area in EGS Systems." In: Proceedings of the Thirty-Seventh Workshop on Geothermal Reservoir Engineering, Stanford University, Stanford, California, January 30-February 1, 2012.

Deng H, Z Dai, A Wolfsberg, Z Lu, M Ye, and P Reimus. 2010. "Upscaling of reactive mass transport in fractured rocks with multimodal reactive mineral facies." Water Resources Res. 46, W06501, doi:10.1029/2009WR008363.

Echols J, D Benoit, M Ohren, G Oppliger, and T Van Gundy. 2011. "Integration of a 3D-3C Reflection Seismic Survey over a Known Geothermal Resource: Soda Lake, Churchill County, Nevada.” GRC Transactions Vol. 35.

Farnham IM, LC Meigs, ME Dominguez, K Lindley, JM Daniels, and KJ Stetzenbach. 1997. "Evaluation of tracers used for the WIPP tracer tests," Appendix H in: Meigs, LC, RL Beauheim, TL Jones (Eds.), Interpretations of Tracer Tests Performed in the Culebra Dolomite at the Waste Isolation Pilot Plant Site. SAND97-3109, Sandia National Laboratories, Albuquerque, New Mexico.

Fayer S, P Rose, S Petty, MD Deo, and T Xu. 2009. "A Computational Technique for Estimating the Fracture Surface Area Adjacent to a Newly Stimulated Well within an Engineered Geothermal System." In: Proceedings of TOUGH Symposium 2009, Lawrence Berkeley National Laboratory, Berkeley, California, September 14-16, 2009.

Gaines GL and HC Henry. 1955. "Adsorption Studies on Clay Minerals. V. Montmorillonite-CesiumStrontium at Several Temperatures." The Journal of Chemical Physics 23:2322.

Gast RG and WD Klobe. 1971. "Sodium-lithium exchange equilibria on vermiculite at 25 degrees and 50 degrees C." Clays and Clay Minerals 19(5):311-319.

Kibbey TCG, R Paruchuri, DA Sabatini, and L Chen. 2007. "Adsorption of beta blockers to environmental surfaces.” Environ. Sci. Technol. 41:5349-5356.

Kraus KA and RJ Raridon. 1959. "Temperature Dependence of some Cation Exchange Equilibria in the Range 0 to $200^{\circ}$." The Journal of Physical Chemistry 63(11):1901-1907.

Leecaster K, B Ayling, G Moffitt, S Clausen, and PE Rose. 2012. "Use of Safranin T as a Reactive Tracer for Geothermal Reservoir Characterization." In: Proceedings of the Thirty-Seventh Workshop on Geothermal Reservoir Engineering, Stanford University, Stanford, California, January 30-February 1, 2012.

Likhachev ER. 2003. "Dependence of water viscosity on temperature and pressure." Technical Physics, 48(4):514-515 (translated from Zhurnal Tekhnichoskoi Fiziki, 73(4):135-136, 2003).

Maloszewski P and A Zuber. 1984. "Interpretation of artificial and environmental tracers in fissured rocks with a porous matrix." In: Isotope Hydrology, pp. 635-651, International Atomic Energy Agency, Vienna, Austria. 
Maloszewski P and A Zuber. 1985. "On the theory of tracer experiments in fissured rocks with a porous matrix." Journal of Hydrology 79:333-358.

MIT (Massachusetts Institute of Technology). 2006. The Future of Geothermal Energy, Impact of Enhanced Geothermal Systems (EGS) on the United States in the 21st Century. Cambridge, Massachusetts.

Newman JS. 1973. Electrochemical Systems. Prentice-Hall, Englewood Cliffs, New Jersey.

Nicol DAC and BA Robinson. 1990. "Modelling the Heat Extraction from the Rosemanowes HDR Reservoir." Geothermics 19(3):247-257.

O'Sullivan MJ, K Pruess, and MJ Lippman. 2001. "State of the art of geothermal reservoir simulation." Geothermics 30:395-429.

Petty S, Y Nordin, W Glassley, TT Cladouhos, and M Swyer. 2013. Improving Geothermal Project Economics with Multi-Zone Stimulation: Results from the Newberry Volcano EGS Demonstration. Proceedings, Thirty-Eighth Workshop on Geothermal Reservoir Engineering, Stanford University, Stanford, California, February 11-13, 2013.

Pruess K. 1983. GMINC-A Mesh Generator for Flow Simulations in Fractured Reservoirs. LBL-15227, Lawrence Berkeley Laboratory, Berkeley, California.

Pruess K and TN Narasimhan. 1982. A Practical Method Fluid and Heat Flow in Fractured Porous Media. LBL-13487, Lawrence Berkeley Laboratory, Berkeley, California.

Pruess K. 1992. Brief Guide to the MINC-Method for Modeling Flow and Transport in Fractured Media. LBL-32195, Lawrence Berkeley Laboratory, Berkeley, California.

Pruess K, C Oldenburg, and G Moridis. 1999. TOUGH2 user's guide. Version 2.0. LBL-43134, Lawrence Berkeley Laboratory, Berkeley, California.

Pruess K and C Doughty. 2010. “Thermal Single-Well Injection-Withdrawal Tracer Tests for Determining Fracture-Matrix Heat Transfer Area." In: Proceedings: Thirty-Fifth Workshop on Geothermal Reservoir Engineering, Stanford University, Stanford, California, February 1-3, 2010.

Reimus PW, A Jackson, and O Karazincir. 2012. "Interrogating Surface Area in Oil Shales using GasPhase Tracers: Status as of June 2010." Interim Report for CRADA with Chevron Energy Technology Company, Los Alamos National Laboratory, Los Alamos, New Mexico (currently not available to public, but included gas adsorption surface area measurements on F-95 Ottawa sand).

Reimus P. 2010. "A Semi-Analytical Model for Heat and Mass Transfer in Geothermal Reservoirs to Estimate Fracture Surface-Area-to-Volume Ratios and Thermal Breakthrough using Thermally-Decaying and Diffusing Tracers." Abstract H23I-03 presented at 2010 Fall Meeting, American Geophysical Union, San Francisco, California., 13-17 December 2010. 
Reimus PW and TJ Callahan. 2007. "Matrix Diffusion Rates in Fractured Volcanic Rocks at the Nevada Test Site: Evidence for a Dominant Influence of Effective Fracture Apertures." Water Resources Research 43, W07421, doi:10.1029/2006WR005746.

Reimus PW, TJ Callahan, SD Ware, MJ Haga, and DA Counce. 2007. "Matrix diffusion coefficients in volcanic rocks at the Nevada test site: Influence of matrix porosity, matrix permeability, and fracture coating minerals." J. Contaminant Hydrol, 93:87-95.

Reimus PW, G Pohll, T Mihevc, J Chapman, L Papelis, B Lyles, S Kosinski, R Niswonger, and P Sanders. 2003. "Testing and Parameterizing a Conceptual Model for Radionuclide Transport in a Fractured Granite using Multiple Tracers in a Forced-Gradient Test.” Water Resources Research 39(12):1350, doi:10.1029/2002WR001597.

Reimus P, T Watson, V Vermeul, D Newell, and M Williams. 2011. "Laboratory Testing and Modeling to Evaluate Perfluorocarbon Compounds as Tracers in Geothermal Systems." In: Proceedings of the Thirty-Sixth Workshop on Geothermal Reservoir Engineering, Stanford University, Stanford, California, January 31-February 2, 2011.

Reimus P, M Williams, V Vermeul, P Rose, K Leecaster, B Ayling, R Sanjuan, M Ames, C Dean, and D Benoit. 2012. "Use of Tracers to Interrogate Fracture Surface Area in Single-Well Tracer Tests in EGS Systems." In: Proceedings of the Thirty-Seventh Workshop on Geothermal Reservoir Engineering, Stanford University, Stanford, California, January 30-February 1, 2012.

Robinson BA, JW Tester, and LF Brown. 1988. "Reservoir Sizing Using Inert and Chemically Reacting Tracers." SPE Formation Evaluation 3:227-234.

Rose PE and MC Adams. 1994. "The Application of Rhodamine WT as a Geothermal Tracer." Geothermal Resources Council Transactions 18:237-240.

Rose P, K Leecaster, S Clausen, R Sanjuan, M Ames, P Reimus, M Williams, V Vermeul, and D Benoit. 2012. "A Tracer Test at the Soda Lake, Nevada Geothermal Field Using a Sorbing Tracer." In:

Proceedings of the Thirty-Seventh Workshop on Geothermal Reservoir Engineering, Stanford University, Stanford, California, January 30-February 1, 2012.

Rose P, J Harris, M Mella, S Clausen, J Oates, P Reimus, C Dean, M Williams, V Vermeul, B Boyes, and S Petty. 2013. Tracer Methods for Characterizing Fracture Creation in Engineered Geothermal Systems: Progress Report for Quarter Ending March 31, 2013. DOE GTP Project GO18193, Energy and Geosciences Institute, Salt Lake City, Utah.

Sandia National Laboratories. 2007. "Site-Scale Saturated Zone Transport, Appendix A: Technical Basis for Sorption Coefficient Probability Distribution Functions." MDL-NBS-HS-000010, Rev 03, prepared for the U.S. Department of Energy Office of Civilian Radioactive Waste Management, Albuquerque, New Mexico.

Sennum GI, RN Dietz, TW D'Otavvio, RW Goodrich, EA Cote, and DJ Spandau. 1989. A Perfluorocarbon Tracer Transport and Dispersion Experiment in the North Sea Ekofisk Oil Field. BNL-43811, Brookhaven National Laboratory, Upton, New York. 
Sullivan EJ, PW Reimus, and DA Counce. 2003. "Transport of a Reactive Tracer in Saturated Alluvium Described using a Three-Component Cation-Exchange Model." Journal of Contaminant Hydrology 62-63:675-694.

Tang JS. 1995. "Partitioning Tracers and In-Situ Fluid Saturation Measurements." SPE Formation Evaluation. Society of Petroleum Engineers, 10(1) pp. 33-39.

Triay IR, A Meijer, JL Conca, KS Kung, RS Rundberg, BA Strietelmeier, CD Tait, DL Clark, MP Neu, and DE Hobart. 1997. Summary and Synthesis Report on Radionuclide Retardation for the Yucca Mountain Site Characterization Project. Yucca Mountain Site Characterization Program Milestone 3784M, LA-13262-MS, Los Alamos National Laboratory, Los Alamos, New Mexico.

Tsang YW. 1992. "Usage of 'equivalent apertures' for rock fractures as derived from hydraulic and tracer tests." Water Resources Res. 28(5):1451-1455.

Warren JE and PJ Root. 1963. "The Behavior of Naturally Fractured Reservoirs." Society of Petroleum Engineers Journal. Transactions of the American Institute of Mining Engineers 228:245-255.

Watson TB, R Wilke, RN Dietz, J Heiser, and P Kalb. 2007. "The Atmospheric Background of Perfluorocarbon Compounds Used as Tracers.” Environmental Science and Technology 41:6909-6913.

White MD and M Oostrom. 2000. STOMP Subsurface Transport Over Multiple Phase: Theory Guide. PNNL-11216 (UC-2010), Pacific Northwest National Laboratory, Richland, Washington.

White MD and M Oostrom. 2006. STOMP Subsurface Transport Over Multiple Phase: User's Guide. PNNL-15782 (UC-2010), Pacific Northwest National Laboratory, Richland, Washington.

Winberg A et al. 2002. Final Report of the TRUE Block Scale Project. Part 4. Synthesis of Flow, Transport and Retention in the Block Scale. Tech. Rep. 02-16, Swed. Nucl. Fuel and Waste Manage. Co., Stockholm, Sweden.

Xu T, E Sonnenthal, N Spycher, and K Pruess. 2006. TOUGHREACT V1.2 User's Guide: A Simulation Program for Nonisothermal Multiphase Reactive Geochemical Transport in Variably Saturated Geologic Media. LBNL-55460, Lawrence Berkeley Laboratory, Berkeley, California.

Xu T, N Spycher, E Sonnenthal, L Zheng, and K Pruess. 2012. TOUGHREACT User's Guide: A Simulation Program for Non-isothermal Multiphase Reactive Transport in Variably Saturated Geologic Media, Version 2.0. LBNL June 2012 Draft, Lawrence Berkeley Laboratory, Berkeley, California. 


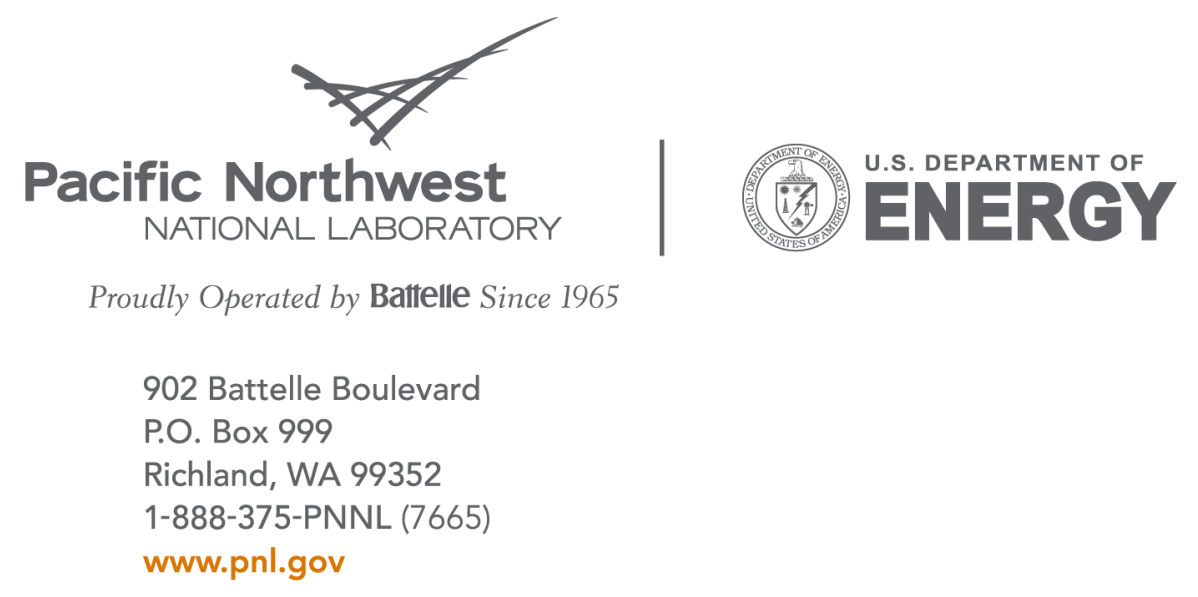

